\title{
Autonomous UAV Control for Low-Altitude Flight in an Urban Gust Environment
}

by

\author{
Syed Ali Raza \\ A dissertation submitted to \\ the Faculty of Graduate Studies and Research \\ in partial fulfilment of \\ the requirements for the degree of \\ Doctor of Philosophy
}

\author{
Ottawa-Carleton Institute for \\ Mechanical and Aerospace Engineering \\ Department of Mechanical and Aerospace Engineering \\ Carleton University \\ Ottawa, Ontario, Canada
}

August 2015

Copyright (C)

2015 - Syed Ali Raza 
The undersigned recommend to

the Faculty of Graduate Studies and Research

acceptance of the dissertation

\title{
Autonomous UAV Control for Low-Altitude Flight in an Urban Gust Environment
}

\author{
Submitted by Syed Ali Raza \\ in partial fulfilment of the requirements for the degree of \\ Doctor of Philosophy
}


Dr. Jason Etele, Supervisor

Dr. Hugh H.T. Liu, External Examiner

Dr. Jeff Dawson, Internal Examiner

Dr. Robert G. Langlois, Member of Department

Dr. Steve Ulrich, Member of Department

Dr. Davide Spinello, Member of Joint Institute

Metin Yaras, Department Chair

Carleton University

2015

iii 


\section{Abstract}

With rapid advances in the unmanned aerial vehicle (UAV) field and their growing popularity in a wide range of civilian and commercial applications, UAV operation in urban areas is inevitable. For small-size UAVs conducting low-level flight in an urban landscape, wind disturbances pose a significant challenge. Ensuring safety while flying in proximity to buildings and other obstacles requires a thorough understanding of the nature of these disturbances and the expected performance of an autopilot in their presence. This study focuses on the position control of a quadrotor UAV in an urban wind environment. A literature review provides an in-depth survey of the state of the art in quadrotor flight control. Urban wind conditions are modelled around a single building through a Computational Fluid Dynamics (CFD) analysis using Large Eddy Simulation (LES). Modelled transient wind flow velocities are applied to create a realistic simulation environment for a custom-built quadrotor prototype named TARA. Four different control techniques are selected and implemented for the autonomous position control of TARA. A precise simulation methodology is employed to ensure consistent flight testing under diverse representative wind conditions. The results are evaluated under a carefully-crafted set of criteria and selected performance metrics. Based on the analysis, a hybrid control scheme is proposed, with simulation and experimental data confirming its improved ability in dealing with realistic urban wind disturbances with an average position hold within a single body length. 


\section{Acknowledgments}

And then it was over. With congratulations from professors and colleagues, this doctoral research spanning over the past five years and eight months had successfully come to an end. Looking back there are many important individuals who played crucial roles in helping me reach the finish line. I will begin with a sincere thanks to Professor Joshua Marshall who most graciously recommended me to Professor Jason Etele.

During my time working under the stellar supervision and mentorship of Professor Etele I have enjoyed a research environment with the freedom to explore and try new ideas. I would like to convey my utmost gratitude for your complete support and understanding throughout this endeavour. Your impressive ability to deduce control actions from flight test videos frame by frame taught me to pay attention to minute details in order to obtain meaningful results. Over many iterations you have helped me refine this thesis into its present state. A special thanks for bringing together our team of graduate students over memorable breakfast meetings, and feedback-rich progress report presentations, which helped me in improving my work over these years.

Many thanks as well to Dr. Giovanni Fusina of DRDC for funding this research without which it would have been impossible for me to complete this work.

I would like to acknowledge the MAE laboratory staff members, beginning with 
Steve Truttmann for his friendship and overall helpfulness in conducting my laboratory teaching sessions. Stephan Biljan for helping in rotorcraft laboratory experiments and the 3D printing of replacement parts for TARA. I would also like to thank Alex Proctor and Kevin Sangster for their guidance in the machine shop, with special thanks to Alex who always helped me unlock the flight mechanics lab.

My friend and colleague Aliaksandr Murzionak helped in the initial CAD design and 3D printing of TARA. I am grateful for all of your assistance in flight testing and your courageous offer be the launch pad for the maiden flight of TARA. My best wishes for you in completing your doctoral research with success.

Another friend and colleague Mark Sutherland was always present and always willing to help. Your research provided important data for this project. I would like to especially thank you for all of your help in the outdoor flight testing of TARA. May you be successful and happy in your future endeavours.

I would like to thank my office mate Hooman Hanachi for many interesting and insightful conversations, as well as sharing your expertise.

On a personal level, my parents have provided me with their unconditional support in every step of my life, and always encouraged me to aim for the best.

Final thanks goes to the person holding multiple titles of a friend, partner, editorin-chief, mommy, and wife, Shaista whose help was instrumental at every step for the past many years. Your strong support allowed me to complete this thesis and made these student years a pleasant and memorable adventure.

This has been a long journey, with many ups and downs. In 2011, I lost my mother Tasneem Ara. In 2012, I welcomed my twin sons Haidar and Abbas, who are the joy of my life. This thesis is dedicated to the three of them. 


\section{Table of Contents}

Abstract $\quad$ iv

Acknowledgments $\quad$ V

Table of Contents vii

List of Tables $\quad$ xii

List of Figures $\quad$ xiv

$\begin{array}{ll}\text { Acronyms } & \text { xviii }\end{array}$

Nomenclature $\quad$ xxi

1 Introduction 1

1.1 Motivation . . . . . . . . . . . . . . . . . . 1

1.2 Problem Statement . . . . . . . . . . . . . . . . . 3

1.3 Organization of Thesis . . . . . . . . . . . . . 5

2 Literature Review $\quad 6$

2.1 Autonomous Flight Control of Quadrotors . . . . . . . . . . . . 6

2.2 PID Control . . . . . . . . . . . . . . . . . 7

2.3 Lyapunov-based Methods . . . . . . . . . . . . . . . . . . 12

2.4 Optimal Control . . . . . . . . . . . . . . . . . . . . . . . 18 
2.5 Robust Control . . . . . . . . . . . . . . . . . . . . . 20

2.6 Adaptive Control . . . . . . . . . . . . . . . . . . . 23

2.7 Intelligent Control . . . . . . . . . . . . . . . . 25

2.8 UAV Flight Performance in Wind Gust Conditions . . . . . . . . . . 31

2.9 Summary . . . . . . . . . . . . . . . . . . . 37

3 Simulation Methodology $\quad 40$

3.1 Flight Simulation with Urban Wind Model . . . . . . . . . . . . . 41

3.1.1 Mathematical Modeling of TARA . . . . . . . . . . . . . 41

3.1.2 Equations of Motion . . . . . . . . . . . . . . 47

3.1.3 Experimental Identification of Parameters . . . . . . . . . . . 48

3.1.4 TARA Flight Simulator . . . . . . . . . . . . . 50

3.2 Large Eddy Simulation Based Wind Model . . . . . . . . . . . . . 54

3.3 Single Building Test Points . . . . . . . . . . . . . . . . . 62

3.3.1 Dead Air Zone (Point-A) . . . . . . . . . . . . . . 64

3.3.2 Passing Vortex (Point-B) . . . . . . . . . . . . 64

3.3.3 Wake Eddy (Point-C) . . . . . . . . . . . . 68

3.3.4 Downwash (Point-D) . . . . . . . . . . . . 69

3.3.5 Accelerated Freestream (Point-E) . . . . . . . . . . . . 69

3.3.6 Freestream Wind (Point-F) . . . . . . . . . . 73

3.4 Criteria for Evaluating and Comparing Control Performances . . . . . 75

4 Control Methodologies $\quad 79$

4.1 PID control . . . . . . . . . . . . . . . . . . 80

4.1.1 Attitude Stabilization . . . . . . . . . . . . . . . 80

4.1.2 Attitude Stabilization Results . . . . . . . . . . . . . 82

4.1.3 PID Position Control . . . . . . . . . . . . . . . 85

4.2 Integral Backstepping Position Control . . . . . . . . . . . . . 87 
4.2.1 Lyapunov Stability Theory . . . . . . . . . . . . . . . . . . 88

4.2.2 Backstepping Control Technique . . . . . . . . . . . . . . . 90

4.2.3 Integral Backstepping Control Law Synthesis . . . . . . . . . . 93

4.2.4 Simplified Model for Control Design . . . . . . . . . . . . . . . 94

4.2.5 Integral Backstepping Altitude Control . . . . . . . . . . . . . 95

4.2.6 Stability Analysis of Altitude Control Law . . . . . . . . . . . 98

4.2.7 Integral Backstepping Lateral Position Control . . . . . . . . . 98

4.3 Fuzzy Control . . . . . . . . . . . . . . . . . . . . . 102

4.3.1 The Fuzzy Control Law . . . . . . . . . . . . . . . . . 103

4.3.2 Fuzzy Altitude Control . . . . . . . . . . . . . . . . . 110

4.3.3 Fuzzy Lateral Position Control . . . . . . . . . . . . . . . . . 111

4.4 Adaptive Control . . . . . . . . . . . . . . . . . . . . . . . . 112

4.4.1 Adaptive Integral Backstepping Altitude Control . . . . . . . 113

4.4.2 Stability Proof of AIB Altitude Control Law . . . . . . . . . . 116

4.4.3 Adaptive Integral Backstepping Lateral Position Control . . . 117

4.5 Step Response Performance Evaluation . . . . . . . . . . . . . . . . . 118

4.5.1 X-axis Step Response . . . . . . . . . . . . . . . . . 119

4.5.2 Y-axis Step Response . . . . . . . . . . . . . . . . . . 121

4.5.3 Z-axis Step Response . . . . . . . . . . . . . . . . . . . 121

5 Results $\quad 126$

5.1 Position Control Simulations in Wind . . . . . . . . . . . . . . 127

5.1.1 Dead Air Zone (Point-A) . . . . . . . . . . . . . 127

5.1 .2 Passing Vortex (Point-B) . . . . . . . . . . . . 128

5.1.3 Wake Eddy (Point-C) . . . . . . . . . . . . . 130

5.1 .4 Downwash $($ Point-D) . . . . . . . . . . . . . . . 132

5.1.5 Accelerated Freestream (Point-E) . . . . . . . . . . . 134 
5.1.6 Freestream Wind (Point-F) _. . . . . . . . . . . 136

5.2 Review . . . . . . . . . . . . . . . . . . . . . . . 138

5.3 Experimental Flight Results . . . . . . . . . . . . . . . . . 140

5.3.1 PD/PID based Indoor Position Control (No Wind) . . . . . 140

5.3.2 PD/PID-based Outdoor Position Control . . . . . . . . . . . 143

6 Hybrid Control 148

6.1 Proposed Hybrid Control Scheme . . . . . . . . . . . . . . . . 148

6.2 Hybrid Position Control Simulations . . . . . . . . . . . . . 153

6.2.1 Dead Air Zone (Point-A) . . . . . . . . . . . . . . . . 154

6.2.2 Passing Vortex (Point-B) . . . . . . . . . . . . . . . 154

6.2 .3 Wake Eddy $($ Point-C) . . . . . . . . . . . . . . . . . 157

6.2.4 Downwash (Point-D) . . . . . . . . . . . . . . 157

6.2.5 Accelerated Freestream (Point-E) _ . . . . . . . . . 158

6.2.6 Freestream Wind (Point-F) f . . . . . . . . . . 160

6.3 Summary of Hybrid Position Control Simulations . . . . . . . . . 161

6.4 Experimental Outdoor Flight using Hybrid Position Control . . . . . 162

$\begin{array}{lll}7 \text { Conclusion } & 167\end{array}$

7.1 Summary of Contributions . . . . . . . . . . . . . . . . . . . 169

7.2 Future Research . . . . . . . . . . . . . . . . . . . 170

List of References $\quad 172$

$\begin{array}{lll}\text { Appendix A Design and Development of TARA } & 187\end{array}$

A.1 Airframe Design . . . . . . . . . . . . . . . . . . . . . . . 189

A.2 Propulsion System . . . . . . . . . . . . . . . . 190

A.3 Power Supply . . . . . . . . . . . . . . . . . . . . . . . . 193

A.4 Orientation Sensors . . . . . . . . . . . . . . . . 193 
A.5 Positioning Sensors . . . . . . . . . . . . . . . . . . 195

A.6 Onboard Processors . . . . . . . . . . . . . . . . . . . . . . . . 199

A.7 Communication Hardware . . . . . . . . . . . . . 200

A.8 Software Implementation . . . . . . . . . . . . . . . . . 201

Appendix B Sonar Based Position Estimation 203

B.1 Range Measurement and Position Estimation . . . . . . . . . . 204

B.2 Heading Independent Lateral Position Control Scheme . . . . . . 208

$\begin{array}{ll}\text { Appendix C Position Control Simulations } & 211\end{array}$

C.1 Position Control Simulations without Wind . . . . . . . . . 211

C.1.1 Sample Position Hold at Point-B . . . . . . . . . . . . . 212

C.2 In wind Position Control Simulation Plots . . . . . . . . . . . . 212

C.3 Experimental Outdoor Flight Plots . . . . . . . . . . . . . . 212 


\section{List of Tables}

1 Commercial off-the-shelf quadrotor specifications . . . . . . . . . . . . 2

2 Position control performance metric . . . . . . . . . . . . 78

3 Finalized attitude controller gains . . . . . . . . . . . . . 82

4 Attitude stabilization performance metric . . . . . . . . . . . . . . 82

$5 \quad$ PID position controller gains . . . . . . . . . . . . . . . 87

6 Gains for the IB position controllers . . . . . . . . . . . . . . . . . . . 102

7 The fuzzy rule base . . . . . . . . . . . . . . . . . 107

8 Gains for the AIB position controllers . . . . . . . . . . . . . 118

$9 \quad$ X-axis step response summary . . . . . . . . . . . . . . . . 121

$10 \quad$ Y-axis step response summary . . . . . . . . . . . . . . . . . . 123

11 Z-axis step response summary . . . . . . . . . . . . . . . 125

12 In wind control performance summary of position hold at point-A . . 128

13 In wind control performance summary of position hold at Point-B . . 130

14 In wind control performance summary of position hold at point-C . . 132

15 In wind control performance summary of position hold at point-D . . 134

16 In wind control performance summary of position hold at point-E . . 136

17 In wind control performance summary of position hold at point-F . . 138

18 PID outdoor position control summary . . . . . . . . . . . . . 146

$19 \mathrm{PD} / \mathrm{PID}$ simulation position hold summary . . . . . . . . . . . 146

20 In wind hybrid control performance summary . . . . . . . . . . 153 
21 Hybrid outdoor position control summary . . . . . . . . . . . . 165

22 GPGGA fixed data message template . . . . . . . . . . . . . . . 197

23 GPRMC minimum specific GNSS data message template . . . . . . . 198

24 No wind control performance summary of position hold at point-B . . 212 


\section{List of Figures}

1 Earth and body reference frames . . . . . . . . . . . . 41

2 Thrust plotting of experimental data $\ldots \ldots \ldots \ldots$

3 Simulink model of TARA flight simulator . . . . . . . . . . . . 51

4 TARA UAV block . . . . . . . . . . . . . . . . . . 52

5 Propulsion model for a single motor . . . . . . . . . . . . . . 52

6 The control stage in the flight controller block . . . . . . . . . 54

7 The post-processing stage in the flight controller block $\ldots \ldots \ldots$

8 Flow past a circular cylinder with different flow regimes . . . . . . 56

9 Hydrogen bubble visualization of flow past a square cube . . . . . 57

10 Large Eddy Simulation for computing flow around a square body . . 58

11 Large Eddy Simulation based flow around a square body . . . . . . 58

12 The CFD domain side view for the large eddy simulations . . . . . 59

13 The CFD domain top view for the large eddy simulations . . . . . . 60

14 Test points A to $\mathrm{F}$ around the symmetry plane of a single building . . 63

15 Wind flow at point-A ................... 65

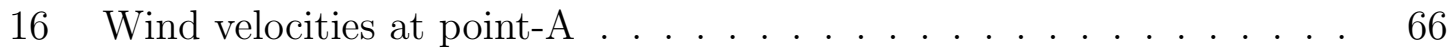

17 Wind flow at point-B . . . . . . . . . . . 67

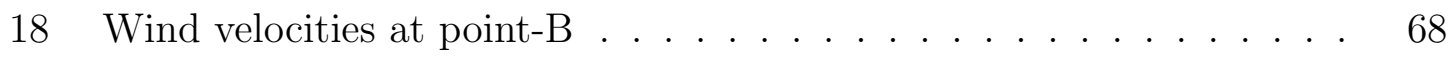

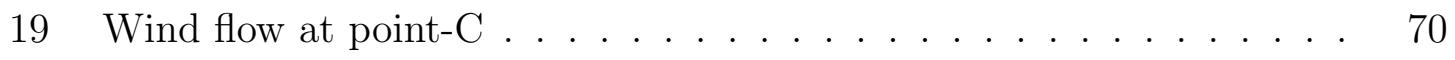

20 Wind velocities at point-C . . . . . . . . . . 71 
21 Wind flow at point-D . . . . . . . . . . . . . . . 72

22 Wind velocities at point-D . . . . . . . . . . . . . 73

23 Wind flow at point-E . . . . . . . . . . . . 74

24 Wind velocities at point-E . . . . . . . . . . . . . 75

25 Wind velocities at point-F . . . . . . . . . . . 76

26 Flight control system architecture . . . . . . . . . . . . . . 80

27 Pitch angle flight data . . . . . . . . . . . . . . . 83

28 Roll angle flight data . . . . . . . . . . . . . . . . . . 84

29 Yaw angle flight data . . . . . . . . . . . . . 86

30 The backstepping control . . . . . . . . . . . . . . . . 92

31 Membership functions for error input . . . . . . . . . . . . . . . 104

32 Membership functions for error rate input . . . . . . . . . . . 105

33 Membership functions for fuzzy output . . . . . . . . . . . . 106

34 Mamdani fuzzy inferencing method . . . . . . . . . . . . . . 108

35 Aggregation method and crisp output . . . . . . . . . . . . . 109

36 The surface plot of the fuzzy position controller . . . . . . . . . . 109

37 The fuzzy altitude controller . . . . . . . . . . . . . . . . . 110

38 The fuzzy X-axis position controller . . . . . . . . . . . . . . 111

39 X-axis step response f . . . . . . . . . . . . . . . . . . . 120

$40 \quad$ Y-axis step response . . . . . . . . . . . . . . . . 122

41 Z-axis step response . . . . . . . . . . . . . . . . . . 124

42 In wind position hold for dead air zone (point-A) . . . . . . . . . . . 129

43 In wind position hold for passing vortex (Point-B) . . . . . . . . . . . 131

44 In wind position hold for wake eddy (point-C) . . . . . . . . . 133

45 In wind position hold for downwash (point-D) . . . . . . . . 135

46 In wind position hold for accelerated freestream (point-E) . . . . . . 137

47 In wind position hold for freestream wind (point-F) . . . . . . . . 139 
48 Experimental indoor flight test setup . . . . . . . . . . . . . . . . . . 141

49 Indoor flight test: X-axis position error . . . . . . . . . . . . . . . . . 142

50 Indoor flight test: Y-axis position error . . . . . . . . . . . . . . . . . 142

51 Indoor flight test: Altitude control . . . . . . . . . . . . . . 143

52 Experimental outdoor flight test setup . . . . . . . . . . . . . . . . . 144

53 PID outdoor position control experiment . . . . . . . . . . . . . 147

54 Distribution of $\mathrm{X}$-axis wind at point-B . . . . . . . . . . . 149

55 X-axis control output for position hold at point-B . . . . . . . . . . . 150

56 Distribution of X-axis wind at point-E . . . . . . . . . . . 151

57 X-axis control output for position hold at point-E . . . . . . . . . 152

58 In wind hybrid position hold for point-A . . . . . . . . . . . 155

59 In wind hybrid position hold for point-B . . . . . . . . . . . . . 156

60 In wind hybrid position hold for point-C . . . . . . . . . . . . 158

61 In wind hybrid position hold for point-D . . . . . . . . . . . . 159

62 In wind hybrid position hold for point-E . . . . . . . . . . . . 160

63 In wind hybrid position hold for point-F . . . . . . . . . . . 161

64 PD/PID, AIB and hybrid controller error comparison . . . . . . . . 163

65 Hybrid outdoor position control experiment plots . . . . . . . . . . 166

66 TARA quadrotor UAV prototype. . . . . . . . . . . . . 187

67 TARA's system design . . . . . . . . . . . . . . . . . 188

68 The CAD model of TARA. . . . . . . . . . . . . . . . . . . . . . 189

69 The Rimfire 400, 28-30-950 BLDC motor. . . . . . . . . . . . . . . 190

70 The PWM timing diagram. . . . . . . . . . . . . . . . . 191

71 The DJI Series 30 A OPTO ESC. . . . . . . . . . . . . . . 192

72 The APC counter rotating propeller pair. . . . . . . . . . . . . . . 192

73 The LIS3L02AL triple axis accelerometer. . . . . . . . . . . . . 193

74 The IDG-600 based triple axis gyroscope. . . . . . . . . . . . . . . 194 
75 The HMC5883L digital compass module. . . . . . . . . . . . . . . 194

76 The MaxBotix MB1340 Sonar Sensor. . . . . . . . . . . . . . . . . . 196

77 The MN5010HS Micro-Mini GPS module. . . . . . . . . . . . . 196

78 The Axon ATMega640 based controller board . . . . . . . . . . . 199

79 The Arduino Pro Mini 328 board . . . . . . . . . . . . . . . . . . . 199

80 The Xbee Pro wireless transciever module . . . . . . . . . . 200

81 The sonar sensor module mounted at the end of an arm . . . . . . . 205

82 Daisy chaining sonar sensors . . . . . . . . . . . . . . . . 206

83 Simultaneous triggering sonar sensors . . . . . . . . . . . . 206

84 Thrust plotting of experimental data . . . . . . . . . 207

85 Updated position control scheme. . . . . . . . . . . . . . . . . . 209

86 No wind position hold for passing vortex (Point-B) . . . . . . . . 213

87 Full flight test plot for X-axis . . . . . . . . . . . . . . 214

88 Full flight test plot for Y-axis . . . . . . . . . . . . 215

89 Full flight test plot for Z-axis _ . . . . . . . . . . . . . . . 216

90 PID outdoor position control experiment plots . . . . . . . . . 217

91 Hybrid outdoor position control experiment plots . . . . . . . . . 218 


\section{Acronyms}

ABS Acrylonitrile Butadiene Styrene

ADC Analog to Digital Converter

AIB Adaptive Integral Backstepping

AOAD Average of Absolute Difference

BLDC Brushless Direct Current

CAD Computer Aided Design

CFD Computational Fluid Dynamics

CFRP Carbon Fiber Reinforced Polymer

CFTO Constrained Finite Time Optimal

COA Center of Area

COG Center of Gravity

CRI Compositional Rule of Inference

DOF Degree Of Freedom

EKF Extended Kalman Filter

EMI Electromagnetic Interference 
ESC Electronic Speed Controller

FIS Fuzzy Inference System

FLC Fuzzy Logic Controller

FPGA Field Programmable Gate Arrays

FST Frenet-Serret Theory

GPS Global Positioning System

HYB Hybrid Control

IADU Integral Absolute Derivative Control Signal

IB Integral Backstepping

IMU Inertial Measurement Unit

I2C Inter-Integrated Circuit

ISE Integral Squared Error

JDBC Java Database Connectivity

LES Large Eddy Simulation

LLA Latitude, Longitude, and Altitude

LQR Linear Quadratic Regulator

MAV Micro Aerial Vehicle

MBPC Model Based Predictive Control

MEMS Micro Electromechanical Systems 
MFR Miniature Flying Robot

MOSFET Metal Oxide Semiconductor Field Effect Transistor

NED North East Down

PD Proportional Derivative

PID Proportional Integral Derivative

PWA Piece-Wise Affine

PWM Pulse Width Modulation

RANS Reynolds Averaged Navier Stokes

RIC Robust Internal-Loop Compensator

RMSE Root-Mean-Square Error

RPM Revolutions Per Minute

SMC Sliding Mode Control

STARMAC Stanford Testbed of Autonomous Rotorcraft for Multi-Agent Control

SPI Serial Peripheral Interface

SQL Structured Query Language

UART Universal Asynchronous Receiver Transmitter

UAV Unmanned Aerial Vehicle

UBL Urban Boundary Layer

VTOL Vertical Take Off and Landing

WSD Wind Simulation Database 


\section{Nomenclature}

$U$ Control law output

$\bar{\Phi}$ Average of absolute difference

$\phi$ Roll angle in earth frame, rad

$\theta$ Pitch angle in earth frame, rad

$\psi$ Yaw angle in earth frame, rad

$p$ Roll rate in body frame, $\mathrm{rad} / \mathrm{sec}$

$q$ Pitch rate in body frame, $\mathrm{rad} / \mathrm{sec}$

$r$ Yaw rate in body frame, $\mathrm{rad} / \mathrm{sec}$

$\omega_{B}$ Angular velocities in body frame, $\mathrm{rad} / \mathrm{sec}$

$X$ Position in earth frame (North) m

$Y$ Position in earth frame (East) $\mathrm{m}$

$Z$ Position in earth frame (Down) $\mathrm{m}$

$u$ Translational velocity in body frame $\mathrm{x}$-axis, $\mathrm{m} / \mathrm{sec}$

$v$ Translational velocity in body frame $\mathrm{y}$-axis, $\mathrm{m} / \mathrm{sec}$

$w$ Translational velocity in body frame z-axis, $\mathrm{m} / \mathrm{sec}$ 
$V_{B}$ Translational velocities in body frame, $\mathrm{m} / \mathrm{sec}$

$J$ Moment of inertia matrix, $k g . \mathrm{m}^{2}$

$M$ Total mass of the quadrotor, $\mathrm{g}$

$M_{o}$ Mass of the center body, g

$m$ Mass of motor, g

$l$ Length of the quadrotor arm, m

$g$ Acceleration due to gravity, $\mathrm{m} / \mathrm{sec}^{2}$

$T$ Thrust, $\mathrm{N}$

$F$ Force, N

$f$ Force component, $\mathrm{N}$

$\tau$ Torque, $\mathrm{Nm}$

$K_{\tau}$ Motor torque constant

$t_{m}$ Motor time constant

$W$ Wind velocity, $\mathrm{m} / \mathrm{s}$

$D$ Disturbance

$B$ Body frame of reference

$E$ Earth frame of reference

$R$ Rotation matrix

$C_{d}$ Drag coefficient 
$S$ Fuselage effective wet area, $m^{2}$

$\rho$ Ambient air density, $\mathrm{kg} / \mathrm{m}^{3}$

\section{Subscript}

$f$ Front

$b$ Back

$r$ Right

$l$ Left

$x$ body frame $\mathrm{x}$-axis

$y$ body frame y-axis

$z$ body frame z-axis

aero Aerodynamic

ref Reference command 


\section{Chapter 1}

\section{Introduction}

\subsection{Motivation}

Over the last decade, quadrotors have been the focus of wide ranging research in university laboratories throughout the world. In particular, quadrotors have emerged as a standard robotic testbed for investigating unmanned aerial vehicles (UAVs) and micro air vehicles (MAVs). Within these research environments, the four rotor helicopters have been miniaturized [1], made to perform aggressive flight maneuvers [2], been learned to navigate [3], played catch [4] and tennis [5], and cooperatively assembled structures [6]. In recent times another new and inevitable phenomenon is taking place: quadrotors are leaving the laboratories and are heading out into the real world. The advantages of the quadrotor design are currently being harnessed into useful tools and employed in many areas of life. The evolution of UAVs geared towards military, civilian, and commercial applications has been very rapid, driven by sectors such as law enforcement [7], agriculture [8], the media [9] and entertainment industries [10], and disaster response [11]. The fact that quadrotor based pizza delivery [12] has become a reality, part of the new era of "drone delivery" [13], clearly attests that the scope of these projects has moved far beyond the most obvious applications into a new realm of possibilities. Furthermore, as off-the-shelf quadrotor platforms continue 
to become readily available to the general public, with some popular models listed in Table 1, the pervasiveness of these drones, as they are commonly referred to, will only increase. As technological advances meet rising demands, the end result is that contemporary quadrotors are becoming ubiquitous in an entirely different and not entirely hospitable territory, the urban landscape.

Table 1: Commercial off-the-shelf quadrotor specifications.

\begin{tabular}{|c|c|c|c|}
$\begin{array}{c}\text { Name and } \\
\text { Manufacturer }\end{array}$ & $\begin{array}{c}\text { Weight } \\
(\mathrm{g})\end{array}$ & Features & $\begin{array}{c}\text { PID } \\
\text { Control }\end{array}$ \\
\hline $\begin{array}{c}\text { Hummingbird } \\
\text { AscTec [14] }\end{array}$ & 910 & $\begin{array}{c}\text { Designed for dynamic } \\
\text { flight maneuvers }\end{array}$ & $\checkmark$ \\
\hline $\begin{array}{c}\text { Phantom } \\
\text { DJI [15] }\end{array}$ & 1300 & $\begin{array}{c}\text { No fly zone restricted } \\
\text { flight }\end{array}$ & $\checkmark$ \\
\hline $\begin{array}{c}\text { Parrot } \\
\text { AR.Drone [16] }\end{array}$ & 410 & $\begin{array}{c}\text { Fully controllable via } \\
\text { smartphone }\end{array}$ & $\checkmark$ \\
\hline $\begin{array}{c}\text { Iris } \\
\text { 3DR [17] }\end{array}$ & 1682 & $\begin{array}{c}\text { Follow mode lets } \\
\text { camera track subject }\end{array}$ & $\checkmark$ \\
\hline $\begin{array}{c}\text { 350 QX } \\
\text { Blade [18] }\end{array}$ & 760 & $\begin{array}{c}\text { Three different flight } \\
\text { modes }\end{array}$ & $\checkmark$ \\
\hline $\begin{array}{c}\text { Scout X4 } \\
\text { Walkera [19] }\end{array}$ & 1770 & $\begin{array}{c}\text { Circle around GPS } \\
\text { waypoint }\end{array}$ & $\checkmark$ \\
\hline $\begin{array}{c}\text { Arducopter } \\
\text { Multiwii [20] }\end{array}$ & custom & $\begin{array}{c}\text { Cross-platform } \\
\text { open source }\end{array}$ & $\checkmark$ \\
\hline
\end{tabular}

There is an archetypical image associated with the downtown of an urban center, exploited as the opening shot of countless blockbuster movies, consisting of myriad high-rise towers and other man-made structures silhouetted against a skyline. This image serves as a visual shorthand illustrating the unique geographical characteristics which differentiate the urban flight environment from the controlled conditions of a laboratory. A typical North American downtown is comprised of a collection of 
buildings with different geometries, and of varying height, length, and width configurations. Such an urban setup includes the existence of individual structures, termed single buildings, as well as urban canyons, which are formed between two or more buildings. For a small sized UAV attempting to navigate the obstacles of such an environment, prevailing wind conditions impose disturbances detrimental to the stability of controls running on the autopilot.

The risks associated with flying UAVs in urban regions are compounded by the high population density within metropolitan areas. Transport Canada charges UAV operators with ensuring the safety of other airspace users, property and persons on the ground [21]. Although no comprehensive database exists tracking incidents related to commercial and recreational UAV usage, news reports detail accidents throughout urban areas, including quadrotor crashes into buildings in Manhattan [22], St. Louis [23], and Vancouver [24]. At this critical phase where many applications require a UAV that can reliably and safely operate in urban environments, there is an urgent need to evaluate the performance of on-board controls in the presence of the type of wind disturbances expected in urban areas. Thus, flying quadrotors in the real world requires a trip back to the research laboratory to fill in gaps in our knowledge about urban winds and suitable control techniques for an urban environment.

\subsection{Problem Statement}

Simply stated, the aim of this thesis is to investigate the windfield present in an urban area, quantify and analyze the effect of these wind disturbances on the flight performance of a quadrotor UAV, and to develop an autonomous position control scheme for flight control under such wind conditions.

In order for any position control technique to be truly tailored for such an environment, it is first necessary to thoroughly identify the nature of the wind disturbances 
anticipated in urban areas. Developing a control technique without considering these acknowledged perturbations, or representing them in the form of random noise or step inputs, may not result in an effective control performance. Hence, this thesis takes an in-depth look at characterizing urban wind conditions and illustrates that the urban wind disturbance is varied in its manifestation, depending mostly on the location of measurement relative to buildings. A freestream wind gust, for example, contains small fluctuations while producing a consistent wind velocity in one particular direction. On the other hand, a wake eddy will contain a three-dimensional flow causing severe fluctuations on all three position control axes. Similarly, a complete reversal of wind flow is observed around the vortices forming close to the building surfaces as well as in its wake. The existence of such varying wind conditions from one location to another a few meters away presents a different challenge for position control techniques, as compared to noise or signal-based generated disturbances.

At present, as mentioned in Table 1, any off-the-shelf rotorcraft contains an autopilot with basic features such as attitude stabilization, GPS-based position hold and waypoint following. The overwhelming majority of rotorcraft use PID control for attitude and position stabilization, unambiguously making it the first candidate control technique to be evaluated for flight performance in urban flows. Based on an extensive literature review, additional established control techniques are also selected for testing. For each of the control methods considered, the performance of its position control in a realistic outdoor simulation will be examined. Hence, operational standards of current controllers are benchmarked for urban flight, with special consideration towards the identification of potential areas in the wake of a building where it is more likely for UAVs to deviate from their desired positions. A quadrotor prototype developed for this thesis is used to perform experimental flights which further validate the simulation findings. 


\subsection{Organization of Thesis}

In Chapter 2, this thesis presents a literature review of several control techniques used for the autonomous flight of quadrotors. The aim is to identify methods which have been successfully employed in the flight control of quadrotors with supporting experimental results. The review also confirms whether any outdoor flight control has been conducted in the presence of real urban wind disturbances.

Chapter 3 details the methodology adopted to develop an accurate flight simulator to model and incorporate urban wind conditions. Several points of interest around a single building are presented with a discussion about their prevailing wind conditions. An evaluation criteria is also established to judge and compare the flight performances of different control techniques in a consistent test setup.

Details of the control techniques chosen for assessment are presented in Chapter 4. Using each selected method, control laws are derived for the position stabilization of a quadrotor UAV.

The results of the flight simulations are presented in Chapter 5, with a consistent setup defined and repeated for each of the control techniques. These results are evaluated by the earlier-specified criteria and comparisons are drawn and analysed between the performance of the different control methodologies.

Based on the analysis of flight control performance in urban wind conditions, Chapter 6 proposes a novel control scheme for autonomous position control of a quadrotor in the presence of wind disturbances. Simulation and experimental results are presented to validate the flight performance of the proposed control scheme.

The final conclusions of this thesis, along with future research directions, are outlined in Chapter 7. 


\section{Chapter 2}

\section{Literature Review}

\subsection{Autonomous Flight Control of Quadrotors}

Since the inception of the quadrotor design named Bréguet Richet Gyroplane No. 1 [25] [26] by two brothers named Louis and Jacques Bréguet, quadrotors have kept an interested following. However, in recent times, quadrotor Unmanned Aerial Vehicles (UAVs) have gained a tremendous interest from the research community. Owing to its Vertical Take-off and Landing (VTOL) and impressive maneuverability, particularly in hovering and traversing through an environment with many obstacles, the quadrotor is a preferred choice as UAV platform. Extensive research has been conducted on realistic modeling and control of a quadrotor, and test-beds have been constructed in various research projects, where flight simulators are also developed for testing the control laws beforehand.

Quadrotors are proving to be a preferred choice in the research involving multiple UAVs and their coordination and cooperation. The Stanford Testbed of Autonomous Rotorcraft for Multi-Agent Control (STARMAC) [27] is one such popular research project. Multiple quadrotors are used outdoors for tasks such as solving the decentralized collision avoidance problem, and automated search and rescue, where 
UAVs exchange their observations with each other to track the location of a target beacon. Another project at MIT called the UAV SWARM Health Management Project [28] involves multiple UAVs performing complex mission scenarios with autonomous recharge. This research by Bethke et al. [29], also includes cooperative vision-based estimation and tracking of objects of interest using multiple UAVs. Recently, a research project at the University of Pennsylvania called GRASP Multiple Micro UAV Testbed [30] has gained some attention. In this project multiple UAVs are used to perform team based tasks such as lifting and moving of objects cooperatively. The localization information is provided by a Vicon motion capture system that consists of a set of cameras in the room for monitoring the UAVs at high speed. Based on a similar setup, Ritz et al. [4] from ETH Zürich demonstrated the cooperative catch and throw of a ball using a net by a fleet of quadrotors. The UAVs used in both the GRASP and ETH projects are Hummingbird quadrotors from Ascending Technologies $\mathrm{GmbH}$, which can perform fast acrobatic maneuvers.

Many control techniques are proposed in the literature for the autonomous flight control of quadrotors. In some recent research work, uncertainties such as noise and wind disturbances are considered and control techniques are proposed with some experimental results. The next sections present some of the prominent control techniques for the flight stabilization and control of quadrotors found in the literature including proportional-integral-differential (PID), feedback linearization, backstepping, sliding mode, linear quadratic regulator (LQR), $\mathcal{H}_{\infty}$, adaptive control, fuzzy logic, neural networks, and reinforcement learning.

\subsection{PID Control}

Proportional-Integral-Differential (PID) is one of the most common feedback control techniques. PID is popular in control systems due to its simple implementation and 
ability to produce a control response suitable for many industrial applications. PID is implemented by first determining the control error, $e$, which is the difference of a set point (reference variable) and the measured process variable. The control signal $u$ is given as:

$$
u(t)=K_{P} e(t)+K_{I} \int_{0}^{t} e(t) d t+K_{D} \frac{d e(t)}{d t}
$$

where $K_{P}, K_{I}$, and $K_{D}$ are the controller parameters referred to as proportional, integral, and derivative gains, respectively. The proportional term is the present control action, whereas the integral and differential terms are the past and future (predictive) control actions, respectively.

Bouabdallah [31] designed multiple PID controllers for the attitude stabilization of a quadrotor. The gyroscopic effects resulting from propeller rotation coupled with rigid body rotation are neglected, thereby removing the cross coupling. First, a PD controller is realized and tested in simulations with satisfactory performance. Later an integral term is introduced to eliminate the steady-state error on a real tethered quadrotor. It is shown that this classical control technique can be adopted for efficient attitude stabilization in a hover condition. The quadrotor is able to perform autonomous near hover flight in the absence of large disturbances using the developed PID controllers. However, it is asserted that in the presence of strong perturbations (such as wind) this controller would not be able to stabilize the quadrotor.

A heavy quadrotor prototype weighing more than four kilograms is designed and developed at Australian National University (ANU) and several papers by Pounds et al. [32], [33], [34] have been published in past few years. In a recent paper [35], a dynamic model of the quadrotor is developed and rotor dynamics and effects such as blade flapping are considered in the system model. Unforced stability analysis is conducted in order to optimize the airframe geometry pertinent to the placement of rotors with respect to the center of gravity and to make the system controllable. 
Stability derivatives are also derived for the fixed pitch rotors used in the propulsion units. It is suggested that an uncomplicated control technique such as PID may offer simplicity and robustness to parameter variations. A PID controller is therefore implemented on the discretized model of the system. In order to test the controller performance, a tethered flight experiment is conducted first using a gimbal rig and slower rotor speeds. The quadrotor is able to stabilize its attitude within $\pm 1^{o}$ of level precision. Later, an outdoor autonomous flight experiment is conducted where the quadrotor is set untethered and allowed to take-off under manual control. To test the autonomous attitude control the quadrotor control is then switched over to the onboard flight controller for 25 seconds. The experimental results show that the proposed PID controller is able to achieve a $\pm 1^{\circ}$ of level precision for about 5 seconds. The overall flight time is insufficient to confirm a stable hover performance specifically in the presence of outdoor wind disturbances.

An additional PD control loop based on brushless DC motor armature current feedback is combined with a state feedback control to address the attitude stabilization of a quadrotor by Sanchez et al. [36]. This different approach is based on the fact that the four propulsion units (usually off the shelf components) of a quadrotor are not the same and therefore require additional control to guarantee equal performance from each propulsion unit. The majority of the previous control techniques overlooked this issue and consider such inequalities as disturbances which are left for the attitude controller to handle. The experimental results demonstrate an improved attitude stabilization of $\pm 2^{\circ}$ oscillations from the desired value despite introduced perturbations. Also, the settling time of the attitude controller is observed to be impressively fast with values of less than a second. The improvement in the attitude angles provided by the proposed novel technique open doors for increasing autonomy of quadrotor. Furthermore, a new method to compute the robustness margin of the PD controller is also proposed in [37]. 
In a paper by Tayebi and McGilvray [38], the attitude stabilization of a VTOL quadrotor is explored. Two controllers are designed, first a model-dependent PD2 which takes the coriolis and gyroscopic torques into consideration. The second is a classical PD (model-independent) controller where the coriolis and gyroscopic torques are not included in the model. Motor torque models are also derived and a control law for stabilization is applied. Lyapunov stability proofs are derived to show the asymptotic stabilization of the proposed control laws. The experimental setup is conducted on a readymade DraganflyerIII that is modified by adding low-cost inertial sensors and control circuitry. The system is gimbaled and interfaced with real-time workshop (Matlab) using dSPACE for control law application. Noise filtering is also performed via software filters in Simulink. Three experiments are conducted to prove the stability and control law convergence and compared with other controllers. Results conclude that a global exponential attitude stabilization of a quadrotor can be achieved.

Park et al. [39] use Robust Internal-Loop Compensator (RIC) based disturbance compensation combined with PID for attitude and position control. The test experiments are conducted indoor without any disturbances. A stable flight performance is achieved using $\mathrm{RIC}$ with $\pm 5^{\circ}$ oscillations and 1.5 sec settling time. Improving on the idea, Park et al. [40] propose a disturbance observer based controller combined with a PID controller for the altitude and attitude control. Experimental results for indoor hovering of a quadrotor show an improved attitude stabilization with $\pm 2^{\circ}$ of error while maintaining the desired altitude with $\pm 0.05 \mathrm{~m}$ error.

In addition, a substantial amount of further literature is available where PID control is successfully used for the flight control of a quadrotor. However, these studies are either conducted on tethered testbeds, in indoor flights, or consist solely of simulations, and are therefore less pertinent to the goals of the research at hand. The experimental studies include Hanford [41], [42] for the attitude control of a quadrotor 
on a Whitman training stand. Hoffmann et al [43], and Waslander et al [44] for trajectory tracking. Altug and Tarhan [45] for the stabilization of a quadrotor, where an Extended Kalman Filter (EKF) is also implemented for the estimation of the attitude angles using a catadioptric camera. Simulation studies are conducted by Orsag and Bogdan [46], where blade element theory for rotor modeling is developed. Salih et al. [47] and Weng et al. [48] consider the attitude stabilization of a quadrotor. Vision based PID control research include Fowers et al. [49], Altug et al. [50], Cabecinhas et al [51], and Wenzel et al. in [52] and [53].

Another approach to control nonlinear systems is to transform them into equivalent linear system by changing system variables and applying suitable control input. This approach is called feedback linearization, which makes it possible to cancel undesirable dynamics of a nonlinear system and replace them with a desirable linear dynamics. For a given nonlinear control system,

$$
\begin{gathered}
\dot{x}=f(x)+g(x) u \\
y=h(x)
\end{gathered}
$$

where $f(x), g(x)$, and $h(x)$ are nonlinear but smooth functions and $x(t) \in \mathbb{R}^{n}$ is the vector of state variables, $u(t) \in \mathbb{R}^{p}$ is the vector of manipulated input variables, and $y(t) \in \mathbb{R}^{m}$ is the vector of controlled output variables. A state feedback control law,

$$
u=a(x)+b(x) v
$$

is developed such that the new input variable $v$ and the output $y$ form a linear system.

Feedback linearization based control is used for attitude stabilization of a Qball-X4 
quadrotor along with PID control for trajectory following by Zhou et al. [54]. Reconfigurable control allocation for fault tolerant systems using a fixed-point method is also implemented in conjunction with the attitude and trajectory control. Simulations are carried out to test the controllers in fault-free conditions, as well as during partial loss of one actuator. Simulation results show that the Qball-X4 is able to stabilize the attitude within 5 seconds once the fault is introduced. The trajectory following performance is observed to be slow with maximum deviations of approximately $7 \mathrm{~m}$ from the desired trajectory without any fault. In the case of introduced fault on an actuator, the respective position deviates to $100 \mathrm{~m}$ and the controller is not able to bring it back while the attitude is maintained at zero during 90 seconds of flight time.

Aerodynamic effects such as blade flapping and total thrust variation in translational flight of a quadrotor are investigated by Hoffmann et al. [55]. A novel feedback linearization controller is proposed to deal with the aerodynamic effects in consideration. The attitude control is achieved using a standard PID controller augmented with feedback on angular accelerations. A stall turn maneuver which consists of a $8 \mathrm{~m} / \mathrm{s}$ high-speed forward flight followed by a reversal of attitude command is performed to test the effectiveness of the proposed control technique on a quadrotor testbed called STARMAC. The experimental flight results show attitude stabilization with $\pm 3^{\circ}$ of tracking error while maintaining the altitude within $\pm 0.2 \mathrm{~m}$ of the desired value.

\subsection{Lyapunov-based Methods}

Bouabdallah et al. [56] use Lyapunov control theory for the attitude stabilization problem. Using LaSalle invariance theorem, asymptotic stability is ensured by starting from a point in a set around the equilibrium point. Global stability is also ensured in the defined case. The controller is tested both in a simulation, as well as in an experiment to stabilize the attitude angles of a tethered quadrotor. The developed 
controller proves to be very reactive and is able to maintain the attitude angles to the desired zero value despite of large initial conditions within 5 seconds. Autonomous hover flight is not achieved despite good rejection of disturbances by the developed control.

In a paper by Dzul et al. [57], the real-time attitude stabilization and tracking of desired trajectories of a quadrotor is explored. Two types of control laws are designed and implemented. The first is a simple PD control law for the altitude and yaw control. The second is a non-linear control based on nested saturation. The stability proof is not provided, however it is stated that the global stability is proved. The attitude stabilization is achieved, however, the inclusion of already existing gyro based stabilization on the DraganFlyer is not mentioned. A secondary Inertial Measurement Unit (IMU) called POLHEMUS is added for stabilization. Experimental results are presented showing the stabilization achieved.

Backstepping control which recursively applies Lyapunov stability for systems in strict feedback form, is used for the attitude stabilization of the quadrotor by Bouabdallah [31]. The control law is synthesized to force the system to follow a desired trajectory. The proposed backstepping controller is tested both in simulations as well as on the tethered quadrotor with satisfactory performance where stabilization is achieved within five seconds from an initial condition of 32 degrees of roll angle. Despite impressive stabilization of the attitude angles in the presence of large disturbances, the backstepping controller is not successful in achieving autonomous hover flight with a failure rate of $50 \%$.

Vries and Subbarao [58] use backstepping based nested multi-loop for hover control. Simulations are conducted with triangular shaped wind gust disturbances. Position control is achieved using a very simplified dynamic model without aerodynamic, rotor, and sensor modeling.

The backstepping control technique is combined with an integral action from a 
conventional PID control by Bouabdallah [59]. The idea is to bring together the robustness against disturbances of backstepping controller, with the robustness against model uncertainties of integral action. The result of this work is an Integral Backstepping (IB) control scheme used for both attitude stabilization and position control. The stability analysis is performed using Lyapunov theory and global stability is ensured by a positive definite candidate function and its derivative being less than zero. The simulation including an actuator dynamic model with amplitude saturation and experimental results of autonomous hover shows attitude stabilization with oscillations between $\pm 5^{\circ}$. The oscillations are attributed to the slow dynamics of the actuators coupled with the differences in the propulsion units. Position control is achieved with an accuracy of $3 \mathrm{~cm}$ for altitude control in simulation. Similarly, waypoint sequencing is also carried out in simulation with a maximum deviation of $20 \mathrm{~cm}$.

Integral-Backstepping control technique is compared against a PID controller designed for the attitude stabilization of a tethered quadrotor on a gimbal testbed by Hoffmann et al. [60]. Both controllers are tested for robustness and disturbance rejection by conducting simulations and experiments. Wind gusts are simulated in the form of a sequence of impulses added to the control in hovering state. The disturbances introduced cause attitude errors ranging $16^{\circ}$ to $25^{\circ}$ which are successfully compensated by the PID controller within a second with an overshoot of 6 degrees. In experiment the quadrotor is exposed to a constant wind of $8 \mathrm{~m} / \mathrm{s}$ while tracking a sinusoidal attitude reference command. The integral backstepping is able to stabilize the attitude with an overshoot of $2^{\circ}$ in the absence of wind, and $4^{\circ}$ of overshoot in the presence of wind. The robustness of both controllers is evaluated by adding a weight mounted on one of the arms of the quadrotor, thereby varying the payload as well as the inertia. A $90 \mathrm{~g}$ added weight causes an attitude error of $8^{\circ}$ that is compensated within 5 seconds upon the introduced weight by the PID controller. For testing robustness of the IB controller a $330 \mathrm{~g}$ weight is added on top of the center of quadrotor 
airframe resulting in the CoG shifting above the airframe center thus making the system unstable. The results of reference tracking show satisfactory performance with slight increase in rise time as well as in overshoot. Yet the controller remains stable and is able to handle parameter uncertainties despite degraded conditions.

A hybrid control technique based on combining backstepping with Frenet-Serret Theory (FST) is developed to address the attitude stabilization of a DraganFlyer quadrotor by Colorado et al. [61]. The proposed control technique introduces estimation of the desired angular accelerations as a function of quadrotor flight velocity. An aerodynamic model based on lift and drag forces and blade airfoil theory are also included in the system model for simulation purposes. Simulations and experiments are performed to compare the performance of the proposed control methodology against a conventional PID controller. Results show the improvement achieved by the introduction of desired angular acceleration command which responds immediately to any abrupt change in the angular rate. In error tracking the backstepping-FST controller proves to be 2.5 times better in performance than the PID controller while performing maneuvers at a high speed of $2 \mathrm{~m} / \mathrm{s}$.

Backstepping control has been used in several other studies where the effectiveness of this control technique is demonstrated in simulation, such as by Mian and Daobo [62], Adigbli [63], Hamel et al. [64], and experimentally by Guenard et al. [65], Hamel et al. [66]. In some cases, such as by Zuo [67], Guerrero [68], and Altug [69] external disturbances are introduced to prove the robustness of the Backstepping control. However, none of these disturbances represents the wind gust conditions that are present in realistic outdoor environments.

Sliding Mode Control (SMC) is another nonlinear control technique used to deal with the parametric and modeling uncertainties of a nonlinear system. SMC also uses the Lyapunov stability method for stable control of nonlinear systems. Using SMC a higher order system is transformed into a first order system that is easier to control 
using simple control algorithms. The main strength of SMC is in its robustness. For a given nonlinear system,

$$
\dot{x}(t)=f(x, t)+g(x, t) u(t)
$$

where $x(t)$ is an $\mathrm{n}$-dimensional state vector such that $x(t) \in \mathbb{R}^{n}, u(t)$ is an mdimensional input vector such that $u(t) \in \mathbb{R}^{m}$ and functions $f(x, t)$ and $g(x, t)$ are smooth continuous for which a unique solution is assumed to exist. A state feedback law $u(x(t))$ is designed to map the state $x(t)$ at time $t$ to the input $u$.

The sliding mode control is based on the selection of a sliding surface so that the system trajectory shows desirable behaviour within the selected surface. High feedback gains are tuned such that that system is forced to remain on the sliding surface.

A sliding mode controller is designed for the attitude stabilization of a tethered quadrotor by Bouabdallah [31]. Simulations and experiments are performed to test the performance of the proposed controller. The results show stabilization achieved within 8 seconds from an initial condition of $45^{\circ}$, however, a shattering effect is observed on all three rotation angles. Due to its switching nature not adapting to the dynamics of the quadrotor, the sliding mode controller is unable to achieve autonomous hover.

Hoffmann et al [70] use sliding mode control for the altitude control of a quadrotor testbed called STARMAC. The Lyapunov proof is also derived to guarantee the asymptotic stability of the control scheme. The attitude controller is based on LQR approach and its cost matrix is chosen in order to penalize the angular deviations more than the angular rates. However, this results in a degraded flight performance at higher thrust levels. To address this issue, the control effort is softened by lowering the cost matrix values for the angular deviations. Experimental results of an outdoor 
test flight show that the quadrotor is able to maintain an altitude of $1 \mathrm{~m}$ with \pm 0.25 $m$ deviations. The deviations are caused due to the change in net vertical thrust while changing attitude angles, thus showing the need for a more effective control approach.

In another experimental study by Zhang et al [71], a PD sliding mode is used for the attitude and altitude control of a quadrotor in the presence of introduced disturbances by physically changing the attitude during flight. A tracking differentiator is also used for the estimation of the velocities via vision based Vicon positioning system. The PD control is sufficient for the attitude stabilization, however, it cannot deal with disturbances beyond certain levels. Therefore, a sliding mode control is proposed in order to deal with such disturbances and its Lyapunov stability-proof is also derived. The results of an indoor test flight show acceptable position control that is plagued by erroneous readings from the Vicon positioning system. The attitude control is achieved with $\pm 4^{\circ}$ deviations and a minor drift visible on roll and pitch axes. The proposed controller is able to stabilize the attitude when perturbation is manually introduced on pitch or roll axis during flight.

Sliding mode control is proposed and simulation studies are conducted by $\mathrm{Xu}$ et al. [72], Bouadi et al. [73], and Efe [74]. However, none of these studies demonstrate performance of their controllers in presence of disturbances. Benallegue et al. [75], on the other hand, added sinusoidal aerodynamic forces and moments in the system model and simulation results demonstrate the ability of sliding mode control to deal with such sinusoidal disturbances. 


\subsection{Optimal Control}

Linear Quadratic Regulator (LQR) is an optimal control technique focused towards operating a dynamic system at minimum cost. For a continuous-time linear system,

$$
\dot{x}=A x+B u
$$

and

$$
y=C x
$$

One can formulate this as an optimization problem and define a quadratic cost function,

$$
J=\int_{0}^{\infty}\left(x^{T} Q x+u^{T} R u\right) d t
$$

where $Q$ is a positive semi-definite symmetric state weighing matrix and $\mathrm{R}$ is a positive definite symmetric control weighing matrix.

Now define a feedback control law,

$$
u=-K x
$$

and choose $\mathrm{K}$ such that the cost function is minimized.

$$
K=R^{-1} B^{T} P
$$

where $P$ is determined using Algebraic Riccati Equation (ARE).

$$
A^{T} P+P A-P B R^{-1} B^{T} P+Q=0
$$


Using optimal control theory, Bouabdallah et al. [76], developed an adaptive optimal controller using LQ control technique. The controller is tested twice in simulations using a permanent and variable solution to the Riccati equation. A full model of the system including the actuator dynamics is used in the simulations. The variable solution with a fixed final condition based LQ controller shows better results due to the optimization of the cost function for every sub-trajectory. Stabilization is achieved within 4 seconds from initial angles values of up to $45^{\circ}$. Overall an average performance is achieved by the controller with the presence of steady state errors on all attitude angles. The degraded performance is believed to be due to the fact that the actuator dynamics are not considered in the development of the proposed LQ controllers. Due to the presence of steady state errors and lacking dynamics the LQ control is unable to achieve autonomous hover flight.

A direct method based control technique is used for real-time planning and following of a flight trajectory of a DraganFlyer quadrotor by Cowling et al. [77]. The direct method using inverse dynamics, allows for the satisfaction of the higher order derivatives at both boundary points and it can use any model and performance index. An LQR based optimal controller is implemented for the trajectory following in this research. Simulations are performed using a detailed dynamic model of the system where most of the parameters of the quadrotor are identified experimentally. The wind is modeled as a small translational drift of $0.1 \mathrm{~m} / \mathrm{s}$ and random noise is added to represent gust conditions. The results show the feasibility of the proposed method by achieving optimal following of the generated trajectories in real-time despite the presence of these disturbances. In assessing the significance of these results it is important to note that a wind disturbance of $0.1 \mathrm{~m} / \mathrm{s}$ with zero mean and $0.01 \mathrm{~m} / \mathrm{s}$ variance is small as compared to actual anticipated wind velocities in urban areas (which can for example range up to $5.68 \mathrm{~m} / \mathrm{s}$ when a freestream wind of $4 \mathrm{~m} / \mathrm{s}$ enters an urban area). 
A constrained finite time optimal (CFTO) control is used by Alexis [78], [79] for set-point maneuvers of a Draganflyer quadrotor tethered by a Heli-Safe flight test stand. This optimal control technique extends the previous list of control methodologies proposed for autonomous flight control of the quadrotor. The nonlinear dynamics of the quadrotor are linearized about various operating points yielding a set of Piecewise Affine (PWA) models. For each operating point the quadrotor is assumed to be operating within a range of angular rates. The developed model also considers the actuator constraints such as saturation. In order to test the performance of the CFTO controller proposed for the stabilization of the attitude angles, simulations and experiments are conducted in the presence of wind disturbances. In simulation the wind turbulence is introduced using von Karman forming filters. In experiments, two different wind conditions are tested; first a laminar flow and later wind gusts with velocity magnitudes of $1.31 \mathrm{~m} / \mathrm{s}, 3.84 \mathrm{~m} / \mathrm{s}$, and $1.65 \mathrm{~m} / \mathrm{s}$ are applied on the $\mathrm{x}$, $\mathrm{y}$, and $\mathrm{z}$ axis respectively using an electric fan. The experimental results demonstrate the ability of the CFTO controller to achieve the desired set-points while rejecting the introduced wind disturbances. The proposed methodology is based on piecewise affine systems and to achieve higher angular velocities and reducing the steady state error, it is desirable to increase the number of PWA systems. The trade-off is the exponentially increased complexity of the controller due to the larger dimension of state vector as well as higher number of constraints. This results in difficulty for real-time implementation on embedded platforms, hence increasing the cost of the system both computational and resource wise.

\subsection{Robust Control}

Robust controllers are designed to explicitly deal with bounded uncertainties in a system. A robust control guarantees that if the uncertainties are within defined limits 
the fixed control law remains effective. Few robust control techniques are applied towards flight control of a quadrotor. In this section $\mathcal{H}_{\infty}$ control for quadrotor flight control is presented.

In the work by Raffo et al. [80], a hierarchical scheme is presented which consists of an integral model predictive controller for path following combined with a nonlinear $\mathcal{H}_{\infty}$ controller for attitude stabilization. Lagrange-Euler equations are used for the dynamic modeling where gyroscopic effects from the propellers are not modeled and considered as disturbances on rotational axes. Aerodynamic forces and moments are also considered as external disturbances. The control methodology is comprised of two control loops. In the outer loop error model, a state space based predictive controller with integration of the position error is used for the control of translational motions. Implementation is carried out in two stages where altitude is controlled first and references for the roll and pitch angles are generated in the second stage. In the inner loop the $\mathcal{H}_{\infty}$ controller is used for attitude stabilization for the stability of which a Lyapunov proof is also provided. Simulations are carried out and compared against a backstepping controller in the presence of external disturbances. Persistent gusts of wind are applied to the quadrotor during flight simulations by adding additional forces and moments in the equations of motion. Performance indexes such as Integral Square Error (ISE) and Integral Absolute Derivative control signal (IADU) are utilized for quantitative comparison between the proposed control technique and backstepping control. ISE shows a performance increase ranging $30 \%$ to $40 \%$ for the translation motion, $40 \%$ to $70 \%$ for roll and pitch angle and approximately $12 \%$ for yaw angle stabilization. IADU shows an improvement in the smoothness of the control signal by $15 \%$ for thrust and $56 \%$ to $70 \%$ for the torques applied.

A robust $\mathcal{H}_{\infty}$ control design is presented by Kis et al. [81] for the attitude stabilization of a quadrotor based only on angular rates. This is done in order to emulate situations where other sensors fail, such as GPS and vision. The performance of the 
$\mathcal{H}_{\infty}$ controller is compared against a LQ state feedback controller. Both simulation and experimental flight tests are conducted in no wind conditions with the presence of sensor noise as a disturbance. The LQ controller results in a small steady state error while stabilizing the attitude of the quadrotor with $\pm 2^{\circ}$ oscillations. The $\mathcal{H}_{\infty}$ controller manages to bring these oscillations to $\pm 1^{\circ}$ but a very small steady state error remains on the attitude angles causing lateral drift.

Mokhtari et al. [82] developed a feedback linearization control combined with a linear $\mathcal{G H}_{\infty}$ control for a robust flight control of a quadrotor. The feedback linearization technique is based on the Sobolev norm, which transforms a nonlinear system into its tangent linearized system around an operating point. The $\mathcal{G} \mathcal{H}_{\infty}$ control law ensures that the compensated system is asymptotically stable even in the presence of external disturbances. Simulations are conducted where constant aerodynamic forces and moments are introduced as external disturbances. Results show a better performance by the flight controller when only the aerodynamic forces are applied. The proposed controller shows sensitivity for the roll and pitch control when aerodynamic moments are introduced and the overall settling time is noticeably significant.

Chan and Huzmezan [83] propose an $\mathcal{H}_{\infty}$ loop shaping controller for the attitude stabilization of a tethered quadrotor. Also, a constraint model based predictive controller (MBPC) is designed for longitudinal and lateral trajectory control. In the proposed combined $\mathrm{MBPC} / \mathcal{H}_{\infty}$ scheme, the inner loop based on $\mathcal{H}_{\infty}$ is intended for a robust stabilization and disturbance rejection while in the outer loop MBPC ensured a good performance for position control even during large maneuvers. The controller is tested in simulation first and later on a DraganFlyer III quadrotor tethered on a custom designed flying mill. Results show a 3 second settling time for the attitude controller in response to an introduced step disturbance. The position control is also achieved in simulation test with a settling time ranging 4 to 5 seconds for a desired deviation of 40 to 60 meters. 


\subsection{Adaptive Control}

Contrary to robust control, adaptive control seeks to change the control law in the presence of uncertainties without prior knowledge about the bounds on the uncertainties. There exist several adaptive control techniques and presented below are few selected ones that are used for the flight control of a quadrotor.

A novel nonlinear adaptive control technique based on backstepping is proposed by Huang et al. [84] for the trajectory tracking of a quadrotor with mass uncertainty. The dynamic model of the quadrotor is divided into four sub-systems comprising one under-actuated sub-system, two fully actuated sub-systems and the last one for the dynamics of the thrust forces. A backstepping technique is used in conjunction with a parameter adaptive design to develop an adaptive tracking controller. Lyapunov based stability analysis is done to prove that asymptotic tracking can be achieved while making mild assumptions about the roll and pitch angles of the quadrotor. Simulations are carried out to demonstrate the tracking performance of the proposed controller. Simulation results show acceptable performance of the proposed adaptive control design with settling time of 4 seconds for a position displacement of $0.5 \mathrm{~m}$. The yaw angle stabilization is poor as it required same 4 seconds from an initial angle of $30^{\circ}$.

Similarly, in another approach by Madani and Benallegue [85], a nonlinear adaptive controller based on backstepping is proposed and neural networks are used to compensate for any unmodeled dynamics such as aerodynamic effects. Lyapunov stability is used for the controller design and simulations are conducted to test the controller performance. The proposed controller shows robustness in the tested event of sudden change in the quadrotor's mass, however, no external disturbances are considered in the simulations. The settling time of 8 seconds for the desired position control is comparatively slower than another robust adaptive control technique 
proposed by Nicol et al. [86].

Coza and Macnab [87] propose an adaptive fuzzy controller for the attitude stabilization of a quadrotor in the presence of wind disturbances. It is argued that traditional adaptive control for robust parameter update might be insufficient for the fuzzy scheme. Techniques such as e-modification are prone to persistent oscillations in the inputs that result in large drifts in the fuzzy membership centers. In the proposed work a set of alternate membership centers guide the adaptation process to prevent any drift. Simulations are carried out to compare the performance of the proposed technique with the e-modification method. The simulation results show similar performance of both techniques but in the case of wind disturbances the e-modification results in the expected drift in the membership centers whereas the proposed controller is able to prevent such undesired drifts thus showing robustness to disturbances.

A self-tunable zero-order Takagi-Sugeno fuzzy inference system is proposed by Zemalache and Maaref [88] for the flight control of a quadrotor. Fuzzy logic is used in a multi-layered neural network with each layer connected to another by adjustable parameters. The optimization of the parameters is achieved by back-propagation of the gradient to minimize the cost function. Simulations are performed while taking into account external disturbances, such as a constant aerodynamic drag force of $2.1 \mathrm{~N}$ applied in the opposite direction of the flight. The performance of the proposed control technique is compared with classical model based methods, such as static feedback linearization and backstepping. The position control is successfully demonstrated by the self-tuning controller with a settling time of 4 seconds.

Sastry et al. [89] propose an adaptive sliding mode controller for the autonomous flight control of a quadrotor. The proposed controller uses input augmentation in order to deal with the under-actuated dynamics, sensor noise and external disturbances without saturating the control inputs. The adaptive sliding mode controller is 
tested in simulations and its performance is compared against a feedback linearization technique. The proposed controller demonstrates good tracking performance even in the presence of introduced sensor noise and aerodynamic ground effects. The settling time of the controller is 10 seconds for $10 \mathrm{~m}$ desired displacement, and 5 seconds for attitude stabilization from $30^{\circ}$ initial angle. On the other hand, the feedback linearization based controller shows sensitivity to the introduced disturbances thus validating the effectiveness of adaptive control for such cases.

\subsection{Intelligent Control}

Fuzzy control is a special kind of nonlinear control technique successful in dealing with systems that are subjected to uncertainties. Fuzzy controllers are designed intuitively in the light of expert knowledge acquired on the behaviour of the system in concern. The knowledge base is often based on experience, human-like reasoning and common sense, regardless of the mathematical model of the dynamics of a system. Fuzzy control is based on fuzzy logic and thus fuzzy set theory which deals with sets without crisp boundaries and without binary membership characteristics. A

fuzzy set may be represented by a membership function $\mu_{A}(x)$ that gives degree of membership $\mu_{A}\left(x_{i}\right)$ within the set $A$, of any element $x_{i}$ of the universe of discourse $X$. Linguistic variables are used to represent membership functions to mimic humanlike language. Simple IF-THEN implications (rules) are used to solve a given control problem. A Compositional Rule of Inference (CRI) is utilized to make inferences using a composition operation along with a rule-base. By applying CRI, a fuzzy control action is:

$$
\mu_{C}=\operatorname{maxmin}_{Y}\left[\mu_{Y}, \mu_{R}\right]
$$

where $Y$ is the fuzzified input, $R$ is the knowledge base, and $C$ is the control action. Fuzzy control is used for the autonomous flight control of a quadrotor by Raza and 
Gueaieb [90]. Six identical fuzzy controllers are implemented to achieve the position and attitude control simultaneously. Each controller requires two inputs of state error and error rate while producing a single control action. A unified rule base is developed which is comprised of nine IF-THEN rules used first with a Mamdani and later with a zero order Takagi-Sugeno-Kang (TSK) inference engine. In the proposed control strategy the desired roll and pitch angles are continuously anticipated by the $\mathrm{x}$ and $\mathrm{y}$ position fuzzy controllers. Therefore, once the desired position is achieved the attitude is stabilized afterwards. The inputs to the fuzzy controllers are pre-processed and its output post-processed to calculate the pulse width modulation (PWM) value for each individual motor. Simulations are performed to test the performance of the two proposed flight controllers in the presence of disturbances such as sensor noise and wind gust conditions based on sinusoidal signal of $10 \mathrm{~m} / \mathrm{s}$ north-east wind velocities. The results show an attitude stabilization with a $\pm 5^{\circ}$ oscillations and settling time of 5 seconds from an initial angle of $25^{\circ}$. The position control is smooth and stable with a settling time of 15 seconds for a $25 \mathrm{~m}$ desired reference value.

In a similar approach, Santos et al. [91] use fuzzy control for the flight control of a quadrotor. Four fuzzy PID-like controllers are implemented to control the altitude and the three attitude angles. The inputs to the controllers are the state error, error rate and integral of the error. Gains are applied to the three inputs of the controller. The output control action term is also passed through a gain and post processed to generate the desired power for each of the four motors. Mamdani inference with min-min operators is used with centroid of area defuzzification. The gains are tuned based on trial and error and simulations are performed to evaluate the performance of the proposed control technique. No disturbances are accounted for in the simulations, either for sensor noise or wind conditions. Simulation results show altitude control and attitude stabilization with a settling time of 1.5 seconds and a relatively smooth and stable performance for the altitude and yaw angle control when compared with 
the control of roll and pitch angles. Simulations to evaluate the trajectory control performance are also conducted with satisfactory results.

Yapici [92] proposes a self-ruled fuzzy controller for the flight control of the quadrotor. In the proposed structure the controller has no rule base and no membership functions, which is different as compared to conventional fuzzy controllers. The proposed controller structure is based on fuzzy logic concepts and human decision making and instead of rule base and membership functions a mapping curve is used. The mapping curve assigns logical values to the input and output variables and the rules are essentially present in the structure of the controller. The final control surfaces obtained for the six controllers used for the attitude and position control match that of any conventional fuzzy-PD controller. Simulation tests are conducted on an inverted pendulum first and later on a nonlinear dynamic model of a quadrotor. No aerodynamic effects, sensor noise, or wind disturbances are taken into account. Simulation results show acceptable position control with a settling time of 3 to 4 seconds for reference deviations of 3 to 4 meters respectively. The attitude is stabilized in less than a second for a reference angle of $30^{\circ}$. Overall, a stable controller performance is demonstrated.

Fuzzy logic is used for the scheduling of backstepping controller parameters to achieve attitude stabilization of a quadrotor by Younes and Jarrah [93]. Lyapunov based stability is ensured around a fixed set-point using the backstepping control technique. The proposed backstepping fuzzy logic (BFL) controller is compared against a simple backstepping controller. The experimental results for a tethered quadrotor show that the BFL controller is able to perform better attitude stabilization than the backstepping controller. The attitude is stabilized within 2 seconds, however, no external disturbances are considered in the experiments.

Waslander et al. [27] present a comparison of reinforcement learning and integral sliding mode control techniques for the autonomous altitude control of a quadrotor. 
Contrary to $\mathrm{x}$ and $\mathrm{y}$ position control that can be reliably achieved by implementing a carefully designed integral LQR, the altitude control is proved to be a nonlinear control problem for which classical linear techniques fail to provide sufficient stability. Disturbances such as sensor noise, blade flex, ground effect, battery discharge dynamics and complex airflow generated by the rotors induce destabilizing effects in free flight. The integral sliding mode control starts with an assumption that disturbances cannot be modeled and therefore a robust control law is needed to deal with a defined range of expected disturbances. Another nonlinear control approach called model-based reinforcement learning is used to develop a dynamic model based on input-output recorded values and then optimal control law is applied to the learned model. Experimental results for both control techniques showed similar performance with a response time of 1 to 2 seconds and a 5 second settling time without any steady state error. The integral sliding mode control show slightly better transient dynamics than the reinforcement learning control. Autonomous hover is performed with a position response maintained within a 3 meter circle by using integral sliding mode control for altitude combined with integral LQR control for lateral position.

Reinforcement learning is applied for the attitude stabilization of a quadrotor by Voos et al. [94]. The control performance of Reinforcement learning is compared with a feedback linearization technique for the same task. The feedback linearization based controller is split into two loops, the internal loop for the attitude control running at higher rate as compared to the outer loop for the velocity control. Simulations are performed to compare both control techniques for attitude stabilization of the quadrotor. Simulation results show that the feedback linearization controller is able to stabilize attitude from $30^{\circ}$ initial angles within 0.6 seconds. Whereas the reinforcement learning based controller is able to stabilize the attitude in about one second with smaller overshoots as compared to the feedback linearization based controller.

Aggressive maneuvering of a quadrotor is achieved by Purvin et al. [95] using a 
reinforcement learning technique. The attitude of the quadrotor is maintained by a PD controller that is switched off while the iterative learning algorithm performs and switched back on when the maneuver is completed. Experimental results demonstrate a real-time performance of the controller being able to drive the system from one state to another.

A novel nonlinear neural networks and output feedback control technique is used by Dierks and Jagannathan [96]. The proposed control technique is developed to ensure hovering as well as tracking time varying trajectories while operating away from the hovering point. The neural network is allowed to learn online the complete dynamics of a quadrotor including nonlinear aerodynamic friction and blade flapping effects. A neural network based observer is used for angular velocity estimation and an output feedback control law is devised. Lyapunov theory is used to show that all the states and errors are semi-globally uniformly ultimately bounded in the presence of bounded disturbances. The flight control of a quadrotor is simulated in the presence of unmodeled dynamics and bounded disturbances. The maximum observer position error is found to be $0.03 \mathrm{~m}$ which increases with the introduction of disturbances and decreases by compensation made by the observer. To assess the proposed controller, a state feedback PID controller is implemented for the same task and its gains are carefully tuned. An acceptable tracking performance is achieved by the state feedback PID controller after significant gain tuning in the presence of introduced disturbances. The difference between two controllers is in the magnitude of control effort where PID based controller manages to achieve similar tracking performance with rotational torque control effort 4 to 5 times greater than that produced by the neural network based controller. The online learning advantage offered by neural networks is visible with this comparison where high PID gains are used to overcome neglected dynamics.

In a recent research by Efe [97], neural network based finite impulse response type approximator is used to imitate an analog $\mathrm{PI}^{\lambda} \mathrm{D}^{\mu}$ controller. The proposed 
neural network based control technique is used for the attitude stabilization of a quadrotor and compared with an analog $\mathrm{PI}^{\lambda} \mathrm{D}^{\mu}$ controller implemented through the use of the Crone approximation. To demonstrate the disturbance rejection by the proposed controller, the angular speeds of the quadrotor are perturbed additively to simulate wind conditions. Simulations are performed to evaluate the performance of both controllers and the results show a good attitude stabilization achieved by both controllers. It is observed that the errors caused by both controllers are almost indistinguishable in every axis of Cartesian space, which demonstrates the ability of the neural network based approximator as a substitute for analog $\mathrm{PI}^{\lambda} \mathrm{D}^{\mu}$ controller and the ease of its implementation via coefficient prediction.

Das et al. [98] use neural networks for the estimation of aerodynamic forces and moments acting on the quadrotor combined with an adaptive backstepping controller. A Lagrangian form of dynamics is used in the simulation to test the flight controller in the presence of introduced nonlinear terms representing aerodynamic forces and moments. The proposed controller demonstrates robust performance by explicitly dealing with the unmodeled state-dependent disturbances without any prior knowledge of such. The simulation results are compared against another backstepping approach [99]. The position of the quadrotor is stabilized within 10 seconds with a maximum error of $10 \mathrm{~cm}$. Attitude stabilization is also achieved within 3 seconds. The neural networks and backstepping control technique is able to achieve desired stabilization while effectively compensating for the unknown nonlinearities. 
Literature Review

\subsection{UAV Flight Performance in Wind Gust Con- ditions}

Despite design advantages, such as a VTOL hovering aircraft capable of various difficult maneuvers, a quadrotor is prone to disturbances that exist in outdoor environments. Disturbances of greater magnitude are primarily aerodynamic, such as wind gust conditions, and weather effects such as rain and snow. Disturbances of lower magnitudes include electronic disturbances such as signal interferences, and sensor noise. In realistic outdoor environment settings these disturbances are ever present. The low altitude flight regime places a quadrotor in a region referred to as the Urban Boundary Layer (UBL), in which turbulent wind gust conditions are prevalent. Therefore, as the size of the UAV is reduced (i.e MAV), combined with a low altitude flight regime, the winds become a greater percentage of a MAV's own speed, thus significantly affecting the flight performance of a MAV. Complicating this situation is the presence of urban structures such as buildings, trees, and canyons. In the vicinity of structures such as tall vertical buildings, there exist recirculation regions and vortices shedding downstream. In the wake of buildings the vortical flows present large variations in the wind velocity directions in a short distance. Autonomous flight of a Micro Aerial Vehicle (MAV) in such an environment is a challenging task which requires a clear understanding of existing wind fields and their effects on the MAV's flight performance. Recently, some research has emerged with results showing the effects of wind gust on the flight performance of MAVs and UAVs.

Orr et al. [100], at the Air Force Research Laboratory (AFRL), investigate the behaviour of a fixed-wing MAV flying through an urban setting. First, a replica of the actual flight test location, an urban setting, is constructed in MultiUAV 2.0 simulation environment. Wind data gathered from experiments and CFD simulations for the complex urban flows are modeled and integrated into the MultiUAV 2.0. The 
6DOF MAV model is also incorporated into the simulation environment. A waypoint sequencing routine is implemented and tested in the simulation environment, with and without wind conditions. The simulation results show that the MAV is able to perform designated waypoint sequencing while remaining within the specified tolerances. However, in the presence of a $4.6 \mathrm{~m} / \mathrm{s}$ constant wind speed, the MAV fails to perform the same task and is unable to reach the endpoint. The results emphasize the effects of a light breeze of $4.6 \mathrm{~m} / \mathrm{s}$ on a MAV, and the need of a robust flight control technique which can deal with adverse wind conditions.

In another relevant research, Galway et al. [101], [102], document the urban wind gust effects on the flight performance of an Aerosonde UAV and a Yamaha RC helicopter. The flight of the Aerosonde UAV over specified urban settings is simulated using a different methodology than that proposed by Orr et al. Wind fields around individual structures are evaluated beforehand and stored in a Wind Simulation Database (WSD). A selection algorithm is used to retrieve the wind fields local to the UAV, depending on its position within the selected urban setting comprised of several individual structures. This technique makes it possible to simulate wind fields in large urban areas without demanding higher computational loads. The results of the Aerosonde UAV flight through the wake of a building are acquired with different background wind speeds. It is demonstrated that even a $3.89 \mathrm{~m} / \mathrm{s}$ light wind condition causes a slight change in the heading of the UAV, which is increased significantly for a $13.89 \mathrm{~m} / \mathrm{s}$ wind condition. Yamaha RC helicopter flights are also simulated in a similar urban setting. First, a forward flight test is conducted to test the effects of building wakes on the flight performance of the helicopter flying through the wake with a background wind of $4.15 \mathrm{~m} / \mathrm{s}$. The heading of the helicopter starts oscillating with deviations as large as $8^{\circ}$. Similar results are obtained when the helicopter is allowed to fly within a close proximity to a wake boundary. In another test, a slow ascending flight within the wake of a building is conducted with the same background 
wind conditions. The position of the helicopter deviates slightly from a straight ascending path at the height of $70 \mathrm{~m}$, and a large deviation is observed at the height of $120 \mathrm{~m}$. This result demonstrates the effect of a recirculation zone that exists in the wake of a building, and identifies regions in the wake field that could pose a significant hazard for small size UAVs.

One of the most obvious and critical application areas where oceanic and atmospheric disturbances can pose a lethal danger is shipboard operations. UAVs taking off, flying in close vicinity to, or landing on a ship face significant disturbances, especially in the ship's wake.

Crump and Bil [103] investigate the launch of a fixed-wing UAV from a ship in extreme wind conditions. An atmospheric model based on steady state wind combined with Dryden turbulence is employed, where wind gust and windshear are modelled as effects of ship's airwake and ocean surface. The controller used for the autonomous launching is a $\mathcal{H}_{\infty}$ linear robust control for nominal launch conditions, combined with a trajectory optimization and inverse system identification method to deal with high headwind or tailwind disturbances. Several simulations are conducted to evaluate the performance of the proposed control method in the presence of tailwind, headwind and crosswind respectively. The simulation results for $10 \mathrm{~m} / \mathrm{s}$ tailwind reveal that despite successful launch, the effects of $10 \mathrm{~m} / \mathrm{s}$ tailwind can cause a growing error on the altitude. The 5 second flight data of the launch shows a $6 \mathrm{~m}$ error at 3 second time mark between the optimal and the actual trajectory, which grows to $10 \mathrm{~m}$ at the 5 second time mark.

The scenario of launching UAVs from ships is quite different from landing one on a ship by virtue of the speed of UAV in comparison with the wind speeds. During a launch the UAV is propelled at higher speed for a proper take-off, therefore accelerating through the turbulent environment near the ship, into steady air. On the contrary, during landing procedures a UAV is required to decelerate which makes it 
susceptible to the turbulence present. Wong et al. [104] study the landing accuracy of a fixed wing Aerosonde UAV on a moving frigate. The simulations are conducted on a hardware-in-the-loop setup where a Micropilot MP2028 autopilot is connected with a computer running a $6 \mathrm{DOF}$ simulation model of the UAV and a $3 \mathrm{DOF}$ model of the frigate using Matlab Simulink. The airwake data is generated offline in form of a precompiled database using PowerFLOW CFD analysis software running a Very Large Eddy Simulation (VLES) technique for a freestream input of 26 knots. Simulation results are provided, investigating the effects of various parameters, including frigate course, frigate speed, UAV airspeed, and target location, on the autopilot's ability to guide the UAV to the centre of a hypothetical 6x6 m capture device. Notably, simulations conducted in the absence of the modelled deck motion and airwake demonstrate an intrinsic miss distance of $1.5 \pm 0.9 \mathrm{~m}$. With the deck motion and airwake effects present, the average miss distance results for the lateral direction are $+3.03 \pm 5 \mathrm{~m}$, and $+1.11 \pm 2 \mathrm{~m}$ in the height direction. In order to ensure a $99.7 \%$ probability of recovery, the need for an unrealistic $21 \times 14 \mathrm{~m}$ capture device is postulated.

Shipboard operations involve both fixed-wing aircraft as well as rotary-wing vehicles such as a helicopter. The nonlinear dynamics of a helicopter are compounded with the ship's airwake turbulence in scenarios of approaching and hovering near the ship. Horn et al. [105] detail a model following controller integrated with an airwake compensator for aiding pilots during ship board operations. Flight simulations are carried out using a UH-60A Black Hawk helicopter model, approaching and hovering over the deck of a ship in a 30 degrees, 30 knot wind over deck scenario. Spectral filters incorporated into the model are used for replicating the power spectral density of turbulence in the ship's airwake. Simulation data demonstrate a successful reduction in attitude angle fluctuations with the airwake compensator, turned on, particularly on the roll axis, where \pm 10 degree approximate variations in attitude are decreased to within \pm 3 degrees. Again, the aim of this study is to reduce pilot work load by 
enhancing attitude stabilization during the period when the helicopter is in turbulent wind conditions, rather than developing a fully autonomous flight control.

Sydney et al. [106] also simulate an autonomous ship landing, this time of a quadrotor UAV. A feedback linearization controller is employed, including aerodynamic effects in the form of a recursive Bayesian estimate of the prevailing wind, based on assumed ground velocity and air velocity measurements. Compared with previously mentioned shipboard studies, here the airwake is represented by a simplified generated flowfield based on a modest $0.5 \mathrm{~m} / \mathrm{s}$ prevailing wind, supplemented with frozen Dryden turbulence. Simulation results show the quadrotor travelling between two points, $2 \mathrm{~m}$ apart in both the horizontal and vertical directions, to reach a specified landing location. The x position deviates up $40 \mathrm{~cm}$ from the desired coordinates, before settling within $25 \mathrm{~cm}$ of the target position.

Another approach in dealing with wind disturbances is to develop online estimation techniques which can effectively predict the wind disturbance applied on the UAV. Hancer et al. [107] include wind effects in the form of Dryden wind turbulence in the dynamic model of a tilt-wing quadrotor. A PID position control is implemented along with a disturbance observer based on the actuator input. Although experimentally tested, only simulation results are presented for the attitude and position hold control performance. A trajectory tracking simulation shows the quadrotor remaining within $1 \mathrm{~m}$ of a reference path in up to a $1 \mathrm{~N}$ force wind disturbance.

Waslander and Wang [108] present an estimation algorithm for the wind velocity experienced by a quadrotor UAV, also based on the Dryden wind gust model. The estimated wind velocity from accelerometer data is used to augment a position controller with a wind compensation term, following the assumption that the current estimated wind will not significantly change until the next measurement and can be subsequently employed as the assumed disturbance. Simulation results are provided, 
whereby the wind compensator improves the hover performance of a PID,DD controller in winds ranging approximately $\pm 3 \mathrm{~m} / \mathrm{s}$ reducing the errors from $\pm 0.15 \mathrm{~m}$ to the $\pm 0.1 \mathrm{~m}$ range. However, as stated in the paper, the presented research is intended as an initial proof of concept for this method, with further examination of accelerometer data and quadrotor dynamics required.

Alexis et al. [109] use a switching model predictive control for the attitude and altitude control of a quadrotor in the presence of wind disturbances. The wind disturbance is modeled as a random Gaussian noise with zero mean and unity covariance matrix. The white noise unmeasured disturbances are modeled as an integrator output. The controller is based on Piecewise Affine models of the attitude and altitude dynamics of the quadrotor. The switching logic relies on the rate of rotation angles and estimated vertical velocity acquired using extended Kalman filter on the measured vertical acceleration. The a-posteriori altitude measurements are acquired by a sonar and combined with vertical acceleration and velocity data using the EKF resulting in efficient altitude control. Experimental results are evaluated under wind disturbance generated using a wind turbine producing continuous wind on the scale of 3 Beaufort (3.4-5.4 m/s), measured with a rotary vane anemometer. The additive wind disturbance bounds are measured as $0.15 \mathrm{rad} / \mathrm{s}$ for attitude angular rates and $0.1 \mathrm{~m} / \mathrm{s}$ for the vertical axis. The switching model predictive controllers for attitude stabilization are able to maintain the attitude angles within $0.25 \mathrm{rad}\left(14.3^{\circ}\right)$ range. The altitude controller maintains position hold with an error margin of $\pm 25 \mathrm{~cm}$. The wind disturbance is measured as $1.48 \mathrm{~m} / \mathrm{s}$ for the $\mathrm{x}$-axis, $2.69 \mathrm{~m} / \mathrm{s}$ for the $\mathrm{y}$-axis, and $1.56 \mathrm{~m} / \mathrm{s}$ for the z-axis.

Shortly afterwards, this existing design is extended in another publication by Alexis et al. [110], a full three axis position control is presented along with the attitude control, where the added lateral position is estimated by fusing optical flow sensor readings with IMU based accelerations. The experimental results show a lateral 
position hold within a $20 \mathrm{~cm}$ radius in the presence of $4.96 \mathrm{~m} / \mathrm{s}, 1.31 \mathrm{~m} / \mathrm{s}$, and 1.22 $\mathrm{m} / \mathrm{s}$ wind speeds for the $\mathrm{x}, \mathrm{y}$ and $\mathrm{z}$-axis respectively. However, the $\mathrm{x}$-axis position converges at best within $10 \mathrm{~cm}$ of the desired coordinates once the fan based wind gusts, which are comparable to freestream conditions, are introduced. As the authors state, the presence of turbulence would present more complex aerodynamic effects to challenge the controller.

\subsection{Summary}

In the light of the literature presented in earlier sections, it is evident that a substantial body of work exists investigating the attitude stabilization of a quadrotor. A simple control technique such as PID [31] has been shown to be able to stabilize the quadrotor with $\pm 1^{\circ}$ precision in a hover state within an indoor environment. However, PID control is not sufficient to deal with large disturbances on its attitude axes. It is also shown that the attitude stabilization can be further improved with the addition of a PD control loop based on BLDC motor armature current feedback [36]. Similarly, a backstepping control alone, is found to be insufficient for attitude stabilization, while the integral-backstepping control scheme [60], shows successful attitude stabilization, even in the presence of disturbances such as constant wind and change in mass and inertia parameters. It is also found that the sliding mode controller [31], due to its switching nature, is not successful in achieving autonomous hover. Similar performance is reported with the LQR control technique [70]. However, a Constrained Finite Time Optimal (CFTO) control [78], demonstrates successful attitude stabilization in the presence of wind disturbances. Subsequently, model predictive control technique [110] is used to stabilize the position of a quadrotor when subjected to wind flow from a ducted fan. $\mathcal{H}_{\infty}$ based robust control [80], is able to stabilize the 
quadrotor and showed $40 \%$ to $70 \%$ performance increase when compared with a backstepping controller. Adaptive control techniques, mostly tested in simulations, are generally successful. However, they show a common disadvantage of higher settling times, thereby presenting a need to devise faster adaptation control laws. Among the intelligent control techniques, fuzzy logic based controllers suitable for dealing with disturbances are only tested in simulations and found to be slower, as compared to self-ruled fuzzy controllers [92]. Neural network based control, also tested in simulations, shows some advantages over state feedback PID and backstepping control techniques. Reinforcement learning-based controllers also demonstrate some promising performance, which is backed by experimental results [27]. Furthermore, onboard vision based adaptive control techniques such as [111], and visual servo techniques such as [112], [113], [114], provide the advantage of improved attitude and position estimates, while eliminating noise and drift issues typically associated with IMU and GPS.

It can be observed that significant experimental results are available for techniques such as PID, backstepping, feedback linearization, sliding mode and LQR. These experimental results include mostly indoor flights and a very few cases of limited outdoor flights. On the other hand, control techniques such as adaptive, fuzzy, $\mathcal{H}_{\infty}$, and neural networks are mostly presented with simulation results.

Wind disturbances are considered while testing flight controls of various UAVs in shipboard applications [103], [104], [105]. In simulation, these wind conditions are modeled either using post-processed CFD analysis-based wind velocities, or most commonly, through a Dryden turbulence model combined with steady state winds. In general, positional errors in excess of $3 \mathrm{~m}$ are reported, for UAVs which are considerably larger than average-sized quadrotors. Only in a single case, Sydney [106] used a quadrotor for a shipboard landing simulation. However, the wind disturbances mentioned are small enough not to produce any significant impact on flight performance. 
Further work in the area of wind perturbations has focused on the online identification of these disturbances through estimation techniques [107], [108]. To date, results in support of these efforts have been presented exclusively with simulation data, with real-world testing still required.

In comparison with the comprehensive attitude control literature, the outdoor position control of a quadrotor with better than a typical GPS accuracy using onboard sensing remains very much an open field. Therefore, an analysis on the effects of various wind-based disturbances on the flight performance of a quadrotor is required. Also, it is necessary to investigate how different position control techniques will perform under the same disturbances. Hence, this thesis is concerned with filling in the gap in current quadrotor research. 


\section{Chapter 3}

\section{Simulation Methodology}

This chapter details the systematic methodology employed in order to test potentially successful control techniques under realistic wind conditions anticipated for UAVs in numerous proposed real-world scenarios. For the performance of different controllers to be comparatively analyzed in a meaningful manner, consistent and reproducible simulation parameters are defined, in terms of the unmanned aircraft employed, the representational urban environment with its corresponding wind gust profile, and the specific sequence and coordinates of the autonomous flights performed.

A quadrotor prototype called TARA is designed and developed to serve as the UAV testbed for this research. The particulars of the TARA quadrotor's physical structure, actuators, sensors, communication capabilities and software are provided in Appendix-A. To evaluate the performance of candidate control methodologies, a customized flight simulator is configured with TARA's mathematical model and physical parameters. A single-building configuration is selected as the experimental landscape and implemented within the simulator. The simulator's predictive capability is enhanced by the inclusion of urban wind velocity data obtained from LES-based computational fluid dynamics of the airflow around the defined urban structure. The various flight scenario test cases and the evaluation criteria employed in assessing comparative results are presented in detail. 


\subsection{Flight Simulation with Urban Wind Model}

This section details the mathematical model of a quadrotor, the experimentally identified parameters of the actual UAV prototype, and the building blocks of the flight simulator which is used to simulate TARA's flight in urban wind gust conditions.

\subsubsection{Mathematical Modeling of TARA}

In developing the mathematical model of TARA some reasonable simplifying assumptions are made. The first of these is that TARA's frame is held to be symmetrical. Therefore all cross moments of inertia are zero, resulting in a diagonal moment of inertia matrix. It is also presupposed that the rigid body assumption holds for the quadrotor structure. Finally, the COG of the quadrotor is taken to coincide with the center of the rigid frame.
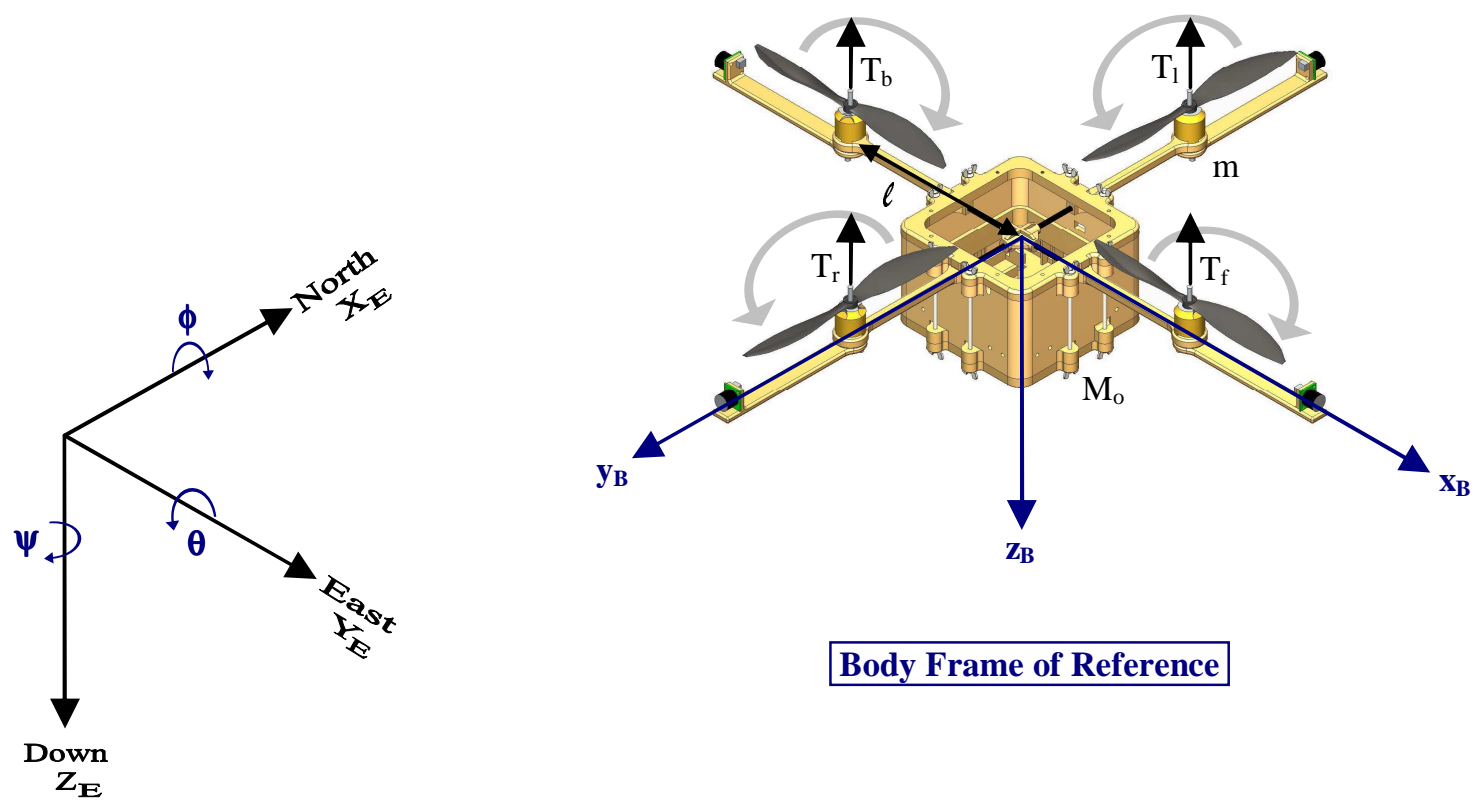

Body Frame of Reference

Earth Frame of Reference

Figure 1: Earth and body reference frames.

During the flights TARA is required to maintain attitude angles below $90^{\circ}$, thus 
avoiding the gimbal lock condition. Therefore, the Euler angle parameterization, ( roll $=\phi$, pitch $=\theta$, and yaw $=\psi$ ), is used for defining the orientation of TARA, and is considered to be sufficient for this application. Two reference frames, as shown in Figure 1, are used to define the quadrotor's attitude and its position in space. The body fixed frame $B$ is placed such that its origin is located at the center of TARA's frame. The x-axis $\overrightarrow{x_{B}}$ is aligned with the front motor arm, and the $\mathrm{y}$-axis $\overrightarrow{y_{B}}$ is aligned with the right side motor arm. The z-axis $\overrightarrow{z_{B}}$ is perpendicular to the $\mathrm{x}-\mathrm{y}$ plane, pointing towards the bottom of the chassis. The earth fixed frame of reference $E$ uses the NED convention whereby the x-axis $\overrightarrow{X_{E}}$ points towards the North, the y-axis $\overrightarrow{Y_{E}}$ points towards the East, and the z-axis $\overrightarrow{Z_{E}}$ points towards the center of the earth.

Starting with coincident earth and body frames, the Euler angles corresponding to a particular orientation of the quadrotor can be defined through a sequence of rotations:

- A yaw rotation about the body frame Z-axis by an angle $\psi$, described by matrix $R_{Z}(\psi)$

- A pitch rotation about the resulting intermediate body frame Y-axis by an angle $\theta$, described by matrix $R_{Y}(\theta)$

- A roll rotation about the subsequent intermediate body frame X-axis by an angle $\phi$, described by matrix $R_{X}(\phi)$

Hence, the complete rotation matrix, $R$, is given by:

$$
R=R_{Z}(\psi) R_{Y}(\theta) R_{X}(\phi)
$$


where

$$
R=\left[\begin{array}{ccc}
\cos \psi & \sin \psi & 0 \\
-\sin \psi & \cos \psi & 0 \\
0 & 0 & 1
\end{array}\right]\left[\begin{array}{ccc}
\cos \theta & 0 & -\sin \theta \\
0 & 1 & 0 \\
\sin \theta & 0 & \cos \theta
\end{array}\right]\left[\begin{array}{ccc}
1 & 0 & 0 \\
0 & \cos \phi & \sin \phi \\
0 & -\sin \phi & \cos \phi
\end{array}\right]
$$

and the resulting rotation matrix is:

$$
R=\left[\begin{array}{c:c:c}
\cos \theta \cos \psi & \sin \phi \sin \theta \cos \psi-\cos \phi \sin \psi & \cos \phi \sin \theta \cos \psi+\sin \phi \sin \psi \\
\cos \theta \sin \psi & \sin \phi \sin \theta \sin \psi+\cos \phi \cos \psi & \cos \phi \sin \theta \sin \psi-\sin \phi \cos \psi \\
& \sin \phi \cos \theta & \cos \phi \cos \theta
\end{array}\right]
$$

The numerical vector of Euler rates is:

$$
\left[\begin{array}{c}
\dot{\phi} \\
\dot{\theta} \\
\dot{\psi}
\end{array}\right]=\left[\begin{array}{ccc}
1 & \sin \phi \tan \theta & \cos \phi \tan \theta \\
0 & \cos \phi & -\sin \phi \\
0 & \sin \phi \sec \theta & \cos \phi \sec \theta
\end{array}\right]\left[\begin{array}{c}
p \\
q \\
r
\end{array}\right]
$$

where $\left[\begin{array}{lll}\dot{\phi} & \dot{\theta} & \dot{\psi}\end{array}\right]^{T}$ are the attitude angular rates of TARA in the earth fixed frame obtained by multiplying the body frame angular rates $\left[\begin{array}{ll}p & q\end{array}\right]^{T}$ with a rotation matrix. Similarly, TARA's velocity in the earth fixed frame can be evaluated by applying 
a transformation matrix $R$ such that:

$$
\left[\begin{array}{c}
\dot{X} \\
\dot{Y} \\
\dot{Z}
\end{array}\right]_{E}=R\left[\begin{array}{l}
u \\
v \\
w
\end{array}\right]_{B}
$$

where $\left[\begin{array}{lll}\dot{X} & \dot{Y} & \dot{Z}\end{array}\right]^{T}$ are the velocities of TARA in earth-fixed frame $E,\left[\begin{array}{ll}u & w\end{array}\right]^{T}$ are the body frame $B$ velocities and $R$ is the rotation matrix used for body frame to earth frame transformation (per Equation 15).

Considering a rigid body with forces and torques acting on the COG, the NewtonEuler formulation with respect to the quadrotor's body frame may be expressed as:

$$
\left[\begin{array}{c}
F_{B} \\
\tau_{B}
\end{array}\right]=\left[\begin{array}{cc}
M I_{3 \times 3} & 0 \\
0 & J I_{3 \times 3}
\end{array}\right]\left[\begin{array}{c}
\dot{V}_{B} \\
\dot{\omega}_{B}
\end{array}\right]+\left[\begin{array}{c}
\omega_{B} \times M V_{B} \\
\omega_{B} \times J \omega_{B}
\end{array}\right]
$$

where $M=M_{0}+4 m$ is the quadrotor's total mass, $m$ is the mass of a motor, $F_{B}=\left[\begin{array}{lll}f_{x} & f_{y} & f_{z}\end{array}\right]^{T}$ are the forces, and $\tau_{B}=\left[\begin{array}{lll}\tau_{\phi} & \tau_{\theta} & \tau_{\psi}\end{array}\right]^{T}$ are the torques applied on TARA's COG.

The forces acting on TARA include the aerodynamic drag, the lift generated by each rotor, and the weight. The moments are the torques generated in order to achieve the roll, pitch, and yaw movements. Among the forces to consider are those which are produced by the quadrotor itself. These quadrotor-generated forces included in the simulation model are:

Upward Force (Thrust): The total quadrotor thrust is the sum of thrust produced by each motor:

$$
T=T_{f}+T_{r}+T_{b}+T_{l}
$$


The upward force is therefore:

$$
-f_{z}=T
$$

Gravitational Force (Weight): The gravitational force acts on the COG of the quadrotor. This force is expressed as:

$$
G=\left[\begin{array}{c}
0 \\
0 \\
M g
\end{array}\right]_{E}
$$

where $g$ is the gravitational constant. In the body frame the weight can be written as:

$$
G=\left[\begin{array}{c}
-M g \sin \theta \\
M g \cos \theta \sin \phi \\
M g \cos \theta \cos \phi
\end{array}\right]_{B}
$$

Rolling Torque: Positive rolling torque is produced by increasing the left rotor thrust while decreasing the right rotor thrust:

$$
\tau_{\phi}=l\left(T_{l}-T_{r}\right)
$$

Pitching Torque: Positive pitching torque is produced by increasing the front rotor thrust while decreasing the back rotor thrust:

$$
\tau_{\theta}=l\left(T_{f}-T_{b}\right)
$$


Yawing Torque: The torque generated by an individual motor is calculated as the product of the motor torque constant $\left(K_{\tau}=0.02\right)$ for the RimFire 400 BLDC motor, and the thrust. The yawing torque is the resultant of all four individual torques generated due to the spinning rotors. The front and back rotors spin in the clockwise direction, while the left and right rotors spin in the counterclockwise direction. An imbalance between these two pairs results in a yawing torque causing the quadrotor to rotate about its z-axis:

$$
\begin{gathered}
\tau_{\psi}=\tau_{r}+\tau_{l}-\tau_{f}-\tau_{b} \\
\tau_{\psi}=K_{\tau}\left(T_{r}+T_{l}-T_{f}-T_{b}\right)
\end{gathered}
$$

Adding Wind Effects: The effect of wind on the quadrotor is implemented by adding the wind velocity components to the quadrotor's own velocities in the body frame:

$$
\left[\begin{array}{c}
W x \\
W y \\
W z
\end{array}\right]_{B}=R^{-1}\left[\begin{array}{c}
W n \\
W e \\
W d
\end{array}\right]_{E}+\left[\begin{array}{l}
u \\
v \\
w
\end{array}\right]_{B}
$$

where $[W n W e W d]^{T}$ are the (NED) wind velocities in the earth fixed frame transformed to body fixed frame using $R^{-1}$ and added with TARA's body frame velocities $\left[\begin{array}{lll}u & v & w\end{array}\right]^{T}$ to get resultant velocities $[W x W y W z]^{T}$ of TARA in the presence of wind.

Aerodynamic Drag on Fuselage: The fuselage comprises the most volume of the quadrotor's body. It contains the main payload including sensors, communication and control electronics along with the batteries. The fuselage, being a bluff body, is subjected to drag forces due to the environmental wind conditions $[W x W y W z]^{T}$, and air resistance when the quadrotor is in motion. The fuselage force is determined 
as the product of local dynamic pressure and the corresponding fuselage effective wet area. It is evaluated to be:

$$
F_{\text {aero }}=\left[\begin{array}{c}
F_{\text {aero } x} \\
F_{\text {aero } y} \\
F_{\text {aero } z}
\end{array}\right]=-\frac{1}{2} \rho\left[\begin{array}{l}
(W x)^{2} \cdot C_{d_{x}} \cdot S_{x} \frac{W x}{|W x|} \\
(W y)^{2} \cdot C_{d_{y}} \cdot S_{y} \frac{W y}{|W y|} \\
(W z)^{2} \cdot C_{d_{z}} \cdot S_{z} \frac{W z}{|W z|}
\end{array}\right]
$$

where $F_{\text {aero }}$ is the total aerodynamic drag force acting on the quadrotor's body, $[W x W y W z]^{T}$ are the body frame velocities in the presence of wind, and $\rho$ is the ambient air density. The drag coefficient of a cube $C_{d_{x}}=C_{d_{y}}=C_{d_{z}}=1.05$ at $R e>10^{4}$ is used for the fuselage body. The $S_{x}=S_{y}=0.0136 \mathrm{~m}^{2}$, and $S_{z}=0.0256 \mathrm{~m}^{2}$, are the fuselage effective wet areas normal to the $\mathrm{x}, \mathrm{y}$, and $\mathrm{z}$ axes respectively, are used in the simulation model.

\subsubsection{Equations of Motion}

Including the forces and torques acting on the system, the six-degree-of-freedom equations of motion (Equation 18) in the body fixed frame used for flight simulation become,

$$
\left[\begin{array}{c}
\dot{u} \\
\dot{v} \\
\dot{w}
\end{array}\right]=\left[\begin{array}{c}
r v-q w \\
p w-r u \\
q u-p v
\end{array}\right]+\left[\begin{array}{c}
-g \sin \theta \\
g \cos \theta \sin \phi \\
g \cos \theta \cos \phi
\end{array}\right]+\frac{1}{M}\left[\begin{array}{c}
0 \\
0 \\
-T
\end{array}\right]+\frac{1}{M}\left[\begin{array}{c}
F_{\text {aero }} \\
F_{\text {aero }} \\
F_{\text {aero }}
\end{array}\right]
$$




$$
\left[\begin{array}{c}
\dot{p} \\
\dot{q} \\
\dot{r}
\end{array}\right]=\left[\begin{array}{c}
\frac{j_{y}-j_{z}}{j_{x}} q r \\
\frac{j_{z}-j_{x}}{j_{y}} p r \\
\frac{j_{x}-j_{y}}{j_{z}} p q
\end{array}\right]+\left[\begin{array}{c}
\frac{1}{j_{x}} l\left(T_{l}-T_{r}\right) \\
\frac{1}{j_{y}} l\left(T_{f}-T_{b}\right) \\
\frac{1}{j_{z}}\left(\tau_{r}+\tau_{l}-\tau_{f}-\tau_{b}\right)
\end{array}\right]
$$

\subsubsection{Experimental Identification of Parameters}

The mathematical model of TARA incorporates parameters extracted experimentally from the real prototype, such as the moment of inertia (Equation 31) and the thrust model (Equation 32). Physical parameters such as mass, moment of inertia, and the thrust generated by the motor driven propellers are needed for accurately modeling the UAV. The mass of the quadrotor is measured to be $2.2 \mathrm{~kg}$, using a tabletop weight measure with an accuracy of $\pm 1 \mathrm{~g}$.

Moments of Inertia: The moments of inertia of TARA are obtained using the compound pendulum method. The $j_{z}$ value is taken from a CAD model which includes onboard components such as motors, batteries, the RF receiver and miscellaneous circuits. The inertia tensor is calculated to be a diagonal matrix due to the symmetry of the quadrotor:

$$
J=\left[\begin{array}{ccc}
0.0150 & 0 & 0 \\
0 & 0.0138 & 0 \\
0 & 0 & 0.0205
\end{array}\right] \mathrm{kg} \cdot \mathrm{m}^{2}
$$

Propulsion Model: Motor speeds are controlled using Electronic Speed Controller (ESC) modules which take a standard PWM signal as input and apply corresponding voltages to change the motor speed. Therefore, for the thrust model the PWM signal is taken as the input to the propulsion unit and its output is the thrust generated by 
the motor-driven propeller.

For measurement purposes the motor on one side of the centrally pivoted quadrotor is allowed to run, while the other end is placed on top of a weighing measure to record the thrust versus the PWM signal. The PWM signal required to drive the motor is generated using a 10-bit PWM channel available on the ATMega640 microcontroller, such that the range of integers 250-500 is mapped to produce a corresponding pulse width of 1-2 ms. The thrust obtained using the weighing measure is then converted into Newtons. This experiment is repeated to acquire the plots of all four individual propulsion units. Figure 2(a) shows the thrust versus PWM plots of each of the propulsion units, as well as their average. Curve fitting is then utilized,

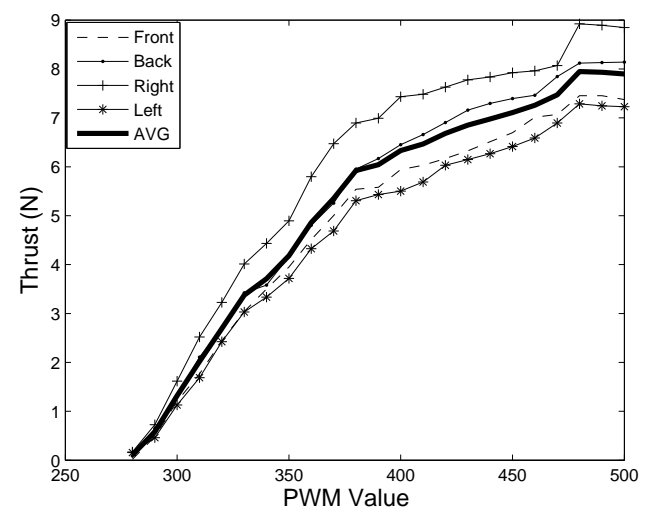

(a)

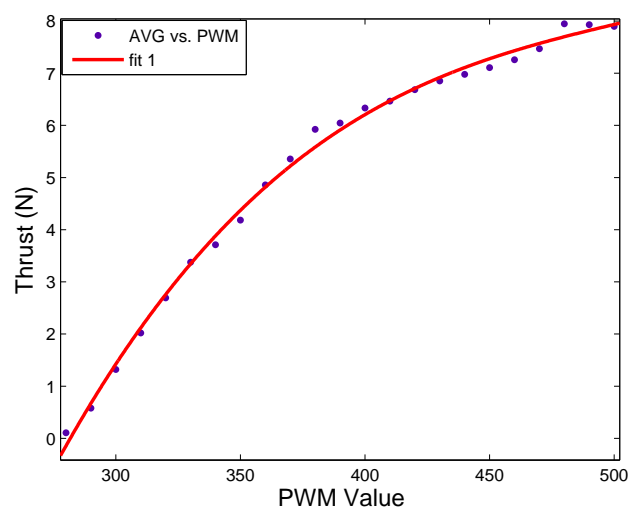

(b)

Figure 2: Thrust plotting of experimental data. (a) Thrust plots of all four propulsion units. (b) Curve fitting for averaged propulsion unit.

as shown in Figure 2(b), to model the averaged propulsion unit plot in the form of a 3rd order polynomial. Equation 32 is then implemented into the simulation model,

$$
T(p w m)=p 1 \times(p w m)^{3}+p 2 \times(p w m)^{2}+p 3 \times(p w m)+p 4
$$

where $\mathrm{p} 1=4.648 \mathrm{e}-007, \mathrm{p} 2=-0.0007105, \mathrm{p} 3=0.3732$, and $\mathrm{p} 4=-59.14$, are the coefficients for the averaged propulsion unit. 
Motor Time Constant: The time constant of the BLDC motors is also found experimentally. A microphone is placed close to the propeller in order to register the compression pulse generated by the passing wing. With this experimental setup the RPM of the propeller is measured over a range of PWM input commands. Using the recorded step response data the time constant is determined. For these motors an average value is found of $t_{m}=0.26$ seconds. The resulting propulsion model of a single motor implemented in the simulator is shown in Figure 5.

\subsubsection{TARA Flight Simulator}

To evaluate and test potential autonomous flight control techniques, a flight simulator is developed which incorporates the mathematical model of TARA and its experimentally-identified parameters. A fixed-step ode3 (Bogacki-Shampine) solver is used to solve the ordinary differential equations representing the model. A modular approach is adopted in the design of the simulator, such that in order to test a new flight control technique, only the flight controller module needs to be replaced. The simulator also includes an urban wind model which is responsible for producing wind gust environments. Figure 3 illustrates an overview of the flight simulator implemented as a Matlab Simulink model, showing the essential components such as the TARA UAV, Flight Controller, Urban Wind Effects, and user inputs in block form. Each of these blocks are presented in detail to explain the functioning of the flight simulator.

TARA UAV Block: The derived equations of motion are implemented as a Simulink model block named TARA UAV for simulation purposes. As shown in Figure 4, the internal feedback input states to this model are the four vectors representing the position $\left[\begin{array}{lll}X & Y & Z\end{array}\right]^{T}$, velocity $\left[\begin{array}{lll}u & v & w\end{array}\right]^{T}$, rotational angles $\left[\begin{array}{ll}\phi, & \theta, \psi\end{array}\right]^{T}$, and angular velocity $\left[\begin{array}{lll}p & q & r\end{array}\right]^{T}$ of the UAV. The external inputs include four inputs 
TARA Flight Simulator

Revision Date: 1 September 2014
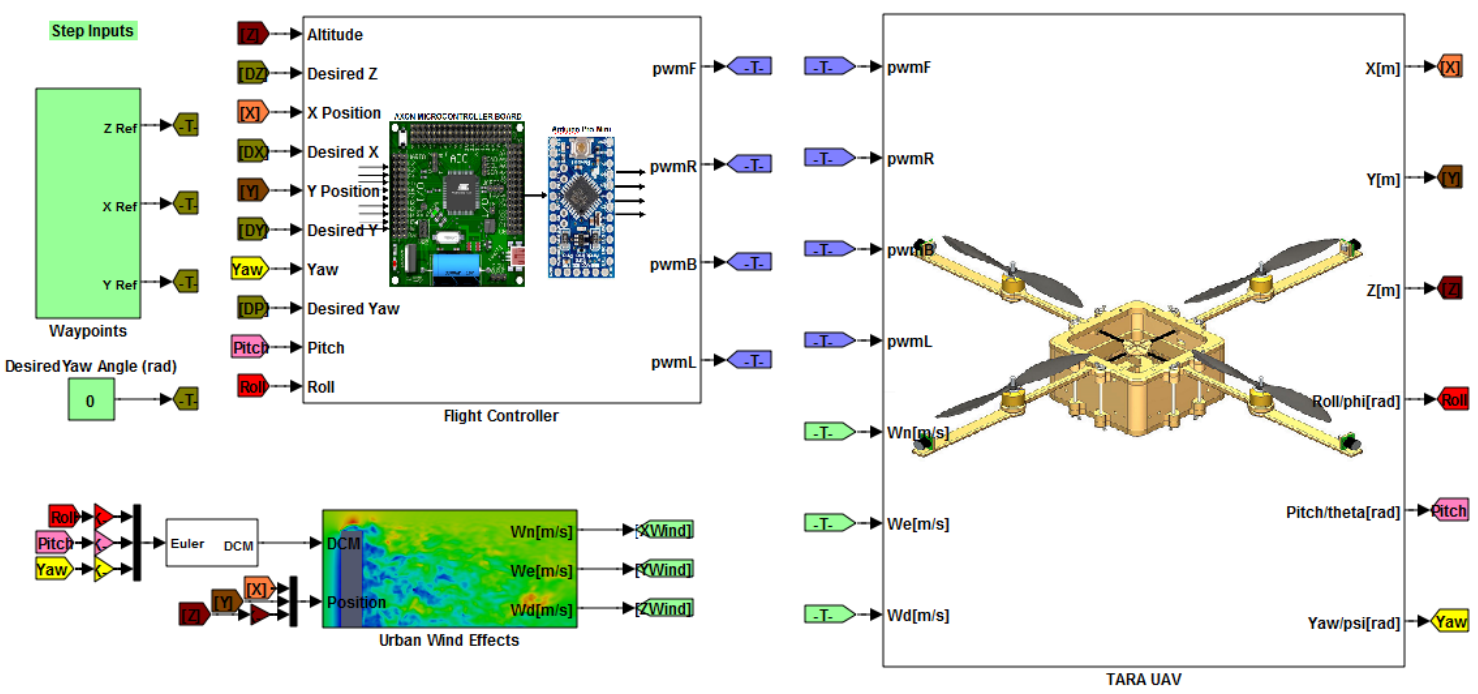

Figure 3: Simulink model of TARA flight simulator.

for the thrust $\left[\begin{array}{llll}T_{f} & T_{r} & T_{b} & T_{l}\end{array}\right]^{T}$, from each of the individual propulsion units. Since the simulations will also be considering turbulent wind, three wind velocity inputs $[W n W e W d]^{T}$ for the winds in NED earth frame coordinates are included.

The outputs of the TARA dynamics block are the derivatives of state vectors

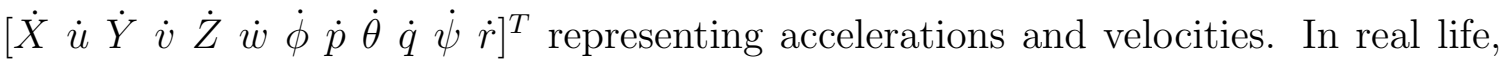
accelerometers and gyroscopes are used to measure the body accelerations $\left[\begin{array}{lll}\dot{u} & \dot{v} & \dot{w}\end{array}\right]^{T}$ and angular rates $\left[\begin{array}{lll}p & q & r\end{array}\right]^{T}$ of a robot, which include sensor bias and noise errors. Integrating such measurements provides the position and attitude of the robot, but with growing errors. To simulate the inertial sensor errors, the twelve aforementioned outputs are first processed through a three-axis inertial measurement unit (IMU) block, taken from the Aerospace Blockset library. The purpose of this IMU block is to simulate the second-order dynamics of a three-axis gyroscope and a three-axis accelerometer. Noise is added to the processed signals, and the power of noise is set to match that of the MEMS based accelerometers $\left(50 \frac{\mu g}{\sqrt{H z}}\right)$ and gyroscopes $\left(0.014 \frac{\mathrm{deg} / \mathrm{s}}{\sqrt{H z}}\right)$ 


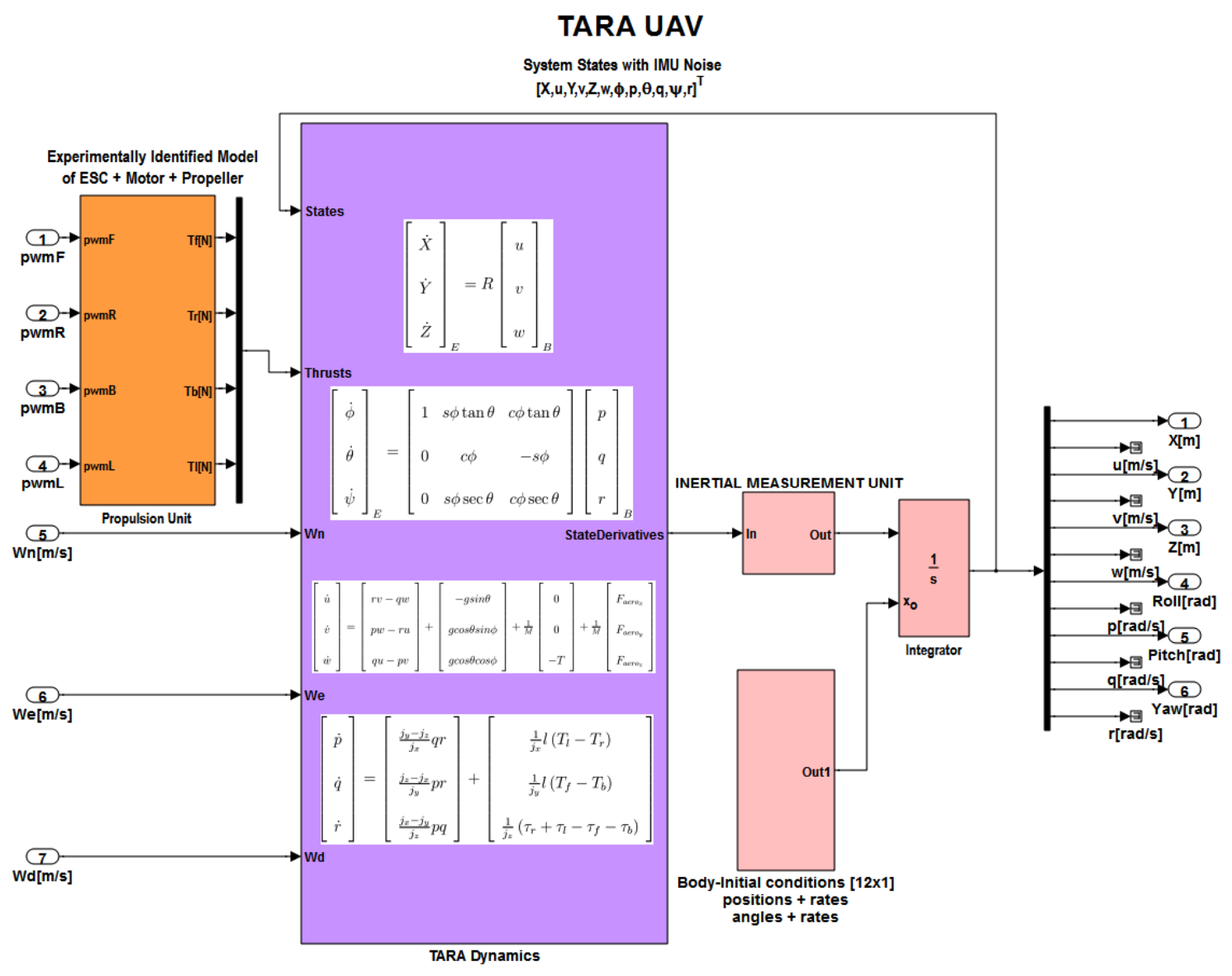

Figure 4: TARA UAV block.

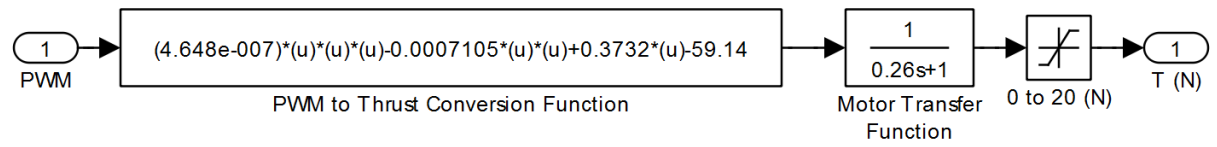

Figure 5: Propulsion model for a single motor. 
used on-board. The output of the IMU block is integrated with the desired initial conditions to produce the appropriate position, velocities, attitude angles, and angular velocities of the quadrotor. These are fed back into the quadrotor UAV model as inputs to complete a closed loop system.

Propulsion Model Block: The propulsion unit model provides the four thrust inputs to the quadrotor UAV model. The inputs to the propulsion model are four integer PWM values describing the desired speed of the motors, thereby using Equation 32 the equivalent thrusts are produced.

Urban Wind Effects Block: The urban wind model running its selection algorithm is responsible for querying appropriate wind velocity vectors from a precompiled wind database, based on TARA's position coordinates at a specific time step. The obtained wind velocity components are applied to the COG of the quadrotor as per Equation 27 and 28. As the wind disturbances are applied to a single point on the body, therefore no angular moments are produced due to the wind. In the case of a larger aircraft, the wind disturbances can be applied to three or more points on the body, resulting in effective angular rates. However, TARA's size compared to the velocity gradients from the urban windfield is small and thus variations in wind velocities over this length of TARA are neglected.

Flight Controller Block: The flight controller block consists of three stages: preprocessing, flight control, and post-processing. The first stage of pre-processing involves calculating the position and yaw errors. These errors are then passed to the flight control stage where the errors are saturated to satisfy the controller's input requirements. As shown in Figure 6, the flight control is implemented in a cascade formation representing the functioning of the Arduino and the Axon as the attitude and position controllers, respectively. The modular design of this stage allows for the 
efficient implementation of different control techniques which can be easily swapped in and out of the system as desired. The control outputs are then sent to the last stage of post-processing, shown in Figure 7, where altitude, pitch, roll, and yaw control outputs are combined and converted to produce the four motor speed PWM values.

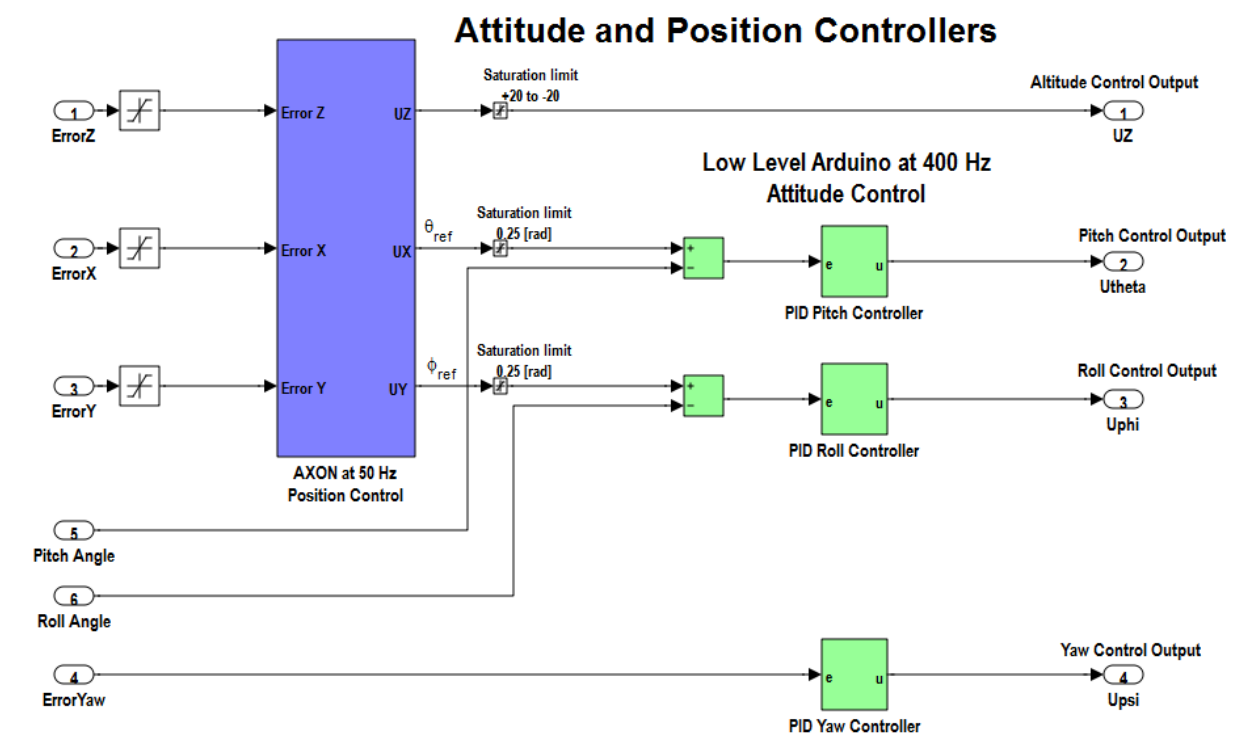

Figure 6: The control stage in the flight controller block.

\subsection{Large Eddy Simulation Based Wind Model}

Urban wind modeling is gaining increasing interest due to developments in many areas. Consider, for example, simulating the flight of a UAV in urban environments, which would require including realistic wind turbulence acting on the vehicle while flying in close proximity to buildings. Modern flight simulation environments do provide wind turbulence models, such as the Dryden and Von Karman models available in Matlab's Aerospace Blockset. However, these models are generalized and do not account for the presence of urban structures, where airflow around such structures is classifiable with significant degrees of circulation and sheer. Furthermore, both 


\section{Post-Processing Block}

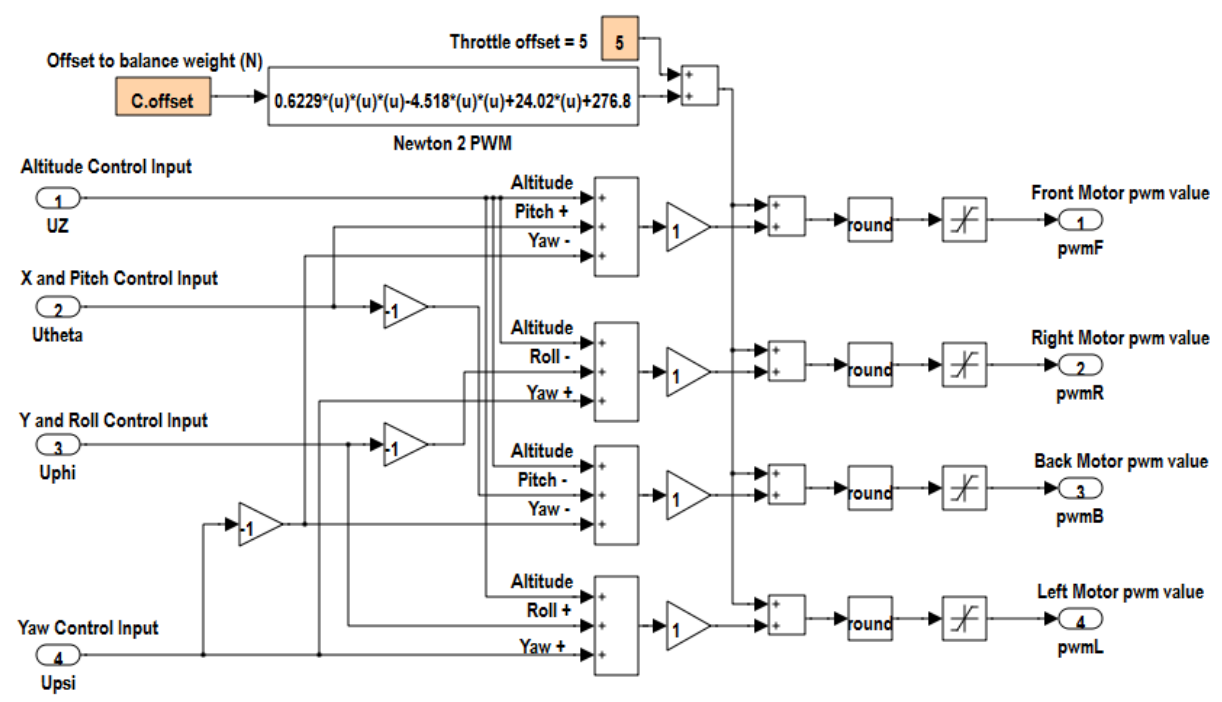

Figure 7: The post-processing stage in the flight controller block.

models require motion through the gusts to produce varying turbulence making them inappropriate for use in hover flight.

A substantial amount of literature exists on the subject of flow around individual urban structures. For instance, a study [115] is conducted to document the turbulent wind flows around the buildings and urban canyons in order to observe pollutant dispersion in urban environments. Interestingly, there is an increasing trend in the use of Computational Fluid Dynamics (CFD) for analyzing wind flows past buildings in many recent studies. For example, in another study [116], pollutant dispersion in an urban environment is analyzed using CFD and the advantages and disadvantages of CFD are also discussed. In Tokyo, Japan, Murakami et al. [117] analyzed the various physical processes associated with wind climates around humans and presented CFD analyses of outdoor climates ranging from human to urban in scale. Similarly, [118] [119] and [120] use CFD to simulate flows around various building structures, such as airport terminal buildings and pedestrian walkways. The sensitivity of urban flow characteristics to the selection of turbulence model is documented in [121] and [122]. 
Parameterization of flow around simple building structures is investigated by [123] [124] and [125].

Consider a simple example, where CFD is used to generate flows around a cylinder [126] as shown in Figure 8. It can be seen in Figure 8(b) that at lower Reynolds numbers, such as 10 to 20, a pair of stable and symmetric vortices will form behind the cylinder. But as the Reynolds number increases, as shown in Figure 8(c), the vortices forming behind the cylinder shed downstream in an alternating pattern known as a Von Karman vortex street.

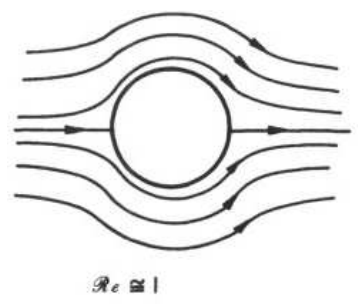

(a)

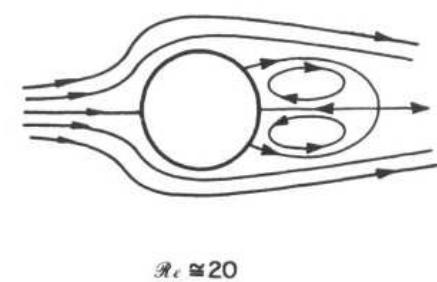

(b)

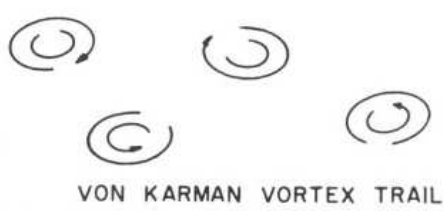

(c)

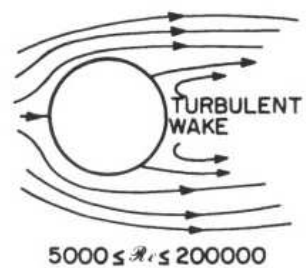

(d)

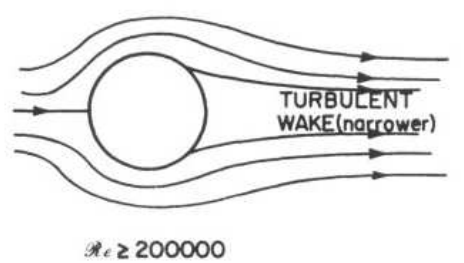

(e)

Figure 8: Flow past a circular cylinder with different flow regimes [126].

The hydrogen bubble visualization technique is used and compared with CFD simulation results to analyze laminar flow past different bluff bodies including a square cube [127]. Laminar steady and unsteady flows are investigated and photographs 
taken for the real flow visualization, as shown in Figure 9. The CFD numerical modeling (shown inset Figure 9) is found to be markedly similar to the visualized real flow.

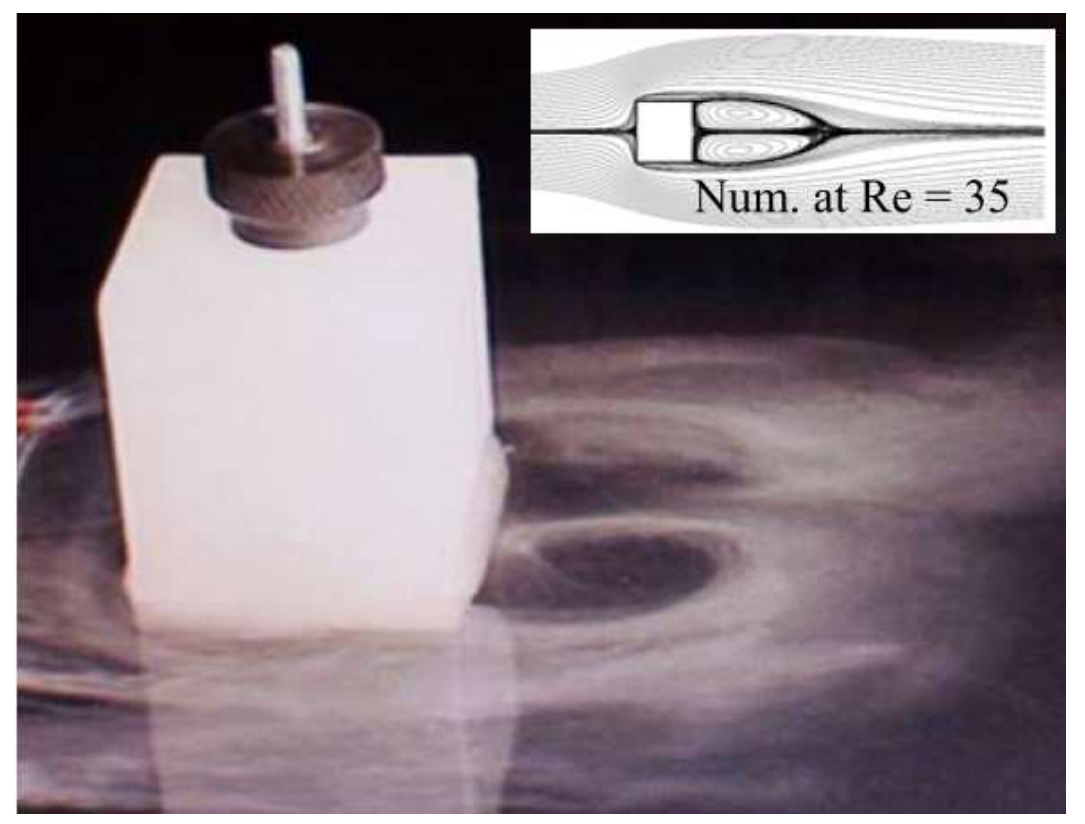

Figure 9: Hydrogen bubble visualization of flow past a square cube [127].

Addressing the problem of external flow past a square shaped bluff body, some recent research compared two different CFD techniques, namely Large Eddy Simulation (LES) and Reynolds Averaged Navier Stokes (RANS), to compute the flow around a square shaped bluff body [128]. Since LES is a time-dependent three-dimensional technique, it allowed for the visualization of the physics of the wake behind the bluff body, as shown in Figure 10(a), which is not possible to predict with the RANS method.

In another study [129], LES is used to compute unsteady flow past a square-shaped bluff body and to observe vortex shedding effects, as illustrated in Figure 11(b). The results are compared against time-averaged RANS-based simulations. It is observed that the velocity amplitude diminishes steadily with the time simulations using the 


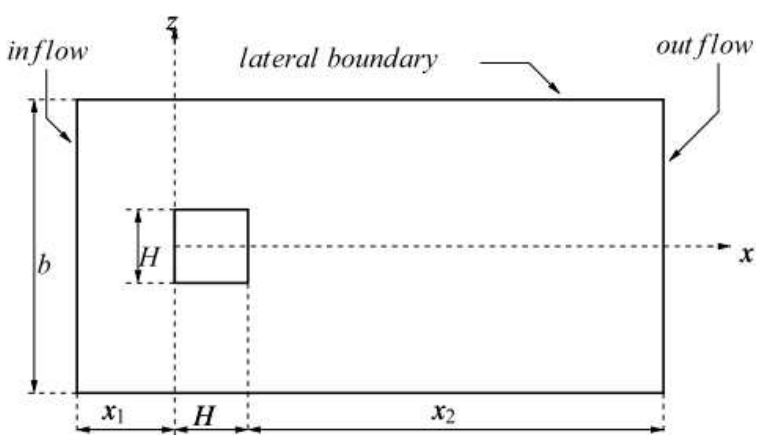

(a)

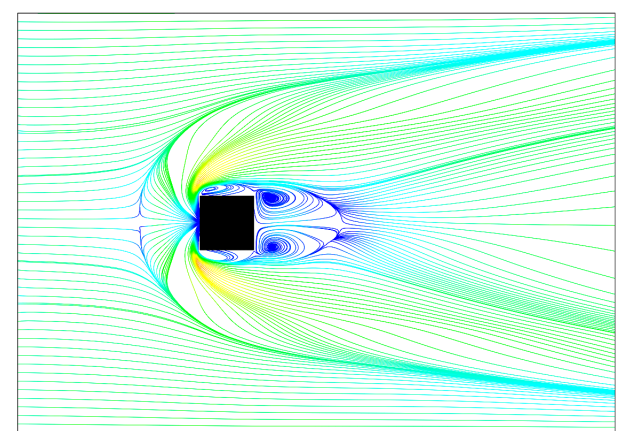

(b)

Figure 10: Large Eddy Simulation for computing flow around a square body [128]. (a) The CFD domain layout. (b) LES based flow streamlines.

$k-\epsilon$ model. Therefore, flow instabilities and vortex shedding tend to disappear in a short time and the flow becomes steady. However, LES continues to capture the vortex shedding and flow instabilities.

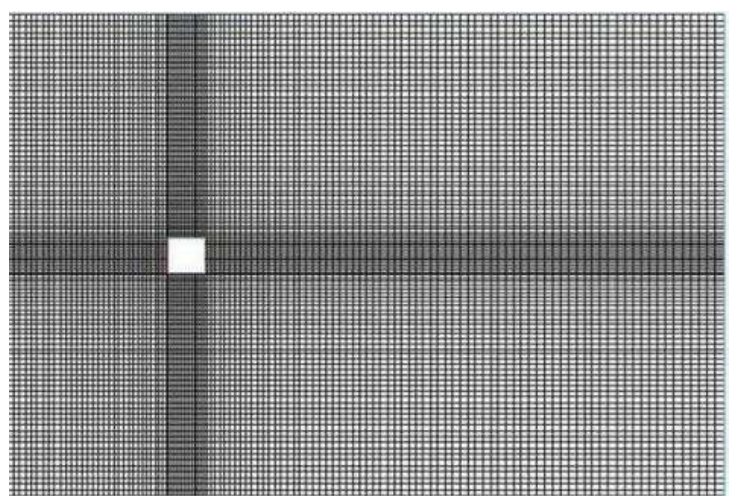

(a)

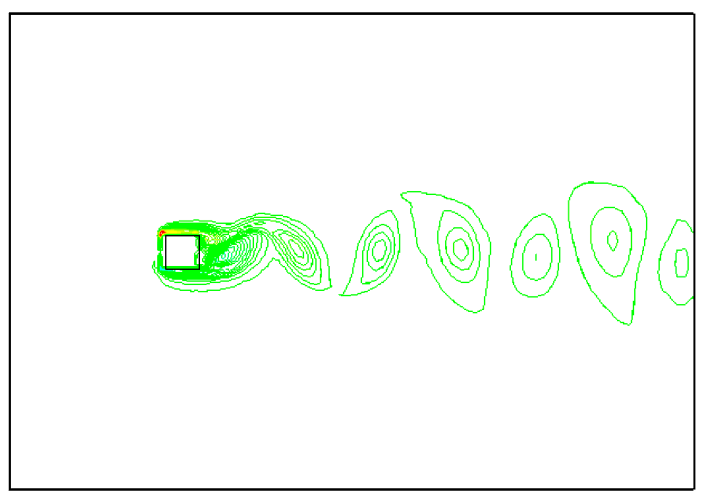

(b)

Figure 11: Large Eddy Simulation based flow around a square body [129]. (a) Non-uniform grid. (b) Capturing vortex shedding.

In the light of literature findings, CFD analysis is selected for the study of incompressible flows about three-dimensional bluff bodies to develop an understanding of wind flows past buildings. Wind flows, particularly subsonic flow regimes typical of wind speeds close to the earth's surface, are used to develop a wind gust database around three-dimensional urban structures. Furthermore, due to its ability to capture 
vortex shedding and flow instabilities, LES is chosen as the preferred technique for generating wind velocity data for the flight simulator.

The actual wind data for the flight simulator is developed in a research conducted by Sutherland [130] at Carleton University. Using a CFD software called OpenFOAM [131], the LES technique is employed to generate wind flows in a specified domain. As shown in Figures 12 and 13, the domain has a length of 117 W, a width of 58 $\mathrm{W}$, and a height of $30 \mathrm{~W}$, where $\mathrm{W}$ is a unit corresponding to the building width dimension. A single building of length to width ratio of $\mathrm{L} / \mathrm{W}=1$, and height to width ratio of $\mathrm{H} / \mathrm{W}=6$ is placed in the domain. The building centroid coordinates are $\mathrm{x}=0$, $\mathrm{y}=0, \mathrm{z}=0 \mathrm{~m}$, located at the bottom center of the building, and the incidence angle is set as $\theta_{W}=0^{\circ}$ with respect to the incoming free stream wind.

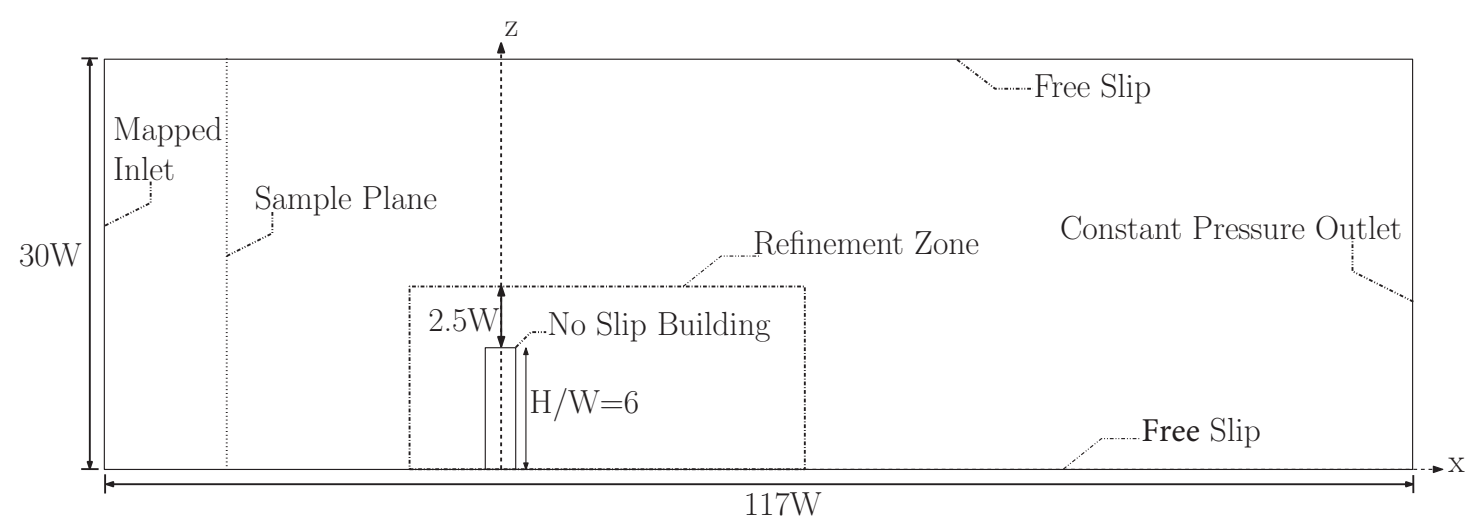

Figure 12: The CFD domain side view for the large eddy simulations [130].

A block-structured Cartesian hexahedral mesh with a total of $2.43 \times 10^{6}$ cells is used for this domain. This Cartesian mesh is refined around the building and its wake, in order to properly capture the details of turbulent flow fields. The boundary conditions for the domain walls, floor and roof are set to free slip, while the building walls are set to the no slip boundary condition. A mapped inlet boundary condition is used which samples and scales the resolved velocity components over a plane to ensure a constant bulk flow rate. The free stream velocity at the inlet is set to $4 \mathrm{~m} / \mathrm{s}$ 


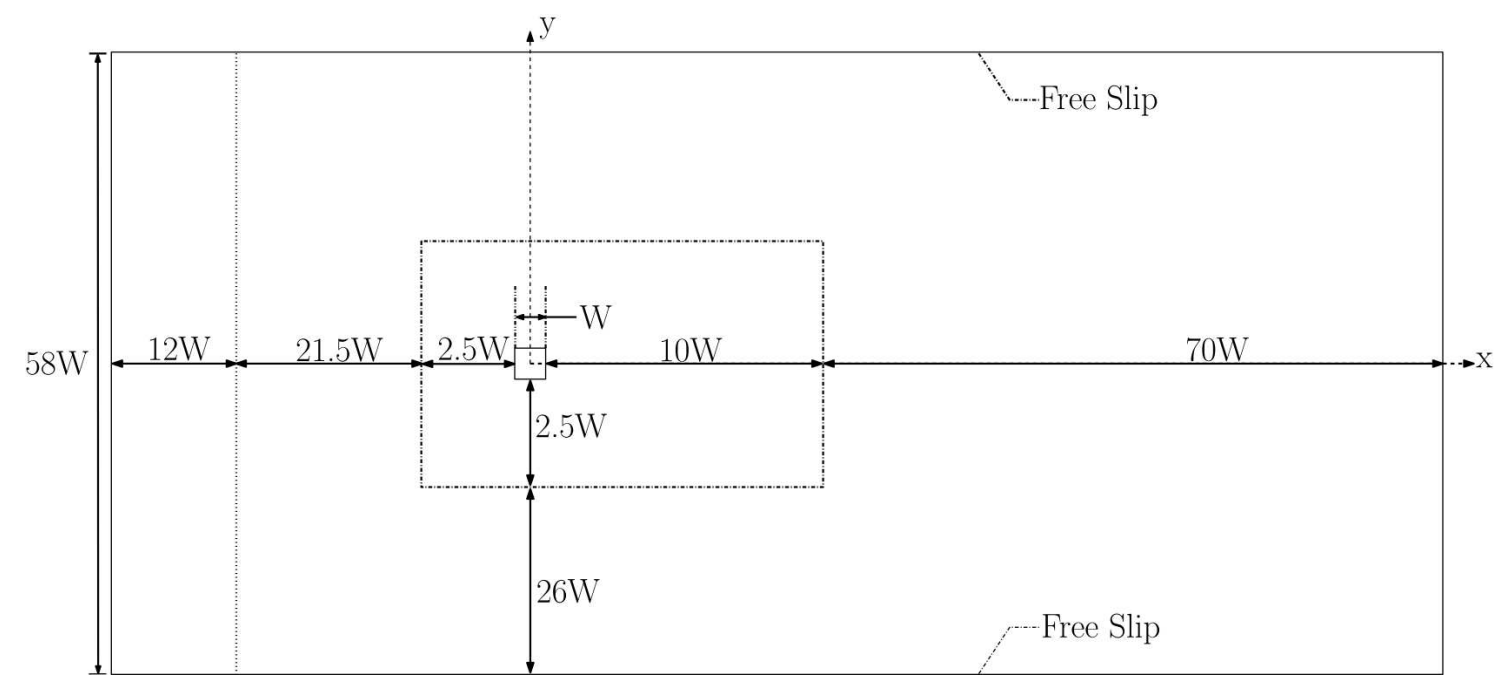

Figure 13: The CFD domain top view for the large eddy simulations [130].

and the outlet boundary condition is set to a zero static pressure. This corresponds to a Reynolds number $R_{e}=7.3 \times 10^{6}$, which is representative of an actual urban environment. A finite volume approach is used for the LES simulation by selecting a PISO [132] solver. The transient simulation is run for a total time of 30 seconds with a time step of 0.05 seconds.

CFD simulations are time consuming. The duration of a transient simulation of a 30 second flight depends upon the total number of nodes which need to be processed for each time step. A larger domain size combined with a fine resolution grid mesh results in node numbers within the order of millions, and therefore a simulation time of an order of days or weeks. To reduce the required computational time, the geometries of the domain need to be reasonably minimized, as well as grid refinements included only in areas of interest. In the present case, a scaling factor of $\frac{1}{8}$ is used for the geometries, while the freestream inlet velocity is increased by a factor of 8 , in order to achieve a Reynold's number $R_{e}=7.3 \times 10^{6}$. When using this CFD data in the simulator, all of the geometries are scaled up eight times and the wind velocities are correspondingly scaled down by the same ratio. This scaling results in a $4 \mathrm{~m} / \mathrm{s}$ 
freestream wind (ranking 3 on Beaufort scale, labeled as gentle breeze) and realistic building dimensions of $\mathrm{L}=\mathrm{W}=20 \mathrm{~m}$ and $\mathrm{H}=120 \mathrm{~m}$.

Post processing the transient analysis results for the LES simulation shows that the LES is able to capture most of the turbulent flow features, particularly unsteady vortex shedding. Vector arrows are used to visualize the separation and re-attachment zones in the front, top, lateral sides and in the wake of the building. Figure 14 shows a time capture of the wind velocity vectors in the plane of symmetry of the building, where a recirculation region on the rooftop and several vortices are visible in the wake. Different wind flow characteristics are identified and labeled as point-A to point-F in specific locations around the building. Each of these points will be discussed in detail and the wind flow features at these locations and the resulting turbulence for the quadrotor will be presented in the next section.

The post-processed results are saved such that a comma separated values (.csv) file is generated for each time step containing pressure, wind velocity values (Wn, We, Wd) as per Equation27, and position coordinates (X, Y, Z) for each node in the domain. The csv files are imported into a MySQL database (named windDB) as tables. The tables are named such that they contain the building's dimensions $L \times W \times H$, its orientation with respect to the incident wind, and the simulation time step value. Once in the database the wind velocity components at any coordinate in the domain can be obtained for any time step value using an appropriate SQL query. A Java Database Connectivity (JDBC) driver is used to allow Matlab to connect with the MySQL database server, either locally or remotely. Via this connectivity the flight simulator implemented in Simulink Matlab is able to query relevant wind velocity values from the database and apply them to TARA during flight.

The transient wakefield wind data acquired from the LES simulation is of 30 seconds duration. However, the flight simulations planned for this work are several times longer than the simulation time of the LES based wind data. Therefore, a 24 
seconds portion of the wind data is sectioned off from the total 30 second LES wind data and looped to produce a continuous wind effect. The loop interval is determined based on the transient flow features present in the velocity contours, with the start and end times selected to avoid large discontinuities as the data is looped back.

\subsection{Single Building Test Points}

Wind gust conditions in urban areas change based on the configuration or arrangement of urban structures in the path of the background wind. As the over-riding wind is redirected by these urban structures, the result is regions with increased or decreased wind speeds, and in some cases reversal of wind flow. Several flight scenarios can emerge from permutations of different-sized structures in various arrangements. This research starts with a very basic scenario of a single building and the wind gust conditions in the wake of this structure. Potential wind disturbance areas such as recirculation zones above the building rooftops, a wake eddy located downwind some distance from the building, and the accelerated flow due to side streams near the building edges are hazardous for a quadrotor flight.

A single building case can fit several realistic practical application scenarios, such as inspecting and classifying tall structures [133], roofing inspections [134], washing of windows and maintenance of a building using a hovering UAV to fly and make

physical contact with the structure [135]. Similarly, a fire rescue example [136] may require a UAV to look inside a window at a certain height, or a UAV can be used by the movie or media industry [137] for shooting aerial footage close to tall structures. Another example is collecting emission samples from industrial smoke stacks [138], which would require UAV to fly in close proximity of chimney, which is analogous to flying next to the rooftop of a building.

An evaluation of the performance of an autonomous position controller in the 
presence of such wind gust conditions is necessary. Therefore, several different location points are selected in the wake of a single building where the nature of wind gust disturbance is of particular interest. The results of LES-based transient wind flow around a single building help in the identification of hazardous areas and in choosing specific flight test locations, identified as point-A to point-F in Figure 14. Contour plots of wind velocity are superimposed on wind velocity vectors to provide details about the flow features around the building. These selected locations cover most of the real life practical application scenario examples presented earlier.

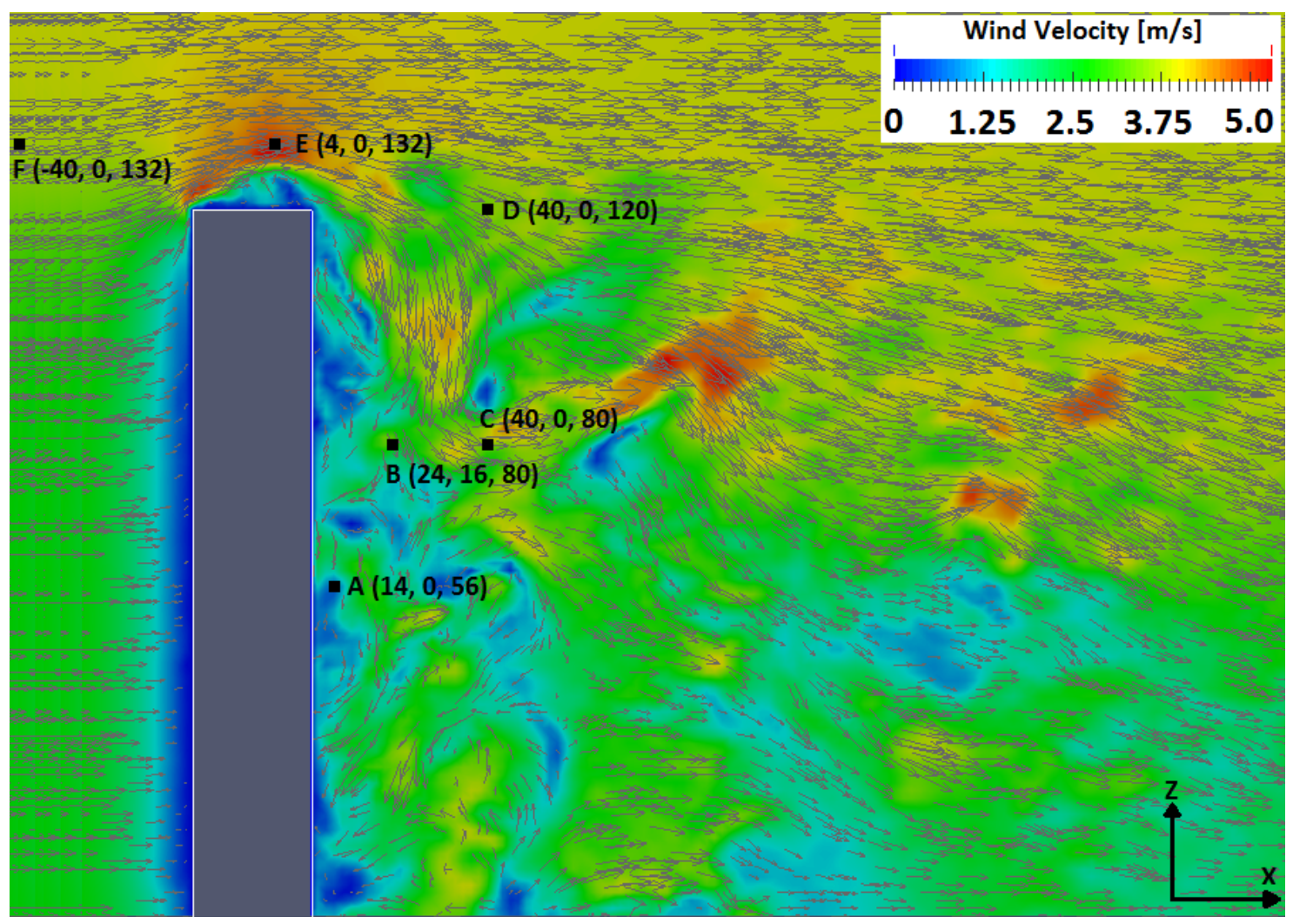

Figure 14: Test points A to F around the symmetry plane of a single building.

In order to evaluate and compare the performance of different control techniques, a consistent setup needs to be devised that will subject each controller to a series of test cases and to observe different aspects of the control performance. The results 
of these test cases will be evaluated using established criteria which reflect desired performance expectations. The following subsections discuss each of these flight test cases in detail.

\subsubsection{Dead Air Zone (Point-A)}

Point-A is in the leeward side of the building at $\mathrm{X}=14, \mathrm{Y}=0$, and $\mathrm{Z}=56$ meters (see Figure 15) and based on the measured wind velocities, is situated in the low-speed part of the wake. As the accelerated side streams produce horseshoe vortices in the wake, they leave behind a dead air zone with chaotic airflow. Therefore, while evaluating the position control in the presence of disturbances ranging from mild to high, in test cases 2 to 5 , it would be logical to observe position control performance in a chaotic flow region with a near zero average velocity. As shown in Figure 16, the $\mathrm{X}$-axis wind is averaged at $-0.3 \mathrm{~m} / \mathrm{s}$, fluctuating between -2.0 and $2.3 \mathrm{~m} / \mathrm{s}$. The Y-axis wind velocity is also averaged around zero with fluctuations of -3.2 and 3.0 $\mathrm{m} / \mathrm{s}$. The Z-axis wind velocity is averaged at $-0.5 \mathrm{~m} / \mathrm{s}$ and fluctuates between -2.7 to $1.8 \mathrm{~m} / \mathrm{s}$.

\subsubsection{Passing Vortex (Point-B)}

The region around point- $\mathrm{B}$ at coordinates $\mathrm{X}=24, \mathrm{Y}=16$, and $\mathrm{Z}=80$ meters is important because this is where the first vortices form due to the accelerated flow by the side sub-streams. A top-down view in Figure 17(a), shows two horseshoe-like vortices forming along the gradient line $G 2$ due to the symmetry of the flow. Because of the transient nature of the flow, these vortices move farther downstream in a phenomenon called vortex shedding. As the vortex shedding occurs the moving vortex loses energy and diminishes in its strength, therefore the first vortex is considered to be of greatest interest, being close to the building with significant turbulent effect. Also 


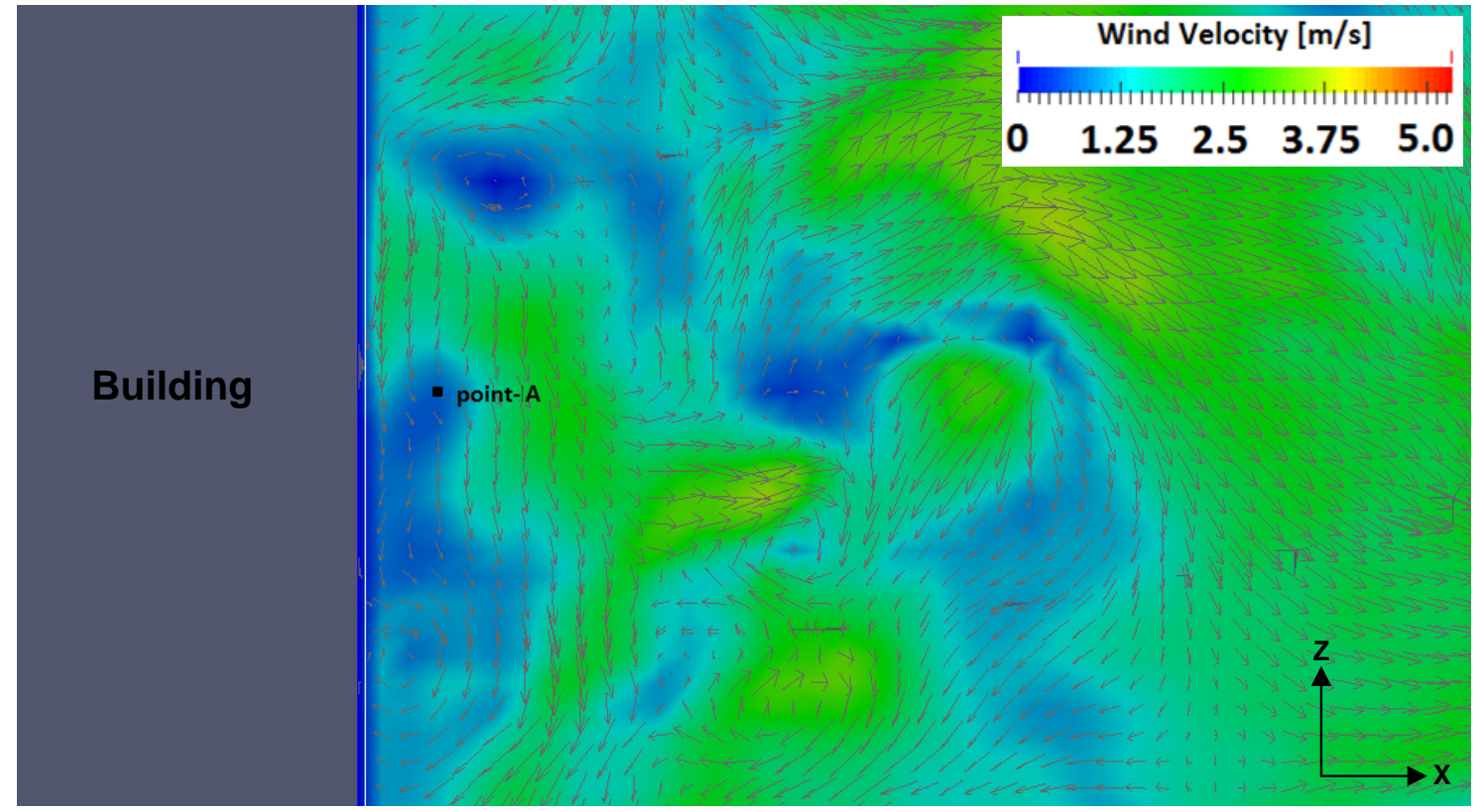

(a)

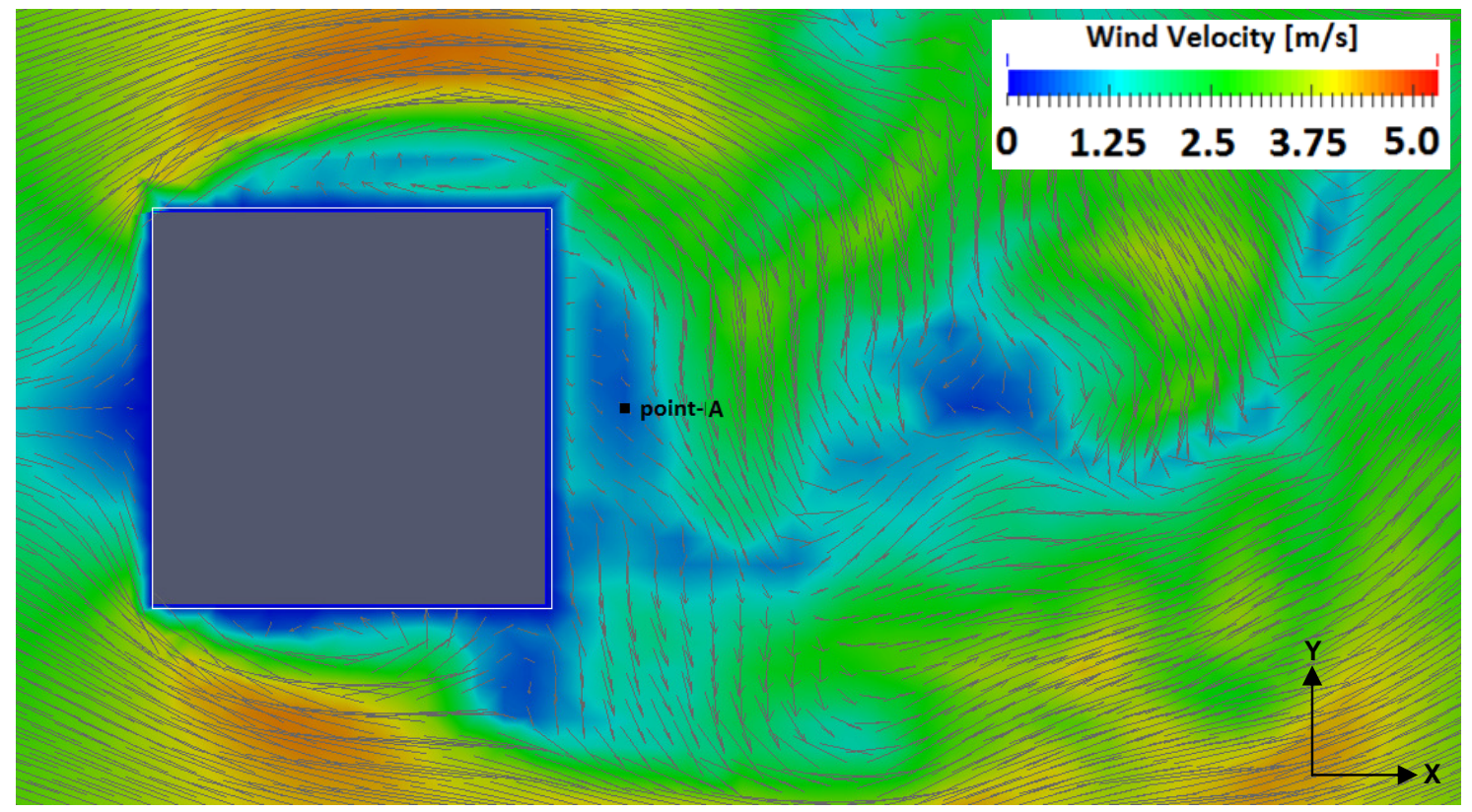

(b)

Figure 15: Wind flow at point-A. (a) Side view showing low wind velocities. (b) Top view showing point-A close to the building.

worth noting is that the flow at point-B is of three-dimensional nature, as shown by a side-view in Figure 17(b). The X-axis wind, as depicted in Figure 18(a), is averaged 


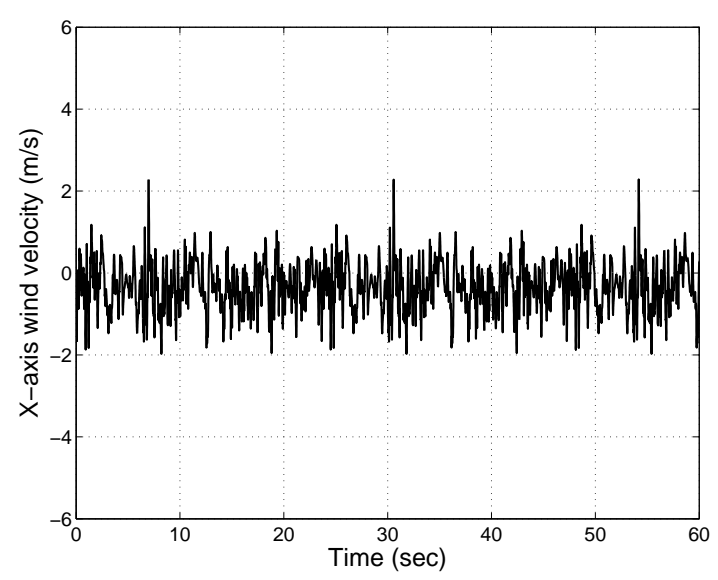

(a)

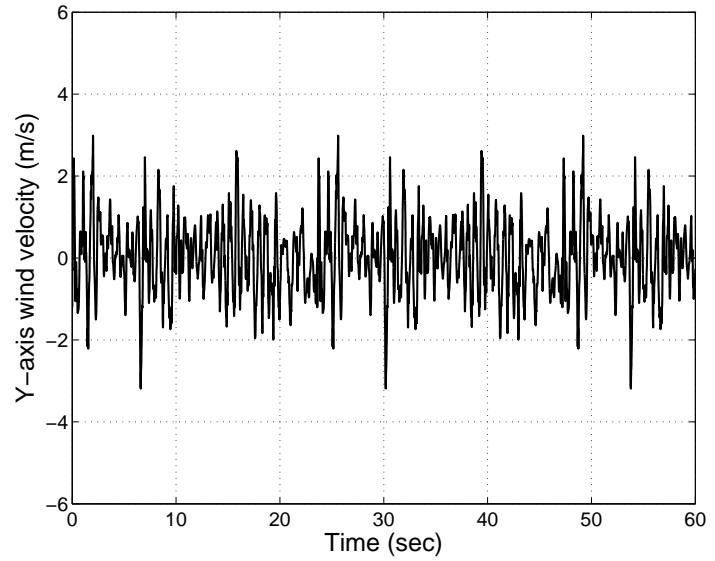

(b)

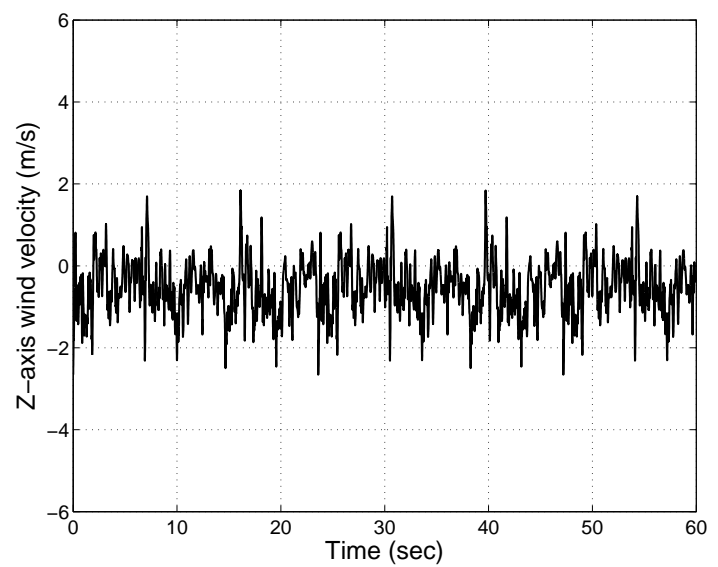

(c)

Figure 16: Wind velocities at point-A. (a) X-axis. (b) Y-axis. (c) Z-axis.

at $2.5 \mathrm{~m} / \mathrm{s}$ and fluctuates between values of 5.7 to $-2.2 \mathrm{~m} / \mathrm{s}$ as the vortex passes this point. The Y-axis wind velocity fluctuates between values of $\pm 2.9 \mathrm{~m} / \mathrm{s}$ as shown in Figure 18(b) where again, as the leading and trailing edges of the vortex pass its point, large changes in the $\mathrm{Y}$-axis velocity are achieved. The Z-axis wind as shown in Figure $18(\mathrm{c})$ is averaged at $0.2 \mathrm{~m} / \mathrm{s}$ with fluctuations of $\pm 2.7 \mathrm{~m} / \mathrm{s}$ similar to point-A. 


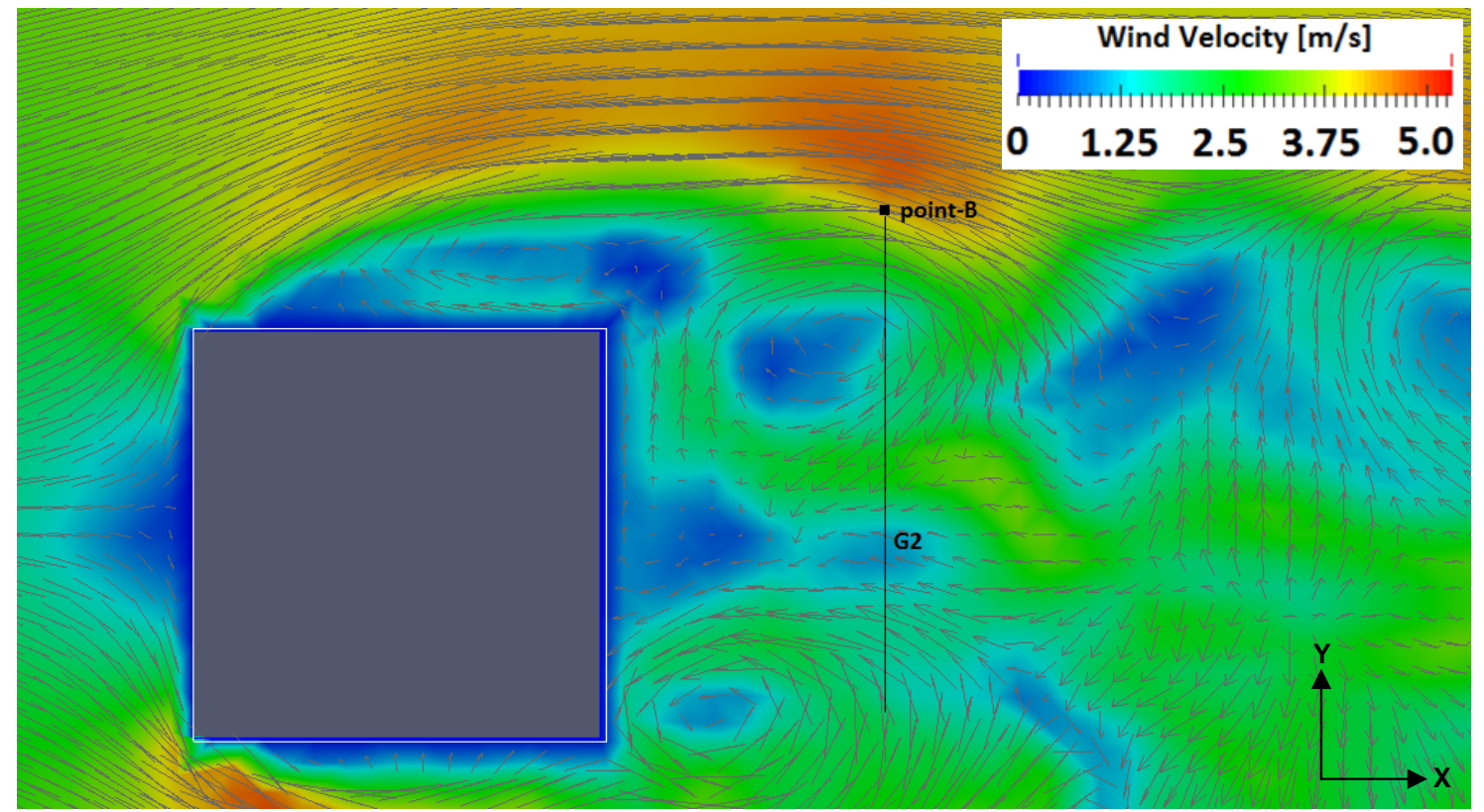

(a)

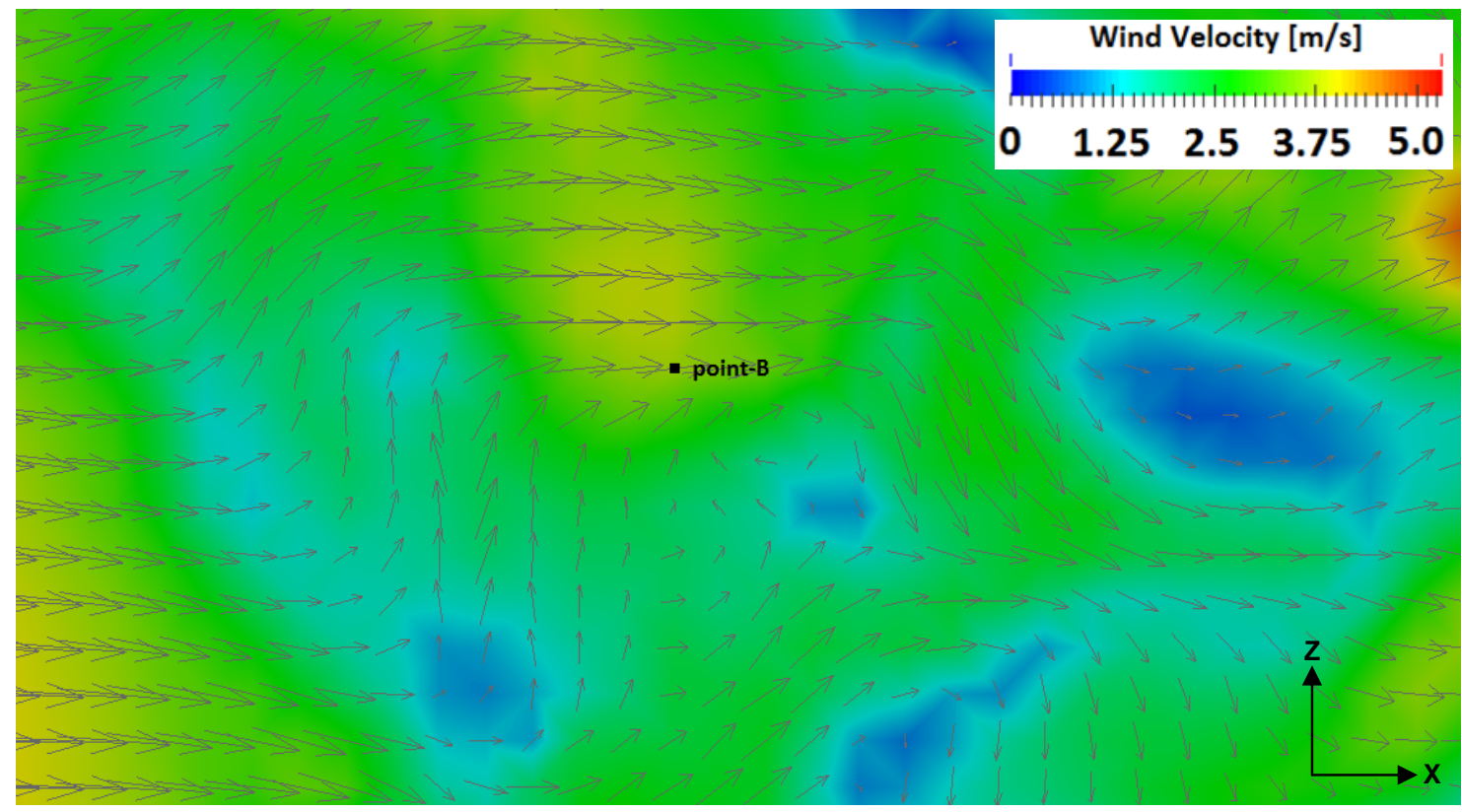

(b)

Figure 17: Wind flow at point-B. (a) Top view showing the horseshoe vortices. (b) Side view showing the nature of the flow. 


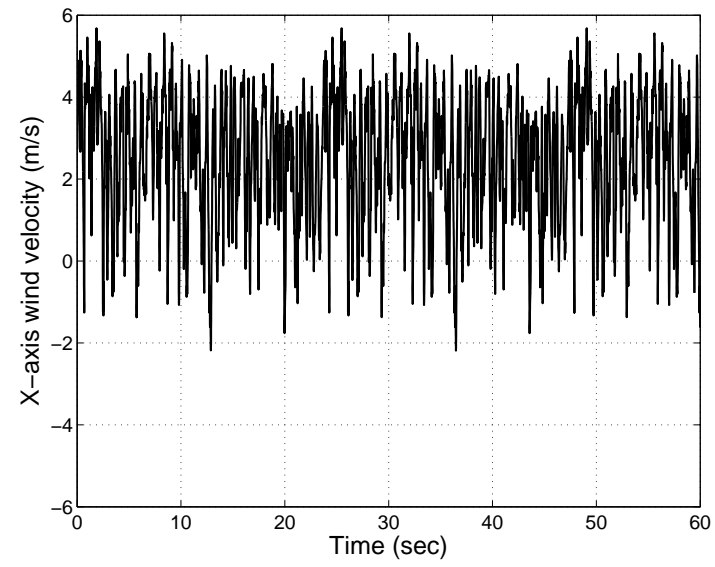

(a)

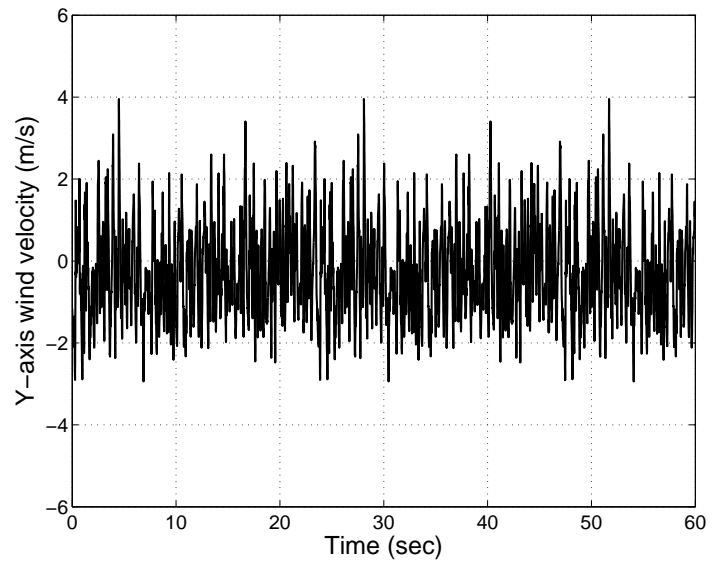

(b)

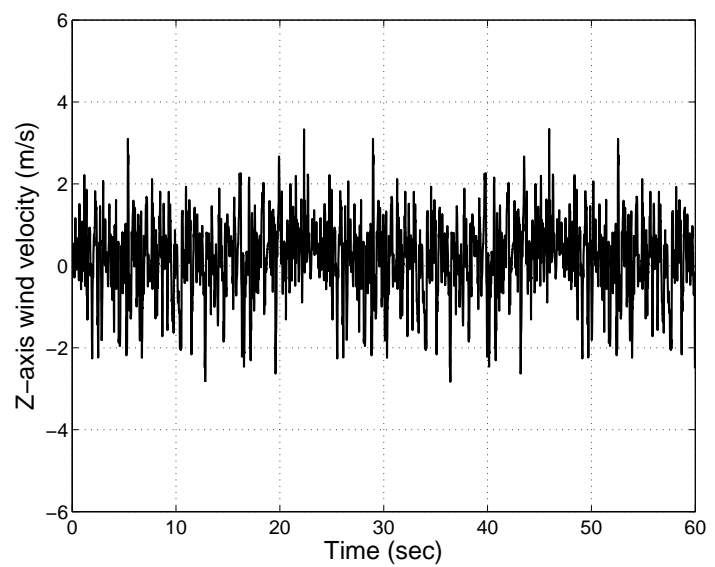

(c)

Figure 18: Wind velocities at point-B. (a) X-axis. (b) Y-axis. (c) Z-axis.

\subsubsection{Wake Eddy (Point-C)}

Point- $\mathrm{C}$ at the coordinates $\mathrm{X}=40, \mathrm{Y}=0, \mathrm{Z}=80$ meters, is located where three substreams meet and produce a swirling of the flow known as a wake eddy. As mentioned in previous subsection, the two side sub-streams forming vortices close to point-B result in vortex shedding downstream. Adding to this, as the sub-stream accelerating over the roof top descends some distance from the building, it combines with the shedding vortices and forms a wake eddy. Figures 19(a) and 19(b), show the topdown and side views of the wind flows around point-C. The $\mathrm{X}$-axis wind velocity 
as shown in Figure 20(a) is averaged at $0 \mathrm{~m} / \mathrm{s}$ and fluctuates between 3.6 and -2.3 $\mathrm{m} / \mathrm{s}$. The Y-axis wind velocity shown in Figure $20(\mathrm{~b})$ is also averaged at $0 \mathrm{~m} / \mathrm{s}$ and fluctuates between -4.6 and $4.0 \mathrm{~m} / \mathrm{s}$. The Z-axis wind velocity, shown in Figure 20(c), is averaged at $-0.9 \mathrm{~m} / \mathrm{s}$ and fluctuates between 2.6 and $-4.4 \mathrm{~m} / \mathrm{s}$ values. A position hold at point-C will be used to evaluate the handling by a control technique of the three-dimensional disturbances produced in the wake eddy.

\subsubsection{Downwash (Point-D)}

Located above the wake eddy at $\mathrm{X}=40, \mathrm{Y}=0, \mathrm{Z}=120$ meters point- $\mathrm{D}$, shown in Figure 21(a), is influenced by the descending high-speed sub-stream coming over the rooftop. Wind shear effects are evident along the gradient line G3 depicted in Figure 21(b). The wind disturbance at this location is mostly two-dimensional. The wind velocity on the $\mathrm{X}$-axis averages at $3.1 \mathrm{~m} / \mathrm{s}$ and fluctuates between 4.4 to $0.4 \mathrm{~m} / \mathrm{s}$ values as shown in Figure 22(a). The Y-axis wind velocity is averaged around zero and fluctuates between -2.9 and $2.2 \mathrm{~m} / \mathrm{s}$ as shown in Figure 22(b). The Z-axis wind is the rooftop sub-stream descending in the wake and as shown in Figure 22(c). An average wind velocity of $-1.0 \mathrm{~m} / \mathrm{s}$ is present on the Z-axis which fluctuates between 1.2 and $-2.2 \mathrm{~m} / \mathrm{s}$ values. In this case the wind velocity bias along the $\mathrm{X}$-axis would push a quadrotor away from the building, while the downward biased flow will affect the altitude control efforts.

\subsubsection{Accelerated Freestream (Point-E)}

As the freestream incoming wind hits the flat face of building wall, the flow splits into four sub-streams, one sub-stream going over the rooftop, two sub-streams going around the sides, and the last one moving downward towards the ground. The pointE location at $\mathrm{X}=4, \mathrm{Y}=0$, and $\mathrm{Z}=132 \mathrm{~m}$, as shown in Figure 23(a), is deliberately 


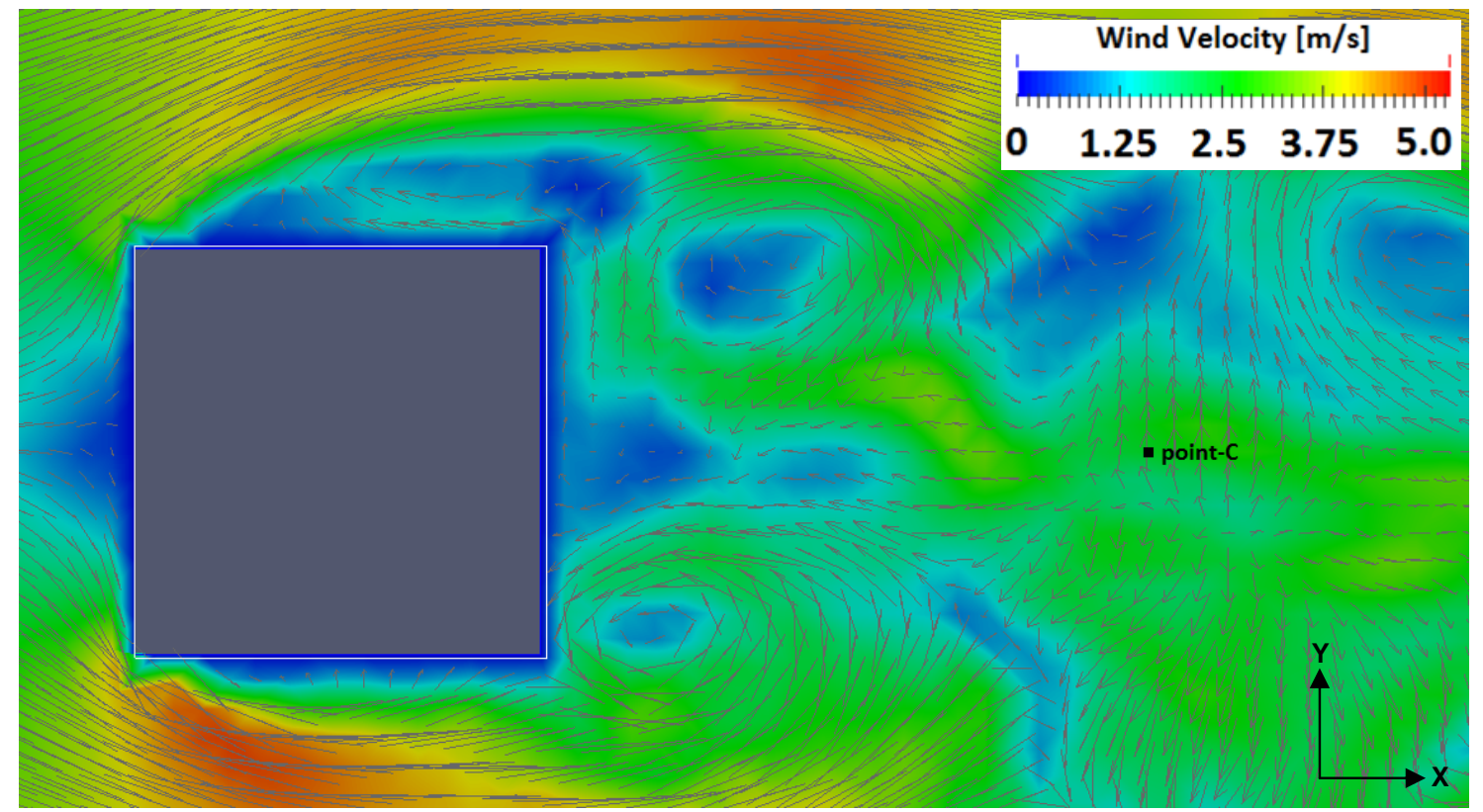

(a)

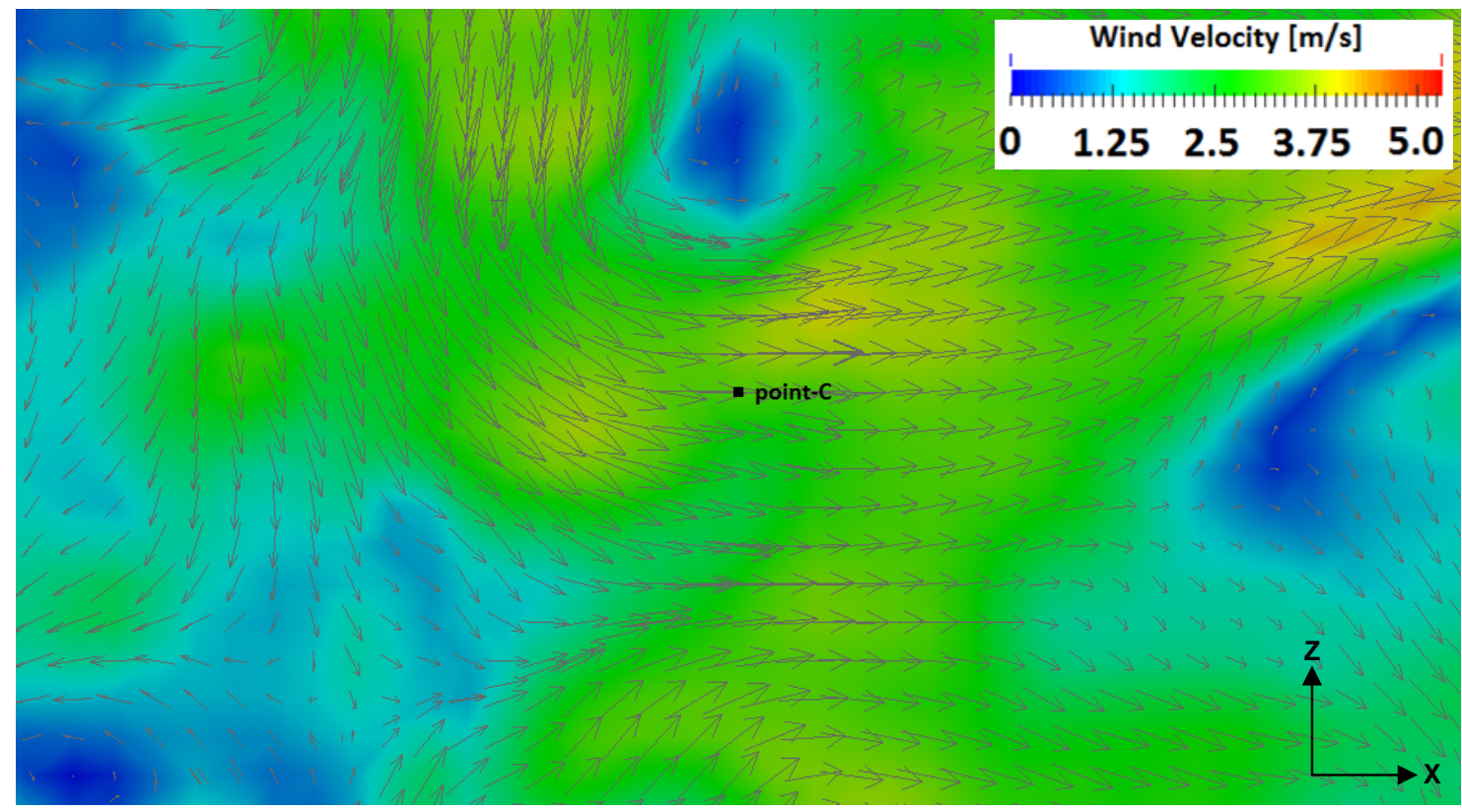

(b)

Figure 19: Wind flow at point-C. (a) Top view showing the wake eddy. (b) Side view showing converging of top and side sub-streams.

positioned close to the recirculation zone formed by the sub-stream going over the rooftop. The wind velocity along the X-axis, shown in Figure 24(a), is the highest 


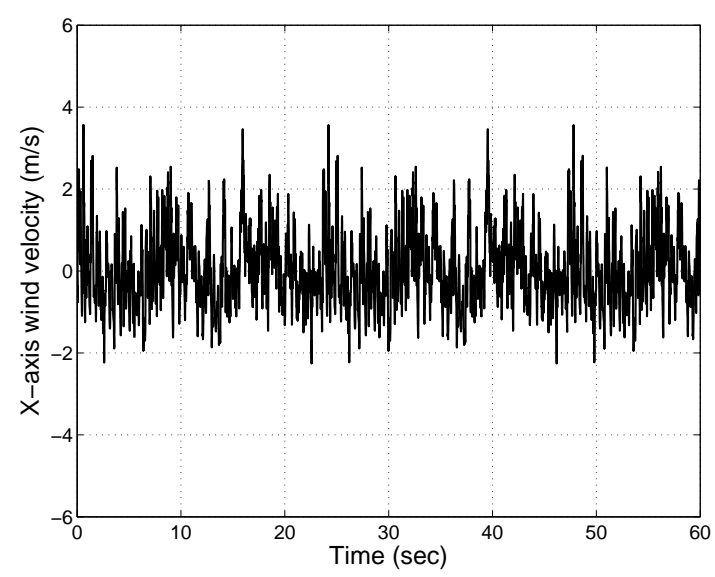

(a)

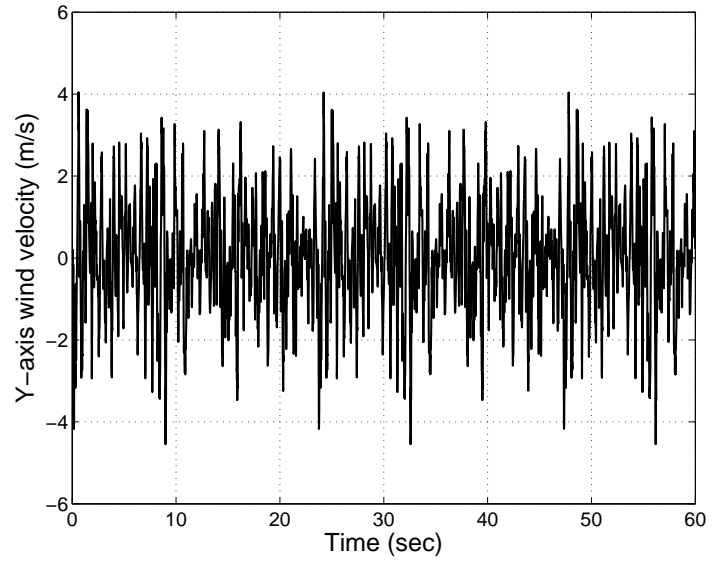

(b)

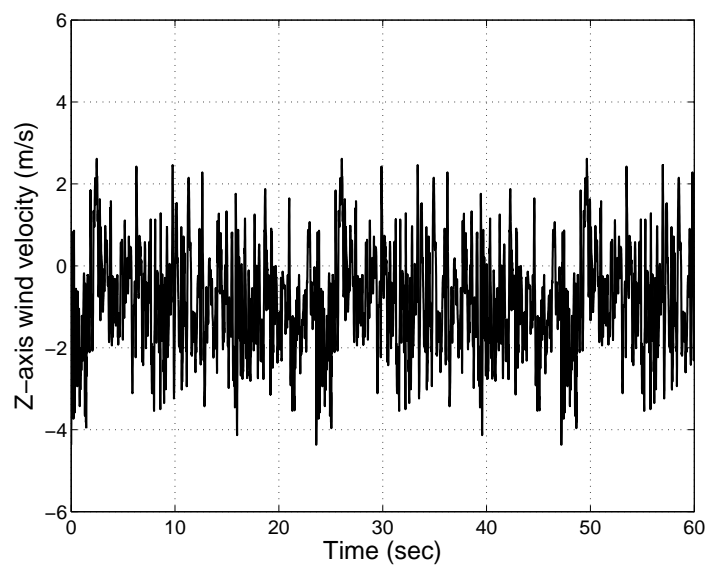

(c)

Figure 20: Wind velocities at point-C. (a) X-axis. (b) Y-axis. (c) Z-axis.

among the test cases, averaging around $4.5 \mathrm{~m} / \mathrm{s}$, fluctuating between 3.6 and 5.2 m/s. The wind disturbance on the Y-axis, as shown in Figure 24(b), is averaged around zero with small fluctuations of $\pm 0.4 \mathrm{~m} / \mathrm{s}$. The Z-axis wind velocity averages at $0.1 \mathrm{~m} / \mathrm{s}$ with ranging between -0.5 and $1.4 \mathrm{~m} / \mathrm{s}$, as shown in Figure 24(c). The recirculation zone below point-E shows complete reversal of flow along the $\mathrm{X}$-axis. To elaborate on the nature of the disturbances present in this region, Figure 23(a) shows a gradient line $G 1$ going through the recirculation zone. The position hold at point-E poses the challenge of position stabilization in the presence of strong wind conditions 


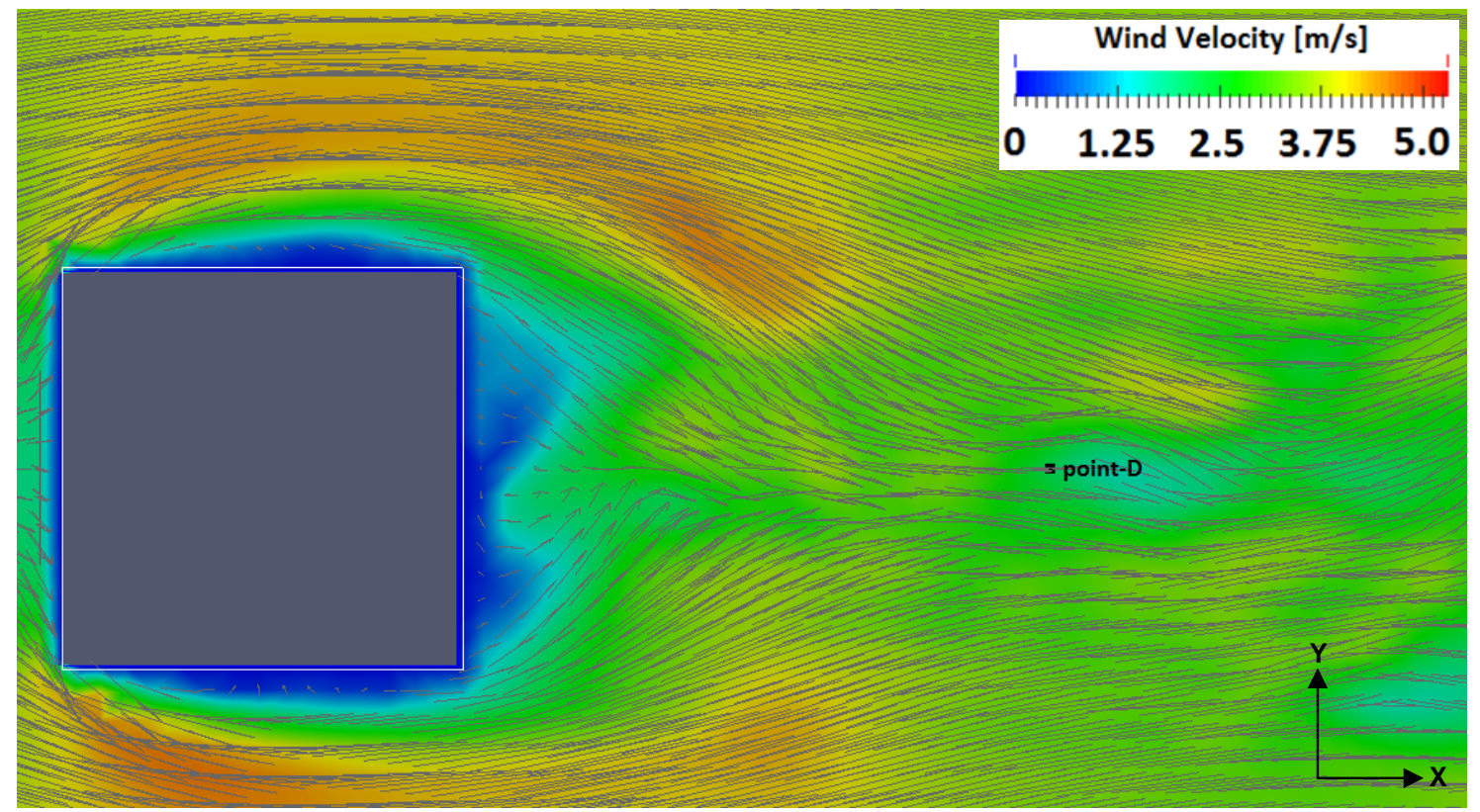

(a)

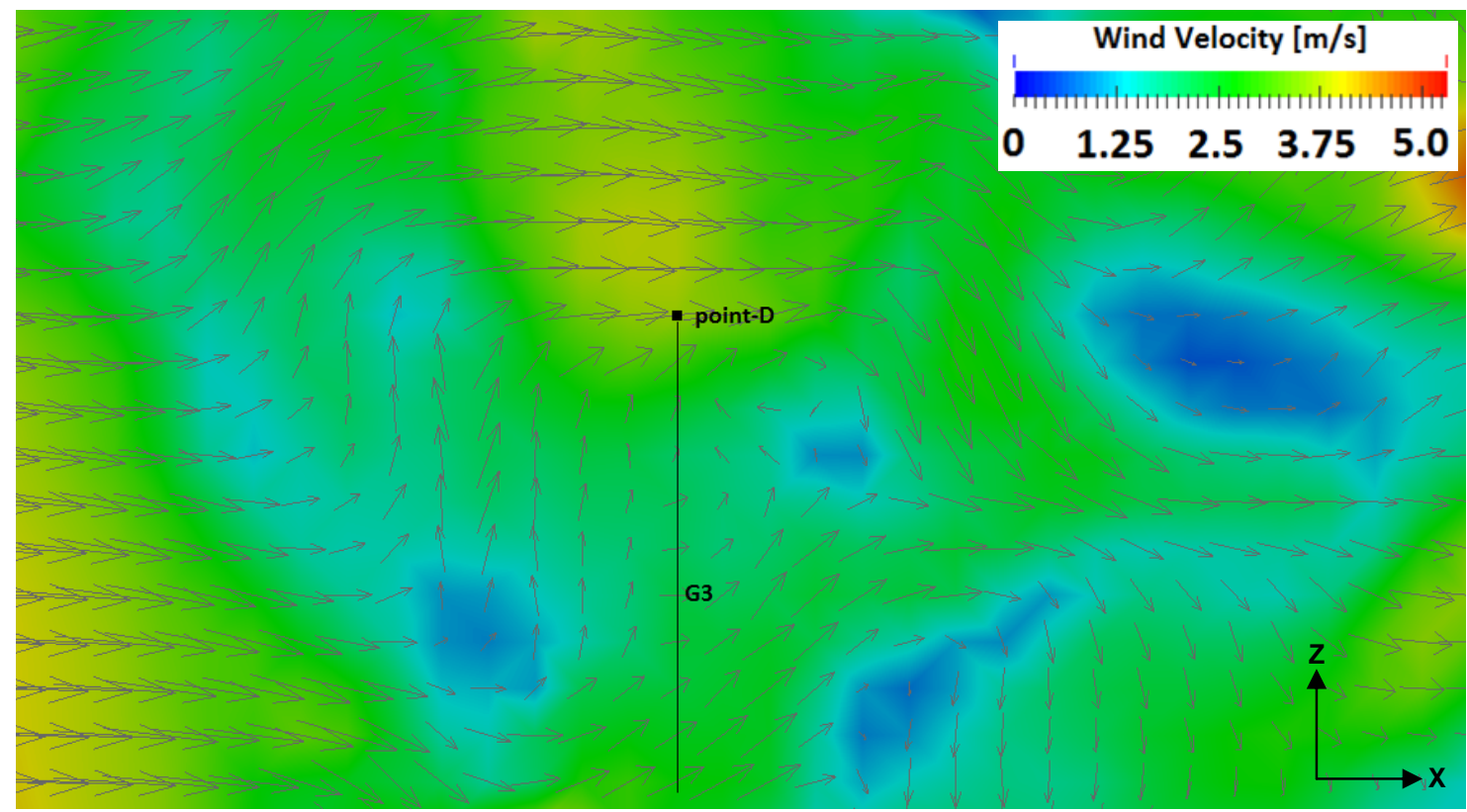

(b)

Figure 21: Wind flow at point-D. (a) Top view showing the wind velocity along $\mathrm{X}$-axis. (b) Side view showing wind shear.

but smaller turbulent fluctuations. 


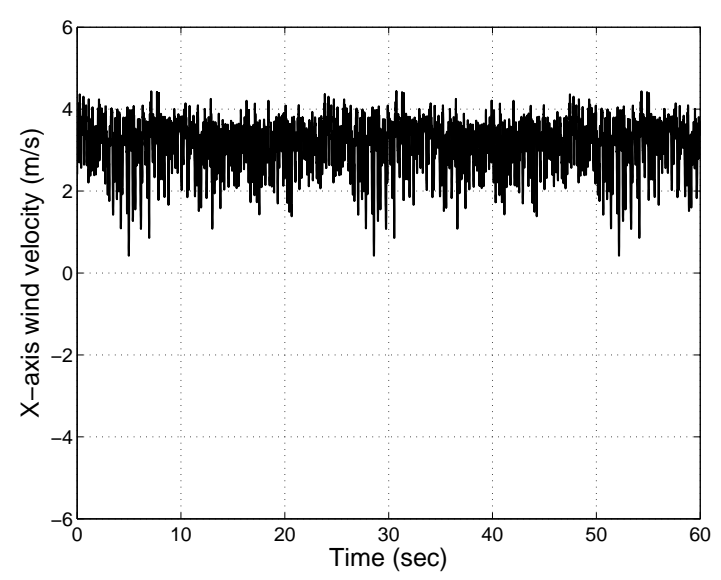

(a)

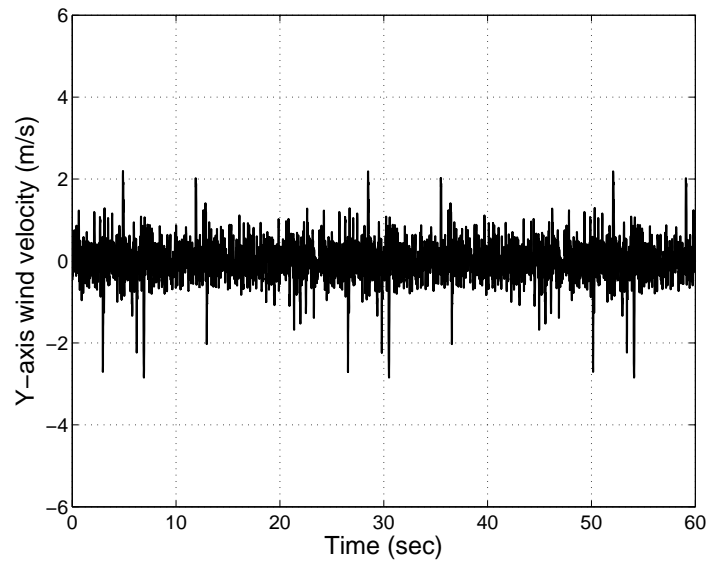

(b)

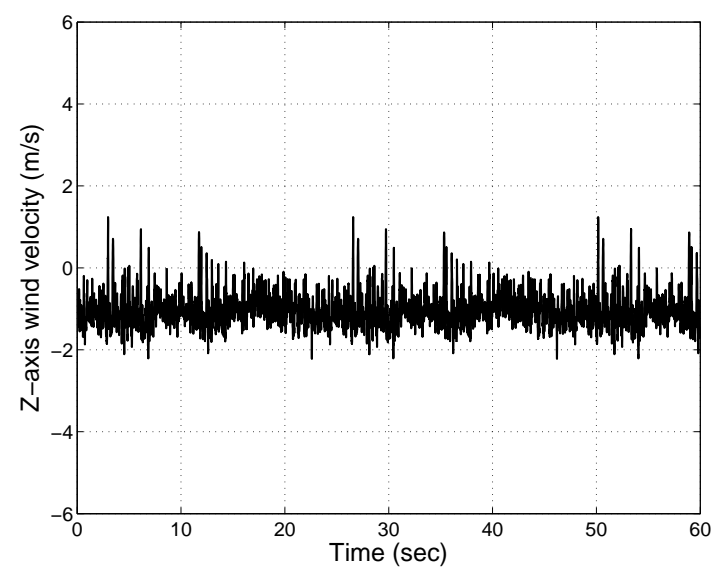

(c)

Figure 22: Wind velocities at point-D. (a) X-axis. (b) Y-axis. (c) Z-axis.

\subsubsection{Freestream Wind (Point-F)}

Finally, a flight test in the freestream wind before the building at coordinates $\mathrm{X}=-40$, $\mathrm{Y}=0$, and $\mathrm{Z}=120$ meters, will place TARA in a similar scenario to a typical outdoor flight in an open area. The wind in such cases as shown in Figure 25, is mostly unidirectional, and constant in nature with minor fluctuations. In this particular case the main wind flow is along the X-axis (Figure 25(a)) with $4 \mathrm{~m} / \mathrm{s}$ average velocity. 


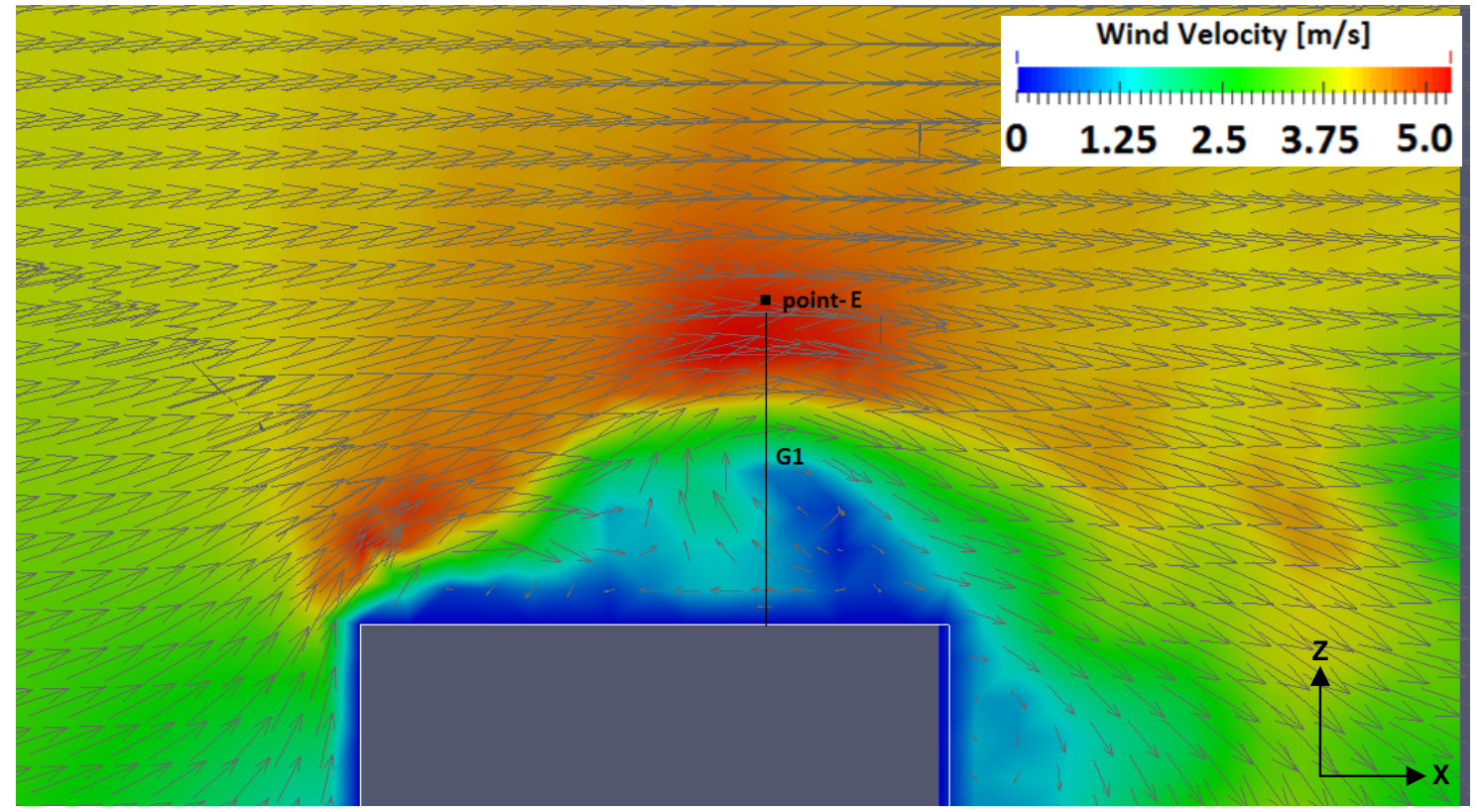

(a)

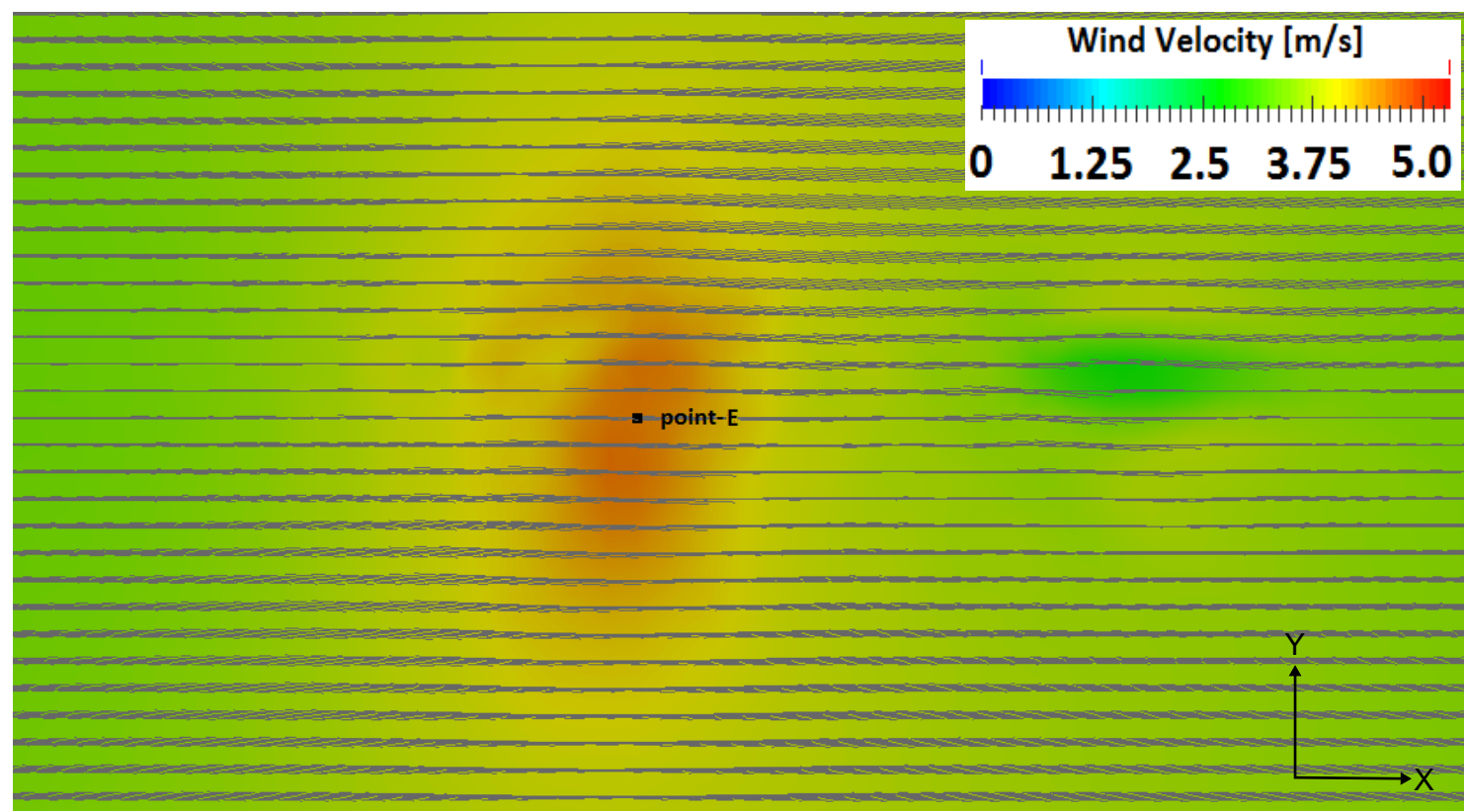

(b)

Figure 23: Wind flow at point-E. (a) Side view showing the recirculation zone. (b) Top view showing prominently $\mathrm{X}$-axis flow. 


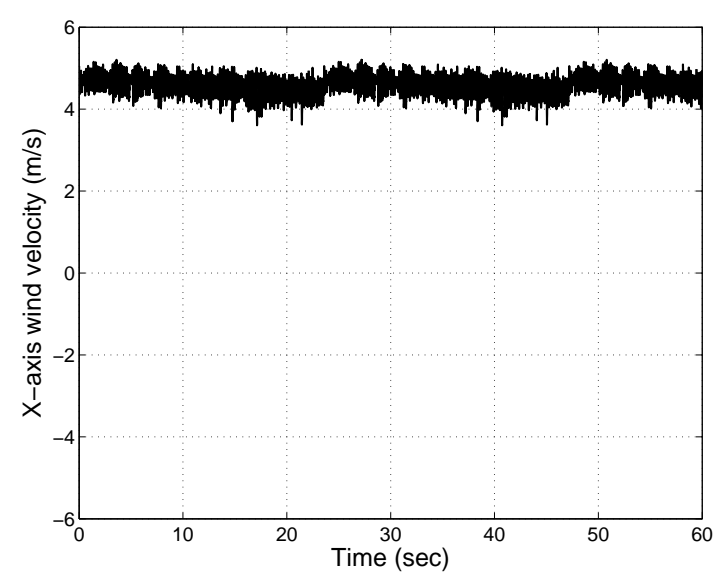

(a)

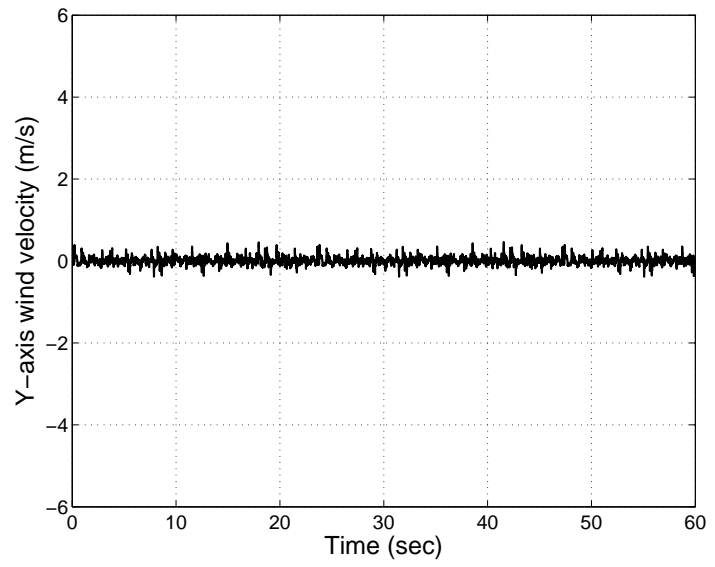

(b)

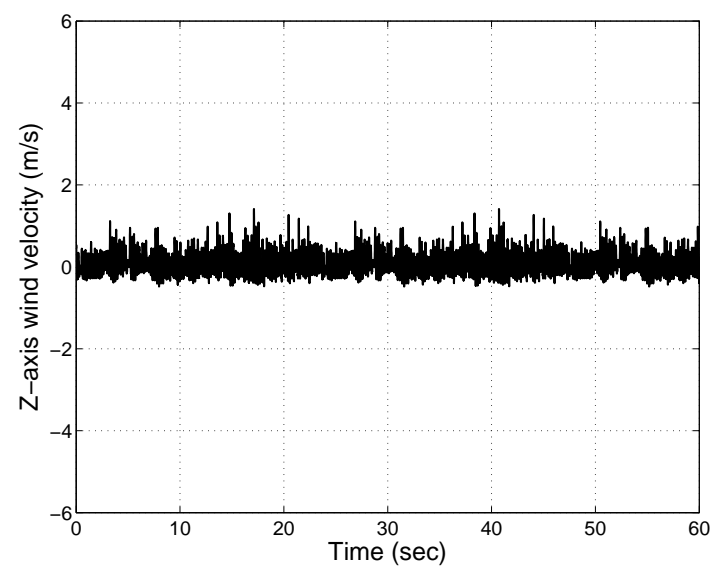

(c)

Figure 24: Wind velocities at point-E. (a) X-axis. (b) Y-axis. (c) Z-axis.

\subsection{Criteria for Evaluating and Comparing Con- trol Performances}

Based on the test cases defined in the previous section, TARA will be programmed to fly through a pre-defined way-point scheme and perform position holds on points A to F. A total time of 720 seconds will be used for the flight simulation such that first 60 seconds are allocated for TARA to takeoff from ground at $\mathrm{X}=14, \mathrm{Y}=0$, and $\mathrm{Z}=0$ to reach point-A. Position hold for point-A is evaluated for a full one minute, 


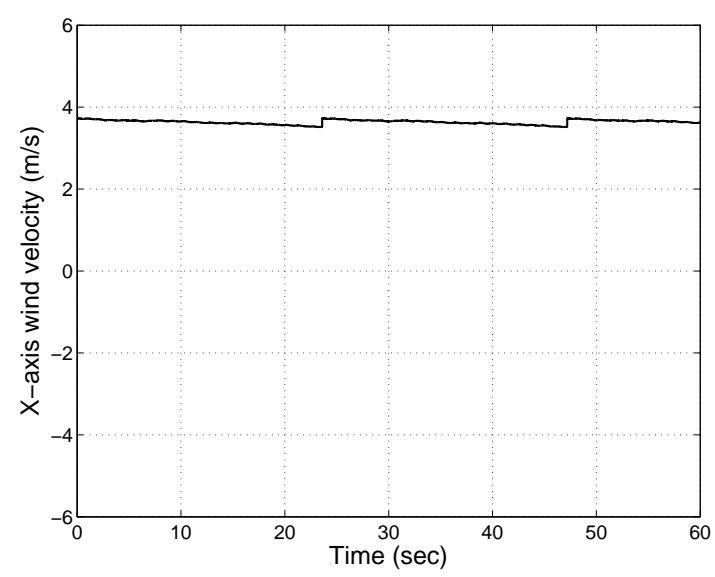

(a)

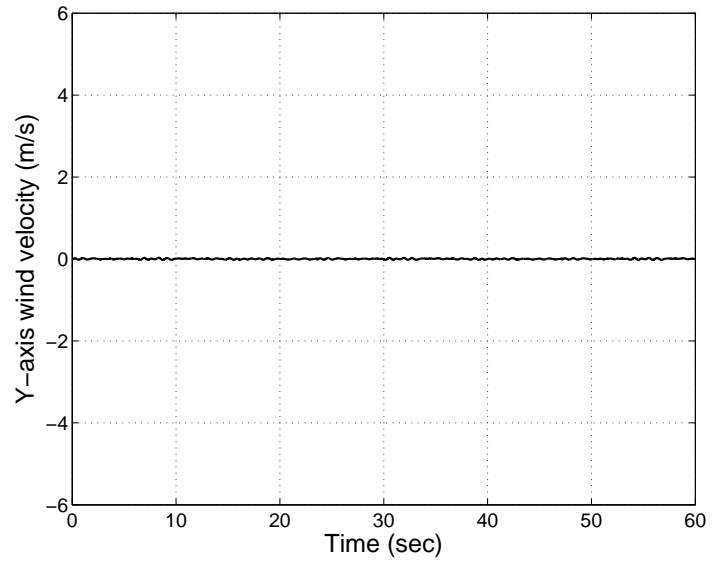

(b)

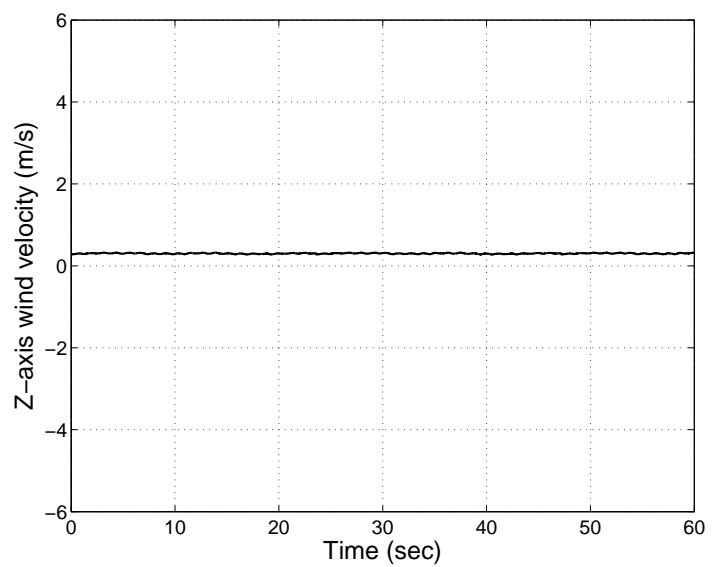

(c)

Figure 25: Wind velocities at point-F. (a) X-axis. (b) Y-axis. (c) Z-axis.

starting from the time mark of 60 seconds to 120 seconds. After the end of position hold at point-A, TARA is allowed a full one minute to move from point-A to point-B. Therefore, a position hold at point-B is evaluated between time marks of 180 seconds to 240 seconds. Similarly, point-C between 300-360 seconds, point-D between 420-480 seconds, point-E between 540-600 seconds, and point-F between 660-720 seconds, are evaluated for respective position holds. Also, step response is evaluated for each axis by programming TARA to move from one point to another. Different position control techniques will be used to carry out the same flight maneuver. This section details 
the methods and development of fixed criteria to evaluate the performance of these different control techniques.

Because of the disturbances present, two flight simulations using different control techniques will not be exactly the same. This is due to the fact that the particular position of TARA at an instant in time will result in specific wind velocity values. Therefore, unless two control techniques produce an identical flight trajectory with respect to time, the wind disturbances experienced will not be identical. In the light of this fact, the flight data resulting from a test case will be analyzed by two methods. Ultimately, the goal is to be able to objectively rate the various control techniques applied and effectively evaluate which can be considered to provide the best performance.

To begin, as a gross measure of a controller's ability to maintain position, the absolute bounds of the flight envelope will be calculated. When considering an urban flight scenario, flying in close proximity to buildings and other structures, it is important to identify if the flight envelope coincides with the location of any such obstacles. To further evaluate the maximum error bound achieved, a performance metric, shown in Table 2, is established based on literature and experimental data. A position control is considered to be "good" if the quadrotor's position is maintained within \pm 1 $\mathrm{m}$ of the desired coordinates. This represents two body lengths of TARA which has dimensions $0.55 \times 0.55 \times 0.085$ meters. An "acceptable" control performance is one resulting in an error range of $1<$ error $\leq 3 \mathrm{~m}$. A "poor" control performance error range is determined to be $3<$ error $\leq 5 \mathrm{~m}$. If the error range is determined to be $>5 \mathrm{~m}$ then the control performance is considered "bad". If the error is growing over time then an "unstable" behavior is declared.

While the bounds of the flight envelope provide a useful assessment of a particular control technique, the nature of this measurement essentially characterizes a flight controller in terms of its worst error performance. A controller which allows for 
Table 2: Position control performance metric.

\begin{tabular}{|c|c|c|c|c|c|}
\hline Absolute Error $(\mathrm{m})$ & $\leq 1$ & $1<$ error $\leq 3$ & $3<$ error $\leq 5$ & $>5$ & Not Converge \\
\hline \hline Performance Grade & Good & Acceptable & Poor & Bad & Unstable \\
\hline
\end{tabular}

a brief but relatively high initial error before successfully bringing and holding the quadrotor in the desired position would be penalized in comparison with another controller which produces a consistent mid-range error in flight position without ever approaching the desired coordinates. To effectively characterize such varying flight scenarios, an average of absolute difference (AOAD) metric will also be employed, which is sometimes also referred to as the mean absolute error. For each time step, the absolute difference between the desired and actual position will be determined. The average of these differences will then be calculated to produce a single numerical value for each flight, per Equation 33.

$$
\bar{\Phi}=\sum_{i=1}^{n} \frac{\left|\Phi_{\text {desired }}^{i}-\Phi_{\text {actual }}^{i}\right|}{n}
$$

Unlike many other commonly employed metrics, such as Integral Squared Error (ISE) or Root-Mean-Square Error (RMSE) measures, both the maximum error and AOAD metrics selected for the evaluation of flight performance provide numerical values which are intuitive to understand and readily correlate with the real time flight experience. Furthermore, considering both maximum error and AOAD in tandem allows for the successful characterization of a wide range of flight scenarios, whether for position hold or reference tracking, and of long or short duration. 


\section{Chapter 4}

\section{Control Methodologies}

Several control techniques are selected for the task of directing the position of the TARA quadrotor towards desired coordinates in simulation. Based on the literature review conducted in Chapter 2, the control methodologies investigated here are PID, integral backstepping (IB), fuzzy, and adaptive integral backstepping (AIB).

The ubiquitous presence of PID control in quadrotor platforms necessitates its inclusion in this study. In addition to PID, a specific focus is included on control techniques which are good in dealing with nonlinear systems subject to uncertainties and disturbances. Lyapunov stability based control techniques are represented here by IB, which is selected for its ability to keep the error bounded, as well as capacity for handling disturbances. As opposed to relying on a modeled system, fuzzy control uses an entirely different intelligent approach, and is able to deal with nonlinearities and uncertainties. Adaptive control, in the form of AIB, is chosen for its ability to change control parameters based on estimated disturbances.

For each of the included control techniques, its theory and the control law derivation for the quadrotor are presented. The controllers are then tuned in simulation in the absence of wind such that all four control techniques satisfy a good performance metric. This is done in order to observe the ability of each control technique in dealing with wind disturbances applied later. 


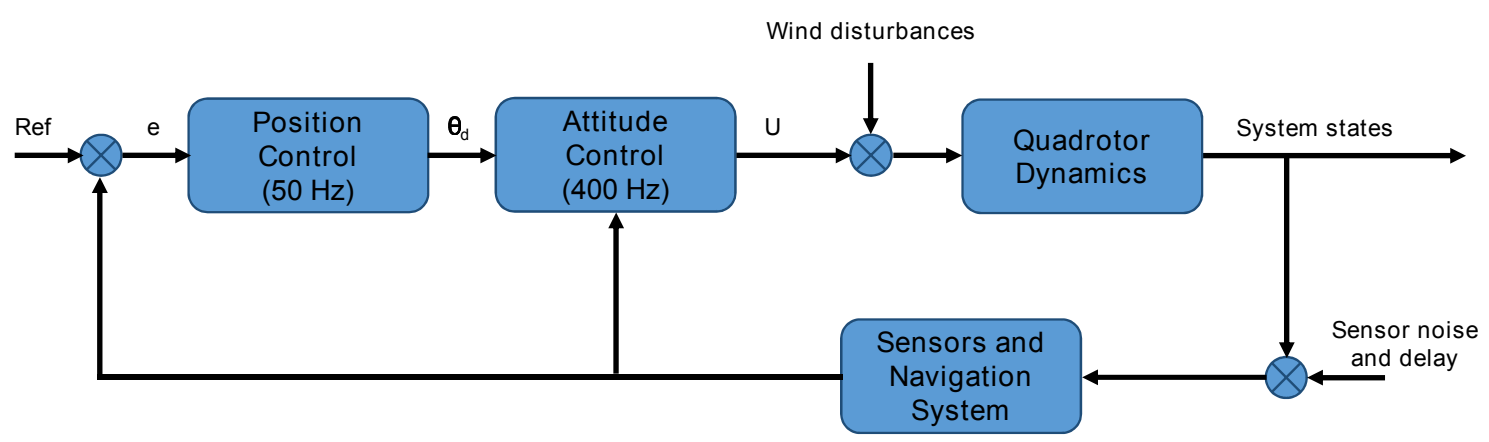

Figure 26: Flight control system architecture.

\subsection{PID control}

The main advantages of Proportional-Integral-Differential (PID) control lie in its relatively straightforward implementation and ability to produce effective control over a variety of applications. This control technique has been widely employed for the attitude stabilization and position control of quadrotors. Due to its prominent use in the flight control of quadrotors it is important to investigate the performance of PID control when subjected to urban wind conditions. Figure 26 illustrates a block diagram of the overall flight control architecture where attitude and position control are used in a cascade form.

\subsubsection{Attitude Stabilization}

Attitude stabilization at the low-level of the quadrotor autopilot is achieved by PID controllers (Equations 34, 35, and 36) for the pitch, roll and yaw angles, respectively. The PID control technique is carefully selected in accordance with the desired flight behaviour and performance expected from the quadrotor. TARA is designed to perform stable hovering, mostly keeping leveled pitch and roll angles, and slow position changes requiring small adjustments in desired attitude angles. This is contrary to 
some other designs which require a quadrotor to be a very agile and acrobatic maneuvering platform.

The gains for each PID controller are determined using the Simulink Design Optimization library in conjunction with the TARA flight simulator. A step response criteria for the desired attitude stabilization is established, such that for an initial angle of 60 degrees, the controller should have a rise time of 1.5 seconds for $80 \%$ rise, a maximum settling time of 3 seconds with $20 \%$ settling, an overshoot of less than $30 \%$, and an undershoot of $5 \%$. The tuned parameters are the PID controller gains $K_{P}$, $K_{I}$, and $K_{D}$, for pitch, roll, and yaw angles, respectively. A gradient descent based optimization algorithm is used for the determination of the appropriate PID gains to achieve the desired response. The final PID controller gains, shown in Table 3, are evaluated through the response optimization algorithm for the pitch, roll, and yaw axes respectively. The attitude control performance is evaluated by a performance metric, Table 4, similar to the position control. The performance grade ranges are defined in the light of numerous literature based experimental results.

$$
\begin{gathered}
U_{\theta}=K_{D_{\theta}}\left(\dot{\theta}_{r e f}-\dot{\theta}\right)+K_{P_{\theta}}\left(\theta_{r e f}-\theta\right)+K_{I_{\theta}} \int_{0}^{t}\left(\theta_{r e f}-\theta\right) d t \\
U_{\phi}=K_{D_{\phi}}\left(\dot{\phi}_{r e f}-\dot{\phi}\right)+K_{P_{\phi}}\left(\phi_{r e f}-\phi\right)+K_{I_{\phi}} \int_{0}^{t}\left(\phi_{r e f}-\phi\right) d t \\
U_{\psi}=K_{D_{\psi}}\left(\dot{\psi}_{r e f}-\dot{\psi}\right)+K_{P_{\psi}}\left(\psi_{r e f}-\psi\right)+K_{I_{\psi}} \int_{0}^{t}\left(\psi_{r e f}-\psi\right) d t
\end{gathered}
$$


Table 3: Finalized attitude controller gains.

\begin{tabular}{cccccccccc} 
Gains: & $K_{P_{\theta}}$ & $K_{D_{\theta}}$ & $K_{I_{\theta}}$ & $K_{P_{\phi}}$ & $K_{D_{\phi}}$ & $K_{I_{\phi}}$ & $K_{P_{\psi}}$ & $K_{D_{\psi}}$ & $K_{I_{\psi}}$ \\
\hline Value: & 11.0 & -20.0 & 0.02 & 11.0 & -20.0 & 0.02 & 11.0 & 15.0 & 0.5
\end{tabular}

Table 4: Attitude stabilization performance metric.

\begin{tabular}{|c|c|c|c|c|}
\hline Absolute Error $(\mathrm{deg})$ & $\leq 5$ & $5<$ error $\leq 10$ & $10<$ error $\leq 20$ & $>20$ \\
\hline \hline Performance Grade & Good & Acceptable & Poor & Bad \\
\hline
\end{tabular}

\subsubsection{Attitude Stabilization Results}

In order to evaluate the accuracy of the flight simulator and the performance of attitude controllers, an analysis is conducted whereby simulation flight data is compared with experimentally obtained flight data. Results for the attitude stabilization of TARA using the PID controller gains in Table 3 are presented. To maintain consistency in the test, a three minute long flight is simulated as well as experimentally conducted where TARA is commanded to takeoff and hover while performing position hold. As the test flight is performed indoors a no wind condition is used in the flight simulation.

Figure 27(a) depicts the flight simulation data obtained for pitch stabilization. Here the pitch angle is shown to remain consistently within the bounds of a good performance, as per the criteria established in Table 4, with a corresponding AOAD value of 1.07 degrees. The experimental results, presented in Figure 27(b), also remain mostly within this \pm 5 degree envelope, with a few limited exceptions. The overall consistency of the results is reflected in the 1.74 degree AOAD for the experimental flight, which is comparable to the simulation value.

Similarly, the simulation roll angle stabilization results depicted in Figure 28(a) vary within the \pm 5 degree limits established as characterizing a good performance. The simulation data evidences an AOAD of 1.19 degrees. For the experimental flight, 


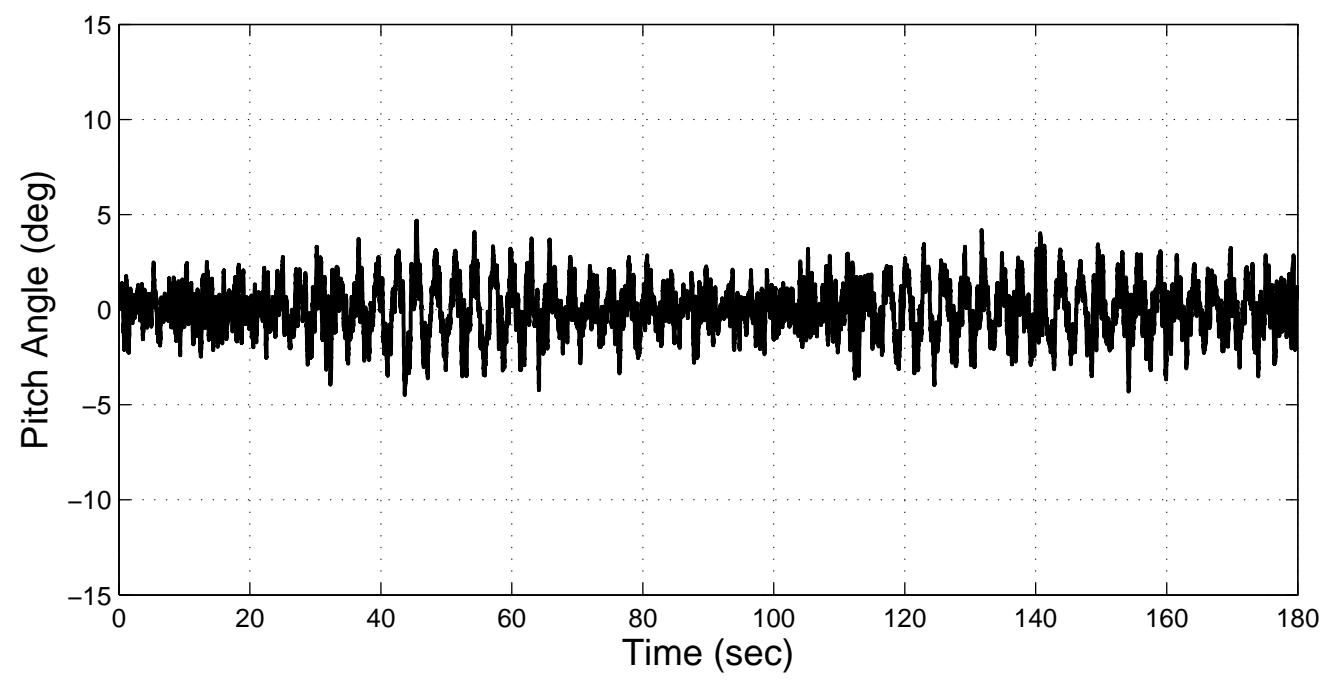

(a) Simulation $\left(\mathrm{AOAD}=1.07^{\circ}\right)$

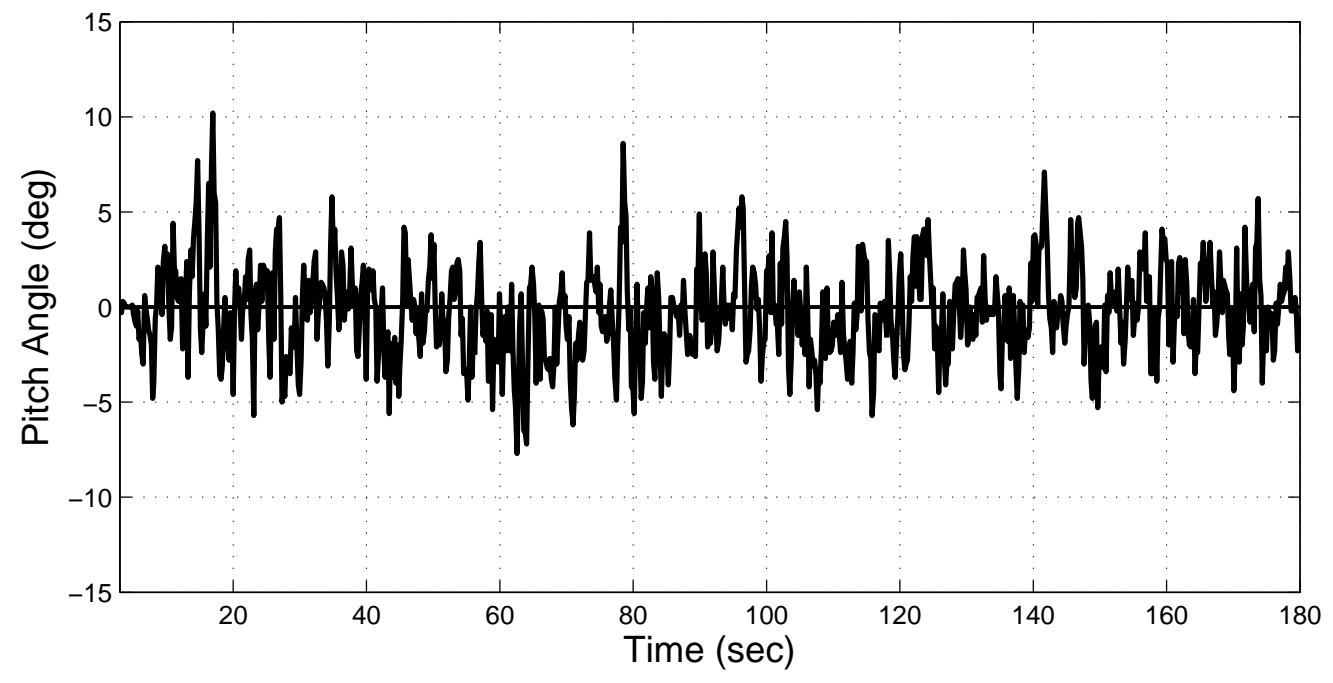

(b) Experimental $\left(\mathrm{AOAD}=1.74^{\circ}\right)$

Figure 27: Pitch angle flight data. 
shown in Figure 28(b), a corresponding AOAD of 1.43 degrees is obtained.

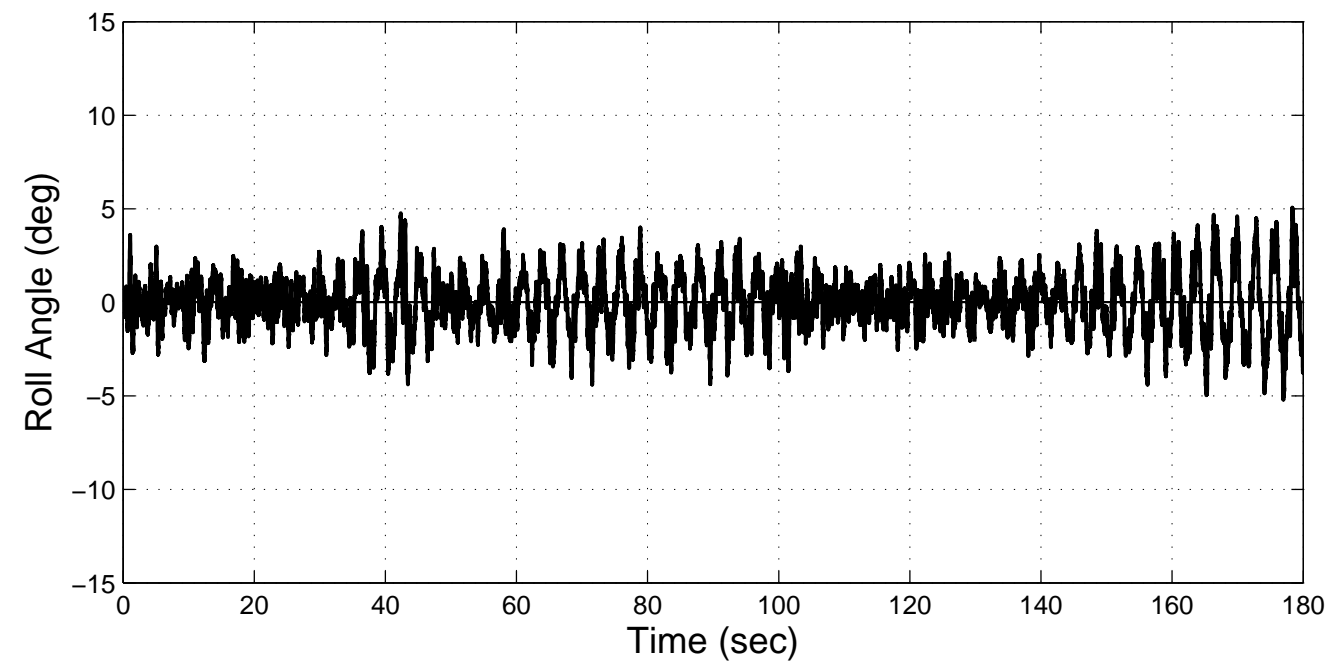

(a) Simulation $\left(\mathrm{AOAD}=1.19^{\circ}\right)$

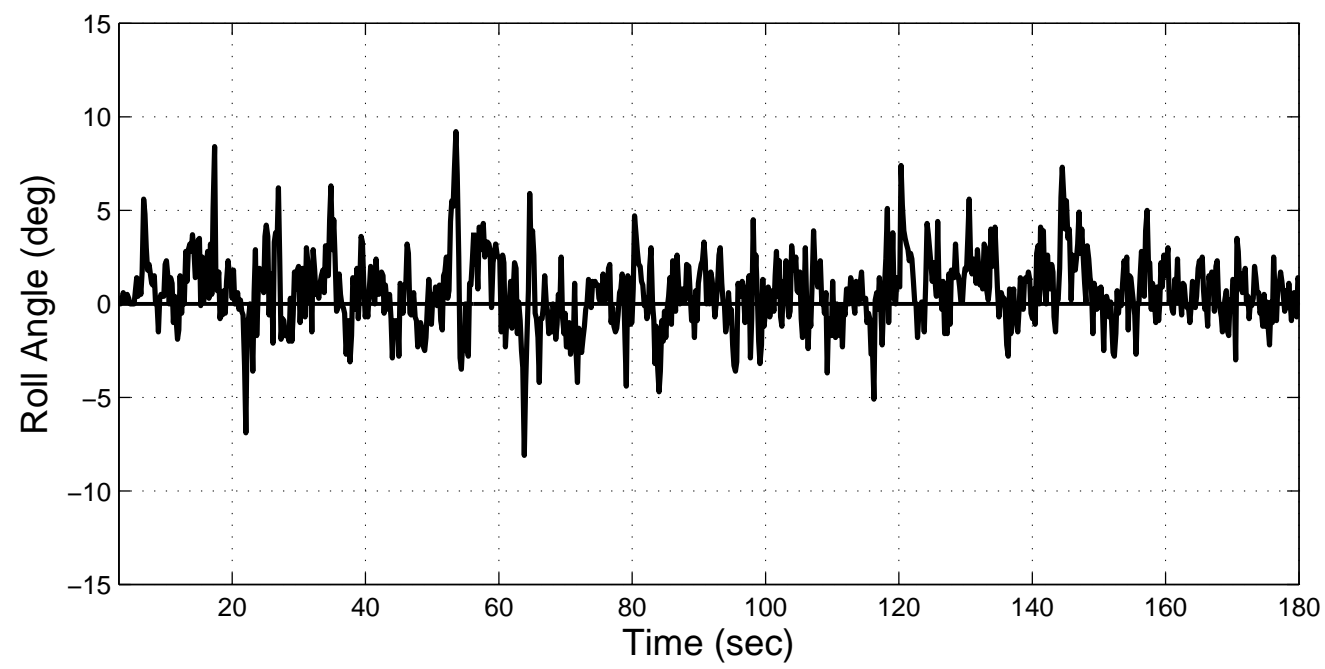

(b) Experimental $\left(\mathrm{AOAD}=1.43^{\circ}\right)$

Figure 28: Roll angle flight data.

For the yaw angle stabilization, the desired yaw reference is -85 degrees which is TARA's heading recorded from the on-board compass at the time of take-off. The simulation and experimental flight results for yaw angle stabilization can be viewed in Figure 29. In comparison with the pitch and roll values, greater variability is evident in the yaw angle. While the 4.01 degree AOAD obtained for the experimental data 
is still within the established good metric, the simulation yielded a much better AOAD of 0.83 degrees. This higher AOAD in experimental data for yaw angle is mostly attributed to minor differences in propulsion units resulting in yaw imbalances, especially when fast changes in altitude occur. The designed yaw control is successful in stabilizing these disturbances. However, with the drawback of oscillations with higher peaks. Another source of yaw deviations is the EMI generated from the power lines. At times when TARA requires higher thrust from all motors the total current drawn ranges from 40 to $60 \mathrm{~A}$. This increased current causes large EMI noise which results a in yaw heading bias of 15 degrees. Yaw control performance is degraded in such cases.

\subsubsection{PID Position Control}

In order to achieve a stable position hold a PD control scheme is implemented for the $\mathrm{X}$ and $\mathrm{Y}$-axis position control, and a PID control scheme is used for the Z-axis altitude control, per Equations 37, 38, and 39, respectively. Table 5 lists the gain values used for position control.

The gain tuning process in the Simulink Design Optimization library is similar to the steps defined earlier in the attitude control section. A step response criteria for the desired position control is established, where the altitude control is given higher priority in terms of faster rise and settling time. This is done to minimize the influence of altitude control efforts on the lateral $\mathrm{X}$-axis and $\mathrm{Y}$-axis control performances. Thus, for a $10 \mathrm{~m}$ step input, the controller should have a rise time of 5 seconds for altitude control, and 20 seconds for lateral control. Similarly, the settling time allocated for the altitude control is 15 seconds, and for lateral control is 30 seconds. The desired \% settling is $10 \%$. The overshoot criteria for altitude control is relaxed to $30 \%$ in order to allow for a faster rise time. However, the lateral control is designed to produce a minimal overshoot of $10 \%$. The undershoot criteria is set to $5 \%$ for both altitude and 


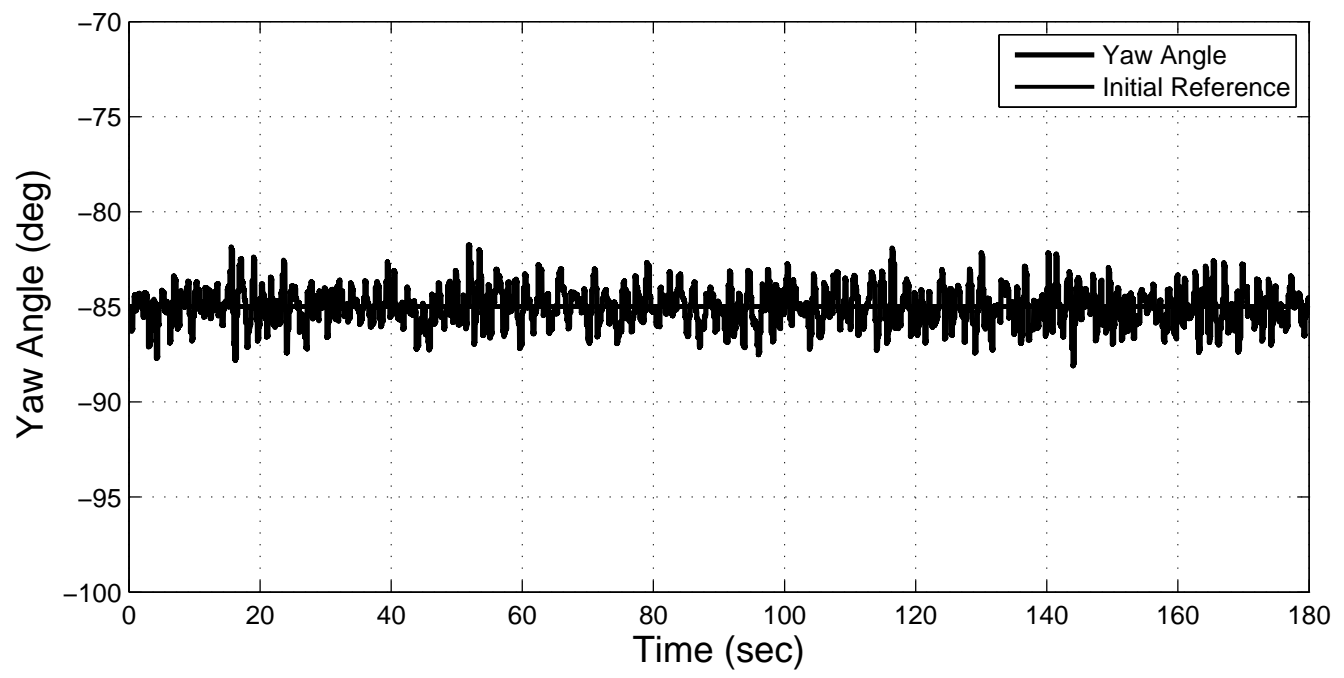

(a) Simulation $\left(\mathrm{AOAD}=0.83^{\circ}\right)$

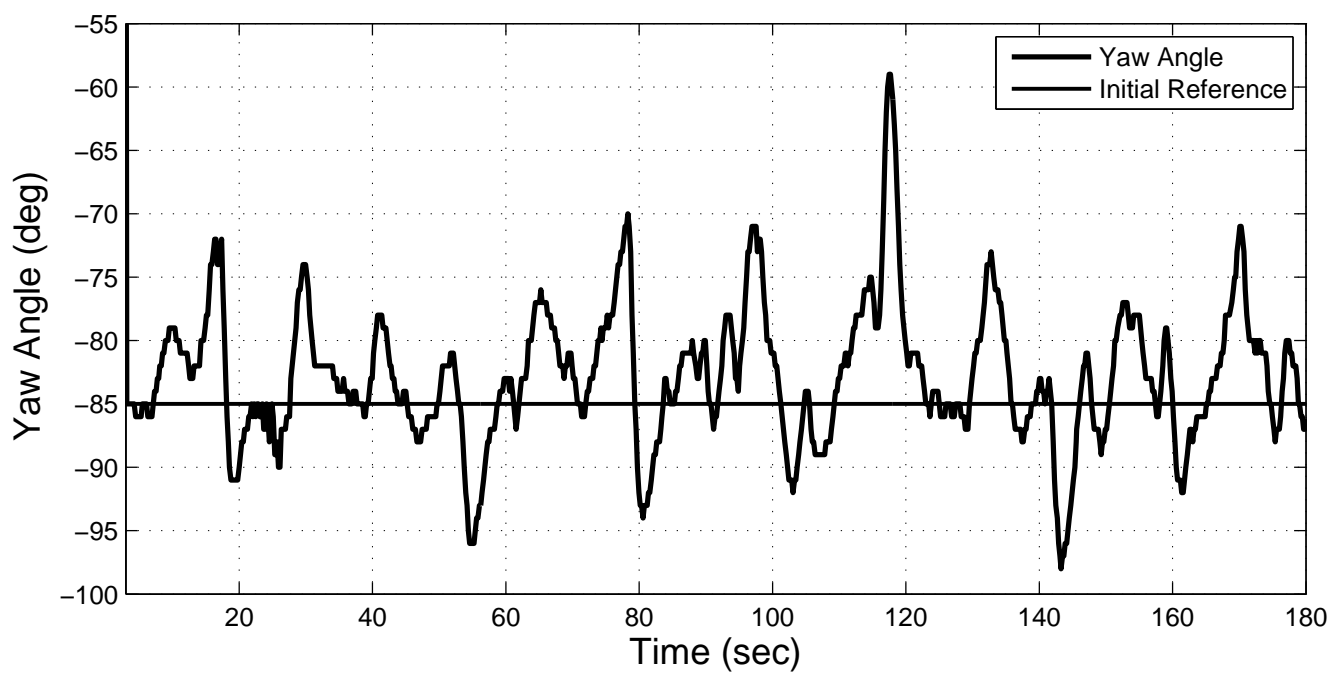

(b) Experimental $\left(\mathrm{AOAD}=4.01^{\circ}\right)$

Figure 29: Yaw angle flight data. 
lateral controls. The criteria specified here is also employed in tuning the gains for the IB and AIB controllers.

$$
\begin{gathered}
U_{X}=K_{D_{X}}\left(\dot{X}_{r e f}-\dot{X}\right)+K_{P_{X}}\left(X_{r e f}-X\right) \\
U_{Y}=K_{D_{Y}}\left(\dot{Y}_{r e f}-\dot{Y}\right)+K_{P_{Y}}\left(Y_{r e f}-Y\right) \\
U_{Z}=K_{D_{Z}}\left(\dot{Z}_{r e f}-\dot{Z}\right)+K_{P_{Z}}\left(Z_{r e f}-Z\right)+K_{I_{Z}} \int_{0}^{t}\left(Z_{r e f}-Z\right) d t
\end{gathered}
$$

Table 5: PID position controller gains.

\begin{tabular}{lrrrrrrr} 
Gains: & $K_{D_{X}}$ & $K_{P_{X}}$ & $K_{D_{Y}}$ & $K_{P_{Y}}$ & $K_{D_{Z}}$ & $K_{P_{Z}}$ & $K_{I_{Z}}$ \\
\hline Value: & 4.0 & 0.2 & 4.0 & 0.2 & 1.0 & 0.4 & 0.05
\end{tabular}

\subsection{Integral Backstepping Position Control}

Backstepping is a stabilizing control technique for a special class of nonlinear systems that are comprised of subsystems expanding out from an irreducible subsystem which can be stabilized using some control technique. A recursive application of backstepping allows for the stabilization of systems that are in strict feedback form. The systematic backstepping procedure starts with the origin subsystem with stability criteria and steps backward while maintaining stability at each step ensuring global asymptotic stability of the system. In particular, backstepping control is advantageous in scenarios where a system state is controlled through the action of another state, as is the case with the quadrotor, where lateral motion is achieved by varying the pitch and roll angles. Furthermore, compared with feedback linearization techniques, a trademark of backstepping is the flexibility it offers, in that it can avoid 
the cancellation of useful nonlinearities and may produce a control law requiring less control effort.

This section presents the position controller design using the backstepping control technique. The position controller's stability is guaranteed by applying Lyapunov's stability theory in the design. The primary advantage of using backstepping control is its robustness against disturbances. When combined with an integral action, which offers robustness against model uncertainties, the resultant integral backstepping control provides improved control performance.

\subsubsection{Lyapunov Stability Theory}

This stability theory for dynamic systems was developed by the Russian mathematician Aleksandr Lyapunov. Named after him, Lyapunov theory is employed in order to characterize the local stability of equilibrium points, as well as the overall stability properties of a dynamic system based on a type of generalized energy function, without actually solving the system differential equations. Based on the logic that if a function is always decreasing then it must eventually reach zero, hence as a system it may never diverge and is therefore stable in nature. Before proceeding, we may

consider the following relevant stability concept definitions. A function $V(x)$ is said to be:

- positive definite if $V(0)=0$ and $V(x)>0$ with $x \neq 0$.

- positive semidefinite if $V(0)=0$ and $V(x) \geq 0$ with $x \neq 0$.

- negative semidefinite if $-V(x)$ is positive semidefinite.

- radially unbounded if $V(x) \rightarrow \infty$ as $|x| \rightarrow \infty$ 
Let us now consider a time-invariant system with state $x$ :

$$
\dot{x}=f(x)
$$

where $x(t)$ is a function with initial state $x(0)=x_{o}$.

To prove that this nonlinear system is stable, the second or direct method of Lyapunov is utilized. Lyapunov's direct method characterizes the dynamic behavior of the system by using an appropriate candidate function. This function $V(x)$, has to be positive definite in a region near $x=0$, and is referred to as a Lyapunov function. A useful analogy is to think of this function as being energy-like, such that if the energy in the system can be measured then the rate of change of that energy can help in studying the stability of the system.

Therefore the time derivative of $V(x)$ is:

$$
\dot{V}(x)=\frac{\partial V(x)}{\partial t}=\frac{\partial V(x)}{\partial x} \frac{\partial x}{\partial t}=\frac{\partial V(x)}{\partial x} F(x)
$$

Lyapunov theory states that

- if $\dot{V}(x)$ is negative semidefinite for a given region, then the solution is stable.

- if $\dot{V}(x)$ is negative definite for a given region, then the solution is asymptotically stable.

- if $V(x)$ is positive definite and radially unbounded for all $x$, and if $\dot{V}(x)$ negative definite for all $x$, then the solution is globally asymptotically stable.

Now let us define these stable systems in sense of Lyapunov:

- Stable if, for given $\epsilon>0$, there exists a $\delta(\epsilon)>0$ such that all solutions with initial conditions $|x(0)|<\delta$ satisfy $|x(t)|<\epsilon$ for all $t \geq 0$. All solutions starting near $x=0$ remain bounded. 
- Asymptotically stable if it is stable and a $\delta$ can be found such that all solutions with $|x(0)|<\delta$ satisfy $|x(t)| \rightarrow 0$ as $t \rightarrow \infty$. All solutions starting near $x=0$ are bounded and converge to zero.

- Globally asymptotically stable if it is asymptotically stable for any initial $x(0)$ state.

In order to achieve a closed-loop system with the desirable stability a control law needs to be derived which involves using a suitable control Lyapunov function (clf). For most nonlinear systems the clf is not known and finding an appropriate clf is a complex task. However, if the system is scalar, a quadratic clf $V(x)=\frac{1}{2} x^{2}$, is a reasonable starting point.

\subsubsection{Backstepping Control Technique}

In order to understand the recursive design procedure which forms the basis of the backstepping control technique, let us consider a nonlinear system model of strictfeedback form [139]:

$$
\begin{aligned}
& \dot{x}=f(x)+g(x) \xi_{1} \\
& \dot{\xi}_{1}=f_{1}\left(x, \xi_{1}\right)+g_{1}\left(x, \xi_{1}\right) \xi_{2} \\
& \dot{\xi}_{2}=f_{2}\left(x, \xi_{1}, \xi_{2}\right)+g_{2}\left(x, \xi_{1}, \xi_{2}\right) \xi_{3} \\
& \cdot \\
& \cdot \\
& \dot{\xi}_{k}=f_{k}\left(x, \xi_{1}, \ldots \xi_{k}\right)+g_{k}\left(x, \xi_{1}, \ldots \xi_{k}\right) u
\end{aligned}
$$


where $x \in \Re^{n}$ and $\xi_{1}, . . \xi_{k}$ are scalars. Input $u$ is the control signal, through which our goal is to direct $x$ towards some desired value. Here strict-feedback refers to the fact that the nonlinearities $f_{i}, g_{i}$ depend solely on state variables $x, \xi_{1}, \ldots, \xi_{i}$ which have been fed back into the system.

For simplicity, consider a system with $k=2$ :

$$
\begin{aligned}
& \dot{x}=f(x)+g(x) \xi_{1} \\
& \dot{\xi}_{1}=u
\end{aligned}
$$

at $x=0$ with $f(0)=0$.

Figure 30(a) depicts this initial system in the form of a block diagram. Our objective is to stabilize the origin of the system represented by Equation 43 with $u$. Consider the first component subsystem, which is stabilized by input $\xi_{1}$. Per Lyapunov theory, let us assume we can introduce a feedback controller $\xi_{1}=\phi(x)$ with $\phi(0)=0$, as well as a candidate Lyapunov function, such as $V(x)=\frac{1}{2} x^{2}$, which satisfies the stability criteria:

$$
\frac{\partial V}{\partial x}[f(x)+g(x) \phi(x)] \leq-W(x)
$$

where $W(x)$ is positive definite. Adding and subtracting $g(x) \phi(x)$ to Equation 43 yields the system shown in Figure 30(b), where $\dot{x}=[f(x)+g(x) \phi(x)]+g(x)\left[\xi_{1}-\phi(x)\right]$. However, it is important to note that $\xi_{1}$ is not our control input, and we cannot control it directly in order to achieve stability. Hence, $\xi_{1}$ is referred to as a virtual control and $\phi(x)$ as the stabilizing function. The deviation of this virtual control in tracking its desired value $\phi(x)$ can be defined as the error signal $e_{1}=\xi_{1}-\phi(x)$. Since $\dot{e}_{1}=\dot{\xi}_{1}-\dot{\phi}(x)$, the control $\phi(x)$ can be said to have been "backstepped" through the integrator, as depicted in Figure 30(c). Defining a new variable, $w=u-\dot{\phi}(x)$, we 


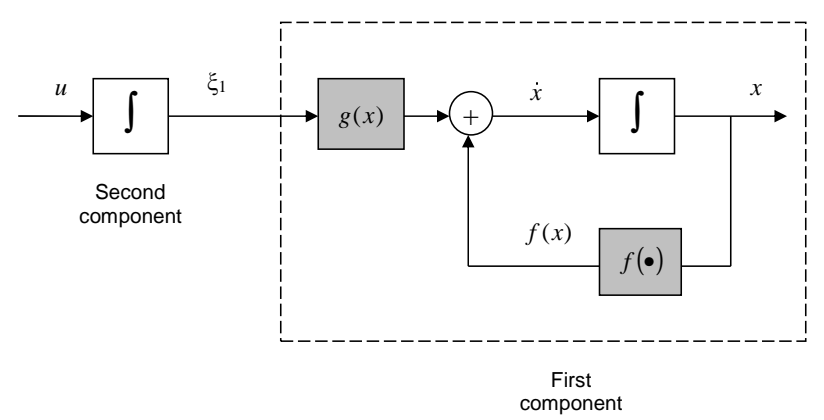

(a)

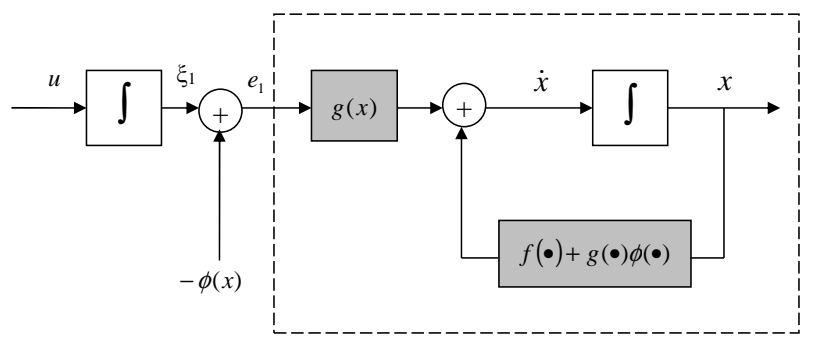

(b)

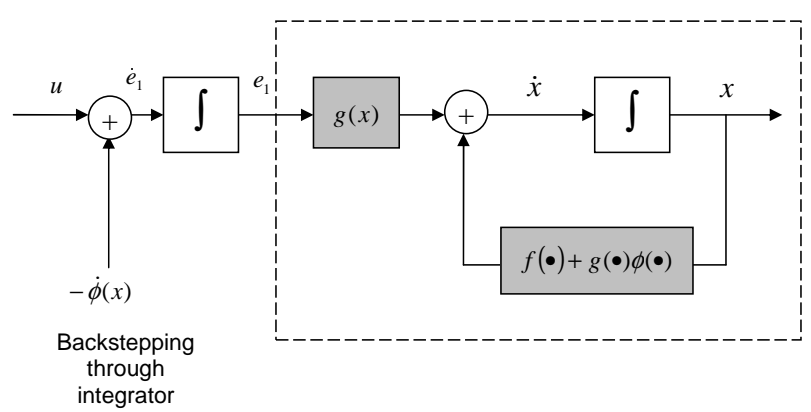

(c)

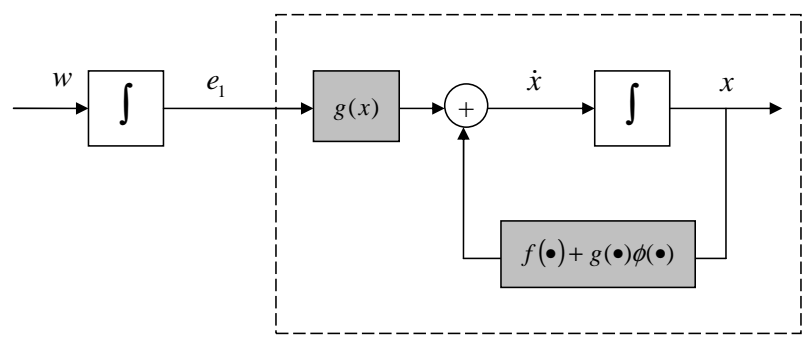

(d)

Figure 30: The backstepping control. (a) The initial system block diagram. (b) Introducing feedback controller. (c) Backstepping control through the integrator. (d) Final system block diagram. 
can rewrite our system to the block diagram shown in Figure 30(d), where:

$$
\begin{aligned}
& \dot{x}=[f(x)+g(x) \phi(x)]+g(x) e_{1} \\
& \dot{e}_{1}=w
\end{aligned}
$$

This final system has a similar structure as the initial system, except that now the first component, as shown in Figure 30(a) is asymptotically stable at the origin. In

order to stabilize the overall system, we can appropriately augment our candidate Lyapunov function with $e_{1}$ and choose a stabilizing control law for $u$ to ensure that this augmented Lyapunov function is negative definite, stabilizing the origin globally and asymptotically. For systems with $k>2$ this recursive design procedure, defining virtual controls and error signals, can be followed for as many iterations as required to step back to the origin.

In the presence of uncertainty, backstepping provides a systematic procedure for nonlinear design. Systems in which the uncertainty appears in the same equation as the control are said to have "matched" uncertainty. In this case, augmenting the control law with a nonlinear damping term can be used to guarantee global boundedness and convergence, even though the bound on the disturbance is unknown. Furthermore, even when the matching condition is not satisfied and the uncertainties do not appear within the span of the control input, a static nonlinear controller combining backstepping and nonlinear damping can be designed which guarantees global uniform boundedness.

\subsubsection{Integral Backstepping Control Law Synthesis}

Position control is achieved using integral backstepping controllers [31] for the X, Y, and $\mathrm{Z}$ axes, respectively. The synthesis of the control laws for a quadrotor is presented in the following sub-sections. 


\subsubsection{Simplified Model for Control Design}

For autonomous flight control design purposes the body frame equations of motion are transformed to the earth frame of reference. A simplified model is adopted such that a near hover condition is assumed during flight. High velocity translational effects are also ignored based on the fact that TARA is designed for slow position changes.

The following assumptions are made:

- small $\phi$ and $\theta$ angle approximations during hover flight are used to reduce the transformation matrix in Equation 16 between rate of change of the orientation angles $\left[\begin{array}{lll}\dot{\phi} & \dot{\theta} & \dot{\psi}\end{array}\right]^{T}$ and the body angular velocities $\left[\begin{array}{lll}p & q & r\end{array}\right]^{T}$ to unity matrix. Thus, $\left[\begin{array}{lll}\dot{\phi} & \dot{\theta} & \dot{\psi}\end{array}\right]^{T} \approx\left[\begin{array}{lll}p & q & r\end{array}\right]^{T}$.

- neglecting the Coriolis terms $q r$, $p r$, and $p q$, as angular rates are kept small during hover and translational motion, will eliminate the first matrix in Equation 30 .

Therefore, the simplified rotational model for attitude control design is:

$$
\left[\begin{array}{c}
\ddot{\phi} \\
\ddot{\theta} \\
\ddot{\psi}
\end{array}\right]=\left[\begin{array}{c}
\frac{1}{j_{x}} l\left(T_{l}-T_{r}\right) \\
\frac{1}{j_{y}} l\left(T_{f}-T_{b}\right) \\
\frac{1}{j_{z}}\left(\tau_{r}+\tau_{l}-\tau_{f}-\tau_{b}\right)
\end{array}\right]
$$

Similarly, the translational model is simplified by making the following assumptions:

- differentiating Equation 17 and neglecting the derivative of body to earth rotation matrix $\dot{R}$ results in $\left[\begin{array}{lll}\ddot{X} & \ddot{Y} & \ddot{Z}\end{array}\right]^{T}=R\left[\begin{array}{lll}\dot{u} & \dot{v} & \dot{w}\end{array}\right]^{T}$ for small angular rates, which implies that the body frame accelerations can be transformed to the earth frame via $R$. 
- neglecting the Coriolis terms in Equation 29.

- the aerodynamic forces $F_{\text {aero }}$ in Equation 29 are not included in the simplified model, as wind effects take the form of unknown disturbances in control design.

Using these assumptions the simplified translational model for position control design is:

$$
\left[\begin{array}{c}
\ddot{X} \\
\ddot{Y} \\
\ddot{Z}
\end{array}\right]=\left[\begin{array}{c}
0 \\
0 \\
g
\end{array}\right]+\frac{1}{M} R\left[\begin{array}{c}
0 \\
0 \\
-T
\end{array}\right]
$$

and multiplying by $\mathrm{R}$ gives:

$$
\left[\begin{array}{c}
\ddot{X} \\
\ddot{Y} \\
\ddot{Z}
\end{array}\right]=\left[\begin{array}{l}
0 \\
0 \\
g
\end{array}\right]+\frac{1}{M}\left[\begin{array}{c}
-T(\sin \theta \cos \phi \cos \psi+\sin \phi \sin \psi) \\
-T(\sin \theta \cos \phi \sin \psi-\sin \phi \cos \psi) \\
-T \cos \theta \cos \phi
\end{array}\right]
$$

\subsubsection{Integral Backstepping Altitude Control}

The simplified system equation for Z-axis motion in the earth frame is (from Equation 48),

$$
\ddot{Z}=g-\frac{\cos (\theta) \cos (\phi) U_{Z}}{M}
$$

where $U_{Z}$ is the control input representing the cumulative motor thrust necessary to modify the altitude as required (i.e. $U_{Z}=T$ in Equation 48).

The first step in the design of integral backstepping control for $U_{Z}$ is to define the tracking error, $e_{Z}$, as the difference between the desired and actual Z-axis position,

$$
e_{Z}=Z_{r e f}-Z
$$


The error rate is also evaluated by taking the time derivative of the tracking error,

$$
\dot{e}_{Z}=\frac{d e_{Z}}{d t}=\dot{Z}_{r e f}-\dot{Z}
$$

A virtual control input, $v_{Z_{1}}$, is set as a desired behavior for $\dot{Z}$, such that the stabilizing function is composed of terms which are proportional to the tracking error, $e_{Z}$, its integral, and a term which accounts for a change in the desired altitude,

$$
v_{Z_{1}}=c_{5} e_{Z}+\dot{Z}_{r e f}+\lambda_{3} \chi_{3}
$$

where $c_{5}$ and $\lambda_{3}$ are positive constants, and $\chi_{3}=\int e_{Z}(\Delta t) \partial \Delta t$.

The effectiveness of $\dot{Z}$ in following $v_{Z_{1}}$ can be measured by its own tracking error, $e_{v_{Z_{1}}}$,

$$
e_{v_{Z_{1}}}=v_{Z_{1}}-\dot{Z}=c_{5} e_{Z}+\dot{Z}_{r e f}+\lambda_{3} \chi_{3}-\dot{Z}
$$

The rate at which this error changes with time can be found as,

$$
\dot{e}_{v_{Z_{1}}}=\frac{d e_{v_{Z_{1}}}}{d t}=c_{5} \dot{e}_{Z}+\ddot{Z}_{r e f}+\lambda_{3} e_{Z}-\ddot{Z}
$$

Noting that $\dot{e}_{Z}=\dot{Z}_{r e f}-\dot{Z}$ one can re-write Equation 54 as,

$$
\dot{e}_{v_{Z_{1}}}=\frac{d e_{v_{Z_{1}}}}{d t}=c_{5}\left(\dot{Z}_{r e f}-\dot{Z}\right)+\ddot{Z}_{r e f}+\lambda_{3} e_{Z}-\ddot{Z}
$$

while also noting that Equation 53 can be re-arranged to obtain,

$$
\dot{Z}_{r e f}-\dot{Z}=e_{v_{Z_{1}}}-c_{5} e_{Z}-\lambda_{3} \chi_{3}
$$


yields the rate of change in the error of the virtual control,

$$
\dot{e}_{v_{Z_{1}}}=\frac{d e_{v_{Z_{1}}}}{d t}=c_{5}\left(e_{{v_{1}}_{1}}-c_{5} e_{Z}-\lambda_{3} \chi_{3}\right)+\ddot{Z}_{r e f}+\lambda_{3} e_{Z}-\left(g-\frac{U_{Z} \cos (\theta) \cos (\phi)}{M}\right)
$$

where the acceleration in the $\mathrm{Z}$ direction has been replaced by the aircraft dynamics as governed by Equation 49 .

At this point another virtual controller can be introduced, $v_{Z_{2}}$, where the action of $v_{Z_{2}}$ is that it tries to follow the rate of change of $e_{v_{Z_{1}}}$,

$$
v_{Z_{2}} \equiv \dot{e}_{v_{Z_{1}}}
$$

where $v_{Z_{2}}$ is proportional to both the error in $v_{Z}$ and the original tracking error, $e_{Z}$,

$$
v_{Z_{2}}=-c_{6} e_{{Z_{1}}_{1}}-e_{Z}
$$

Thought of another way, from Equation 53 we can write,

$$
v_{Z_{2}}=\dot{e}_{v_{Z_{1}}}=\dot{v}_{Z_{1}}-\ddot{Z}
$$

Thus, $v_{Z_{2}}$ represents the error between how well the rate of change of the first virtual controller follows the true value of $\ddot{Z}$.

Noting that we already have an expression for $\dot{e}_{v_{Z_{1}}}$ in Equation 57 allows us to write,

$$
-c_{6} e_{v_{Z_{1}}}-e_{Z}=c_{5}\left(e_{v_{Z_{1}}}-c_{5} e_{Z}-\lambda_{3} \chi_{3}\right)+\ddot{Z}_{r e f}+\lambda_{3} e_{Z}-\left(g-\frac{U_{Z} \cos (\theta) \cos (\phi)}{M}\right)
$$

Finally, we can isolate for the original control function:

$$
U_{Z}=\frac{M}{\cos (\theta) \cos (\phi)}\left[g+\left(c_{5}+c_{6}\right) e_{{Z_{1}}_{1}}+\left(1-c_{5}^{2}+\lambda_{3}\right) e_{Z}-c_{5} \lambda_{3} \chi_{3}+\ddot{Z}_{r e f}\right]
$$


where $c_{5}, c_{6}, \lambda_{3}$ are all positive constants. The values for these constants are listed in Table 6, as determined in simulation using the Design Optimization library in Simulink.

\subsubsection{Stability Analysis of Altitude Control Law}

Selecting a suitable positive definite control Lyapunov function, $V$, for the system,

$$
V=\frac{1}{2}\left[e_{Z}^{2}+e_{v_{Z_{1}}}^{2}+\lambda_{3} \chi_{3}^{2}\right]
$$

The derivative of this Lyapunov function is,

$$
\begin{gathered}
\dot{V}=\frac{1}{2}\left[2 e_{Z}\left(e_{v_{Z_{1}}}-c_{5} e_{Z}-\lambda_{3} \chi_{3}\right)+2 e_{v_{Z_{1}}}\left(-c_{6} e_{v_{Z_{1}}}-e_{Z}\right)+2 \lambda_{3} \chi_{3} e_{Z}\right] \\
\dot{V}=e_{Z} e_{v_{Z_{1}}}-c_{5} e_{Z}^{2}-\lambda_{3} \chi_{3} e_{Z}-c_{6} e_{v_{Z_{1}}}^{2}-e_{v_{Z_{1}}} e_{Z}+\lambda_{3} \chi_{3} e_{Z} \\
\dot{V}=-c_{5} e_{Z}^{2}-c_{6} e_{v_{Z_{1}}}^{2} \leq 0
\end{gathered}
$$

The negative semi-definite $\dot{V}$ guarantees the stability of the altitude control law.

\subsubsection{Integral Backstepping Lateral Position Control}

The lateral position of the quadrotor in the $\mathrm{X}$ and $\mathrm{Y}$ axes may also be regulated by integral backstepping control laws. The synthesis of X-axis and Y-axis position control laws are provided together due to their similar nature.

Assuming near hover flight conditions, the small angle approximation may be applied, such that the simplified system equations for $\mathrm{X}$ and $\mathrm{Y}$-axis motion (from 
Equation 48) become:

$$
\begin{gathered}
\ddot{X}=\frac{\left(U_{X}\right)}{M} T \\
\ddot{Y}=\frac{\left(-U_{Y}\right)}{M} T
\end{gathered}
$$

where $U_{X}=\theta_{d}$ and $-U_{Y}=\phi_{d}$ are the $\mathrm{X}$ and $\mathrm{Y}$-axis position controller outputs in the form of desired pitch and roll angles, respectively. These desired pitch and roll angles become the reference input for the attitude controllers. The objective in setting these desired angles is to direct the overall quadrotor thrust in the direction of desired $\mathrm{X}$ and Y position coordinates.

Tracking errors $e_{X}$ and $e_{Y}$ are designated as the difference between the desired and actual $\mathrm{X}$ and $\mathrm{Y}$-axis positions,

$$
\begin{gathered}
e_{X}=X_{r e f}-X \\
e_{Y}=Y_{\text {ref }}-Y
\end{gathered}
$$

The error rates are evaluated by taking the time derivative of the tracking errors:

$$
\begin{gathered}
\dot{e}_{X}=\frac{d e_{X}}{d t}=\dot{X}_{r e f}-\dot{X} \\
\dot{e}_{Y}=\frac{d e_{Y}}{d t}=\dot{Y}_{r e f}-\dot{Y}
\end{gathered}
$$

The virtual control inputs $v_{X_{1}}$ and $v_{Y_{1}}$ are set as desired behaviors such that they try to follow $\dot{X}$ and $\dot{Y}$, respectively and similar to $v_{Z_{1}}$ are composed of terms which are proportional to their respective tracking error and its derivative, as well as a term 
which accounts for a change in the desired position for the corresponding axis.

$$
\begin{gathered}
v_{X_{1}}=c_{1} e_{X}+\dot{X}_{r e f}+\lambda_{1} \chi_{1} \\
v_{Y_{1}}=c_{3} e_{Y}+\dot{Y}_{r e f}+\lambda_{2} \chi_{2}
\end{gathered}
$$

where $\chi_{1}=\int e_{X}(\Delta t) \partial \Delta t$ and $\chi_{2}=\int e_{Y}(\Delta t) \partial \Delta t$. Also, $c_{1}, c_{3}, \lambda_{1}$ and $\lambda_{2}$ are all positive constants.

The tracking errors $e_{v_{X_{1}}}$ and $e_{v_{Y_{1}}}$ measure the effectiveness of the virtual control inputs,

$$
\begin{gathered}
e_{v_{X_{1}}}=v_{X_{1}}-\dot{X}=c_{1} e_{X}+\dot{X}_{r e f}+\lambda_{1} \chi_{1}-\dot{X} \\
e_{v_{Y_{1}}}=v_{Y_{1}}-\dot{Y}=c_{3} e_{Y}+\dot{Y}_{r e f}+\lambda_{2} \chi_{2}-\dot{Y}
\end{gathered}
$$

which can be re-arranged to obtain,

$$
\begin{gathered}
\dot{X}_{r e f}-\dot{X}=e_{v_{X_{1}}}-c_{1} e_{X}-\lambda_{1} \chi_{1} \\
\dot{Y}_{r e f}-\dot{Y}=e_{v_{Y_{1}}}-c_{3} e_{Y}-\lambda_{2} \chi_{2}
\end{gathered}
$$

The rate at which $e_{v_{X_{1}}}$ and $e_{v_{Y_{1}}}$ change with time is,

$$
\begin{gathered}
\dot{e}_{v_{X_{1}}}=\frac{d e_{v_{X_{1}}}}{d t}=c_{1} \dot{e}_{X}+\ddot{X}_{r e f}+\lambda_{1} e_{X}-\ddot{X}=c_{1}\left(\dot{X}_{r e f}-\dot{X}\right)+\ddot{X}_{r e f}+\lambda_{1} e_{X}-\ddot{X} \\
\dot{e}_{v_{Y_{1}}}=\frac{d e_{v_{Y_{1}}}}{d t}=c_{3} \dot{e}_{Y}+\ddot{Y}_{r e f}+\lambda_{2} e_{Y}-\ddot{Y}=c_{3}\left(\dot{Y}_{r e f}-\dot{Y}\right)+\ddot{Y}_{r e f}+\lambda_{2} e_{Y}-\ddot{Y}
\end{gathered}
$$


where $\ddot{X}_{\text {ref }}$ and $\ddot{Y}_{\text {ref }}$ are the desired accelerations.

Substituting relations in Equations 79 and 80, along with the quadrotor dynamics as prescribed by Equations 67 and 68, we obtain,

$$
\begin{gathered}
\dot{e}_{v_{X_{1}}}=\frac{d e_{v_{X_{1}}}}{d t}=c_{1}\left(e_{v_{X_{1}}}-c_{1} e_{X}-\lambda_{1} \chi_{1}\right)+\ddot{X}_{r e f}+\lambda_{1} e_{X}-\frac{\left(U_{X}\right)}{M} T \\
\dot{e}_{v_{Y_{1}}}=\frac{d e_{v_{Y_{1}}}}{d t}=c_{3}\left(e_{v_{Y_{1}}}-c_{3} e_{Y}-\lambda_{2} \chi_{2}\right)+\ddot{Y}_{r e f}+\lambda_{2} e_{Y}+\frac{\left(U_{Y}\right)}{M} T
\end{gathered}
$$

Introducing further virtual controllers, $v_{X_{2}}=\dot{e}_{v_{X_{1}}}$ and $v_{Y_{2}}=\dot{e}_{v_{Y_{1}}}=$, such that,

$$
\begin{gathered}
v_{X_{2}}=\frac{d e_{v_{X_{1}}}}{d t}=-c_{2} e_{v_{X_{1}}}-e_{X} \\
v_{Y_{2}}=\frac{d e_{{v_{1}}_{1}}}{d t}=-c_{4} e_{v_{Y_{1}}}-e_{Y}
\end{gathered}
$$

Substituting for $\dot{e}_{v_{X_{1}}}$ and $\dot{e}_{v_{Y_{1}}}$ in Equations 81 and 82 , we can evaluate the X-axis and Y-axis control inputs $U_{X}$ and $U_{Y}$, yielding the control laws,

$$
\begin{aligned}
& U_{X}=\frac{M}{T}\left[\left(1-c_{1}^{2}+\lambda_{1}\right) e_{X}+\left(c_{1}+c_{2}\right) e_{v_{X}}-c_{1} \lambda_{1} \chi_{1}+\ddot{X}_{r e f}\right] \\
& U_{Y}=-\frac{M}{T}\left[\left(1-c_{3}^{2}+\lambda_{2}\right) e_{Y}+\left(c_{3}+c_{4}\right) e_{v_{Y}}-c_{3} \lambda_{2} \chi_{2}+\ddot{Y}_{r e f}\right]
\end{aligned}
$$

where $c_{1}, c_{2}, c_{3}, c_{4}, \lambda_{1}, \lambda_{2}$ are all positive constants.

Table 6 specifies the values for these constants, tuned using the TARA flight simulator with the Design Optimization library.

Following the stability analysis steps as for the altitude control, the stability of 
Table 6: Gains for the integral backstepping position controllers.

\begin{tabular}{|c|c|c|}
\cline { 2 - 3 } \multicolumn{1}{c|}{} & Gain & Value \\
\hline \multirow{4}{*}{ X-axis } & $c_{1}$ & 100.0 \\
\cline { 2 - 3 } & $c_{2}$ & 0.1 \\
\cline { 2 - 3 } & $\lambda_{1}$ & 0.25 \\
\hline \hline \multirow{3}{*}{ Y-axis } & $c_{3}$ & 100.0 \\
\cline { 2 - 3 } & $c_{4}$ & 0.1 \\
\cline { 2 - 3 } & $\lambda_{2}$ & 0.25 \\
\hline \hline \multirow{3}{*}{ Z-axis } & $c_{5}$ & 3.5 \\
\cline { 2 - 3 } & $c_{6}$ & 0.381 \\
\cline { 2 - 3 } & $\lambda_{3}$ & 0.25 \\
\hline
\end{tabular}

lateral control laws is also proved by applying a suitable Lyapunov function $V$,

$$
V=\frac{1}{2}\left[e_{X}^{2}+e_{v_{X_{1}}}^{2}+\lambda_{1} \chi_{1}^{2}\right]
$$

The derivative of $V$ is,

$$
\dot{V}=-c_{1} e_{X}^{2}-c_{2} e_{v_{X_{1}}}^{2} \leq 0
$$

As the $\dot{V}$ is negative semi-definite, the X-axis position control is ensured to be stable. Due to similarity the Y-axis position control stability can also be proved.

\subsection{Fuzzy Control}

In dealing with the uncertainties and disturbances inherent in the urban wind environment, fuzzy logic based controllers appear to offer a promising advantage over some more conventional control techniques. Rather than depending on the formulation of 
a sophisticated mathematical model of system dynamics, fuzzy controllers allow for a model-free design characterized by linguistic descriptions based on knowledge of system behaviour. The fuzzy controller is implemented such that it is independent of plant parameters.

\subsubsection{The Fuzzy Control Law}

Three fuzzy controllers, FLCX, FLCY and FLCZ, are designed to control TARA's position. All three fuzzy controllers are based on a common PD control structure, such that each controller accepts two inputs: the error $(e)$, which is the difference between the desired position and its actual value, and the error rate $(\dot{e})$. The general principles behind fuzzy logic control design dictate the conversion of these crisp analog real-world inputs into fuzzy ones. The fuzzification process assigns a degree of membership between 0 and 1, based on the extent to which the input satisfies the property designated by linguistic terms for corresponding fuzzy sets within the range of all possible input values (commonly referred to as the universe of discourse). For instance, here the fuzzification of the error is achieved by normalizing it to the interval $[-1,+1]$ and assigning grades of membership to the fuzzy sets $\mu_{N}(e), \mu_{Z}(e)$, $\mu_{P}(e)$, labelled with the linguistic terms Negative $(N)$, Zero $(Z)$ and Positive $(P)$. Figure 31 depicts the form these membership functions take for the normalized error input. The $N$ membership function has a trapezoidal shape specified by Equation 89, whereby for an input error value, the degree to which it belongs to the $\mu_{N}(e)$ set is returned. Equations 90 and 91 specify the triangular $Z$ and trapezoidal $P$ membership functions, respectively. 


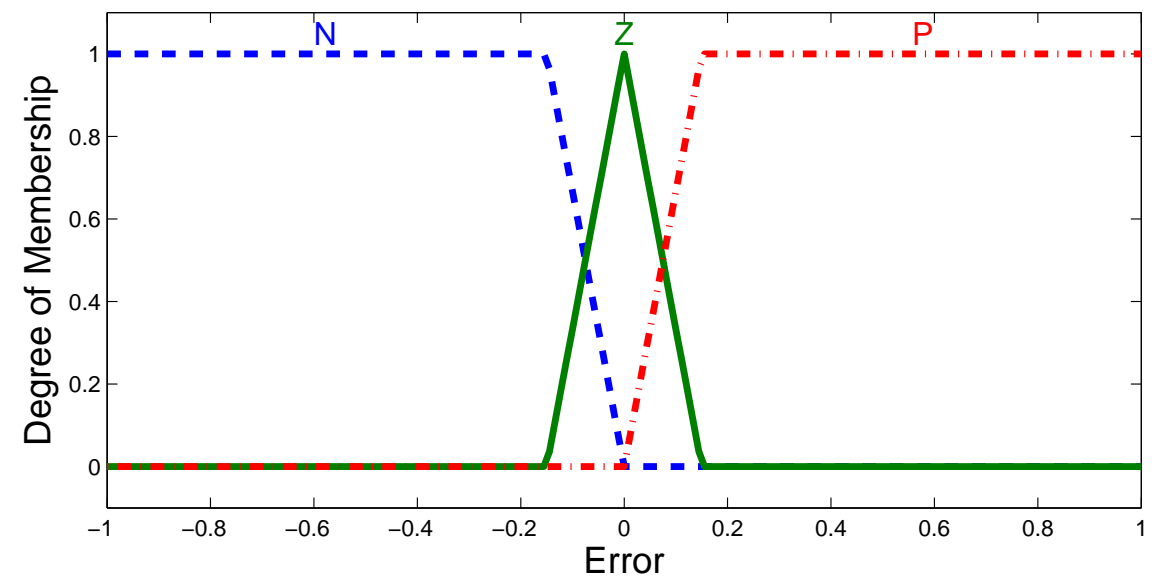

Figure 31: Membership functions for error input.

$$
\begin{gathered}
\mu_{N}(e)= \begin{cases}0, & e>0 \\
\frac{-e}{0.15}, & -0.15 \leq e \leq 0 \\
1, & e<-0.15\end{cases} \\
\mu_{Z}(e)= \begin{cases}\frac{0+0.15}{0.15}, & -0.15<e \leq 0 \\
\frac{0.15-e}{0.15}, & 0<e<0.15 \\
0, & e \geq 0.15\end{cases} \\
\mu_{P}(e)= \begin{cases}\frac{e}{0.15}, & 0 \leq e \leq 0.15 \\
1, & e>0.15\end{cases}
\end{gathered}
$$

Similarly, $N, Z$, and $P$ membership functions are used to fuzzify the input variable error rate, as shown in Figure 32, normalized to the interval $[-3,+3]$, based on the expected $3 \mathrm{~m} / \mathrm{s}$ speed of TARA. The error rate membership functions $\mu_{N}(\dot{e}), \mu_{Z}(\dot{e})$, and $\mu_{P}(\dot{e})$ are given in Equations 92, 93, and 94 . 


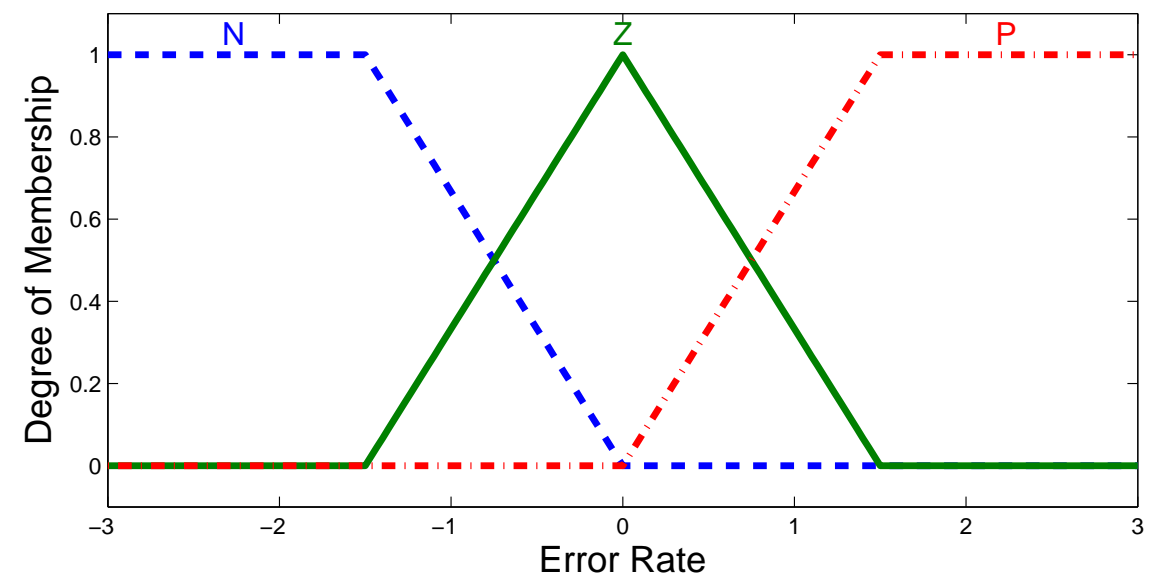

Figure 32: Membership functions for error rate input.

$$
\begin{gathered}
\mu_{N}(\dot{e})= \begin{cases}0, & e>0 \\
\frac{-e}{0.15}, & -0.15 \leq e \leq 0 \\
1, & e<-0.15\end{cases} \\
\mu_{Z}(\dot{e})= \begin{cases}\frac{e+1.5}{1.5}, & -1.5<e \leq 0 \\
\frac{1.5-e}{1.5}, & 0<e<1.5 \\
0, & e \geq 1.5\end{cases} \\
\mu_{P}(\dot{e})= \begin{cases}\frac{e}{0.15}, & 0 \leq e \leq 0.15 \\
1, & e>0.15\end{cases}
\end{gathered}
$$

The output of the fuzzy controller is the control action $U$, which is also fuzzified over a universe of discourse $[-1,1]$, by the three membership functions shown in Figure 33 and listed in Equations 95, 96, and 97. 


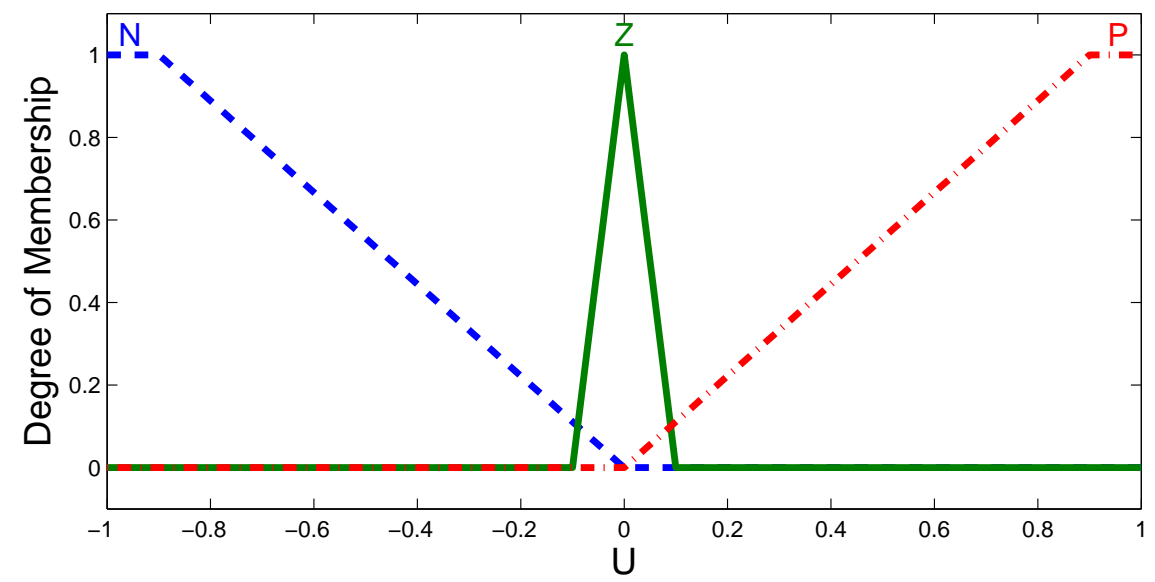

Figure 33: Membership functions for fuzzy output.

$$
\begin{gathered}
\mu_{N}(U)= \begin{cases}0, & e>0 \\
\frac{-e}{0.9}, & -0.9 \leq e \leq 0 \\
1, & e<-0.9\end{cases} \\
\mu_{Z}(U)= \begin{cases}\frac{e+0.1}{0.1}, & -0.1<e \leq 0 \\
\frac{0.1-e}{0.1}, & 0<e<0.1 \\
0, & e \geq 0.1\end{cases} \\
\mu_{P}(U)= \begin{cases}\frac{e}{0.9}, & 0 \leq e \leq 0.9 \\
1, & e>0.9\end{cases}
\end{gathered}
$$

To get from the input to the output membership function, a rule base presented in Table 7 is employed. The fuzzy controller rule base characterizes the control strategy using knowledge of the quadrotor's behaviour based upon experience in flying TARA and controlling its position. These take the form of nine IF-THEN rules, 
Table 7: The fuzzy rule base.

\begin{tabular}{c|c|c|c|c|}
\multicolumn{5}{c}{ Error $(e)$} \\
\cline { 2 - 5 } Error Rate $(\dot{e})$ & $\mathrm{N}$ & $\mathrm{Z}$ & $\mathrm{P}$ \\
\cline { 2 - 5 } & $\mathrm{N}$ & $\mathrm{N}$ & $\mathrm{N}$ & $\mathrm{Z}$ \\
\cline { 2 - 5 } & $\mathrm{Z}$ & $\mathrm{N}$ & $\mathrm{Z}$ & $\mathrm{P}$ \\
\hline $\mathrm{P}$ & $\mathrm{Z}$ & $\mathrm{P}$ & $\mathrm{P}$ \\
\hline
\end{tabular}

called implications, such as IF $e$ is $N$ AND $\dot{e}$ is $N$ THEN output $(U)$ is $N$, as shown in Table 7 . The resultant from the implication is then used to produce the fuzzy output, $U$. For example, consider an error value of $e=0.036$ and error rate $\dot{e}=-0.622$. Evaluating the degree of membership values for the specified error, gives us $\mu_{N}(e=0.036)=0, \mu_{Z}(e=0.036)=0.76$, and $\mu_{P}(e=0.036)=0.24$. Similarly, for the given error rate, $\mu_{N}(\dot{e}=-0.622)=0.41, \mu_{Z}(\dot{e}=-0.622)=0.59$, and $\mu_{P}(\dot{e}=-0.622)=0$, as presented in Figure 34. A min operation, shown in Equation 98, performs the fuzzy AND operation, determining which of the nine rules will fire, based on having a min membership greater than zero. Hence, for the given inputs, the rule IF $e$ is $N$ AND $\dot{e}$ is $N$ THEN output $(U)$ is $N$ will not fire, as $\min \left[\mu_{N}(e=0.036), \mu_{N}(\dot{e}=-0.622)\right]=\min [0,0.41]=0$. The Mamdani implication method given in Equation 99 is employed here, whereby the min value is used as the firing strength of the rule under consideration. These firing strengths determine the degree of membership of the output membership functions for each selected rule, as shown in Figure 34. Thus, the rule IF $e$ is $Z$ AND $\dot{e}$ is $N$ THEN output $(U)$ is $N$ has a firing strength of $\min \left[\mu_{Z}(e=0.036), \mu_{N}(\dot{e}=-0.622)\right]=\min [0.76,0.41]=0.41$. The maximum value of membership, per Equation 100, in each of the N, Z, and P fuzzy sets is then used as a cutoff value for aggregating each set. The three resulting sets are then unified to obtain a single output membership function, as depicted in Figure 35 in the bottom graph. This fuzzy output is converted into a crisp output 
$U_{\text {crisp }}=-0.129$ by calculating the centroid of area (Equation 101) of the fuzzy output function. The control surface plot shown in Figure 36 presents a graphical depiction of how the resulting output varies as a function of the error and error rate signals.

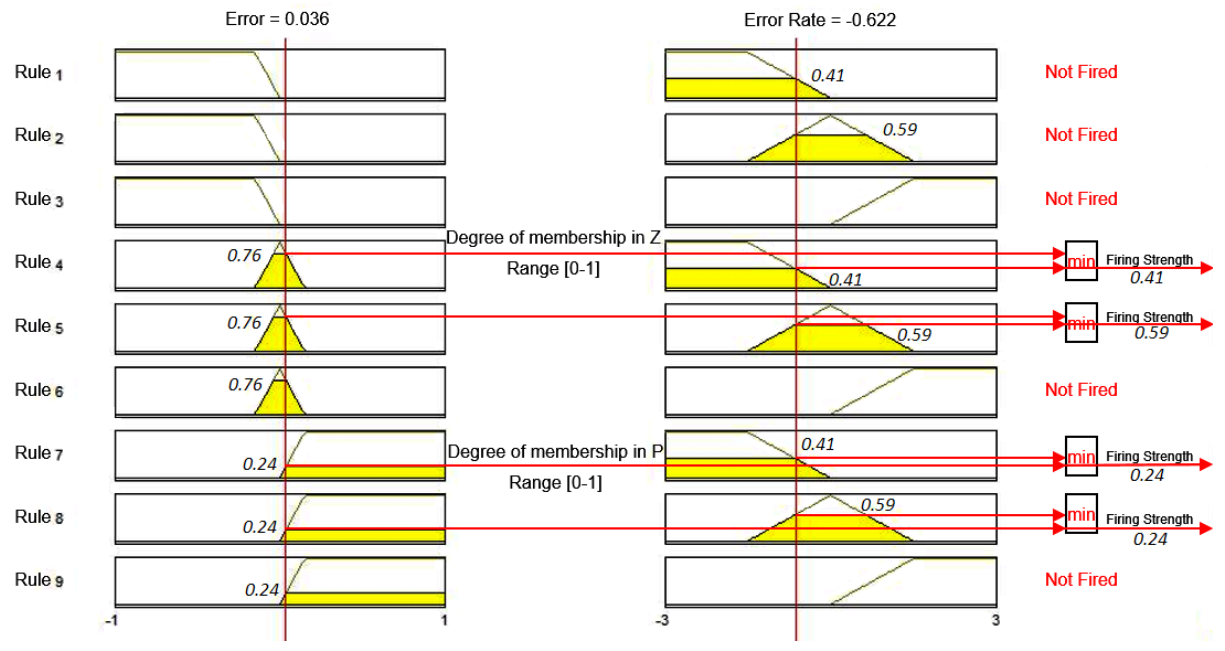

Figure 34: Mamdani fuzzy inferencing method.

$$
\begin{gathered}
\mu_{A \cap B}(x)=\min \left[\mu_{A}(x), \mu_{B}(x)\right] \quad \forall x \in X \\
\mu_{A \rightarrow B}(x, y)=\min \left[\mu_{A}(x), \mu_{B}(y)\right] \quad \forall x \in X, \forall y \in Y \\
\mu_{A \cup B}(x)=\max \left[\mu_{A}(x), \mu_{B}(x)\right] \quad \forall x \in X \\
U_{\text {crisp }}=Z_{C O A}=\frac{\int_{Z} \mu_{C}(z) z d z}{\int_{Z} \mu_{C}(z) d z}
\end{gathered}
$$

In order for it to be modular and independent of TARA's parameters, the fuzzy logic controllers are bounded by pre-processing and post-processing blocks elaborated in Section 3.1.4 of Chapter 3. The pre-processing module calculates error $e$ of the 


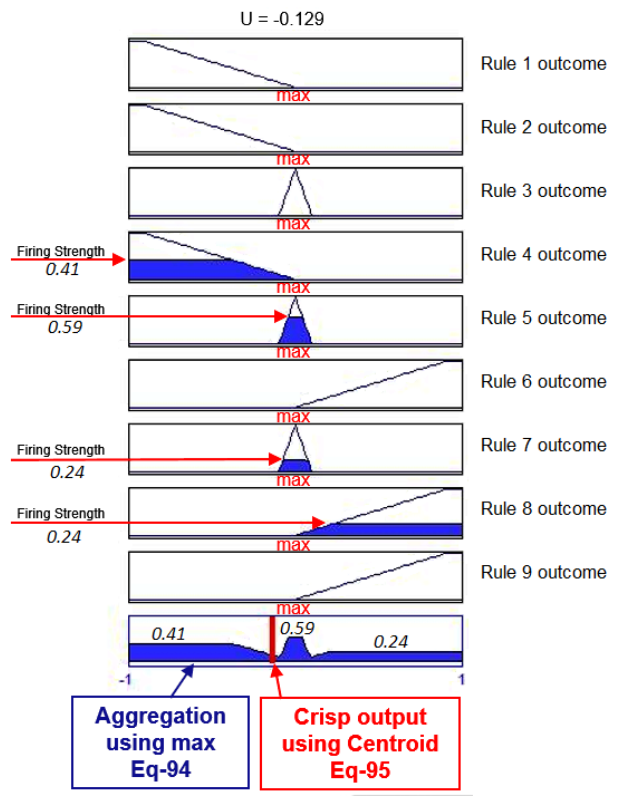

Figure 35: Aggregation method and crisp output.

specified states of the quadrotor which need to be controlled. Error rate $\dot{e}$ is also evaluated and both $e$ and $\dot{e}$ are scaled and normalized according to the input requirements of the fuzzy controller. The post-processing block, Figure 6, uses the controller's output signals to calculate the pulse width modulation (PWM) value of each motor. The resultant PWM values are saturated to a threshold range of 250 to 500 to match the input requirements of the motors used. Here again it is of note that this control

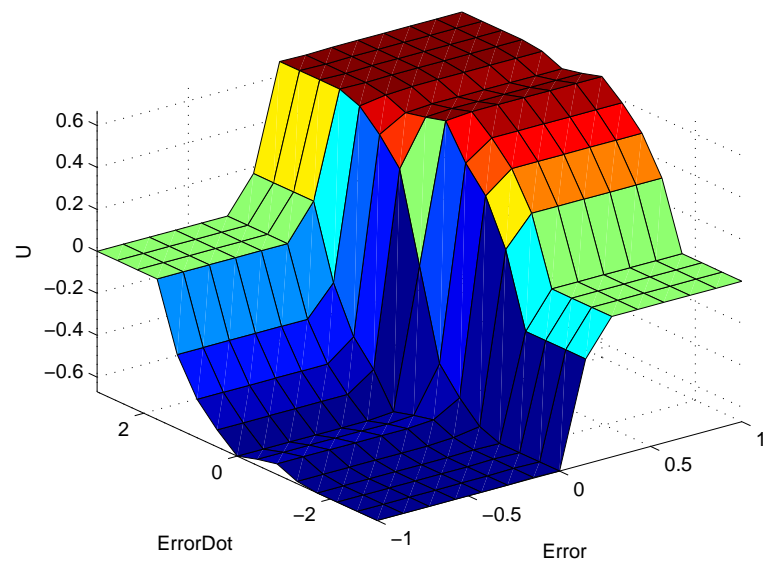

Figure 36: The surface plot of the fuzzy position controller. 
scheme does not depend on kinematic and dynamic equations. Therefore, changing the quadrotor or some of its physical parameters like the mass or inertia does not require altering the fuzzy logic controller. Instead, the post-processing module may need to be fine-tuned to optimize the controller's performance.

The implementation of a fuzzy controller necessitates a high computational capability from the onboard processors. The onboard computation on TARA is carried out by an Arduino board containing an 8-bit processor running at $16 \mathrm{MHz}$, which is not capable of running a full fuzzy inference system, let alone three fuzzy controllers. Therefore, an alternative of translating the fuzzy controller into a reasonable sized lookup table is implemented. The lookup table is a $15 \times 15$ value $2 \mathrm{D}$ array which approximates the control surface shown in Figure 36. Intermediate values are calculated by interpolation.

\subsubsection{Fuzzy Altitude Control}

To control the altitude of TARA we start by defining the Z-axis error as the difference between the desired reference altitude and the actual current altitude:

$$
e_{Z}=Z_{r e f}-Z
$$

The error rate is:

$$
\dot{e}_{Z}=\frac{d}{d t}\left(e_{Z}\right)
$$

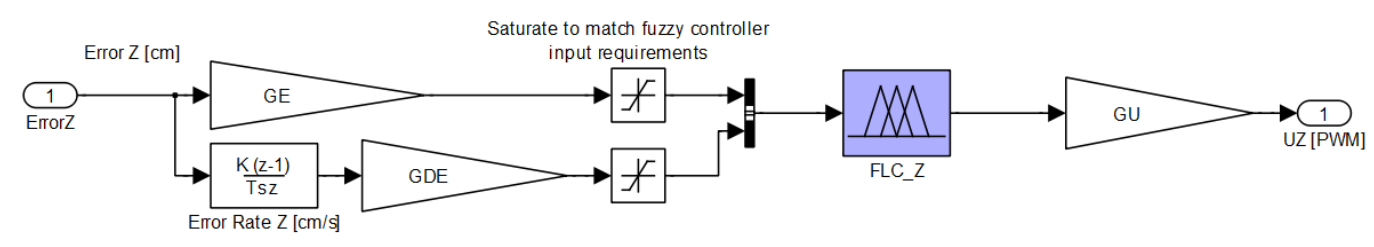

Figure 37: The fuzzy altitude controller.

As shown in the Figure 37, the error and error rate values are normalized to the 
respective intervals $[-1,+1]$ and $[-3,+3]$ to match the input requirements of the fuzzy controller, using the gains $G E=2.86 \times 10^{-4}$ and $G D E=0.01$, respectively. The output of the fuzzy controller, the control action $\mathrm{U}$ is also scaled by a gain $G U=40$. The resulting output is post-processed as shown in Figure 7 to determine the PWM value for each motor in order to produce the required thrust to achieve the desired altitude.

\subsubsection{Fuzzy Lateral Position Control}

The lateral position of TARA is controlled by two fuzzy controllers, one for the $\mathrm{X}$ and one for the $\mathrm{Y}$ axis. The errors on the $\mathrm{X}$ and $\mathrm{Y}$ axes are defined as:

$$
\begin{gathered}
e_{X}=X_{r e f}-X \\
e_{Y}=Y_{r e f}-Y
\end{gathered}
$$

Similarly, the error rates for $\mathrm{X}$ and $\mathrm{Y}$ axes are defined as:

$$
\begin{aligned}
& \dot{e}_{X}=\frac{d}{d t}\left(e_{X}\right) \\
& \dot{e}_{Y}=\frac{d}{d t}\left(e_{Y}\right)
\end{aligned}
$$

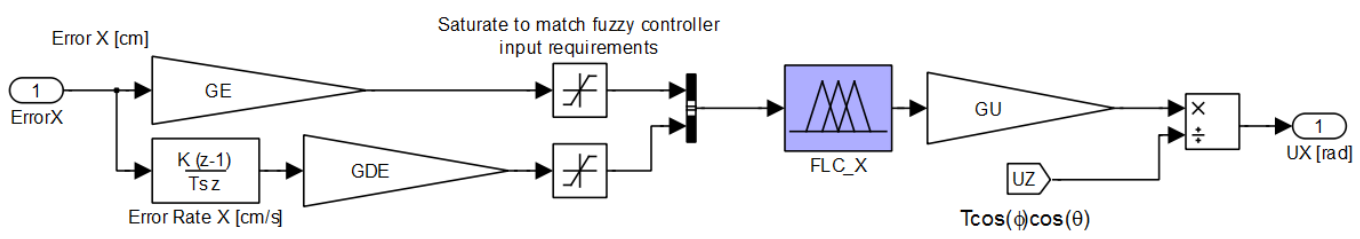

Figure 38: The fuzzy $\mathrm{X}$-axis position controller.

Due to symmetry a common scheme of gains is used for the $\mathrm{X}$ and $\mathrm{Y}$ axis control. 
The $\mathrm{X}$-axis position control is shown as an example in Figure 38, where the error and error rate inputs, as well as the control action output $U$ are scaled using gains $G E=2.94 \times 10^{-5}, G D E=0.0036$, and $G U=300$ respectively. Here, the resulting output is divided by the Z-axis component of cumulative thrust command $T$ sent to the motors. This is done in order to minimize the impact of altitude controller effort on the lateral control. The final output of the $\mathrm{X}$ and $\mathrm{Y}$ position controllers are the desired pitch and roll angles in radians, respectively, for the attitude controller shown in Figure 6. The output of the attitude controllers and the altitude control is post-processed as shown in Figure 7 to produce appropriate PWM command for each motor.

\subsection{Adaptive Control}

Adaptive control seeks to change a given control law in the presence of uncertainties without prior knowledge about the bounds on these uncertainties. Section 4.2 presented an integral backstepping controller which employed static feedback and guaranteed the boundedness of its closed-loop state in the presence of uncertain bounded nonlinearities. Modifying the integral backstepping control by applying a dynamic nonlinear integral feedback based on an estimate of the disturbance, an adaptive integral backstepping (AIB) controller can be designed. A parameter update law is used to continuously update the feedback of the AIB controller making the control dynamic and providing adaptation to new parameter estimates. While the resulting AIB controller is complex in its design when compared with its non-adaptive integral backstepping counterpart, in return it not only guarantees state boundedness, but also provides the sought after system regulation by converging the controlled states to their desired reference values in the presence of perturbations and disturbances. 


\subsubsection{Adaptive Integral Backstepping Altitude Control}

Consider the simplified equation of motion for the Z-axis from Equation 48,

$$
\ddot{Z}=g-\frac{1}{M} T \cos (\theta) \cos (\phi)+D_{Z}
$$

where $Z$ is the quadrotor's altitude, $\ddot{Z}$ is the acceleration along the z-axis, $g$ is the acceleration due to gravity, $M$ is the total mass of TARA, $T$ is the total thrust, and $D_{Z}$ represents an uncertainty in the form of a constant but unknown disturbance.

Following the conventional backstepping design procedure, we define the difference between the desired Z-axis position, $Z_{\text {ref }}$, and the actual Z-axis position, $Z$, as the altitude tracking error,

$$
e_{Z}=Z_{r e f}-Z
$$

along with the rate at which the tracking error changes with time,

$$
\dot{e}_{Z}=\frac{d e_{Z}}{d t}=\dot{Z}_{r e f}-\dot{Z}
$$

Here, $\dot{Z}$ is a system state variable which we cannot control directly. Instead, we can specify a desired behavior for $\dot{Z}$ as a virtual control, $v_{Z_{1}}$, such that $\dot{Z}$ tries to follow $v_{Z_{1}}$, which is composed of terms which are proportional to the tracking error, $e_{Z}$, its integral, and a term which accounts for a change in the desired altitude,

$$
v_{Z_{1}}=c_{5} e_{Z}+\dot{Z}_{r e f}+\lambda_{3} \chi_{3}
$$

where $c_{5}$ and $\lambda_{3}$ are positive constants, and $\chi_{3}=\int e_{Z}(\Delta t) \partial \Delta t$.

The effectiveness of this virtual control can be measured by its own tracking error, $e_{v_{Z_{1}}}$,

$$
e_{v_{1}}=v_{Z_{1}}-\dot{Z}=c_{5} e_{Z}+\dot{Z}_{r e f}+\lambda_{3} \chi_{3}-\dot{Z}
$$


The derivative of this tracking error becomes,

$$
\dot{e}_{v_{Z_{1}}}=\frac{e_{v_{Z_{1}}}}{d t}=c_{5} \dot{e}_{Z}+\ddot{Z}_{r e f}+\lambda_{3} e_{Z}-\ddot{Z}
$$

Isolating for $\dot{Z}_{\text {ref }}-\dot{Z}$ in Equation 112 and using this result in Equation 110 yields,

$$
\dot{e}_{Z}=\frac{d e_{Z}}{d t}=e_{v_{Z_{1}}}-c_{5} e_{Z}-\lambda_{3} \chi_{3}
$$

Substituting Equation 114 into Equation 113, and replacing the acceleration in the $\mathrm{Z}$ direction, $\ddot{Z}$, using the quadrotor dynamics as represented by Equation 108, we obtain the rate of change of error of the virtual control (which is identical to Equation 57 but with an addition of the uncertain disturbance term),

$$
\dot{e}_{v_{1}}=\frac{d e_{v_{Z_{1}}}}{d t}=c_{5}\left(e_{v_{Z_{1}}}-c_{5} e_{Z}-\lambda_{3} \chi_{3}\right)+\ddot{Z}_{r e f}+\lambda_{3} e_{Z}-\left(g-\frac{U_{Z} \cos (\theta) \cos (\phi)}{M}\right)-D_{Z}
$$

Another virtual controller which depends on $e_{Z}$ and $e_{v_{Z_{1}}}, v_{Z_{2}}$, can be introduced as a desired behaviour for the rate of change of $e_{v_{Z_{1}}}$,

$$
\dot{e}_{{Z_{1}}_{1}}=v_{Z_{2}}=-c_{6} e_{{Z_{1}}_{1}}-e_{Z}
$$

From the expression for $\dot{e}_{v_{Z_{1}}}$ in Equation 115 we can write,

$$
-c_{6} e_{v_{1}}-e_{Z}=c_{5}\left(e_{v_{Z_{1}}}-c_{5} e_{Z}-\lambda_{3} \chi_{3}\right)+\ddot{Z}_{r e f}+\lambda_{3} e_{Z}-\left(g-\frac{U_{Z} \cos (\theta) \cos (\phi)}{M}+D_{Z}\right)
$$

Solving for the control function $U_{Z}$ yields,

$$
U_{Z}=\frac{M}{\cos (\theta) \cos (\phi)}\left[g+\left(c_{5}+c_{6}\right) e_{v_{1}}+\left(1-c_{5}^{2}+\lambda_{3}\right) e_{Z}-c_{5} \lambda_{3} \chi_{3}+\ddot{Z}_{r e f}-D_{Z}\right]
$$

Here we do not know the actual disturbance $D_{Z}$ in the system; therefore, we will 
replace $D_{Z}$ with an estimated value, $\hat{D}_{Z}$,

$$
U_{Z}=\frac{M}{\cos (\theta) \cos (\phi)}\left[g+\left(c_{5}+c_{6}\right) e_{v_{Z_{1}}}+\left(1-c_{5}^{2}+\lambda_{3}\right) e_{Z}-c_{5} \lambda_{3} \chi_{3}+\ddot{Z}_{r e f}-\hat{D}_{Z}\right]
$$

An adaptation law needs to be derived in order to update the estimated $\hat{D}_{Z}$ in our control law. Let the disturbance estimate error $\tilde{D}_{Z}$ be,

$$
\tilde{D}_{Z}=\hat{D}_{Z}-D_{Z}
$$

The estimated parameter $\hat{D}_{Z}$ is modeled such that its derivative is negative definite,

$$
\frac{d \hat{D}_{Z}}{d t}=-\gamma_{Z} e_{v_{Z_{1}}}
$$

and

$$
\hat{D}_{Z}=-\gamma_{Z} \chi_{Z}
$$

where $\chi_{Z}=\int e_{v_{Z_{1}}}(\Delta t) \partial \Delta t$ and the adaptation gain $\gamma_{Z}$ is a positive value.

The final control input containing the adaptation law is derived as,

$$
U_{Z}=\frac{M}{\cos (\theta) \cos (\phi)}\left[g+\left(c_{5}+c_{6}\right) e_{{Z_{1}}_{1}}+\left(1-c_{5}^{2}+\lambda_{3}\right) e_{Z}-c_{5} \lambda_{3} \chi_{3}+\ddot{Z}_{r e f}+\gamma_{Z} \chi_{Z}\right]
$$

Table 8 lists the values for the gains for the positive constants $c_{5}, c_{6}, \lambda_{3}$, a, and $\gamma_{Z}$. With the exception of the adaptation gain, $\gamma_{Z}$, which is tuned manually in the simulator the remaining parameters are the same as those used in the integral backstepping control. 


\subsubsection{Stability Proof of AIB Altitude Control Law}

A candidate Lyapunov function may be selected as,

$$
V=\frac{1}{2} e_{Z}^{2}+\frac{1}{2} e_{v_{Z_{1}}}^{2}+\frac{\lambda_{3}}{2} \chi_{3}^{2}+\frac{1}{2 \gamma_{Z}} \tilde{D}_{Z}^{2}
$$

The derivative of the Lyapunov function is:

$$
\dot{V}=e_{Z} \dot{e}_{Z}+e_{v_{Z_{1}}} \dot{e}_{v_{Z_{1}}}+\lambda_{3} \chi_{3} \dot{\chi}_{3}+\frac{\tilde{D}_{Z}}{\gamma_{Z}} \frac{d \hat{D}_{Z}}{d t}
$$

Noting that by substituting $U_{Z}$ from Equation 119 in Equation 115 we get,

$$
\dot{e}_{v_{Z_{1}}}=\frac{d e_{v_{Z_{1}}}}{d t}=-c_{6} e_{v_{Z_{1}}}-e_{Z}+\tilde{D}_{Z}
$$

the derivative of the Lyapunov function becomes,

$$
\begin{gathered}
\dot{V}=e_{Z}\left(e_{v_{Z_{1}}}-c_{5} e_{Z}-\lambda_{3} \chi_{3}\right)+e_{v_{Z_{1}}}\left(-c_{6} e_{v_{Z_{1}}}-e_{Z}+\tilde{D}_{Z}\right)+\lambda_{3} \chi_{3} e_{Z}+\frac{\tilde{D}_{Z}}{\gamma_{Z}} \frac{d \hat{D}_{Z}}{d t} \\
\dot{V}=e_{Z} e_{v_{Z_{1}}}-c_{5} e_{Z}^{2}-\lambda_{3} \chi_{3} e_{Z}-c_{6} e_{v_{Z_{1}}}^{2}-e_{Z} e_{v_{Z_{1}}}+e_{v_{Z_{1}}} \tilde{D}_{Z}+\lambda_{3} \chi_{3} e_{Z}+\frac{\tilde{D}_{Z}}{\gamma_{Z}} \frac{d \hat{D}_{Z}}{d t} \\
\dot{V}=-c_{5} e_{Z}^{2}-c_{6} e_{v_{Z_{1}}}^{2}+\tilde{D}_{Z}\left(e_{v_{Z_{1}}}+\frac{1}{\gamma_{Z}} \frac{d \hat{D}_{Z}}{d t}\right) \\
\dot{V}=-c_{5} e_{Z}^{2}-c_{6} e_{v_{Z_{1}}}^{2}+\tilde{D}_{Z}\left(e_{v_{Z_{1}}}+\frac{-\gamma_{Z} e_{v_{Z_{1}}}}{\gamma_{Z}}\right)
\end{gathered}
$$




$$
\dot{V}=-c_{5} e_{Z}^{2}-c_{6} e_{v_{Z_{1}}}^{2} \leq 0
$$

Given that the derivative $\dot{V}$ is negative semi-definite and $\dot{V}(0)=0$, the stability of the system is guaranteed.

\subsubsection{Adaptive Integral Backstepping Lateral Position Con- trol}

Similarly, AIB control laws are derived for the $\mathrm{X}$ and $\mathrm{Y}$ axes. The simplified system equations for $\mathrm{X}$-axis and $\mathrm{Y}$-axis motion are

$$
\begin{gathered}
\ddot{X}=\frac{\left(U_{X}\right)}{M} T+D_{X} \\
\ddot{Y}=\frac{\left(-U_{Y}\right)}{M} T+D_{Y}
\end{gathered}
$$

Following the same derivation as that of the altitude control law we can write the $\mathrm{X}$ and $\mathrm{Y}$-axis AIB control laws as:

$$
\begin{aligned}
& U_{X}=\frac{M}{T}\left[\left(1-c_{1}^{2}+\lambda_{1}\right) e_{X}+\left(c_{1}+c_{2}\right) e_{v_{X_{1}}}-c_{1} \lambda_{1} \chi_{1}+\ddot{X}_{r e f}+\gamma_{X} \chi_{X}\right] \\
& U_{Y}=-\frac{M}{T}\left[\left(1-c_{3}^{2}+\lambda_{2}\right) e_{Y}+\left(c_{3}+c_{4}\right) e_{V_{Y_{1}}}-c_{3} \lambda_{2} \chi_{2}+\ddot{Y}_{r e f}+\gamma_{Y} \chi_{Y}\right]
\end{aligned}
$$

where $c_{1}, c_{2}, c_{3}, c_{4}, \lambda_{1}, \lambda_{2}, \gamma_{X}$, and $\gamma_{Y}$ are all positive constants, whose values are presented in Table 8. 
Table 8: Gains for the adaptive integral backstepping position controllers.

\begin{tabular}{|c|c|c|}
\cline { 2 - 3 } \multicolumn{1}{c|}{} & Gain & Value \\
\hline \multirow{4}{*}{ X-axis } & $c_{1}$ & 100.0 \\
\cline { 2 - 3 } & $c_{2}$ & 0.1 \\
\cline { 2 - 3 } & $\lambda_{1}$ & 0.25 \\
\cline { 2 - 3 } & $\gamma_{X}$ & 0.0001 \\
\hline \hline \multirow{5}{*}{ Y-axis } & $c_{3}$ & 100.0 \\
\cline { 2 - 3 } & $c_{4}$ & 0.1 \\
\cline { 2 - 3 } & $\lambda_{2}$ & 0.25 \\
\cline { 2 - 3 } & $\gamma_{Y}$ & 0.0001 \\
\hline \hline \multirow{5}{*}{ Z-axis } & $c_{5}$ & 3.5 \\
\cline { 2 - 3 } & $c_{6}$ & 0.381 \\
\cline { 2 - 3 } & $\lambda_{3}$ & 0.25 \\
\cline { 2 - 3 } & $\gamma_{Z}$ & 0.00035 \\
\hline
\end{tabular}

\subsection{Step Response Performance Evaluation}

Having established four different controllers based on varying methodologies, their performance using the constants listed in Tables 5, 6, and 8, is tested in response to a $10 \mathrm{~m}$ step input on each of the X, Y, and Z-axes. The simulation results are analyzed in terms of standard step response metrics, namely the undershoot, overshoot, rise time and settling time. Here, the rise time is taken as the time the response requires to rise from $10 \%$ to $90 \%$ of the reference value. The settling time is defined as the time needed for the control response to reach, and stay within $10 \%$ of the desired setpoint. 


\subsubsection{X-axis Step Response}

The $\mathrm{X}$-axis position control and corresponding pitch angle in response to a $10 \mathrm{~m}$ step input for the PD, IB, fuzzy, and AIB controllers is depicted in Figure 39. The step response characteristics of the $\mathrm{X}$-axis motion are compiled in Table 9 . On the whole, the results provided by the four control methods are of a similar overdamped nature, with all of the controllers demonstrating the stable, restrained motion intended for the TARA quadrotor. However, the slight differences between the response characteristics obtained highlight the tradeoff which exists between faster responses and better stability. The IB and AIB controllers provide the quickest rise times, with identical values of $14.7 \mathrm{sec}$, compared with the 19.6 and $20.0 \mathrm{sec}$ required by the PD and fuzzy controllers, respectively. Similarly, the AIB controller has a settling time of $17.2 \mathrm{sec}$, and the IB controller of $17.3 \mathrm{sec}$, while the PD control's settling time is $21.7 \mathrm{sec}$, and the fuzzy control's is $23.6 \mathrm{sec}$. On the other hand, the PD and fuzzy controls display better stability once they reach the setpoint, as well as lower overshoots of $1.2 \%$ and $1.1 \%$, respectively. Still, the $4.2 \%$ and $4.7 \%$ overshoots, as well as the $1.8 \%$ and $2.0 \%$ undershoots evidenced by the IB and AIB controls respectively are quite small as compared with typical overshoot and undershoot profiles.

As desired, the corresponding pitch angles all maintain a level response, with small attitude adjustments in evidence. The IB, fuzzy, and AIB controller pitch angles remain within the "good" range of \pm 5 degrees, as specified by the attitude stabilization metric defined in Table 4. The PD controller shows the greatest angular variation, rapidly deviating from -5.9 to $4.1 \mathrm{deg}$ in response to the step reference. The fuzzy pitch angle has the least variability, with a small but sustained effort in reaction to the step input. 


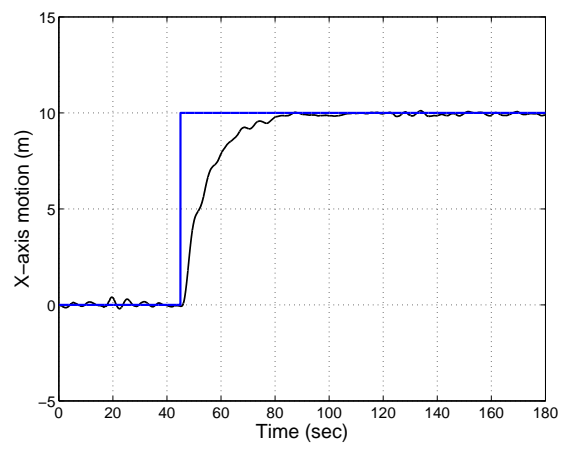

(a) PD

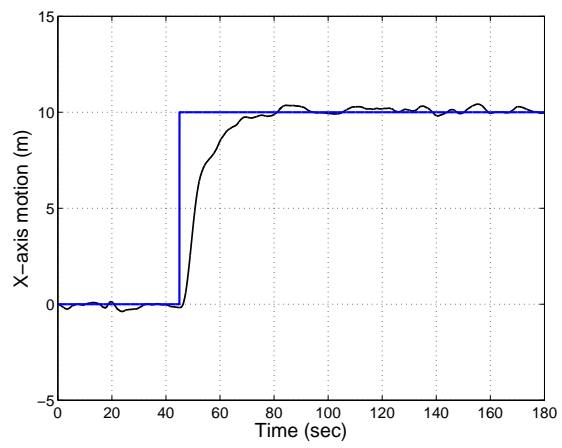

(c) IB

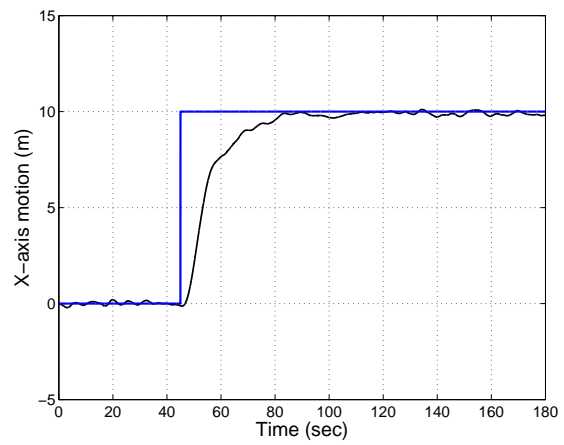

(e) Fuzzy

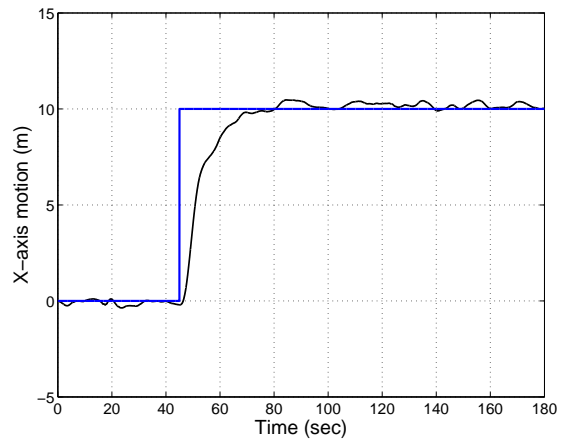

(g) AIB

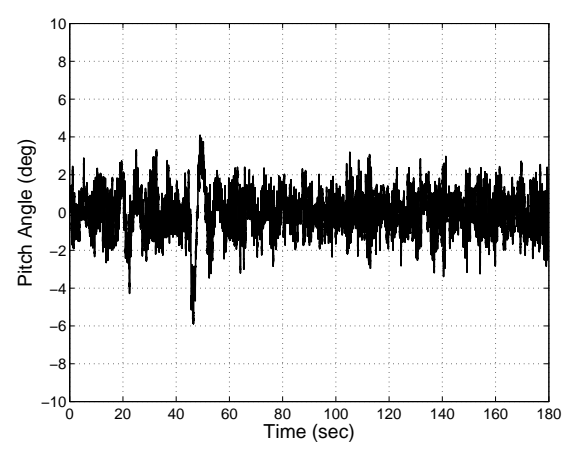

(b) Pitch angle for PD

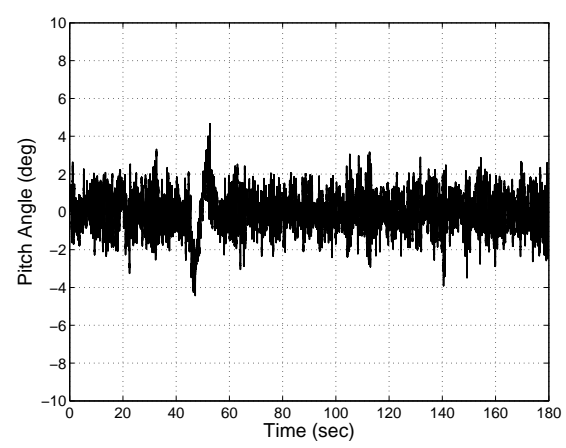

(d) Pitch angle for IB

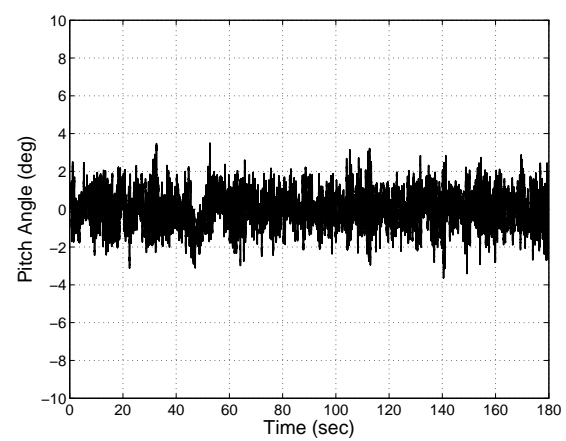

(f) Pitch angle for fuzzy

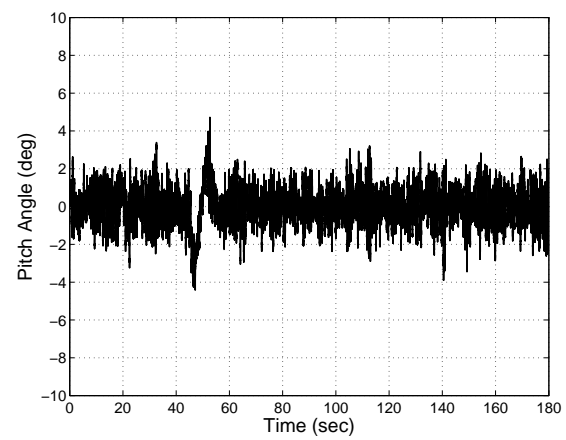

(h) Pitch angle for AIB

Figure 39: $\mathrm{X}$-axis step response. 
Table 9: X-axis step response summary.

\begin{tabular}{|l|c|c|c|c|}
\cline { 2 - 5 } \multicolumn{1}{c|}{} & PD & IB & Fuzzy & AIB \\
\hline Undershoot (\%) & 0.7 & 1.8 & 1.3 & 2.0 \\
\hline Overshoot (\%) & 1.2 & 4.2 & 1.1 & 4.7 \\
\hline Rise Time (sec) & 19.6 & 14.7 & 20.0 & 14.7 \\
\hline Settling Time (sec) & 21.7 & 17.3 & 23.6 & 17.2 \\
\hline
\end{tabular}

\subsubsection{Y-axis Step Response}

The Y-axis step response performance for a $10 \mathrm{~m}$ step input is presented in Figure 40. As indicated in Table 10, minimal $0.2 \%$ undershoots occur for the PD, IB and AIB responses, with the fuzzy response devoid of an undershoot altogether. As was the case for the $\mathrm{X}$-axis, the IB and AIB controllers present the fastest rise times, as well as settling times. The fuzzy controller has an extended settling time of $30.4 \mathrm{sec}$, and requires $26.8 \mathrm{sec}$ of rise time. Again, however, the $4.3 \%$ and $5.6 \%$ overshoots presented by the IB and AIB controllers, respectively, are higher than the $0.6 \%$ overshoot for the fuzzy controller, or the $0.7 \%$ overshoot for the PD controller.

Considering the associated roll angles for each of the controllers, the PD response shows the greatest fluctuation in attitude, and takes the longest time to stabilize following the step input. The fuzzy controller places the least demand on the roll angle, maximally varying between -3.3 and 3.9 degrees. The IB and AIB pitch responses navigate a middle ground between the large fast deflections of the PD roll angle and the limited but sustained response of the fuzzy controller.

\subsubsection{Z-axis Step Response}

Table 11 summarizes the Z-axis step input response for the PID, IB, fuzzy, and AIB controllers. As illustrated in Figure 41, the PID, IB and AIB control efforts 


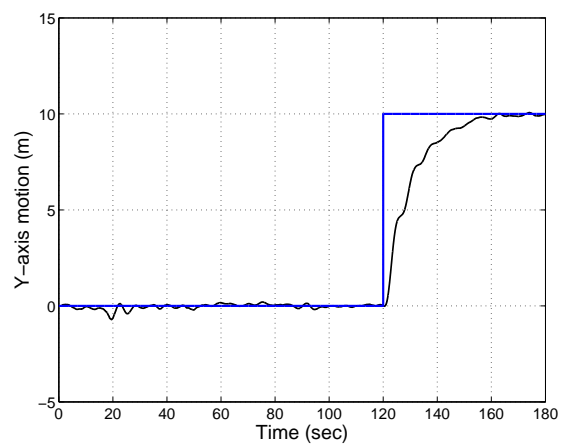

(a) PD

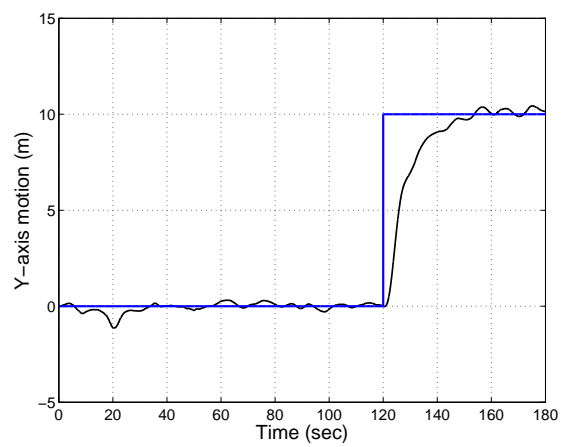

(c) IB

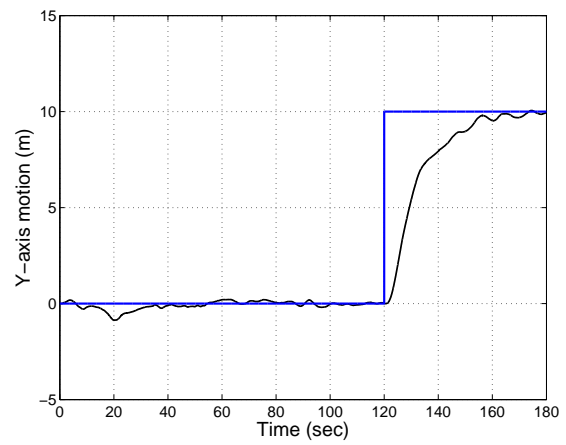

(e) Fuzzy

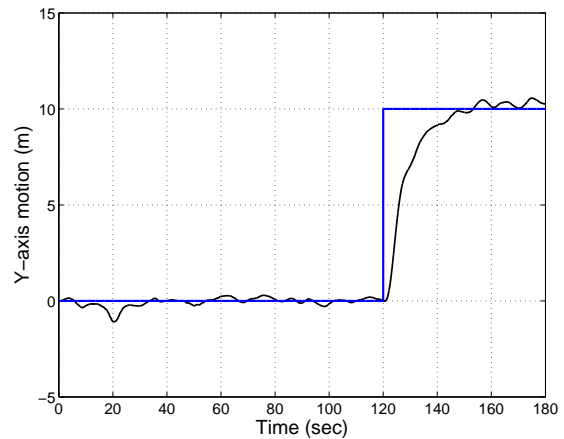

(g) AIB

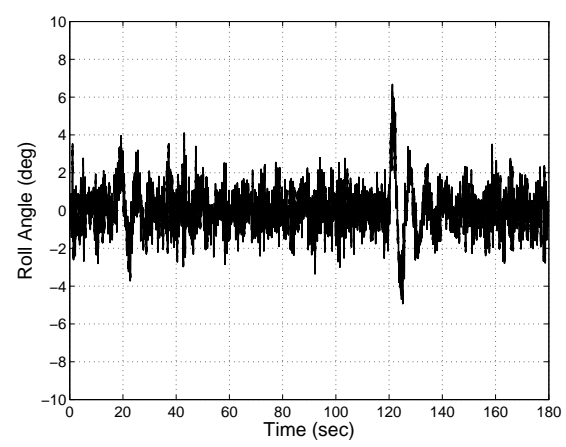

(b) Roll angle for PD

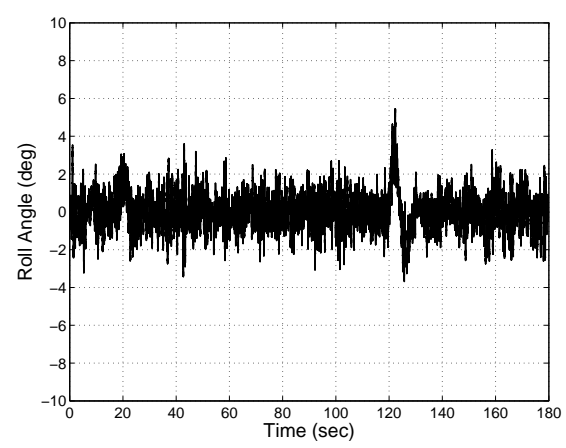

(d) Roll angle for IB

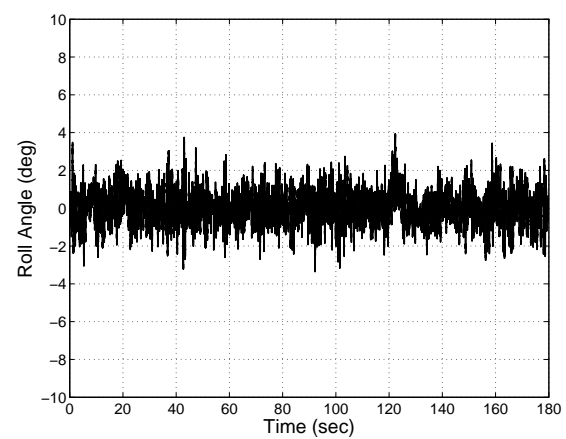

(f) Roll angle for fuzzy

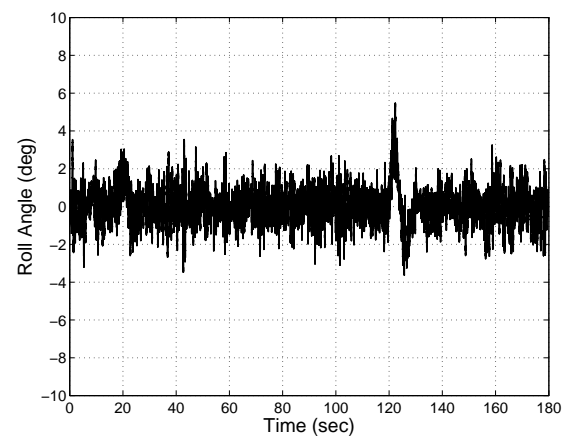

(h) Roll angle for AIB

Figure 40: Y-axis step response. 
Table 10: Y-axis step response summary.

\begin{tabular}{|l|c|c|c|c|}
\cline { 2 - 5 } \multicolumn{1}{c|}{} & PD & IB & Fuzzy & AIB \\
\hline Undershoot (\%) & 0.2 & 0.2 & 0.0 & 0.2 \\
\hline Overshoot (\%) & 0.7 & 4.3 & 0.6 & 5.6 \\
\hline Rise Time (sec) & 21.7 & 16.2 & 26.8 & 15.2 \\
\hline Settling Time (sec) & 23.8 & 18.8 & 30.4 & 17.9 \\
\hline
\end{tabular}

depict a classical underdamped response. In comparison with the $\mathrm{X}$-axis and $\mathrm{Y}$-axis simulations, much faster rise and settling times are observed, with the IB and AIB controllers demonstrating equal $2.7 \mathrm{sec}$ rise times, followed closely by the $2.9 \mathrm{sec}$ rise time of the PID controller. The nature of Z-axis motion is different from that of the $\mathrm{X}$ and $\mathrm{Y}$-axes, purely due to the presence of gravitational force. Furthermore, any substantial Z-axis motion produces a big influence on the $\mathrm{X}$-axis and $\mathrm{Y}$-axis position control. Therefore, it is desirable to have a faster altitude control, to quickly stabilize and damp the altitude before lateral position control can be achieved. For the PID, IB, and AIB controllers, considerable overshoots are observed, with values of $27.1 \%$, $28.5 \%$, and $28.7 \%$, respectively. For the Z-axis case the fuzzy controller distinguishes itself from the other applied techniques, with its critically damped response producing an overshoot of only $1.3 \%$ and the fastest settling time of 7.2 sec, albeit with a somewhat slower rise time of $5.7 \mathrm{sec}$. The PID response is the most sluggish in achieving and maintaining the desired setpoint, needing $18.1 \mathrm{sec}$ of settling time.

The yaw angle is well stabilized by all four controllers and is consistently retained well within the bounds of a "good" performance ( \pm 5 degrees). A main factor contributing to the good yaw stabilization performance in simulation is that all four motors are modelled as identical, and compass bias due to EMI effects are not modelled. 


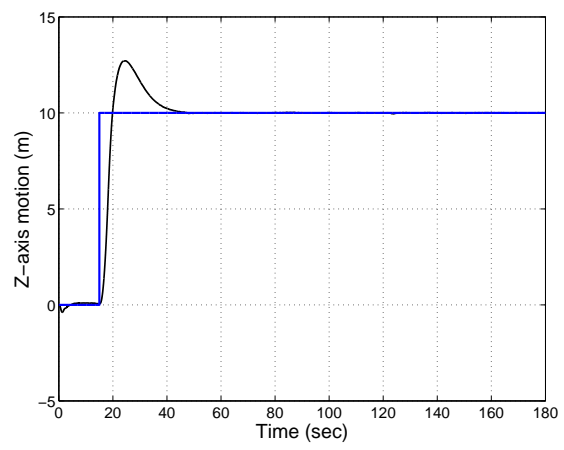

(a) PID

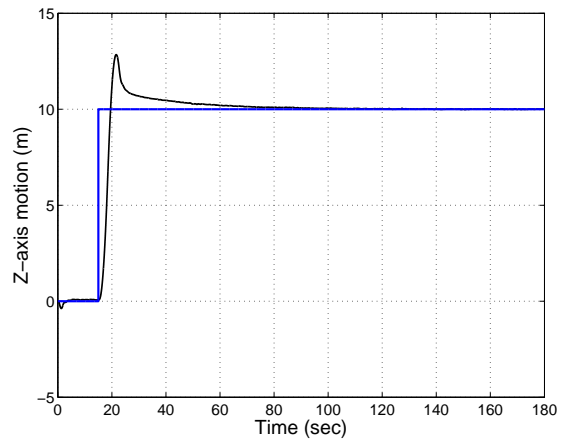

(c) IB

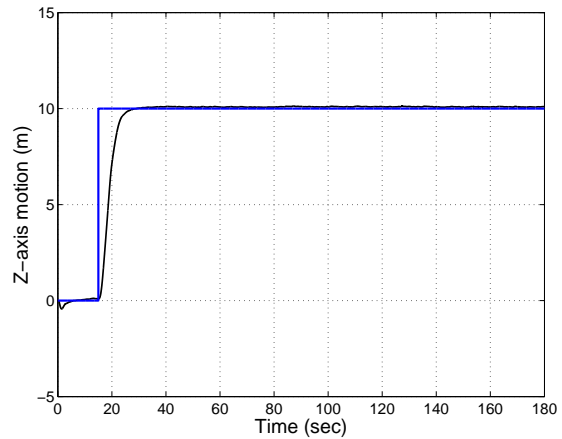

(e) Fuzzy

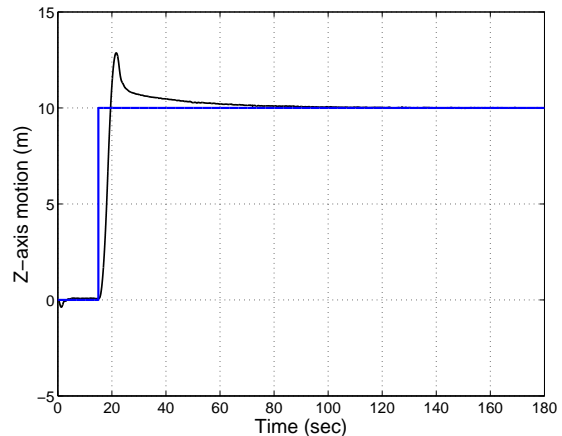

(g) AIB

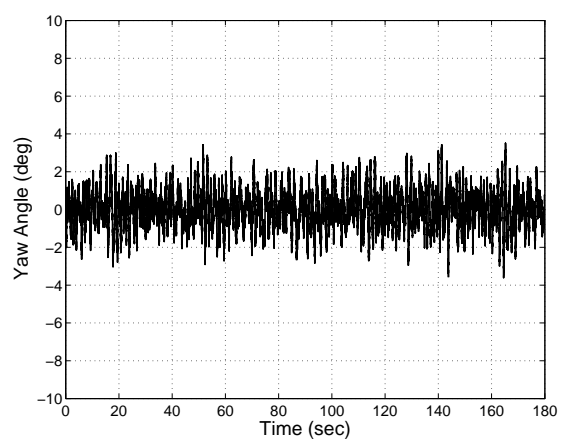

(b) Yaw angle for PID

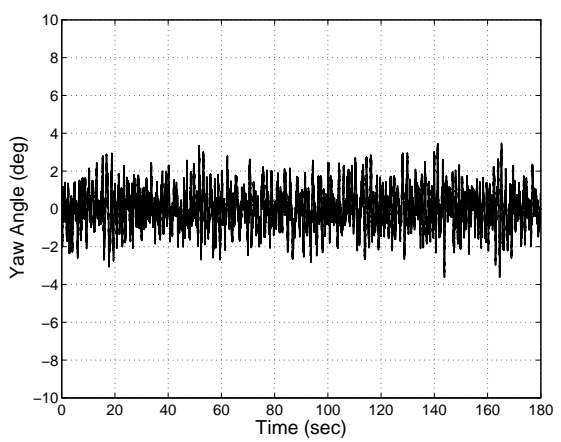

(d) Yaw angle for IB

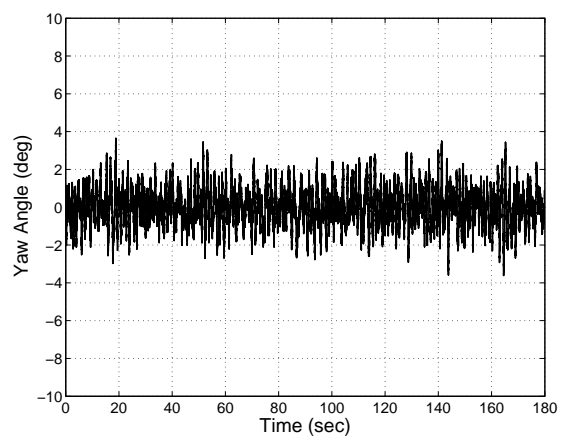

(f) Yaw angle for fuzzy

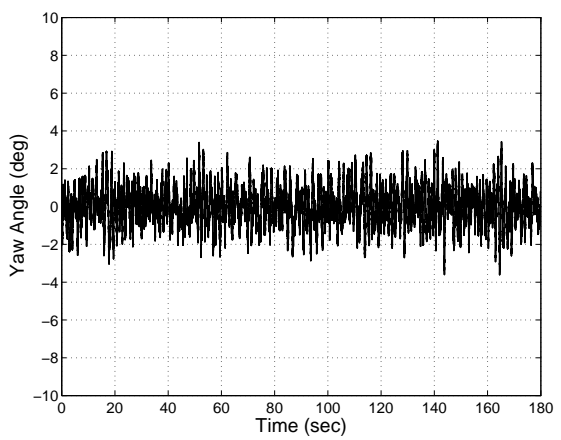

(h) Yaw angle for AIB

Figure 41: Z-axis step response. 
Table 11: Z-axis step response summary.

\begin{tabular}{|l|c|c|c|c|}
\cline { 2 - 5 } \multicolumn{1}{c|}{} & PID & IB & Fuzzy & AIB \\
\hline Undershoot (\%) & 0.0 & 0.0 & 0.0 & 0.0 \\
\hline Overshoot (\%) & 27.1 & 28.5 & 1.3 & 28.7 \\
\hline Rise Time (sec) & 2.9 & 2.7 & 5.7 & 2.7 \\
\hline Settling Time (sec) & 18.1 & 10.2 & 7.2 & 10.3 \\
\hline
\end{tabular}




\section{Chapter 5}

\section{Results}

The position control of TARA is simulated using PID, integral backstepping, fuzzy, and adaptive integral backstepping control techniques in a set of test flight cases, whereby each control technique's flight performance is evaluated under a consistent, waypoint based test flight lasting for twelve minutes, during which TARA is programmed to perform a one minute position hold at six pre-determined coordinates of interest, per the simulation methodology laid out in Section 3.3. The gains used here for each of the tested control approaches are those detailed in Chapter 4, obtained by tuning under no wind conditions. Appendix-C provides the sample results of the position hold flight simulation without wind at point-B.

A review based on the analysis of all the wind based simulations is summarized here, in order to assess the best performing control technique. The presented analysis also identifies certain wind disturbance characteristics and their effect on the position control performance. 


\subsection{Position Control Simulations in Wind}

\subsubsection{Dead Air Zone (Point-A)}

Figure 42 depicts the simulation results obtained for a 60 second position hold conducted at point-A for each of the PID, IB, fuzzy, and AIB controllers in the presence of wind. The error in position for the $\mathrm{X}, \mathrm{Y}$, and $\mathrm{Z}$ axes is plotted over the $60 \mathrm{~s}$ designated for holding point-A's coordinates during the full waypoint flight path from point-A to point-F. The corresponding flight performance metrics in terms of maximum error and AOAD are summarized in Table 12.

Point-A presents a dead air zone flight scenario close to the leeward side of the building. The mean steady state wind speeds for the X-axis and Y-axis are $-0.34 \mathrm{~m} / \mathrm{s}$ and $0.02 \mathrm{~m} / \mathrm{s}$, respectively, with turbulent erratic airflow. The Y-axis wind shows a greater range of fluctuations than that of the X-axis, with chaotic gusts of $\pm 3.2 \mathrm{~m} / \mathrm{s}$. Thus, a poorer position hold is anticipated for the Y-axis, as a result of this increased turbulence.

Of the four techniques considered, the PD controller delivers the best results for both the $\mathrm{X}$ and Y-axes, with AOAD values of $0.27 \mathrm{~m}$ and $0.53 \mathrm{~m}$, respectively. The maximum errors produced by the PD control are also the lowest ones achieved for this test case, at $0.98 \mathrm{~m}$ for the $\mathrm{X}$-axis and $1.65 \mathrm{~m}$ for the higher fluctuating winds of the $\mathrm{Y}$-axis. The $\mathrm{X}$-axis AOAD values of all four controllers fall within the "good" performance metric (error $\leq 1 \mathrm{~m}$ ), as defined in Table 2. For both the integral backstepping and AIB controllers the X-axis position control performance for this low mean chaotic airflow case results in the best AOAD for the X-axis of all the six test points.

As expected, the Y-axis wind presents a greater challenge to all four controllers, yielding correspondingly higher maximum error and AOAD values. As shown in Figure $42(\mathrm{~b})$, the AIB controller generates the highest maximum error and AOAD values, 
with a "poor" $(3<$ error $\leq 5) 3.71 \mathrm{~m}$ and an "acceptable" $(1<$ error $\leq 3) 1.27 \mathrm{~m}$, respectively. However, the AIB controller's motion is best centered about the designated hold location of all of the techniques. This ability of the AIB controller to bring the quadrotor's position back to the desired value in response to wind disturbances is reflected by calculating the mean error, yielding the lowest value of $0.26 \mathrm{~m}$. By contrast, the IB controller has a smaller maximum error $(2.55 \mathrm{~m})$ and AOAD (0.94 $\mathrm{m})$, but does not regulate the quadrotor's motion about the desired coordinates as well, with a mean error of $0.48 \mathrm{~m}$.

Generally, as illustrated in Figure 42(c), all four controllers produce comparable results for the Z-axis. The fuzzy controller has the lowest maximum error and AOAD for this axis, at $0.78 \mathrm{~m}$ and $0.24 \mathrm{~m}$, respectively.

Table 12: In wind control performance summary of position hold at point-A.

\begin{tabular}{|l|l|c|c|c|c|}
\cline { 3 - 6 } \multicolumn{2}{c|}{} & PD/PID & IB & Fuzzy & AIB \\
\hline \multirow{2}{*}{ X-axis } & Max. Error $(\mathrm{m})$ & 0.98 & 1.61 & 2.08 & 1.66 \\
\cline { 2 - 6 } & AOAD $(\mathrm{m})$ & 0.27 & 0.60 & 0.67 & 0.48 \\
\hline \hline \multirow{2}{*}{ Y-axis } & Max. Error $(\mathrm{m})$ & 1.65 & 2.55 & 2.96 & 3.71 \\
\cline { 2 - 6 } & AOAD $(\mathrm{m})$ & 0.53 & 0.94 & 1.07 & 1.27 \\
\hline \hline \multirow{2}{*}{ Z-axis } & Max. Error $(\mathrm{m})$ & 0.90 & 0.99 & 0.78 & 1.04 \\
\cline { 2 - 6 } & AOAD $(\mathrm{m})$ & 0.28 & 0.30 & 0.24 & 0.28 \\
\hline
\end{tabular}

\subsubsection{Passing Vortex (Point-B)}

The passing vortex scenario present at point-B exhibits high ranging, asymmetrical gusts on all three axes. As can be seen in Figure 43(a), the PD controller is unable to attain the desired hold position on the $\mathrm{X}$-axis, producing its worst result of all points A through F considered. Of the four controllers, the IB technique produces the best AOAD of $1.21 \mathrm{~m}$ (although, as summarized in Table 13, with large deviations 


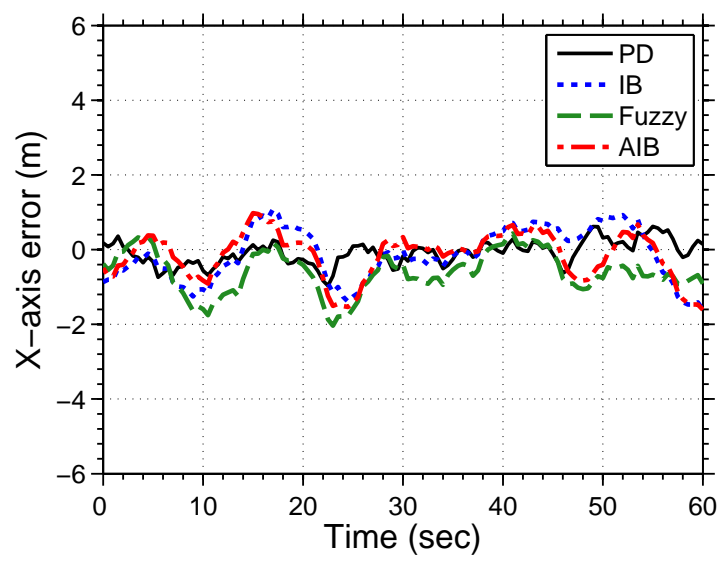

(a)

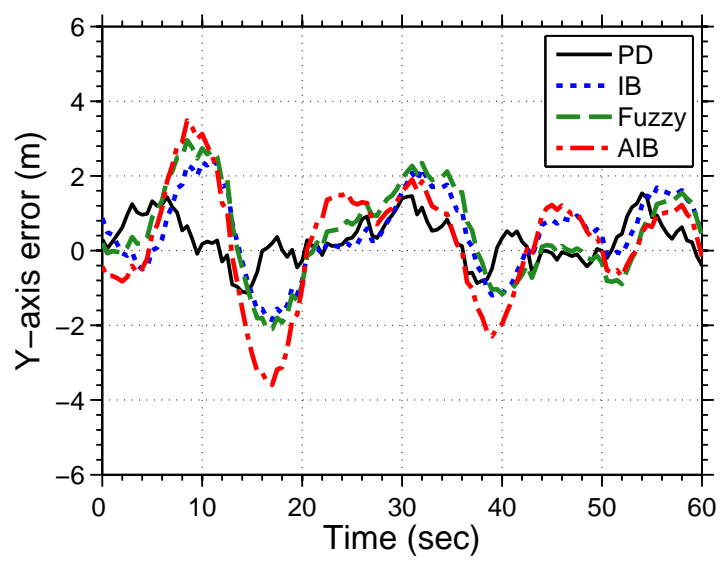

(b)

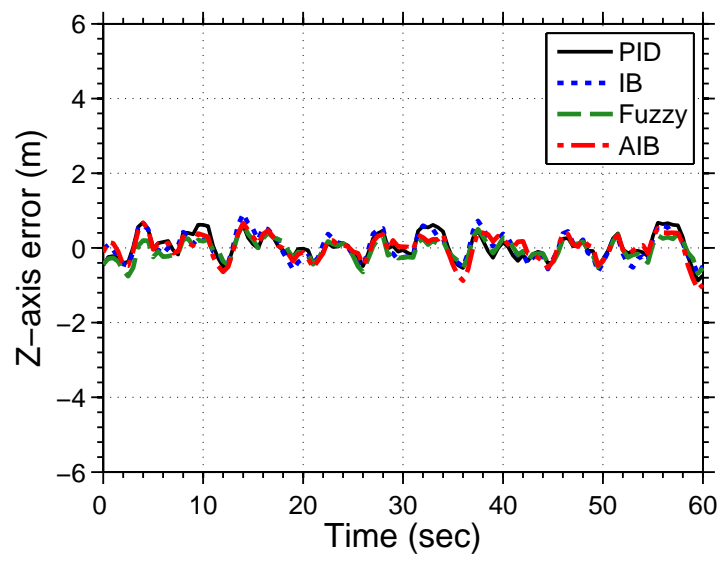

(c)

Figure 42: In wind position hold for dead air zone (point-A). (a) X-axis; (b) Y-axis; and (c) Z-axis.

producing errors of up to $3.92 \mathrm{~m}$ ). The AIB control has the next best AOAD value $1.47 \mathrm{~m}$, which still falls within the "acceptable" performance category $(1<$ error $\leq 3)$ even though it corresponds to the worst X-axis AOAD for the AIB controller. Despite generating slightly larger maximum error and AOAD values compared with the IB controller, the AIB technique proves best able to center the quadrotor's position towards the reference point, with a mean error of $0.28 \mathrm{~m}$, as opposed to the $0.66 \mathrm{~m}$ mean error calculated for the IB control.

Similarly, the Y-axis wind is best tolerated by the backstepping approach, with 
only the IB and AIB controllers evidencing a centralized hold. Again, the PD control has this axis' poorest AOAD $(1.86 \mathrm{~m})$, as the quadrotor's position is consistently close to $2 \mathrm{~m}$ away from point-B. The fuzzy control has its worst overall result (of all points $\mathrm{A}$ to $\mathrm{F}$ considered) with a maximum error of $4.94 \mathrm{~m}$ and AOAD of $1.63 \mathrm{~m}$. Figure 43(b) depicts the fuzzy control drifting away from the desired hold position before eventually reaching the specified reference point.

The Z-axis AOAD values obtained for each of the controllers fall within a couple of centimeters of each other. Only the maximum error demonstrated by the fuzzy control $(1.27 \mathrm{~m})$ lies beyond the defined bounds of a "good" performance (error $\leq 1$ $\mathrm{m})$.

Table 13: In wind control performance summary of position hold at Point-B.

\begin{tabular}{|l|l|c|c|c|c|}
\cline { 2 - 6 } \multicolumn{2}{c|}{} & PD/PID & IB & Fuzzy & AIB \\
\hline \multirow{2}{*}{ X-axis } & Max. Error $(\mathrm{m})$ & 5.99 & 3.92 & 3.96 & 4.12 \\
\cline { 2 - 6 } & AOAD $(\mathrm{m})$ & 4.18 & 1.21 & 1.41 & 1.47 \\
\hline \hline \multirow{2}{*}{ Y-axis } & Max. Error $(\mathrm{m})$ & 3.11 & 3.03 & 4.94 & 3.15 \\
\cline { 2 - 6 } & AOAD $(\mathrm{m})$ & 1.86 & 0.84 & 1.63 & 1.02 \\
\hline \hline \multirow{2}{*}{ Z-axis } & Max. Error $(\mathrm{m})$ & 0.88 & 0.91 & 1.27 & 0.81 \\
\cline { 2 - 6 } & AOAD $(\mathrm{m})$ & 0.26 & 0.20 & 0.24 & 0.22 \\
\hline
\end{tabular}

\subsubsection{Wake Eddy (Point-C)}

Compared with the high mean winds present for the passing vortex case on the $\mathrm{X}$-axis, the $\mathrm{X}$-axis winds for the wake eddy scenario are averaged close to $0 \mathrm{~m} / \mathrm{s}$ with gusts ranging from $-2.26 \mathrm{~m} / \mathrm{s}$ to $3.56 \mathrm{~m} / \mathrm{s}$. As compiled in Table 14, the simulation results reflect this distinction, with all of the tested controllers evidencing better performance in terms of maximum error and AOAD. In particular, the PD control shows a marked improvement with the best $\operatorname{AOAD}(0.67 \mathrm{~m})$ and maximum error $(1.87 \mathrm{~m})$ for this 


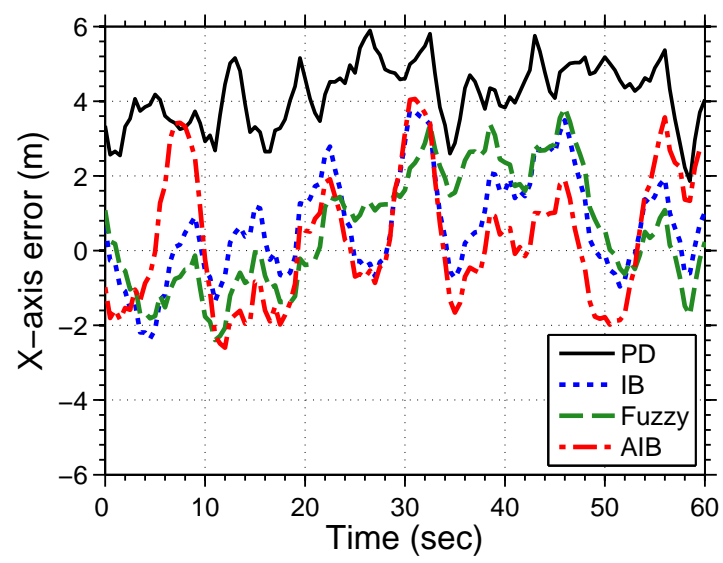

(a)

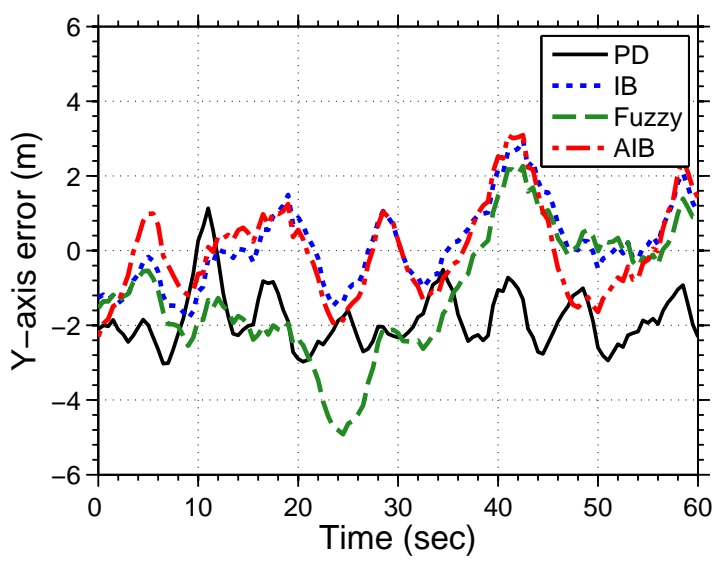

(b)

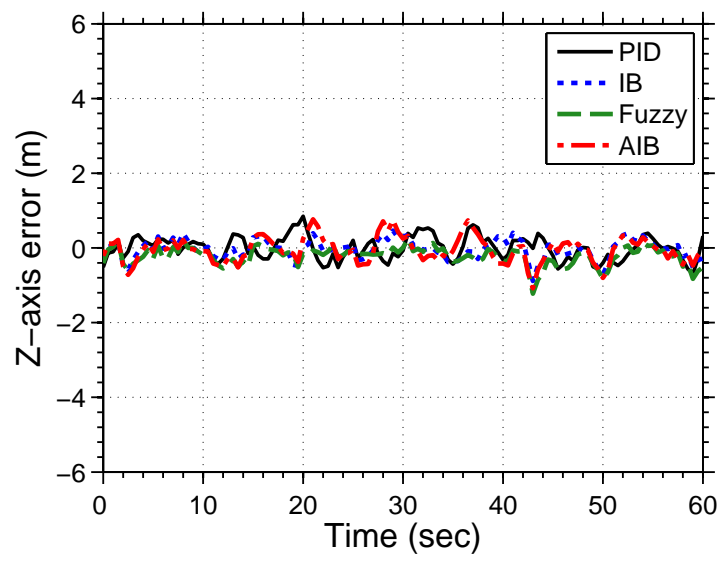

(c)

Figure 43: In wind position hold for passing vortex (Point-B). (a) X-axis; (b) Y-axis; and (c) Z-axis.

axis.

The Y-axis winds in the wake eddy have the largest range of any of the test cases, varying between $-4.55 \mathrm{~m} / \mathrm{s}$ to $4.04 \mathrm{~m} / \mathrm{s}$. Here the PD controller has the lowest maximum error $(1.94 \mathrm{~m})$, with a comparable AOAD value for the AIB controller, at $0.75 \mathrm{~m}$ and $0.80 \mathrm{~m}$, respectively. The IB, fuzzy and AIB controllers all show better performance for the $\mathrm{Y}$-axis than for the $\mathrm{X}$-axis, despite the higher ranging winds in the former instance. The better $\mathrm{Y}$-axis performance is due to the fact that the gust disturbances present here are evenly distributed across the Y-axis. 
All four controllers have their overall worst altitude performance for this case, which features the largest ranging Z-axis wind and an average velocity of $-0.88 \mathrm{~m} / \mathrm{s}$. As illustrated in Figure 44(c), all the controllers allow for maximum errors which can be characterized as "acceptable" $(1<$ error $\leq 3 \mathrm{~m})$. The fuzzy controller attains the tightest hold, with the PID controller producing the largest errors.

Table 14: In wind control performance summary of position hold at point-C.

\begin{tabular}{|l|l|c|c|c|c|}
\cline { 2 - 6 } \multicolumn{2}{c|}{} & PD/PID & IB & Fuzzy & AIB \\
\hline \multirow{2}{*}{ X-axis } & Max. Error $(\mathrm{m})$ & 1.87 & 3.03 & 3.42 & 4.00 \\
\cline { 2 - 6 } & AOAD $(\mathrm{m})$ & 0.67 & 1.06 & 1.22 & 1.17 \\
\hline \hline \multirow{2}{*}{ Y-axis } & Max. Error $(\mathrm{m})$ & 1.94 & 2.35 & 3.08 & 2.23 \\
\cline { 2 - 6 } & AOAD $(\mathrm{m})$ & 0.75 & 1.07 & 1.16 & 0.80 \\
\hline \hline \multirow{2}{*}{ Z-axis } & Max. Error $(\mathrm{m})$ & 1.96 & 1.68 & 1.52 & 1.78 \\
\cline { 2 - 6 } & AOAD $(\mathrm{m})$ & 0.55 & 0.53 & 0.44 & 0.48 \\
\hline
\end{tabular}

\subsubsection{Downwash (Point-D)}

Table 15 and Figure 45 highlight the outcomes of the flight controllers' performance in the downwash region. The $\mathrm{X}$-axis gusts are the only example considered of a turbulent wind without reversals of flow. The observed velocities are all positive, ranging between $0.42 \mathrm{~m} / \mathrm{s}$ and $4.43 \mathrm{~m} / \mathrm{s}$, with the preponderance of the wind disproportionately concentrated towards the higher velocities. The PD controller has the worst AOAD of this case $(1.85 \mathrm{~m})$, but the best maximum error $(2.85 \mathrm{~m})$. The AIB controller does the best job of bringing the quadrotor's position back towards the desired value, with the lowest $\mathrm{AOAD}$ value at $1.09 \mathrm{~m}$. Here the IB controller has its worst maximum error and AOAD performance over all the test cases. The fuzzy controller also has its worst X-axis result. Notably, the position obtained by the PD and fuzzy controllers here is of an overdamped nature and is consistently laterally biased to one 


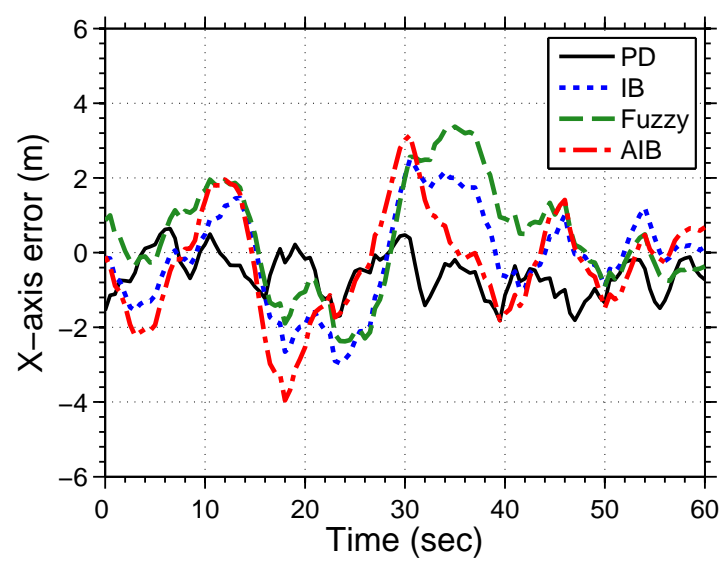

(a)

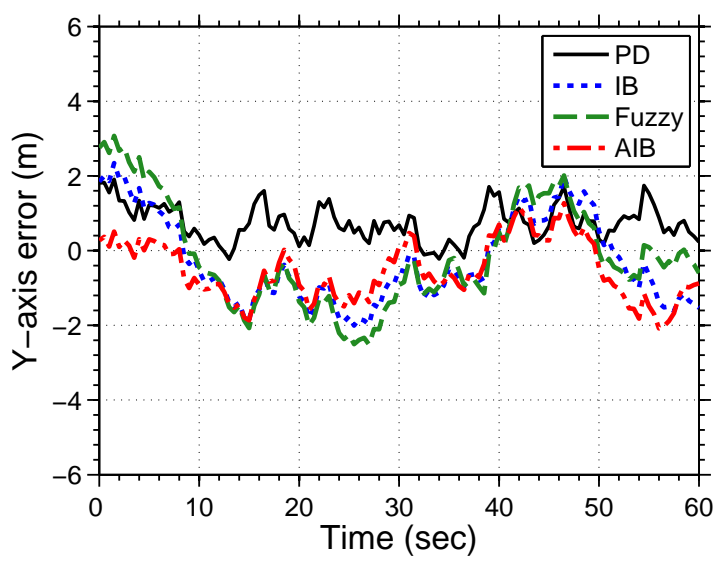

(b)

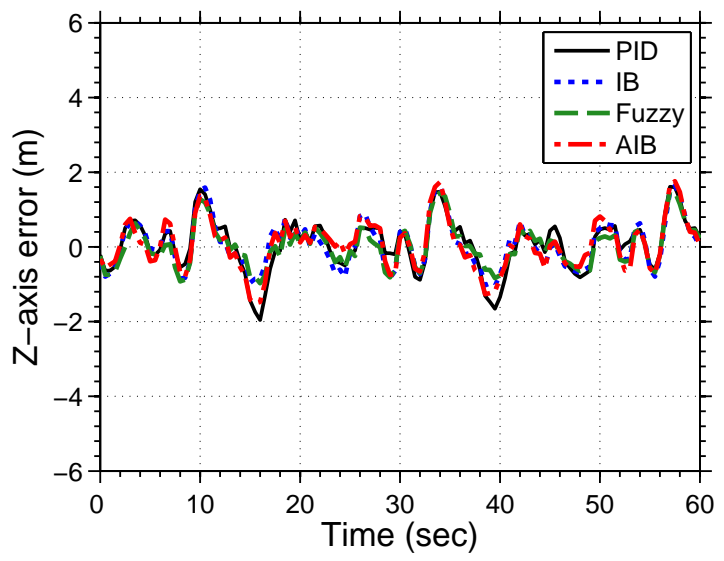

(c)

Figure 44: In wind position hold for wake eddy (point-C). (a) X-axis; (b) Y-axis; and (c) Z-axis.

side of the reference point, as shown in Figure 45(a).

Of all the wind conditions considered in the test case simulations, the Y-axis wind at point-D exhibits variability in the form of pronounced extremes, alternating close to its $0 \mathrm{~m} / \mathrm{s}$ mean with occasional isolated wind gusts spiking up to $-2.85 \mathrm{~m} / \mathrm{s}$. Hence, the significance of this particular test lies in its presentation of impulse type perturbations to the quadrotor, affording the opportunity to verify the controller's performance in rejecting disturbances of this nature. Indeed, all four tested control techniques are able to maintain a "good" AOAD, demonstrating an adequate response 
to the gusts. In a clear illustration of disturbance rejection, the PD control provides a solid hold and evidences the smallest deviations in response to the spikes in velocity, as depicted in Figure 45(b). The IB controller shows the largest divergence, with a maximum error of $2.98 \mathrm{~m}$ and AOAD of $0.81 \mathrm{~m}$. Both the IB and fuzzy controllers demonstrate a slow regulatory response, taking a long time to bring the quadrotor's position back to the set point.

The Z-axis wind at point-D has the highest mean velocity $(-1.0 \mathrm{~m} / \mathrm{s})$, but the smallest ranging gusts $(-2.2 \mathrm{~m} / \mathrm{s}$ to $1.2 \mathrm{~m} / \mathrm{s})$ of any disturbance encountered thus far on this axis. Compared with point-C, the milder gust intensity corresponds with improved performance results for this case. The PID controller has slightly elevated maximum error $(0.12 \mathrm{~m})$ and $\operatorname{AOAD}(0.40 \mathrm{~m})$ values compared with the other controllers. In general, it can be observed that the Z-axis produces superior position hold performance over the $\mathrm{X}$-axis and $\mathrm{Y}$-axis, due to the presence of gravitational forces acting as a damping for the counter thrust force, helping in altitude stabilization.

Table 15: In wind control performance summary of position hold at point-D.

\begin{tabular}{|l|l|c|c|c|c|}
\cline { 2 - 6 } \multicolumn{2}{c|}{} & PD/PID & IB & Fuzzy & AIB \\
\hline \multirow{2}{*}{ X-axis } & Max. Error $(\mathrm{m})$ & 2.85 & 5.17 & 4.09 & 4.17 \\
\cline { 2 - 6 } & AOAD $(\mathrm{m})$ & 1.85 & 1.34 & 1.49 & 1.09 \\
\hline \hline \multirow{2}{*}{ Y-axis } & Max. Error $(\mathrm{m})$ & 0.86 & 2.98 & 1.61 & 2.24 \\
\cline { 2 - 6 } & AOAD $(\mathrm{m})$ & 0.19 & 0.81 & 0.56 & 0.62 \\
\hline \hline \multirow{2}{*}{ Z-axis } & Max. Error $(\mathrm{m})$ & 0.40 & 0.29 & 0.31 & 0.27 \\
\cline { 2 - 6 } & AOAD $(\mathrm{m})$ & 0.12 & 0.07 & 0.08 & 0.07 \\
\hline
\end{tabular}

\subsubsection{Accelerated Freestream (Point-E)}

The $4.51 \mathrm{~m} / \mathrm{s}$ accelerated freestream wind present on the $\mathrm{X}$-axis at point-E has the highest mean velocity encountered over the course of the simulations. As summarized 


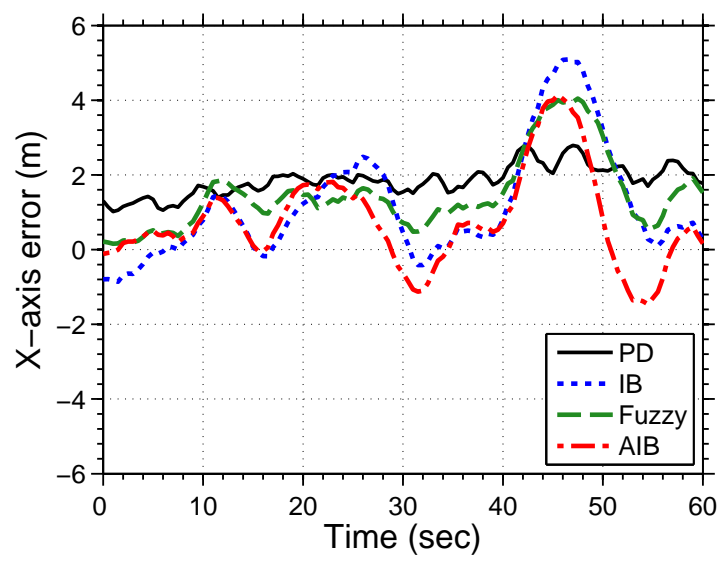

(a)

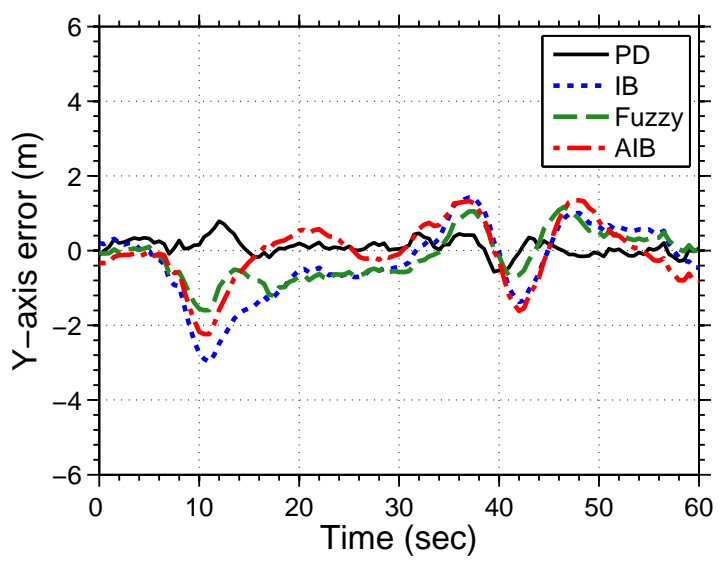

(b)

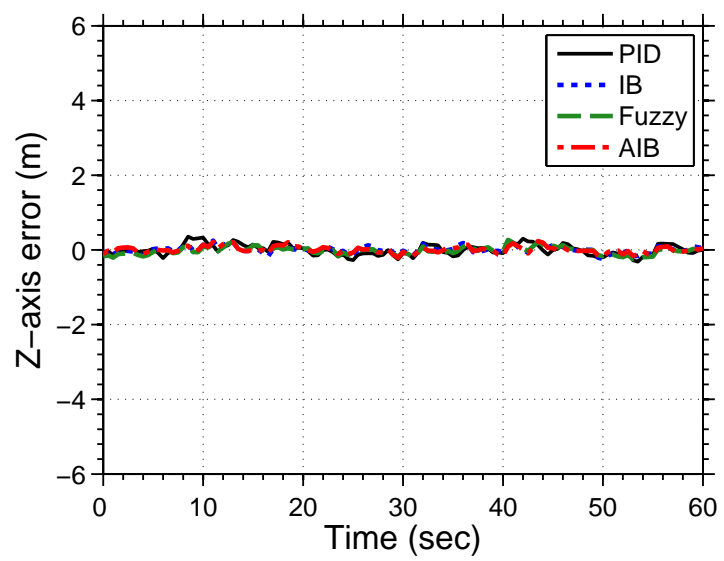

(c)

Figure 45: In wind position hold for downwash (point-D). (a) X-axis; (b) Y-axis; and (c) Z-axis.

in Table 16, the PD controller has a maximum error $(0.33 \mathrm{~m})$ and $\operatorname{AOAD}(0.09 \mathrm{~m})$ results, representing its best performance for both the $\mathrm{X}$ and $\mathrm{Y}$-axes of all of the test cases. As depicted in Figure 46(a) only the PD and AIB controllers are able to center the quadrotor's motion about the reference point. The IB controller has the worst maximum error and AOAD values, at $2.16 \mathrm{~m}$ and $0.98 \mathrm{~m}$, respectively.

Figure 46(b) illustrates the position hold results for the Y-axis for the accelerated freestream. All of the controllers show greater errors with the low frequency, low intensity Y-axis winds, as compared with the $\mathrm{X}$-axis result. The PD controller 
produces the best performance here, with the AIB controller experiencing its overall worst $\operatorname{AOAD}(1.33 \mathrm{~m})$ at point-E, with a "poor" $(3<$ error $\leq 5)$ maximum error of $3.18 \mathrm{~m}$.

The Z-axis results can be seen in Figure 46(c). All of the controllers provide a "good" performance (error $\leq 1 \mathrm{~m})$, by the established metric, with the AIB and IB controller producing identical AOADs of $0.08 \mathrm{~m}$.

Table 16: In wind control performance summary of position hold at point-E.

\begin{tabular}{|l|l|c|c|c|c|}
\cline { 2 - 6 } \multicolumn{2}{c|}{} & PD/PID & IB & Fuzzy & AIB \\
\hline \multirow{2}{*}{ X-axis } & Max. Error $(\mathrm{m})$ & 0.33 & 2.16 & 1.39 & 1.20 \\
\cline { 2 - 6 } & AOAD $(\mathrm{m})$ & 0.19 & 0.98 & 0.76 & 0.48 \\
\hline \hline \multirow{2}{*}{ Y-axis } & Max. Error $(\mathrm{m})$ & 1.03 & 3.32 & 2.74 & 3.18 \\
\cline { 2 - 6 } & AOAD $(\mathrm{m})$ & 0.34 & 1.12 & 0.95 & 1.33 \\
\hline \hline \multirow{2}{*}{ Z-axis } & Max. Error $(\mathrm{m})$ & 0.38 & 0.31 & 0.59 & 0.31 \\
\cline { 2 - 6 } & AOAD $(\mathrm{m})$ & 0.10 & 0.08 & 0.14 & 0.08 \\
\hline
\end{tabular}

\subsubsection{Freestream Wind (Point-F)}

Point-F is the freestream wind test case, with a mean $3.63 \mathrm{~m} / \mathrm{s}$ wind present on the X-axis. Table 17 and Figure 47(a) recap the simulated flight performance obtained in this scenario. The PD controller has a very good result, producing an AOAD of $0.19 \mathrm{~m}$. The AIB controller also has a "good" AOAD $(0.87 \mathrm{~m})$ and maintains a hold centered about the desired location. By contrast, the fuzzy controller is not able to bring the quadrotor's position to the desired coordinates. The IB control also has a "poor" $(3<$ error $\leq 5)$ maximum error of $3.17 \mathrm{~m}$.

The Y-axis results, shown in Figure 47(b) once again highlight the PD controller's superiority in freestream conditions. However, all of the controllers evidenced a "good" AOAD value for this axis, with "acceptable" $(1<$ error $\leq 3)$ rated maximum 


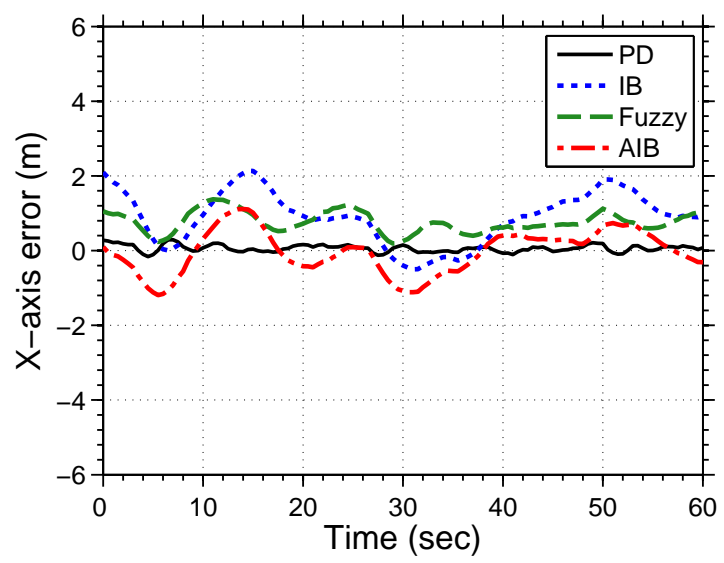

(a)

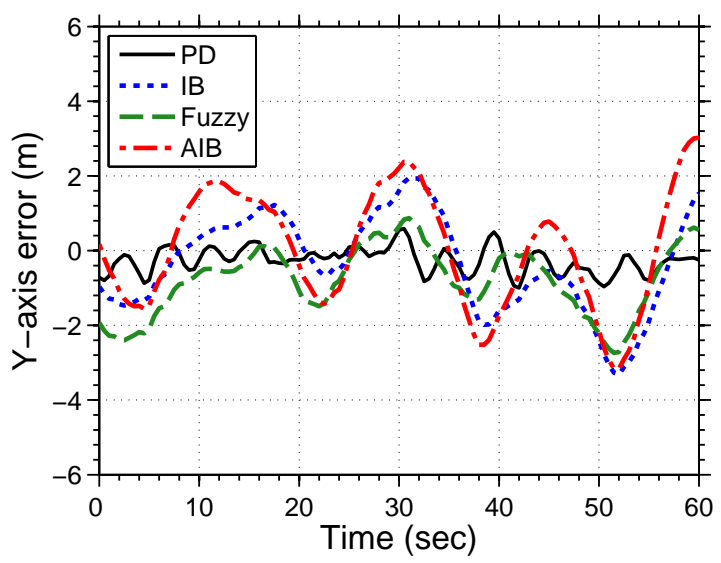

(b)

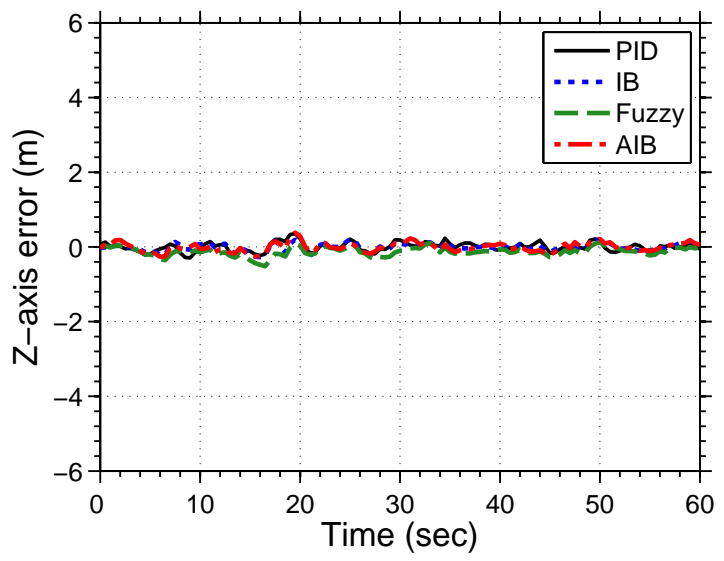

(c)

Figure 46: In wind position hold for accelerated freestream (point-E). (a) X-axis; (b) Y-axis; and (c) Z-axis.

errors. On the whole, compared to the accelerated freestream results, the Y-axis hold shows improvement at point-F, while the $\mathrm{X}$-axis hold is marginally better at point-E.

The altitude hold performance in the freestream, depicted in Figure 47(c), produced comparably strong hold results for all four controllers. The overall best simulation AOAD values are obtained for the PID, IB, and AIB controllers here. In fact, the AOAD of the IB controller indicates an average altitude error of $3 \mathrm{~cm}$. 
Table 17: In wind control performance summary of position hold at point-F.

\begin{tabular}{|l|l|c|c|c|c|}
\cline { 2 - 6 } \multicolumn{2}{c|}{} & PD/PID & IB & Fuzzy & AIB \\
\hline \multirow{2}{*}{ X-axis } & Max. Error $(\mathrm{m})$ & 0.78 & 3.17 & 1.67 & 2.21 \\
\cline { 2 - 6 } & AOAD $(\mathrm{m})$ & 0.19 & 1.08 & 0.72 & 0.87 \\
\hline \hline \multirow{2}{*}{ Y-axis } & Max. Error $(\mathrm{m})$ & 1.17 & 2.27 & 1.34 & 1.88 \\
\cline { 2 - 6 } & AOAD $(\mathrm{m})$ & 0.44 & 0.81 & 0.45 & 0.84 \\
\hline \hline \multirow{2}{*}{ Z-axis } & Max. Error $(\mathrm{m})$ & 0.32 & 0.13 & 0.30 & 0.13 \\
\cline { 2 - 6 } & AOAD $(\mathrm{m})$ & 0.08 & 0.03 & 0.12 & 0.04 \\
\hline
\end{tabular}

\subsection{Review}

Over the full course of test case simulations, the unstated gross objective of TARA not hitting the building is successfully attained. Yet, of the four control techniques investigated thus far, no control design performs to such a standard as to warrant designating it as the single preferred quadrotor position controller in the urban wind environment. In the no wind simulations, the PID, IB, fuzzy and AIB controls all achieve satisfactory position hold, rarely surpassing the defined bounds of a "good" (error $\leq 1$ or within 2 body lengths) performance. However, in conducting the same test scenarios in the wake of a building with wind disturbances present, disparities arise in the effectiveness of each technique in regulating TARA's position. Generally, the fuzzy controller demonstrates a slow regulatory response and has difficulties in maintaining the desired hold position as the mean speed of the wind increases, occasionally not attaining the designated coordinates. Still, for the less perturbed Z-axis, the fuzzy controller a few times produces the best AOAD of any of the techniques investigated. On the other hand, the integral backstepping control is habitually able to bring the quadrotor's position to the target value, particularly in the higher wind cases where the fuzzy controller struggles, even if the desired position is not consistently maintained. For the higher wind cases, the cost of establishing a more centered hold is 


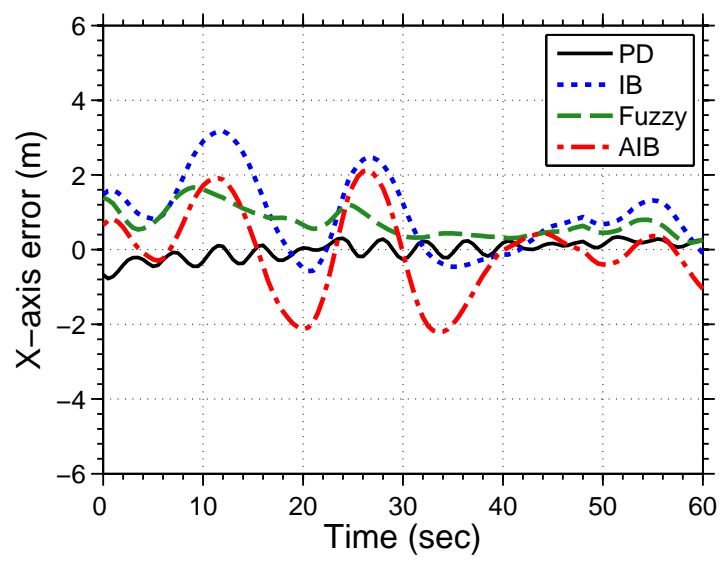

(a)

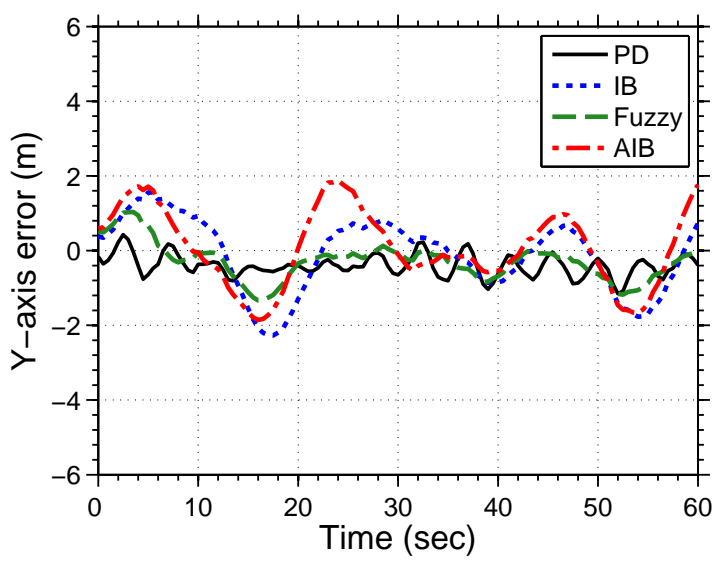

(b)

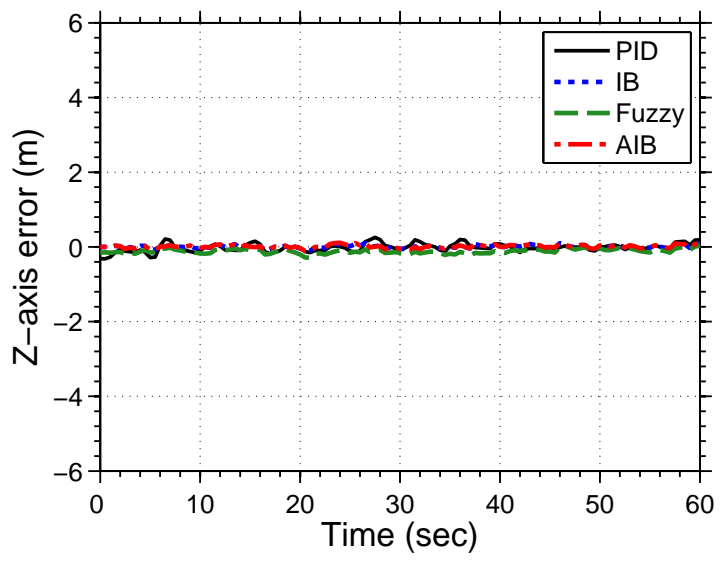

(c)

Figure 47: In wind position hold for freestream wind (point-F). (a) X-axis; (b) Y-axis; and (c) Z-axis.

reflected in slightly elevated maximum error values for the IB control, when compared with the fuzzy controller.

Over the full course of the simulations the PD/PID controller most often produces the least maximum error and lowest AOAD of all of the techniques considered. However, under certain wind conditions characterized by turbulent asymmetrical gusts, the PD controller produces the largest AOAD of all of the controllers. For the $\mathrm{X}$ and $\mathrm{Y}$-axes at point-B, and the $\mathrm{X}$-axis at point-D, the PD controller is not able to converge TARA's position towards the target hover location, essentially producing a 
steady state error condition. Interestingly, the single largest maximum error observed in all of the test cases $(5.99 \mathrm{~m})$ occurred in the PD controller simulation at point-B (passing vortex) along the $\mathrm{X}$-axis.

By contrast, the AIB controller proves to be the only control method which always converges the quadrotor's position towards the desired location for all three axes in each test case. Notably, even for the high wind cases, the AIB response is well regulated about the reference set point, without the offset present in the other controller simulations. Although the AIB controller achieves the best overall AOAD for a few test cases, the maximum error and AOAD data produced typically tend to be mid-range in comparison with the other control techniques, with a tendency towards slightly elevated maximum errors. At point-A and point-B it can be noted that the AOAD and maximum error values of the IB controller are smaller than for its adaptive counterpart. However, the AIB technique shows better centering of the quadrotor's position around the desired coordinates, as reflected in lower mean error values. Otherwise, the maximum error and AOAD performance obtained by the AIB controller is comparable to or better than that of the IB controller.

\subsection{Experimental Flight Results}

Experimental flight results are presented in this section to validate the control performances of the concerned control techniques.

\subsubsection{PD/PID based Indoor Position Control (No Wind)}

A flight simulation of position control is performed and repeated in an experimental flight test indoors to evaluate the PD/PID controllers developed in the previous sections. Shown in Figure 48, during the flight tests sonar sensors are used for measuring ranges from the right $(+\mathrm{Y}$-axis) and back (-X-axis) walls, as well as the height 
(Z-axis) in a squash court of length $9.50 \mathrm{~m}$ and width of $6.50 \mathrm{~m}$. The yaw angle controller is such that it records the initial heading of TARA at the start time and during flight works to maintain this initial heading angle. A total flight time of three minutes is allowed for the test results presented.

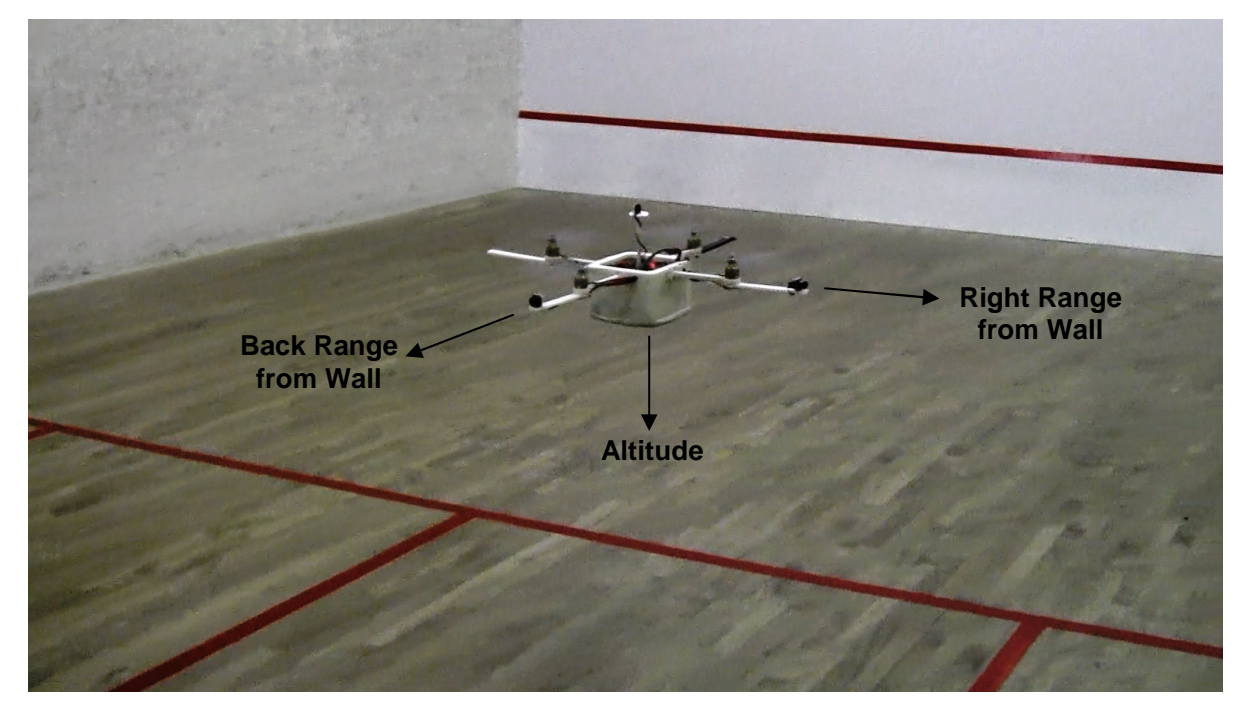

Figure 48: Experimental indoor flight test setup.

Indoor flight tests are conducted to compare the performance under zero wind conditions. In the flight setup TARA is placed with a right side distance of $2 \mathrm{~m}$ from the right wall and a back side distance of $2 \mathrm{~m}$ from the back wall, set as its initial position. The desired distance to maintain from the walls is $2 \mathrm{~m}$ and the desired altitude is $0.5 \mathrm{~m}$. A radio control input is used to provide enough throttle for TARA to takeoff from the ground and to provide a safety override during the flight tests.

The X-axis and Y-axis control performances are shown in Figures 49 and 50, respectively. The PD controllers are able to achieve a "good" lateral position control performance according to the established criteria defined in Table 2. For the X-axis data, an experimental AOAD of $0.33 \mathrm{~m}$ is calculated, neglecting the sonar glitches manifesting as spikes in error, as indicated by the red dashed lines (refer to AppendixB for details on sonar glitches) in Figure 49. A corresponding simulation AOAD of 
$0.06 \mathrm{~m}$ is recorded for this case. An experimental flight AOAD of $0.16 \mathrm{~m}$ is obtained for the Y-axis, compared with the simulation AOAD of $0.08 \mathrm{~m}$. As simulations are performed in the complete absence of wind disturbances, the results of the simulation show more optimistic values when compared with indoor flight, where unmodelled ground effects come into play at an altitude of $0.5 \mathrm{~m}$.

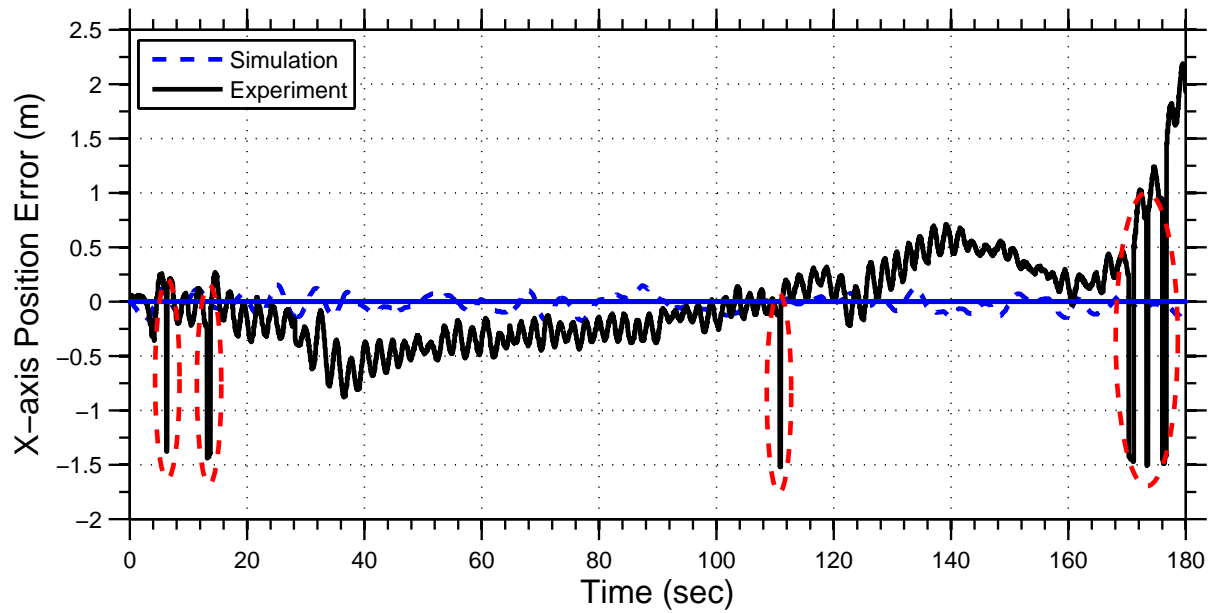

Figure 49: Indoor flight test: $\mathrm{X}$-axis position error.

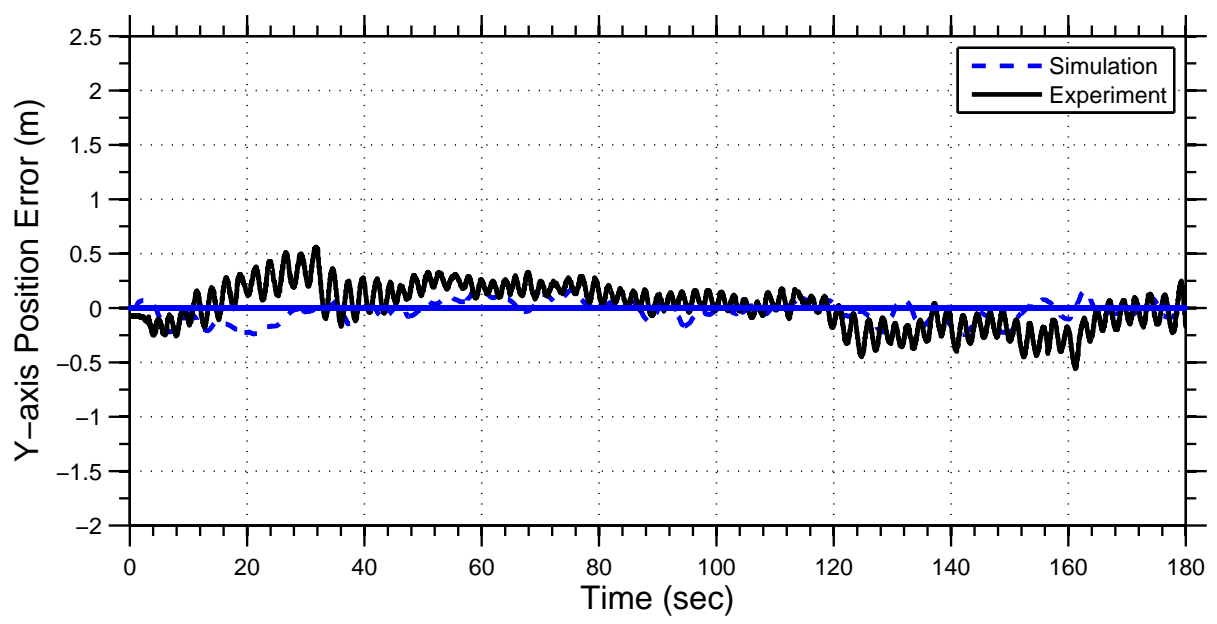

Figure 50: Indoor flight test: Y-axis position error.

Figure 51 shows the altitude data for the indoor PID flight. An overshoot of 0.66 $\mathrm{m}$ is observed close to takeoff in the experimental data, with a settling time of 20 seconds shown in both the experimental and simulation flights. The experimental 
altitude settles below the desired value, with a "good" AOAD of $0.28 \mathrm{~m}$, compared with simulation AOAD of $0.03 \mathrm{~m}$.

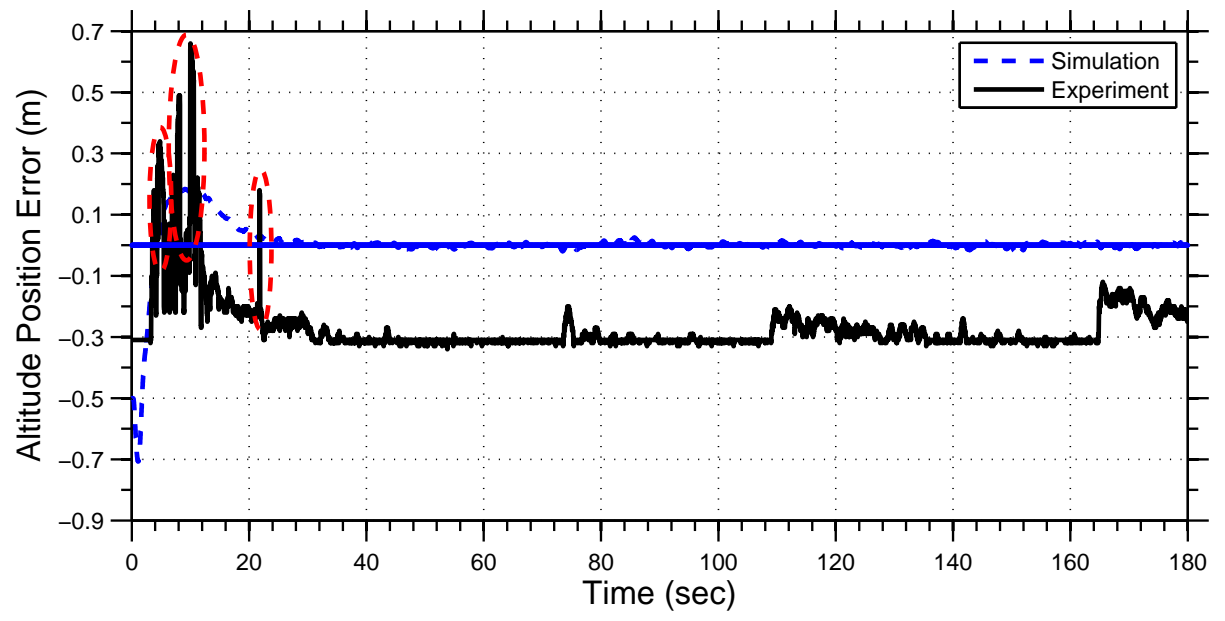

Figure 51: Indoor flight test: Altitude control.

\subsubsection{PD/PID-based Outdoor Position Control}

Outdoor experimental flights are conducted on the rooftop of a six storey high parking garage at Carleton University, in a setup depicted in Figure 52. The building is located such that the roof is exposed to incoming winds from all directions without hindrance. An ideally-located stairwell in the center of the roof is used as a wake producing cube-shaped building. The dimensions of the stairwell are $L=4.9 \mathrm{~m}, W=3.4 \mathrm{~m}$, and $H=2.6 \mathrm{~m}$. A $9 \times 3 \mathrm{~m}$ wall is constructed using an ABS piping frame with a construction tarp stretched across. The wall is placed next to the stairwell such that a L-shaped corner is formed. As TARA uses sonar range sensors for centimetre accuracy in positioning with respect to objects, the stairwell and erected tarp walls are used as a reference for positioning TARA such that it flies in the wake of the stairwell. TARA is programmed to maintain a $3 \times 3 \mathrm{~m}$ distance from the walls and a desired altitude of $0.75 \mathrm{~m}$ to avoid ground effects. A weather station is placed on the roof edge of the stairwell to take localized measurements of the incoming wind 
speed and direction. General wind conditions reported by the weather station at the Ottawa airport are consulted beforehand in order to plan for a suitable day and time for a flight test. TARA is placed such that the back sonar (X-axis) points towards the stairwell wall and the right sonar (Y-axis) points at the tarp wall, and the initial position is set as $3 \mathrm{~m}$ from each wall. The desired position hold coordinates are $X=3, Y=3$, and $Z=0.75 \mathrm{~m}$. Throughout the flight test a manual override is kept ready for immediate landing and disarming the motors. The flight data is recorded via XBee telemetry.

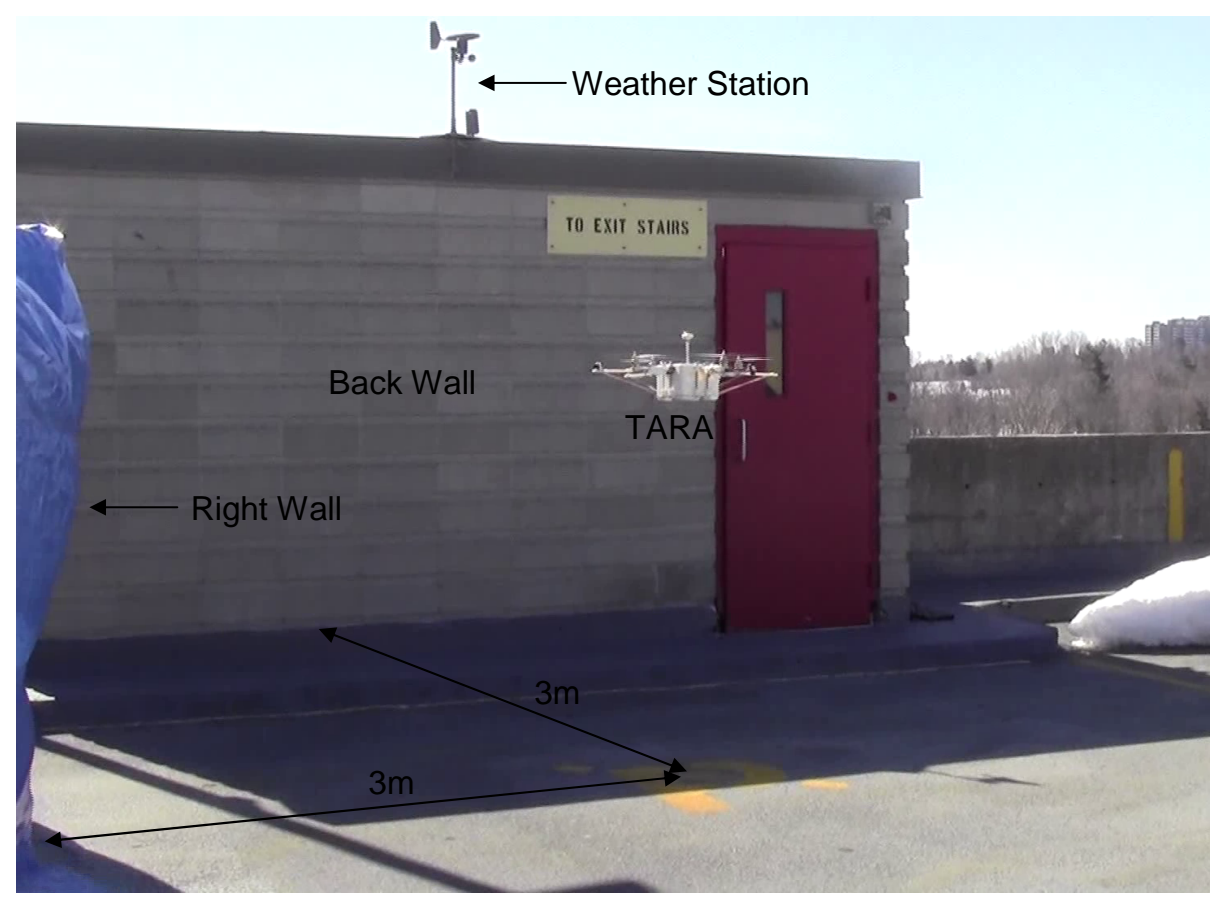

Figure 52: Experimental outdoor flight test setup.

On the day selected for conducting the flight test, the weather forecast called for an average wind speed of $4 \mathrm{~m} / \mathrm{s}$ (Beaufort scale value $=3$ ) from the south east with gusts up to $6 \mathrm{~m} / \mathrm{s}$. The weather station unit placed on the stairwell roof recorded wind speeds of $1.1 \mathrm{~m} / \mathrm{s}$ and $1.7 \mathrm{~m} / \mathrm{s}$ during the flight test; however, the station's 2 minute update rate is inadequate to fully characterize the freestream wind on the duration of the flight. Based on the recorded wind velocity and the dimensions of 
the stairwell the Reynold's number is calculated as $R_{e}=6.8 \times 10^{5}$, as compared to the Reynold's number $R_{e}=7.3 \times 10^{6}$ for the wind simulations. Reviewing the weather vane orientation in the flight video, significant gusts or directional shifts are evident at approximately 3, 8, 17, 37, and 53 seconds into the flight. The varying wind direction shown by the weather vane is indicated at 5 second intervals for both the $\mathrm{X}$ and $\mathrm{Y}$-axes in Figures 53(a) and 53(c), respectively.

The outdoor flight results obtained for TARA using PD/PID position control are presented in Figure 53. Table 18 summarizes the performance metrics obtained for the angles and the position. Generally, as compared with the indoor results for PD/PID, greater maximum error and AOAD values are evident for the outdoor flight. The $\mathrm{X}$-axis position shows the most deviation with a "poor" $(3<$ error $\leq 5)$ maximum error of $3.03 \mathrm{~m}$, and an "acceptable" AOAD of $1.21 \mathrm{~m}$. As depicted in Figure 53(a), the control takes more than 30 seconds to direct TARA back to the desired setpoint following its initial deviation. The $\mathrm{Y}$-axis shows sonar artifacts (refer to Appendix-B for details on sonar glitches) at $\mathrm{t}=42$ and $\mathrm{t}=55 \mathrm{sec}$, as indicated in Figure 53(c). With these spurious data spikes excluded, the maximum error for the Y-axis is 1.18 $\mathrm{m}$ and a "good" AOAD of $0.47 \mathrm{~m}$ is obtained. The $0.32 \mathrm{~m}$ AOAD for the Z-axis is comparable to its indoor no wind counterpart.

The roll and pitch angles stay within the "good" performance metric for AOAD, with values of 1.70 and 2.14 degrees, respectively. Substantial yaw deviations up to 17.00 degrees are recorded. The yaw results reflect the fact that at times when the control action results in a higher demand of current, electromagnetic interference from the power lines effects the magnetometer measurements, causing an offset to be added to the actual heading. The yaw controller attempts to erroneously correct the yaw in response to what it considers to be a change in heading.

In order to benchmark this experimental flight performance with the simulated flight tests, the highest maximum error and average AOAD values are obtained over 
Table 18: PID outdoor position control summary.

\begin{tabular}{|l|c|c|c||c|c|c|}
\cline { 2 - 7 } \multicolumn{1}{c|}{} & \multicolumn{3}{c||}{ Angles (deg) } & \multicolumn{3}{c|}{ Position (m) } \\
\cline { 2 - 7 } \multicolumn{1}{c|}{} & Roll & Pitch & Yaw & X-axis & Y-axis & Z-axis \\
\hline Max. Error & 6.30 & 9.20 & 17.00 & 3.03 & 2.51 & 0.55 \\
\hline AOAD & 1.70 & 2.14 & 5.82 & 1.21 & 0.47 & 0.32 \\
\hline
\end{tabular}

the course of the simulations for point-A through point-F as listed in Table 19. The AOAD values correspond to a great degree with a difference of $0.00 \mathrm{~m}, 0.22 \mathrm{~m}$, and $0.09 \mathrm{~m}$ for the $\mathrm{X}, \mathrm{Y}$, and $\mathrm{Z}$ axes respectively. The experimental maximum error results are bounded by the simulation highest maximum error values of $5.99 \mathrm{~m}, 3.11 \mathrm{~m}$, and $1.96 \mathrm{~m}$ for the $\mathrm{X}, \mathrm{Y}$, and $\mathrm{Z}$ axes respectively.

Table 19: PD/PID simulation position hold summary over all points A through F.

\begin{tabular}{|l|c|c|c|}
\cline { 2 - 4 } \multicolumn{1}{c|}{} & X-axis & Y-axis & Z-axis \\
\hline Highest Max. Error $(\mathrm{m})$ & 5.99 & 3.11 & 1.96 \\
\hline Average AOAD $(\mathrm{m})$ & 1.21 & 0.69 & 0.23 \\
\hline
\end{tabular}




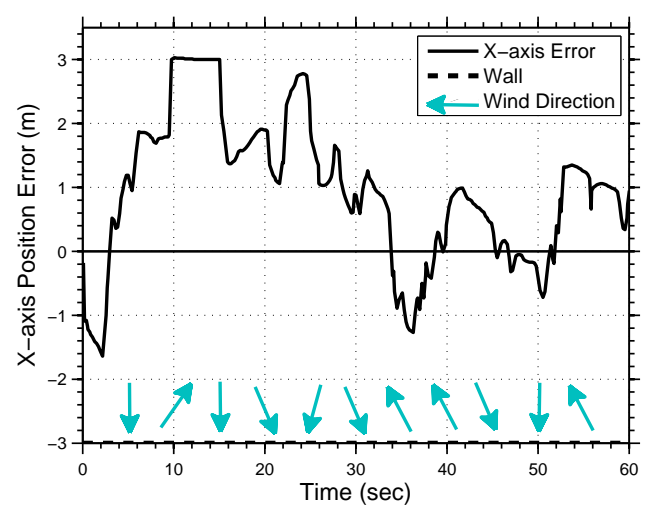

(a)

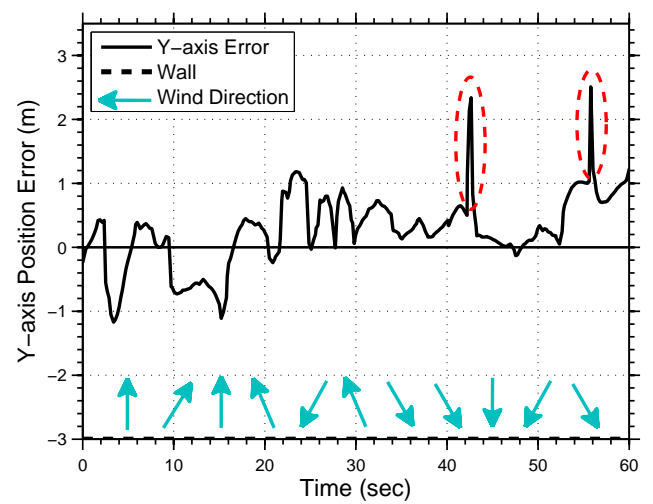

(c)

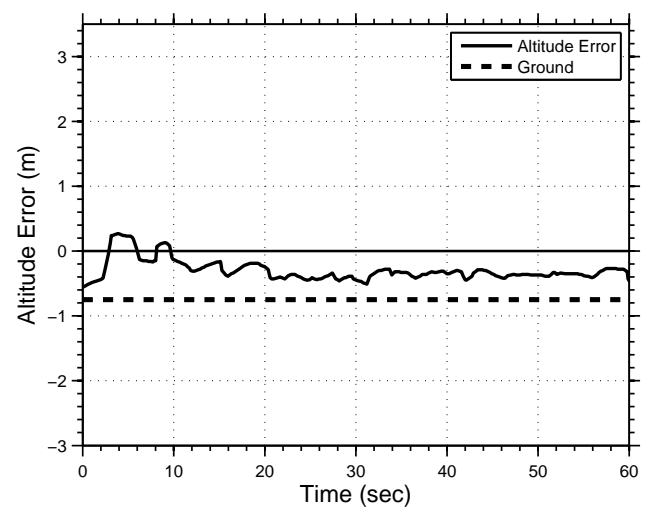

(e)

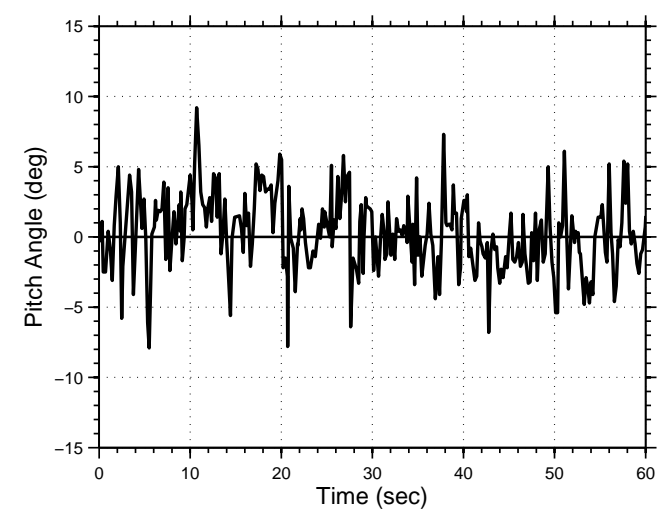

(b)

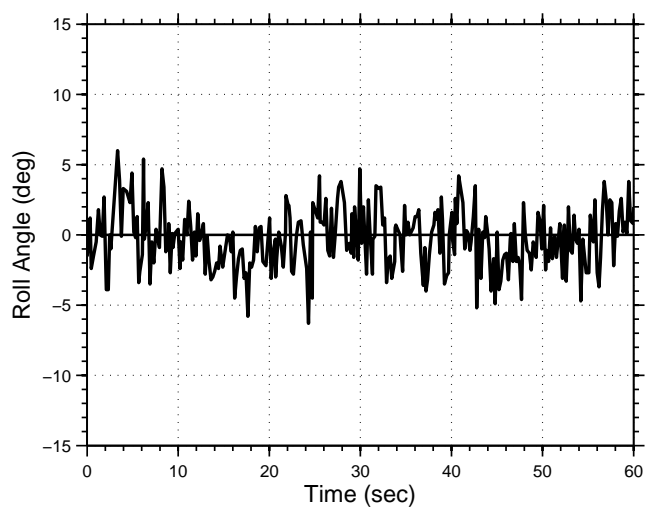

(d)

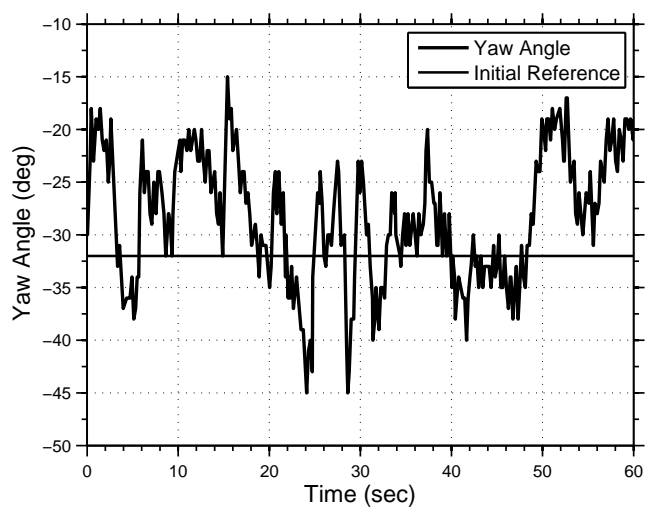

(f)

Figure 53: PID outdoor position control plots. (a) X-axis; (b) Pitch angle; (c) Y-axis; (d) Roll angle; (e) Z-axis; and (f) Yaw angle. 


\section{Chapter 6}

\section{Hybrid Control}

Evaluation of the simulation results point towards the adoption of a hybrid controller as a preferred technique for the quadrotor in an urban wind scenario. This chapter details the proposed hybrid scheme and again evaluates simulation test cases to assess the performance of the proposed hybrid control technique. Experimental flights with the proposed hybrid control implemented are also conducted to compare the simulation with the experimental test flight results.

\subsection{Proposed Hybrid Control Scheme}

Based on the results of the previous simulations, an in-depth focus on both the PID and AIB controllers is needed. Consider the point-B simulation experiments for the passing vortex test case. The $\mathrm{X}$-axis performance results for the PD and AIB controllers are shown in Figure 43(a). The problematic nature of the PD response, with the quadrotor's position barely approaching within $2 \mathrm{~m}$ of the desired hold point, is highlighted by the maximum error of $5.99 \mathrm{~m}$ and AOAD of $4.18 \mathrm{~m}$, which are the worst values recorded for this case of all the assessed control methods. The AIB

response, on the other hand, oscillates about the desired location with an AOAD of $1.47 \mathrm{~m}$, but also has a significant maximum error of $4.12 \mathrm{~m}$. 
In order to interpret why this particular case presents a challenge for the PD control, the nature of the wind disturbance needs to be examined. A histogram of the velocity of the LES simulation wind present at point-B on the $\mathrm{X}$-axis is shown in Figure 54. The histogram format serves to illustrate the distribution of the wind disturbance that TARA would experience if it were to achieve the desired $60 \mathrm{~s}$ hover flight within a body length of point-B. As depicted, the wind velocity ranges between -2.18 and $5.68 \mathrm{~m} / \mathrm{s}$, indicating reversals of flow, with a mean value of $2.53 \mathrm{~m} / \mathrm{s}$. The standard deviation of $1.55 \mathrm{~m} / \mathrm{s}$ is the highest on any axis of the six test points. An asymmetry is also evident in the data, as the distribution displays a negative skew with an elongated left tail.

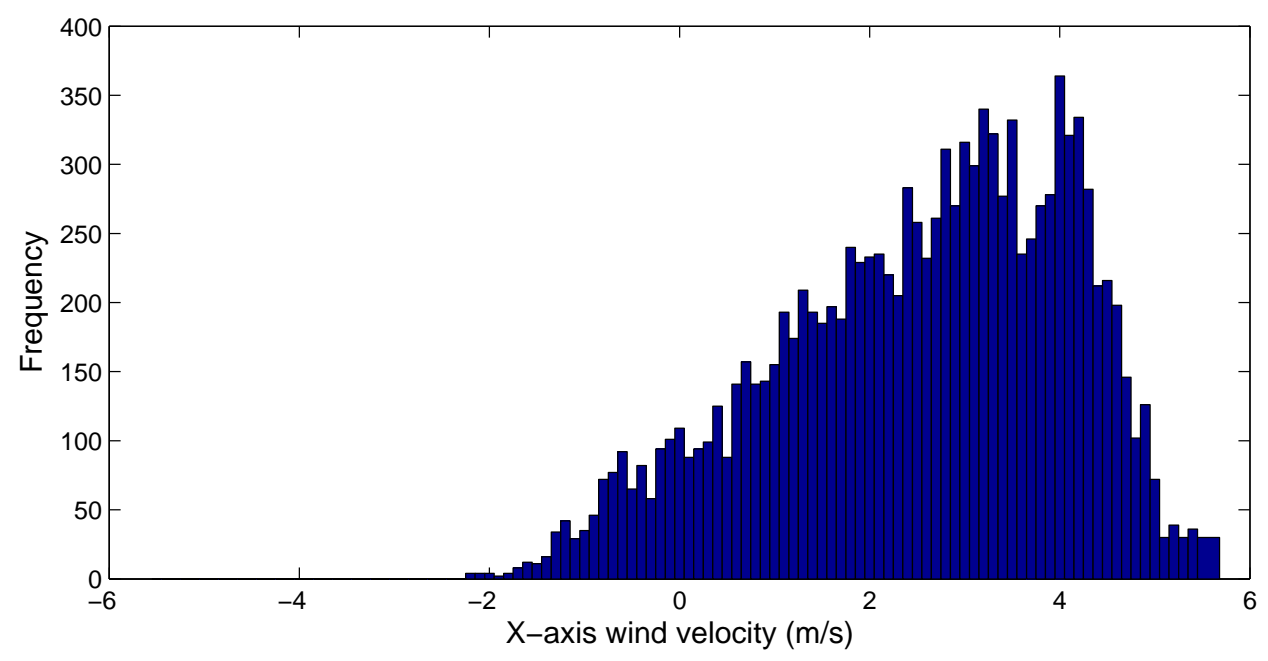

Figure 54: Distribution of $\mathrm{X}$-axis wind at point-B.

The $U_{X}$ control outputs generated by the PD and AIB controllers in response to this passing vortex scenario are substantially different. The PD X-axis control output, depicted in Figure 55(a), highlights the fact that the preponderance of the control effort is weighted towards the extremes of the distribution, representing maximal control output. The AIB controller requires less control effort in response to the wind disturbance, as illustrated in Figure 55(b). Similar results are also obtained for the 
$\mathrm{PD}$ and AIB controllers at point-D on the $\mathrm{X}$-axis, where the wind distribution has a mean velocity of $3.11 \mathrm{~m} / \mathrm{s}$ and also displays a substantial negative skew.

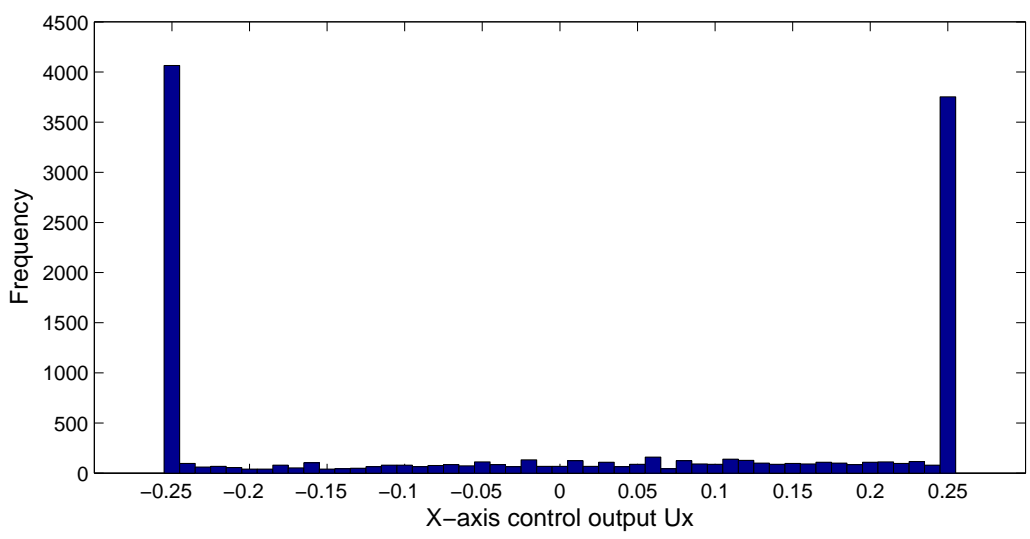

(a) $\mathrm{PD}$

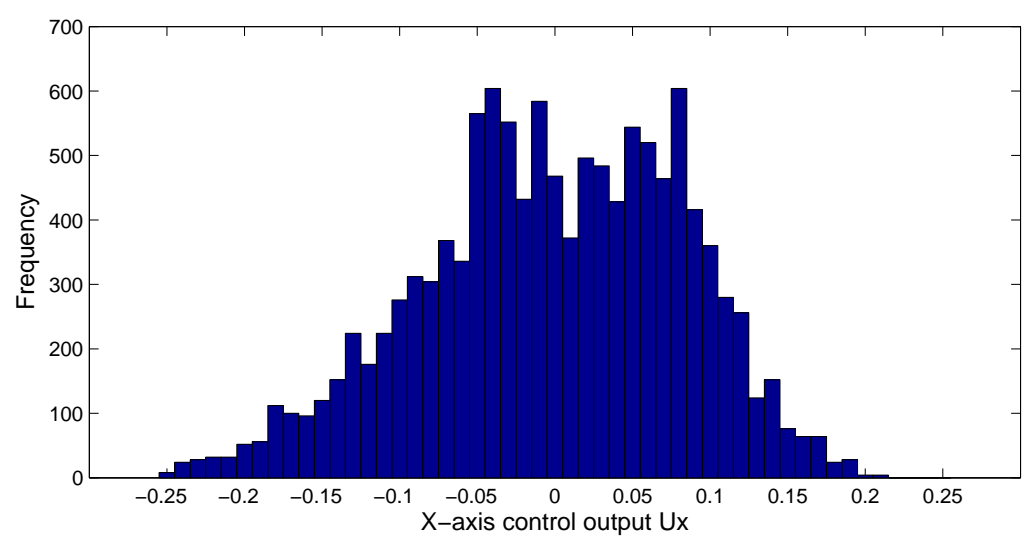

(b) AIB

Figure 55: X-axis control output for position hold at point-B.

By comparison, the $\mathrm{X}$-axis simulation results for the accelerated freestream wind at point-E are presented in Figure 46(a) for the PD and AIB controllers. For this test case, in marked contrast to the point-B results, the PD controller produces the best maximum error and AOAD results of any of the four control techniques tested, with excellent values of $0.33 \mathrm{~m}$ and $0.09 \mathrm{~m}$, respectively. Ranking the controllers with regards to performance metrics, the AIB controller's maximum error $(1.20 \mathrm{~m})$ and $\operatorname{AOAD}(0.48 \mathrm{~m})$ values are second best. The histogram in Figure 56 reveals the 
distribution of the wind present at point-E on the X-axis over the 60 s hover period. A clear distinction is evident between the attributes of the $\mathrm{X}$-axis wind disturbance at point-E compared with point-B. While the $4.53 \mathrm{~m} / \mathrm{s}$ mean wind velocity at point-E is actually higher than for point-B, a much smaller $0.25 \mathrm{~m} / \mathrm{s}$ standard deviation is found, with negligible skew demonstrated.

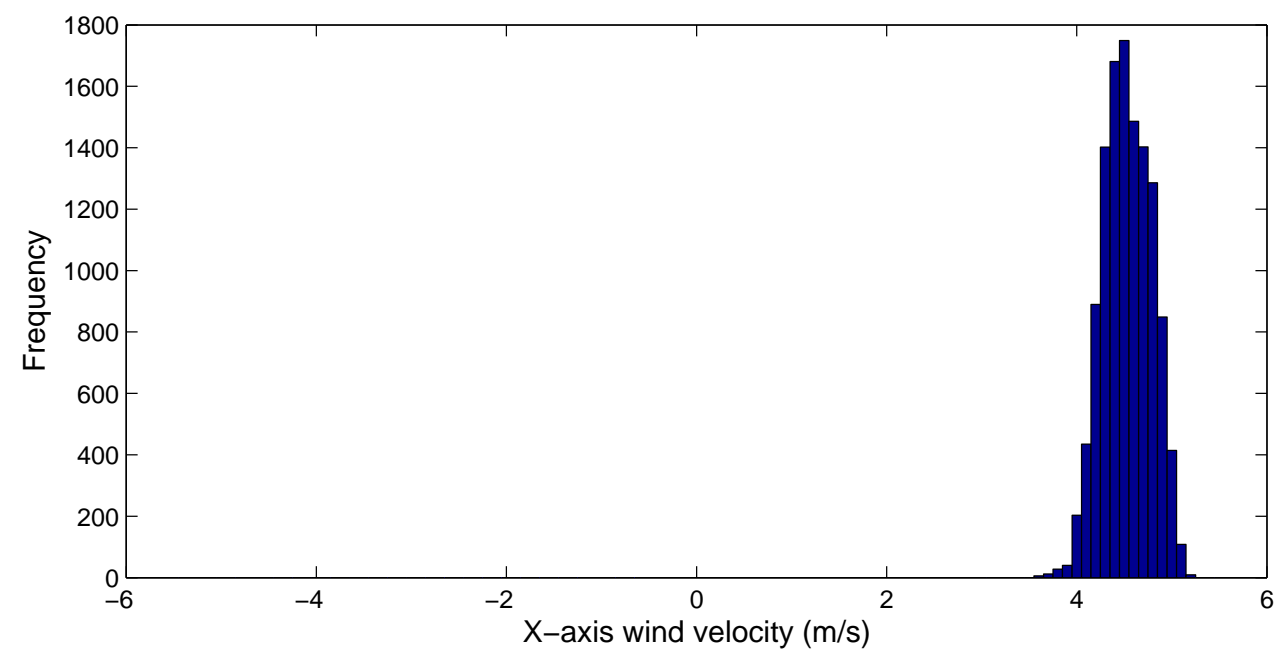

Figure 56: Distribution of $\mathrm{X}$-axis wind at point-E.

The $U_{X}$ control outputs generated for the accelerated freestream simulation for the PD and AIB controllers are shown in Figures 57(a) and 57(b), respectively. The PD control effort is much more effectively distributed across the available range than it is for the passing vortex simulation. Similar results for the freestream wind simulation at point-F confirm the ability of the PD controller in dealing with sustained disturbances of this nature. The AIB output demonstrates a minimal control effort while producing an "acceptable" control performance.

Focusing on the PD and AIB control performances for these two test cases underscores certain salient points to consider when achieving the stated goal of selecting an appropriate control technique for the urban wind environment. First, the PD design produces a substantially different control outcome based on the characteristics 


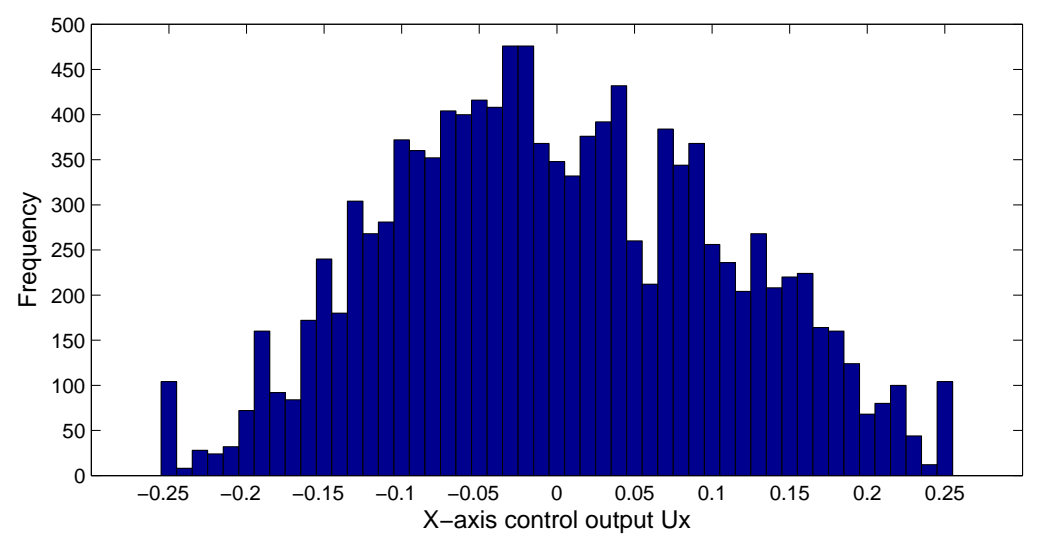

(a) PD

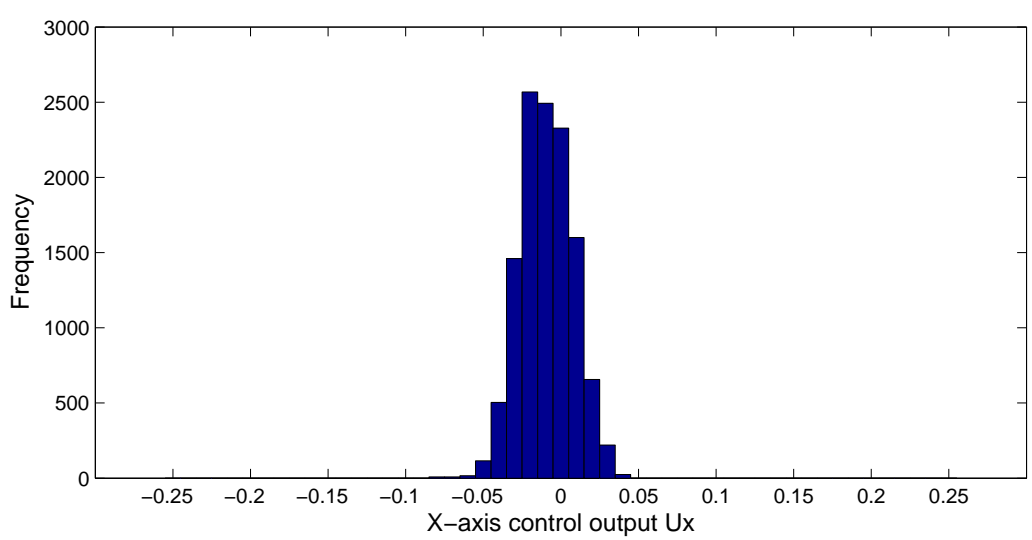

(b) AIB

Figure 57: X-axis control output for position hold at point-E.

of the wind distribution. Furthermore, no one control technique is able to demonstrate a "good" performance grade, as defined in Table 2, throughout all of these wind disturbance test cases.

The PD and AIB controllers each produce good results in differing scenarios and display complementary strengths. The AIB controller is able to consistently bring the quadrotor back to a desired hold location, bounding its motion, regardless of the disturbance present. AIB also has the advantage of being an adaptive control, as well as fulfilling Lyapunov stability, which are desirable characteristics when dealing with perturbations in the form of varying wind conditions. On the other hand, the PD 
control proves to be good at holding a position in non-turbulent freestream conditions, as well as avoiding errors, forcing the tracking error towards zero.

Therefore, a hybrid control scheme which uses a PD/PID control to hold the quadrotor's position once it is within \pm 1 m of a target position and an AIB controller at all other times is proposed and tested. The $\pm 1 \mathrm{~m}$ threshold is selected based on the "good" (error $\leq 1 \mathrm{~m})$ limit defined in the performance metric.

\subsection{Hybrid Position Control Simulations}

The proposed hybrid control design is tested in the presence of wind for the six defined positions around the single building geometry as per the simulation methodology set out in Section 3.3. Table 20 summarizes the maximum error and AOAD values obtained for the hybrid controller for each of the points A through $\mathrm{F}$. The final column lists the highest maximum error and average AOAD results of the hybrid simulations on each axis.

Table 20: In wind hybrid control performance summary.

\begin{tabular}{|c|c|c|c|c|c|c|c|c|}
\hline & \multicolumn{6}{|c|}{ Point } & \multirow[b]{2}{*}{ Max/Avg } \\
\hline & & A & $\mathrm{B}$ & $\mathrm{C}$ & $\mathrm{D}$ & $\mathrm{E}$ & $\mathrm{F}$ & \\
\hline \multirow{2}{*}{$\mathrm{X}$-axis } & Max. Error $(\mathrm{m})$ & 0.84 & 3.57 & 2.20 & 2.73 & 0.82 & 1.08 & 3.57 \\
\hline & $\operatorname{AOAD}(\mathrm{m})$ & 0.32 & 1.35 & 0.66 & 0.81 & 0.25 & 0.24 & 0.61 \\
\hline \multirow{2}{*}{ Y-axis } & Max. Error $(\mathrm{m})$ & 2.13 & 2.47 & 2.18 & 1.75 & 1.83 & 0.98 & 2.47 \\
\hline & $\mathrm{AOAD}(\mathrm{m})$ & 0.69 & 0.83 & 0.82 & 0.37 & 0.53 & 0.24 & 0.58 \\
\hline \multirow{2}{*}{ Z-axis } & Max. Error $(\mathrm{m})$ & 0.86 & 1.13 & 1.83 & 0.30 & 0.37 & 0.18 & 1.83 \\
\hline & $\mathrm{AOAD}(\mathrm{m})$ & 0.27 & 0.27 & 0.54 & 0.08 & 0.10 & 0.04 & 0.22 \\
\hline
\end{tabular}




\subsubsection{Dead Air Zone (Point-A)}

Figure 58 depicts the hybrid controller performance results for hover flight at point-A. For comparison purposes, the corresponding PD/PID and AIB simulation outcomes, as originally presented in Chapter 5, are also plotted throughout for each axis.

The results for the hybrid controller demonstrate adequate position hold in the chaotic gusts of the dead air zone. As listed in Table 20, the hybrid control produces a maximum error of $0.84 \mathrm{~m}$ on the $\mathrm{X}$-axis, improving upon the values obtained by both the PD and AIB controllers. A "good" AOAD of $0.32 \mathrm{~m}$ is also achieved, bettering the performance of the AIB controller and comparable to the PD results, as can be seen in Figure 58(a). Similar comparative results are depicted in Figure 58(b) for the Y-axis where the wind fluctuations are greater than along the X-axis (see Figure 16(a) and 16(b)). For the Z-axis, the hybrid control produces slightly better maximum error $(0.86 \mathrm{~m})$ and AOAD $(0.26 \mathrm{~m})$ values than either the PID or AIB controllers, although as Figure 58(c) demonstrates, this difference is not significant.

\subsubsection{Passing Vortex (Point-B)}

As previously detailed in Section 6.1, the passing vortex case provides a considerable challenge for the PD controller, generating a "bad" performance (error $>5 \mathrm{~m}$, as characterized by the defined metric in Table 2). While the AIB outcome is consistently better than PD for the duration of the point-B simulation, large positional errors are also evidenced. The X-axis results, shown in Figure 59(a), establish the hybrid control's enhanced ability in dealing with the high mean, skewed wind field present in this test case. The maximum error and AOAD values for the hybrid controller, at $3.57 \mathrm{~m}$ and $1.35 \mathrm{~m}$, respectively, are better than those obtained by both the PD and AIB controls, demonstrating an improvement in directing the quadrotor's position about the desired reference. However, these values also represent the 


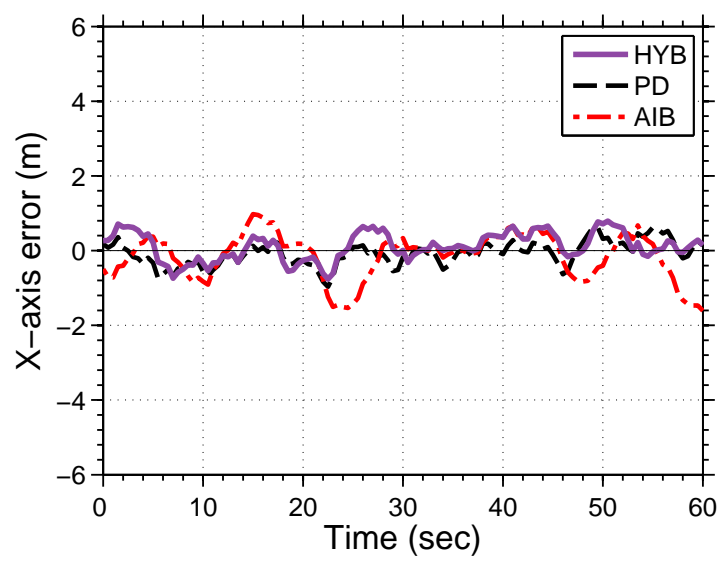

(a)

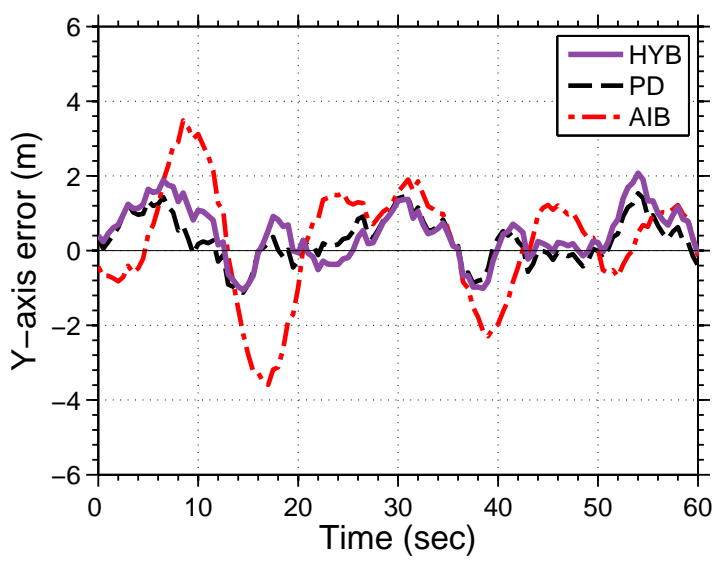

(b)

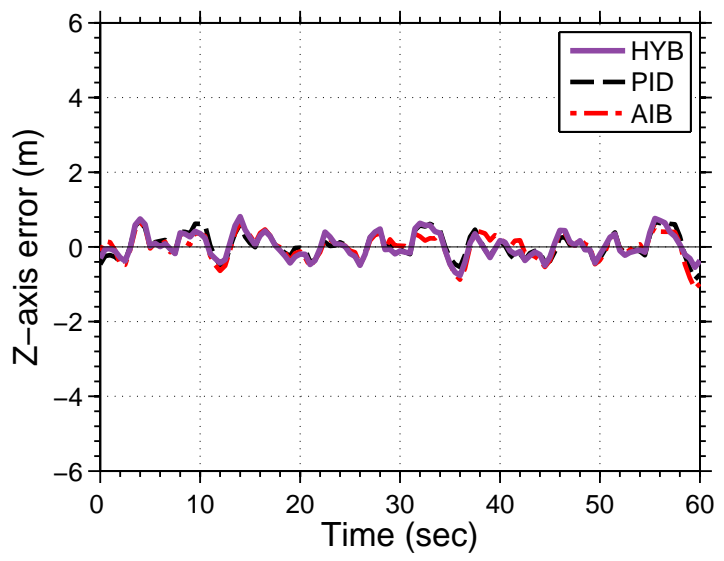

(c)

Figure 58: In wind hybrid position hold for dead air zone (point-A). (a) X-axis; (b) Y-axis; and (c) Z-axis.

overall worst performance results achieved by the hybrid control. Of all of the six test cases considered, the AOAD achieved here is the only value to fall beyond the "good" performance metric, to be classified as "acceptable". Similarly, of all of the test cases, this is the sole maximum error result to be classified as worse than an "acceptable" performance, rating as "poor".

The $\mathrm{Y}$-axis gusts demonstrate a similar asymmetry and range as the $\mathrm{X}$-axis; however, with a lower mean velocity. Again, as shown in Figure 59(b), the hybrid control displays an advantage under these biased conditions overcoming the inability of the 
PD controller to bring the quadrotor's position to the desired location as well as reducing the errors exhibited by the AIB control (while keeping TARA's motion centered about the designated setpoint). The maximum error $(2.47 \mathrm{~m})$ and AOAD values obtained along the $\mathrm{Y}$-axis at point-B are also the worst values obtained across all points A through F considered.

For the Z-axis, presented in Figure 59(c), the hybrid controller produces a slightly higher maximum error and AOAD than either the PID or AIB controls, but again, the difference between all three controllers is negligible.

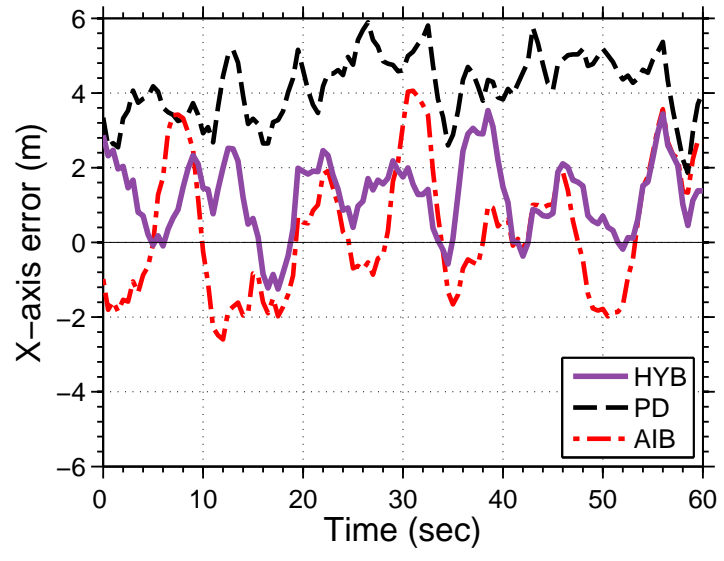

(a)

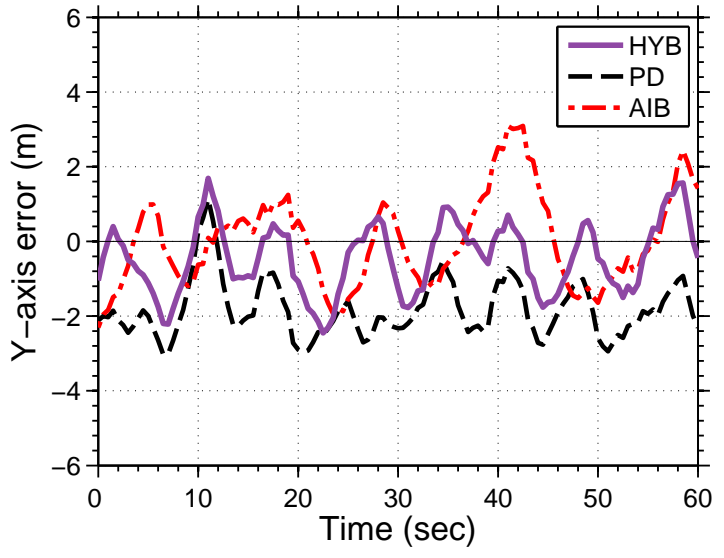

(b)

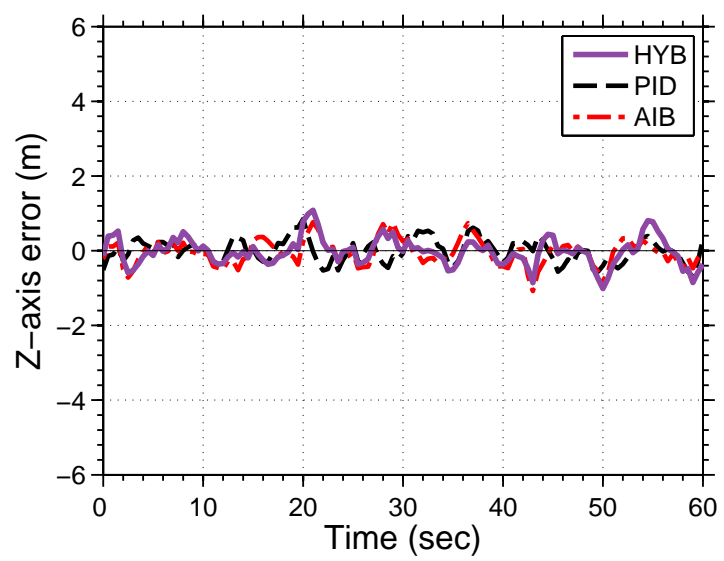

(c)

Figure 59: In wind position hold for passing vortex (Point-B). (a) X-axis; (b) Y-axis; and (c) Z-axis. 


\subsubsection{Wake Eddy (Point-C)}

The position control results for the hybrid controller at point-C are shown in Figure 60. For the X-axis, the hybrid control closely follows the PD outcome, producing a slightly lower AOAD of $0.66 \mathrm{~m}$, with an increased maximum error of $2.20 \mathrm{~m}$. Similarly, the Y-axis hybrid control results for the highest ranging winds tested are closely related to the $\mathrm{PD}$, with an increased maximum error. The AOAD for this axis represents the only case where the AOAD recorded by the hybrid controller $(0.82 \mathrm{~m})$ is marginally worse than both the PD and AIB performance. Like the other control techniques considered, the hybrid control has its worst altitude hold for the wake eddy case (which has the highest standard deviation in velocity for this axis of $1.17 \mathrm{~m} / \mathrm{s}$ ). However, comparing values in Tables 20 and 14 the hybrid control yields comparable values in both maximum error and AOAD with the "acceptable" $(1<$ error $\leq 3 \mathrm{~m})$ and "good" performance (error $\leq 1 \mathrm{~m}$ ) levels respectively.

\subsubsection{Downwash (Point-D)}

The $\mathrm{X}$-axis wind in the downwash is the most highly skewed of all the assessed position hold regions. As depicted in Figure 61(a), the hybrid controller is able to ameliorate the performance of both the PD and AIB controls, bringing the quadrotor's position towards the desired coordinates better than the PD control, and maintaining a better hold than the AIB control. The improved maximum error and AOAD values of 2.73 $\mathrm{m}$ and $0.81 \mathrm{~m}$, respectively, reflect this fact. When considered in conjunction with the simulation results for point-B, the hybrid controller demonstrates a clear advantage in turbulent conditions characterized by high mean velocity skewed winds.

For the pronounced gusts of the Y-axis, a "good" AOAD of $0.37 \mathrm{~m}$ is obtained for the Y-axis by the hybrid controller. As can be seen in Figure 61(b), the hybrid performance falls in between that of the PD and AIB controls. 


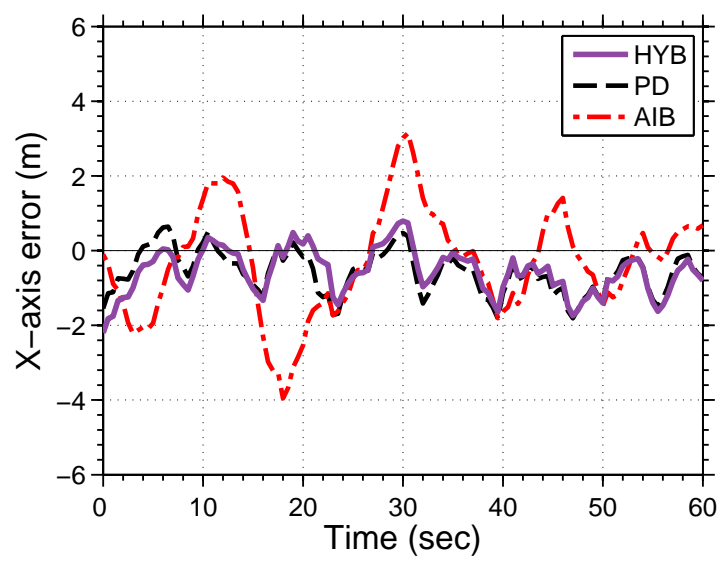

(a)

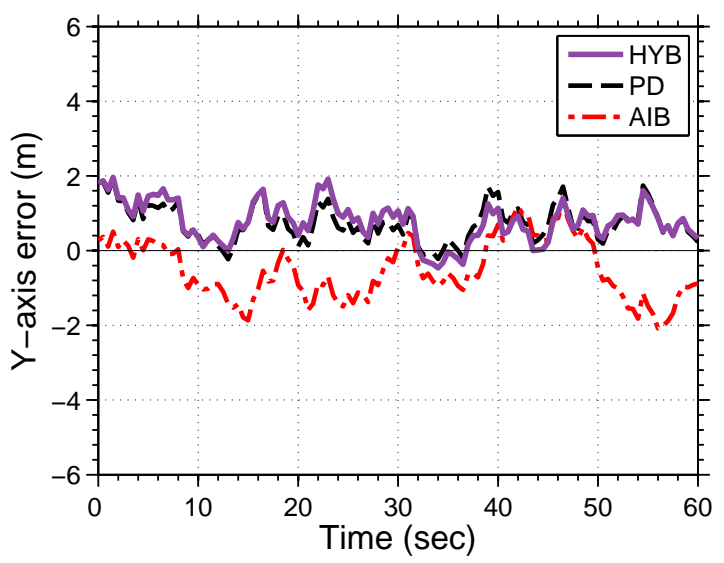

(b)

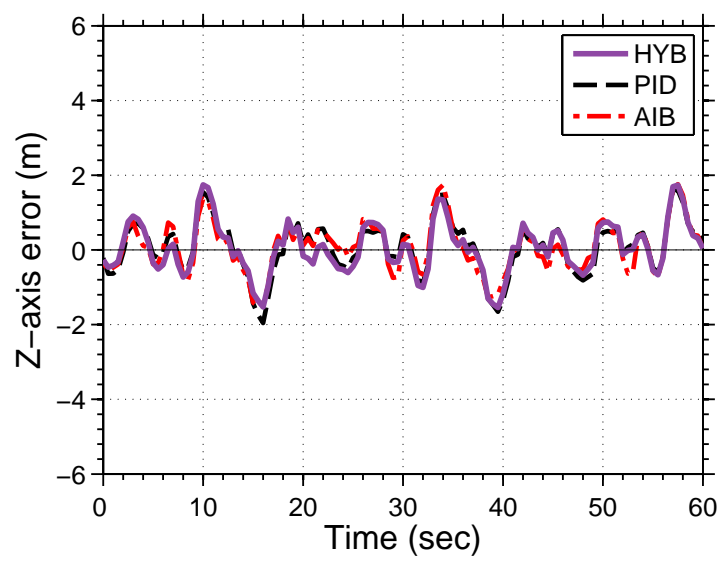

(c)

Figure 60: In wind position hold for wake eddy (point-C). (a) X-axis; (b) Y-axis; and (c) Z-axis.

Despite the presence of the greatest mean velocity wind on the Z-axis at point-D, a $0.08 \mathrm{~m}$ AOAD is achieved using the hybrid controller as shown in Figure 61(c).

\subsubsection{Accelerated Freestream (Point-E)}

For the sustained $4.51 \mathrm{~m} / \mathrm{s}$ wind on the X-axis of the accelerated freestream, the hybrid control produces "good" maximum error and AOAD values of $0.82 \mathrm{~m}$ and $0.25 \mathrm{~m}$, respectively. For the Y-axis, an "acceptable" maximum error $(1.83 \mathrm{~m})$ and "good" AOAD $(0.53 \mathrm{~m})$ are produced. Figures 62(a) and 62(b) illustrate that the 


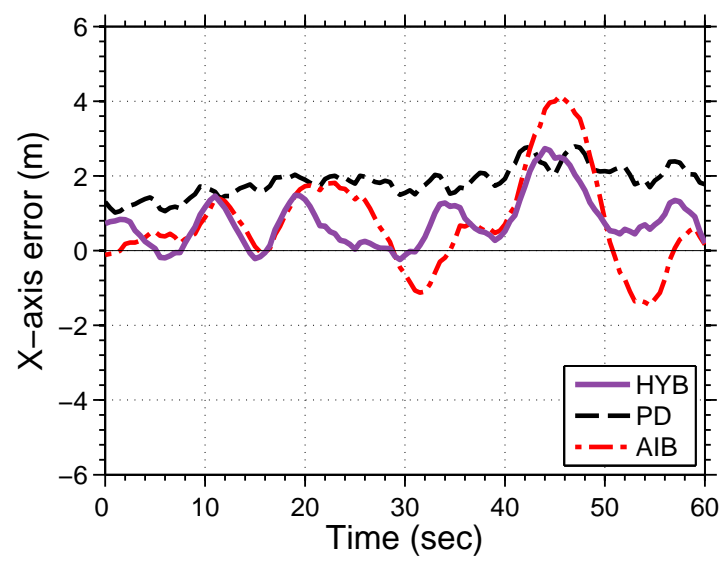

(a)

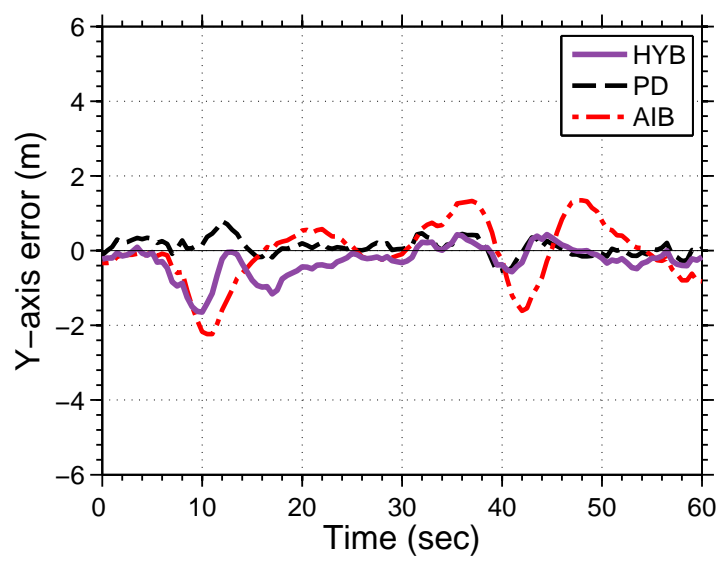

(b)

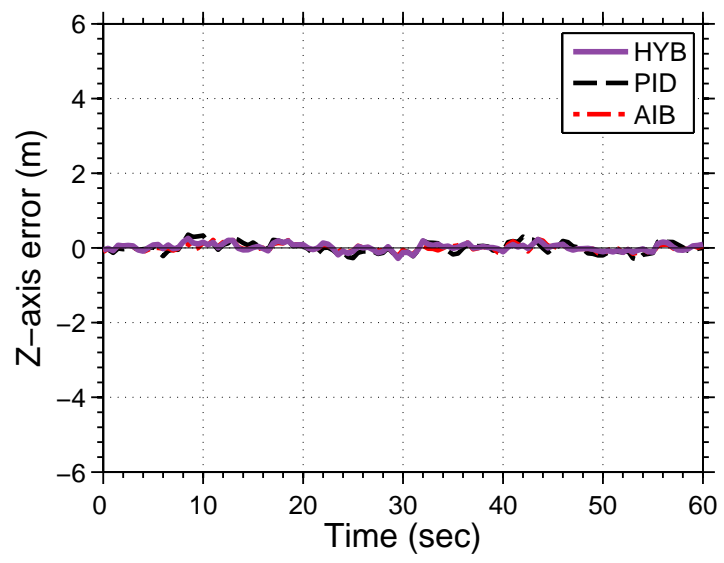

(c)

Figure 61: In wind position hold for downwash (point-D). (a) X-axis; (b) Y-axis; and (c) Z-axis.

larger positional errors occur predominantly to one side of the desired hold value, for both the $\mathrm{X}$-axis and $\mathrm{Y}$-axes. For the $\mathrm{Y}$-axis, significant improvements are displayed over the AIB simulation, while not quite as good as the PD performance. The Z-axis evidences "good" maximum error $(0.37 \mathrm{~m})$ and AOAD $(0.10 \mathrm{~m})$ values, with little appreciable difference between the assessed controls. 


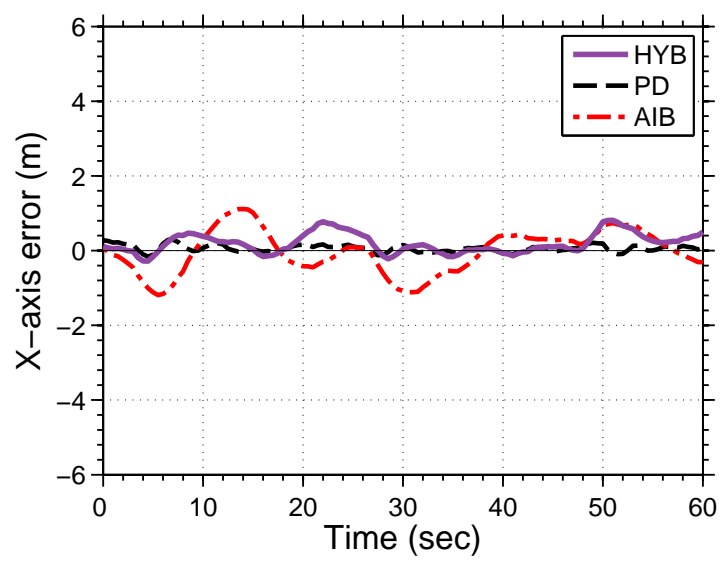

(a)

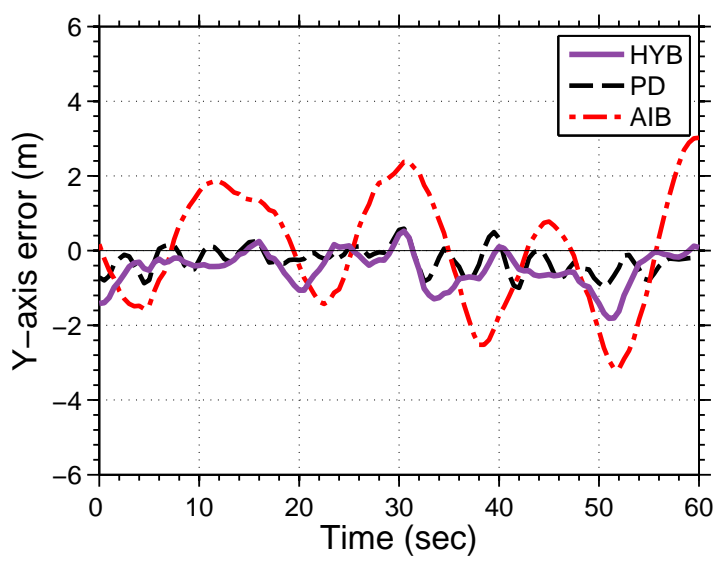

(b)

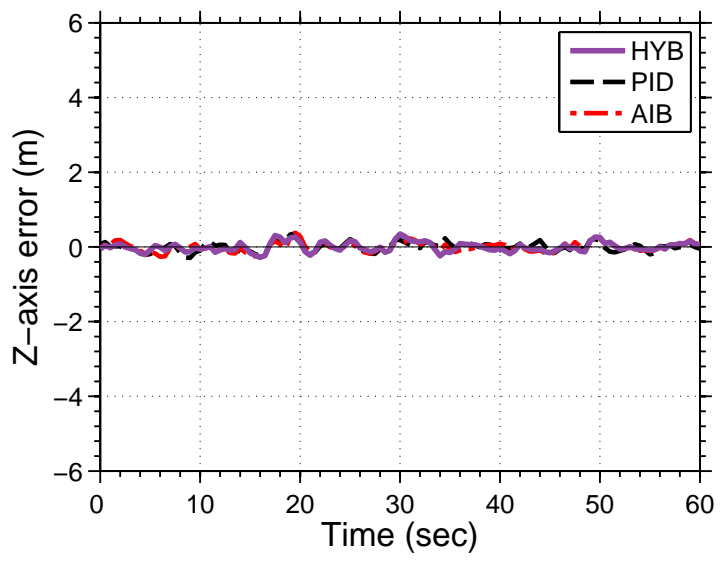

(c)

Figure 62: In wind position hold for accelerated freestream (point-E). (a) X-axis; (b) Y-axis; and (c) Z-axis.

\subsubsection{Freestream Wind (Point-F)}

The performance the freestream wind test scenario, illustrated in Figure 63, provides some of the best results for the hybrid controller. The X-axis AOAD of $0.24 \mathrm{~m}$ is the lowest obtained for this axis. Furthermore, the maximum error $(0.98 \mathrm{~m})$ and AOAD $(0.24 \mathrm{~m})$ values represent the best results for the $\mathrm{Y}$-axis, and are better than those obtained in both the PD and AIB simulations. The best overall maximum error $(0.18$ $\mathrm{m})$ and $\mathrm{AOAD}(0.04 \mathrm{~cm})$ for the Z-axis also occur at point-F. 


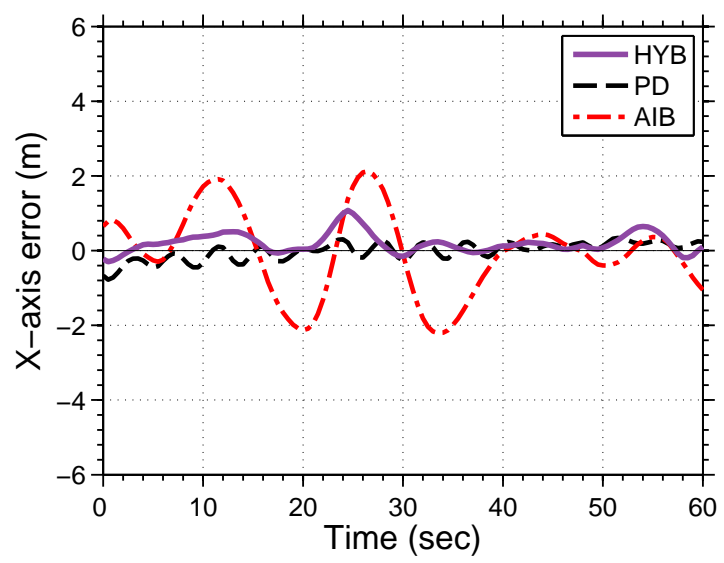

(a)

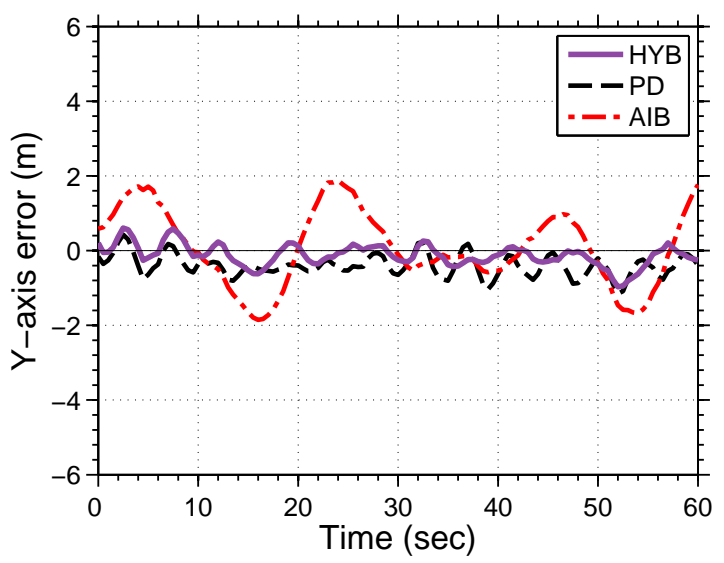

(b)

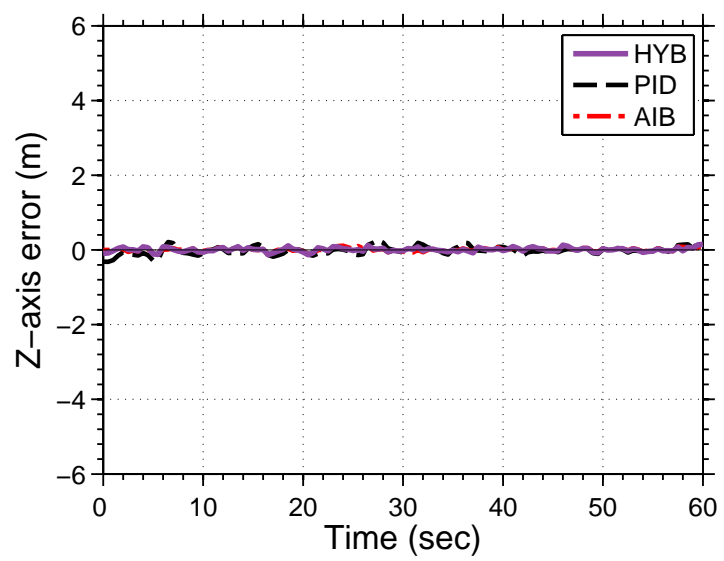

(c)

Figure 63: In wind position hold for freestream wind (point-F). (a) X-axis; (b) Y-axis; and (c) Z-axis.

\subsection{Summary of Hybrid Position Control Simula- tions}

The simulation results of the hybrid control demonstrate the intended performance improvements motivating its design. At point-B and point-D on the $\mathrm{X}$-axis, highmean asymmetrical gusts challenge the PD control, resulting in AOAD values of 4.18 $\mathrm{m}$ and $1.85 \mathrm{~m}$, respectively. The hybrid control improves upon the PD position hold results in these turbulent conditions, with respective AOADs of $1.35 \mathrm{~m}$ and $0.81 \mathrm{~m}$. 
Both the AOAD and maximum error obtained by the hybrid controller at these points are better than the corresponding values demonstrated by the PD, as well as the AIB controller. On the other hand, the freestream conditions present at point-F on the X-axis yield "good" maximum error $(0.78 \mathrm{~m})$ and AOAD $(0.19 \mathrm{~m})$ values for the PD control, with the AIB controller comparatively struggling to hold position with a higher maximum error $(2.21 \mathrm{~m})$ and $\operatorname{AOAD}(0.87 \mathrm{~m})$. Here the hybrid controller improves upon the AIB values with maximum error $(1.08 \mathrm{~m})$ and $\operatorname{AOAD}(0.24 \mathrm{~m})$ results comparable to the PD performance. Similar results are also obtained for the accelerated freestream at point-E.

Examining the performance results over all of the test cases for all three axes, the highest maximum error produced by the hybrid controller is $3.57 \mathrm{~m}$, compared with $5.99 \mathrm{~m}$ for the PID controller, representing a $40 \%$ improvement, and $4.17 \mathrm{~m}$ for the AIB controller, corresponding to a $14 \%$ improvement. Similarly, over the six simulation points investigated, the hybrid controller has an average AOAD of 0.47 $\mathrm{m}$, while the PID and AIB control have similar respective average AOADs of $0.71 \mathrm{~m}$ and $0.70 \mathrm{~m}$, for $34 \%$ and $33 \%$ improvements. Figure 64 presents the average AOAD and highest maximum error obtained for the X, Y, and Z-axes for the PID, AIB and hybrid controllers, visually depicting the hybrid control's performance improvement over the former two techniques. On average, the hybrid controller maintains the quadrotor's location within a body length of the target hold position.

\subsection{Experimental Outdoor Flight using Hybrid Position Control}

An outdoor experimental flight of the hybrid controller is conducted in wind gust conditions using the same setup as depicted in Figure 52 for the PID controller. 


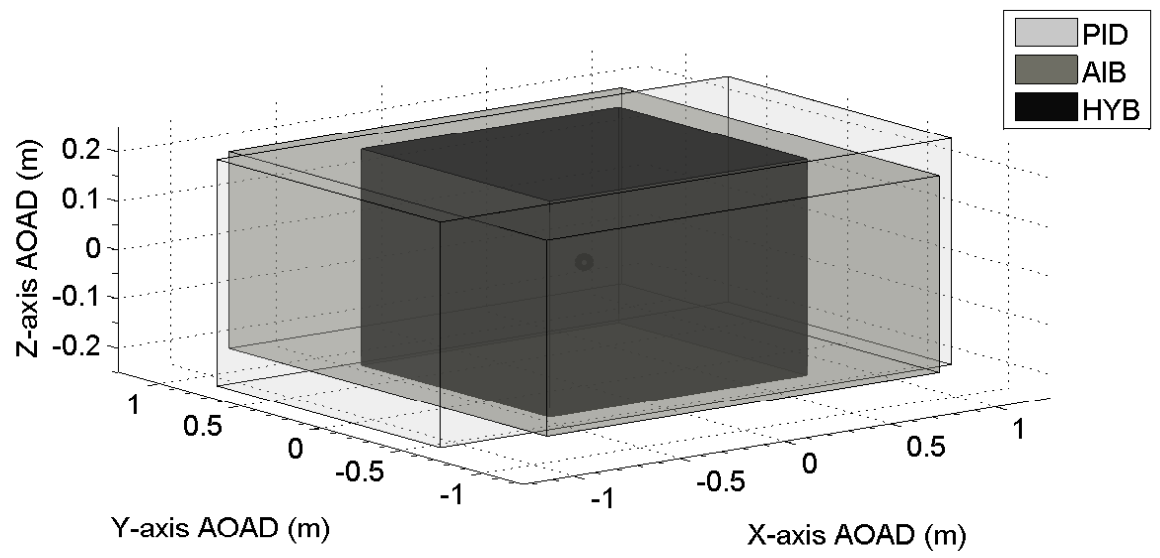

(a) Average AOAD

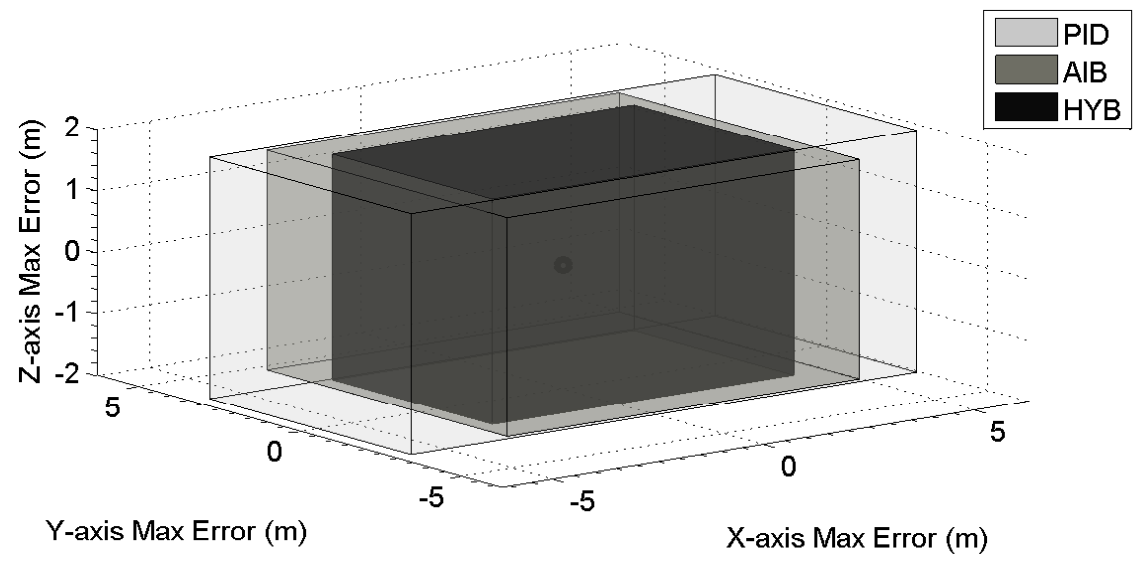

(b) Highest maximum error

Figure 64: PD/PID, AIB and hybrid controller error comparison

Average wind speeds of $4 \mathrm{~m} / \mathrm{s}$ (Beaufort scale value $=3$ ) with gusts up to $7 \mathrm{~m} / \mathrm{s}$ were indicated by the weather forecast. The weather station placed on the roof of the stairwell recorded winds of $1.4 \mathrm{~m} / \mathrm{s}$ on average. Flight conditions correspond to a Reynold's number of $R_{e}=5.6 \times 10^{5}$, compared with the simulation Reynold's number of $R_{e}=7.3 \times 10^{6}$. During the flight experiment several significant gusts ranging up to $12 \mathrm{~m} / \mathrm{s}$ were observed. From the flight video, strong gusts are evident at time marks of $9,22,31,40,44,52$ and 58 seconds. Figures $65(\mathrm{a})$ and $65(\mathrm{c})$ indicate the wind direction at 5 second intervals throughout the flight, based on the weather vane orientation of the weather station. 
Table 21 summarizes the performance results for the outdoor hybrid controller flight. Despite the presence of larger wind disturbances than for the PID outdoor test, the hybrid control is able to maintain a "good" AOAD for the X, Y, and Z-axes, with values of $0.83 \mathrm{~m}, 0.59 \mathrm{~m}$, and $0.19 \mathrm{~m}$, respectively. As shown by the dashed lines in Figure 65(a), the sonar data for the X-axis produces spurious spikes (refer to Appendix-B for details on sonar glitches) at 24 and 46 seconds into the flight. Neglecting these sonar artifacts, the maximum error for the $\mathrm{X}$-axis is evaluated at an "acceptable" $2.12 \mathrm{~m}$. The controller exhibits the responsiveness for which it is designed, rapidly and consistently counteracting gust disturbances to regulate the quadrotor's position towards the desired location. The Y-axis data also evidences a sonar artifact at 49 seconds, as indicated in Figure 65(c), without which a $1.44 \mathrm{~m}$ maximum error is measured. Both the roll and pitch angles evidence "good" AOADs, with values of 1.48 and 2.29 degrees, respectively, along with "acceptable" rated maximum errors. The effect of electromagnetic interference is reflected in greater yaw variability, with an "acceptable" AOAD and "poor" maximum error.

The experimental hybrid controller results are consistent with the highest maximum error and average AOAD values for the six simulated hybrid test cases, summarized in Table 20. For the respective X, Y, and Z axes differences of $0.19 \mathrm{~m}, 0.00$ $\mathrm{m}$, and $0.03 \mathrm{~m}$ exist between the simulation average AOADs and the experimental AOAD values. The experimental maximum error results are bounded by the simulation highest maximum error values of $3.57 \mathrm{~m}, 2.47 \mathrm{~m}$, and $1.83 \mathrm{~m}$ for the $\mathrm{X}, \mathrm{Y}$, and $\mathrm{Z}$ axes respectively. In general, the experimental position control performance values are consistent with the simulation data.

The stochastic nature of wind makes it impossible to recreate identical airflow conditions in an outdoor environment. Although the PD/PID and hybrid controllers face different wind velocities and turbulent effects in their outdoor tests, some generalized 
observations can be appropriately drawn. On the whole, the outdoor flight experiments effectively validate the expected flight control characteristics predicted by the simulator. When close to the desired hold position, the PID control demonstrates a better ability to maintain the setpoint, with the hybrid control producing a comparable, but slightly higher AOAD. However, when gusts cause the quadrotor's position to deviate, the hybrid controller quickly and reliably converges TARA's position back towards the desired coordinates, in comparison with the PID controller. Although the hybrid controller is tested under larger magnitude gusts, unlike the PD/PID controller, it is able to maintain a "good" AOAD and "acceptable" maximum error on all 3 axes, when accounting for sonar noise. Comparing the experimental outdoor PD/PID results in Table 18 with the corresponding hybrid results in Table 21, similar maximum errors and AOADs are observed for the angles. The X, Y, and Z-axes position maximum errors are also similar between the PD/PID and hybrid controllers. The hybrid controller improves the X-axis and Z-axis AOAD results obtained by the PD/PID control by $34 \%$ and $40 \%$, respectively, while the Y-axis AOAD is comparatively worse by $19 \%$.

Table 21: Hybrid outdoor position control summary.

\begin{tabular}{|l|c|c|c||c|c|c|}
\cline { 2 - 7 } \multicolumn{1}{c|}{} & \multicolumn{3}{c||}{ Angles (deg) } & \multicolumn{3}{c|}{ Position (m) } \\
\cline { 2 - 7 } \multicolumn{1}{c|}{} & Roll & Pitch & Yaw & X-axis & Y-axis & Z-axis \\
\hline Max. Error & 7.00 & 10.00 & 14.00 & 2.12 & 1.46 & 0.48 \\
\hline AOAD & 1.48 & 2.29 & 7.27 & 0.80 & 0.58 & 0.19 \\
\hline
\end{tabular}




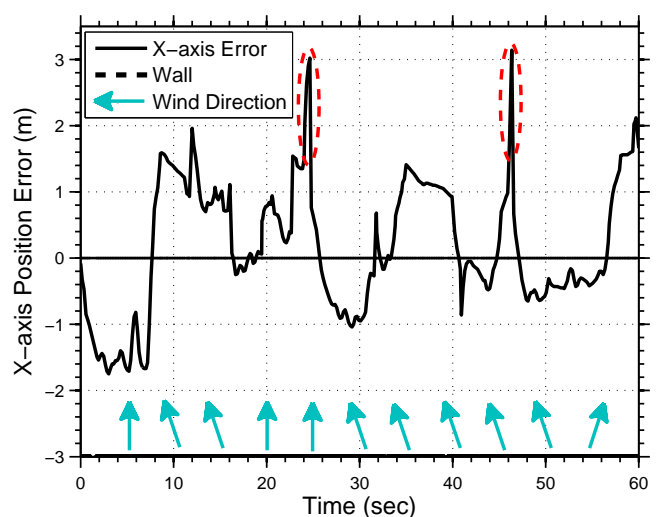

(a)

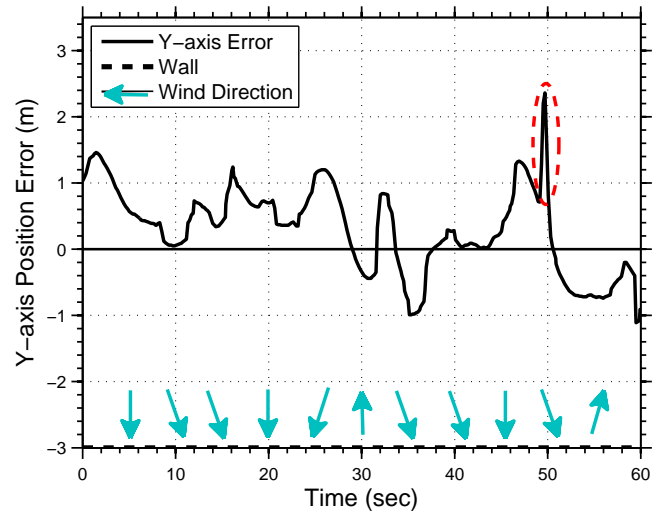

(c)

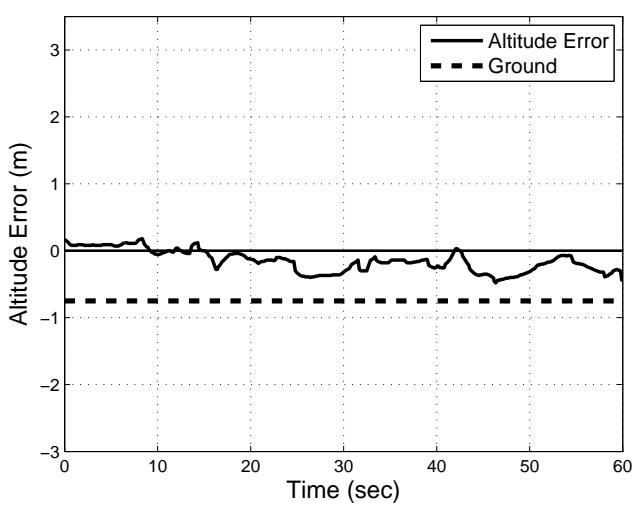

(e)

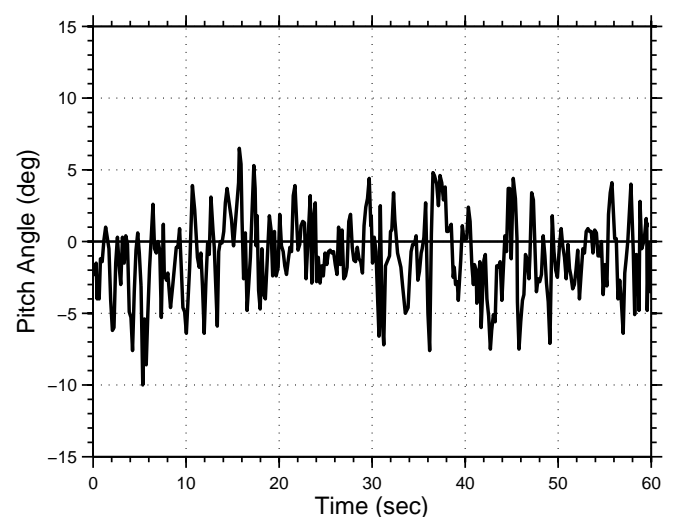

(b)

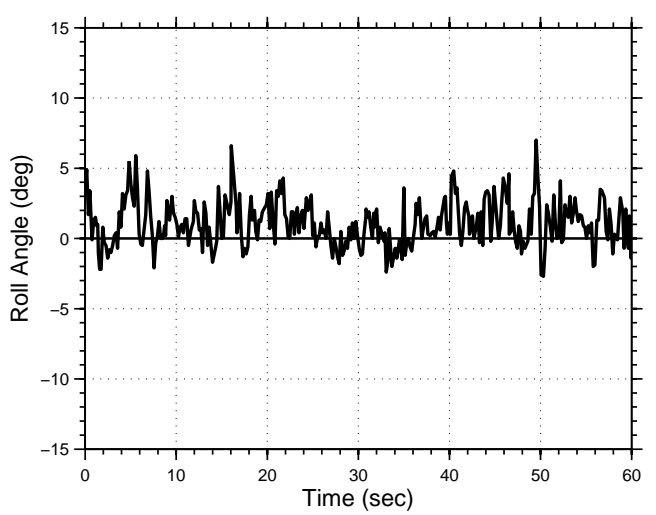

(d)

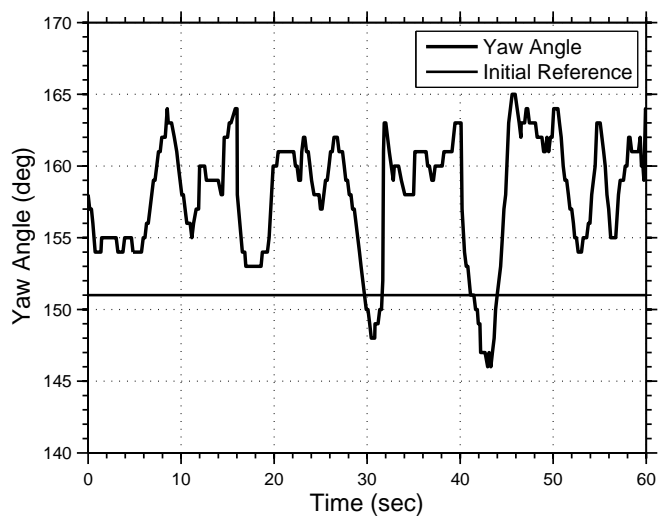

(f)

Figure 65: Hybrid outdoor position control plots. (a) X-axis; (b) Pitch angle; (c) Y-axis; (d) Roll angle; (e) Z-axis; and (f) Yaw angle. 


\section{Chapter 7}

\section{Conclusion}

This thesis provides a foundation for the work required for safe and successful deployment of small unmanned aerial vehicles, specifically quadrotors, in urban settings. A literature review covering quadrotor flight control techniques, unmanned aerial vehicle flight control in the presence of wind disturbances, and urban wind modelling using computational fluid dynamics techniques is presented, and may serve as a reference for further research within this area. Based on the surveyed studies, a need to investigate the performance of quadrotors within the urban wind conditions into which they are being introduced is established.

To effectively replicate the urban wind environment, a time-dependent, threedimensional large eddy simulation technique is employed in modelling the wind wake of a building. The transient wind data generated through this computational fluid dynamics analysis captures spatially and temporally varying features such as vortex shedding, wake eddies, downwash, and flow reversals. The simulator has a modular architecture such that the urban environment can be altered to represent different areas of interest. Multiple flight control techniques are tested in this simulated wind environment.

A 3D printed quadrotor prototype known as TARA is also developed to experimentally validate various control techniques. It incorporates two critical features for 
urban deployment, a position control with centimetre accuracy, and an inherent sense and avoid capability through the use of sonar sensing.

Based on flight simulations which hold the quadrotor at various positions within a building wake no single control technique of the four tested (proportional integral derivative, integral backstepping, fuzzy, and adaptive integral backstepping) conclusively outperformed the others throughout all the representative wind conditions considered. The fuzzy controller provided effective control in low disturbance regions, but is generally slow in counteracting winds with high mean velocities. The integral backstepping controller didn't always center the quadrotor's position about the desired location, particularly under freestream conditions. The adaptive integral backstepping controller unfailingly did so, at the cost of occasionally higher deviations. A proportional integral derivative controller overall demonstrated satisfactory position hold performance in freestream wind, but is unable to attain a desired setpoint in asymmetrical high mean gusts. With the control techniques producing varying results based on the characteristics of the wind distribution, identifying the LES-based transient wind flow around a single building and selecting a number of test locations reflecting a variety of representative turbulence conditions afforded an important analysis element.

The simulation results demonstrate a tradeoff between maximizing boundedness and minimizing errors. Due to the risks associated with operating in urban areas, an approach which assures the safest quadrotor position control performance under the full range of expected turbulence conditions is preferred. In order to overcome urban wind disturbances, a hybrid flight control technique is proposed combining the characteristics of both a proportional integral derivative and an adaptive integral backstepping controller. The hybrid controller demonstrates the required flexibility to deal successfully with a variety of wind conditions. Over the six simulation points investigated, only the hybrid controller is able to maintain an average of absolute 
difference within the "good" range (error $\leq 1 \mathrm{~m})$, with the single exception of an "acceptable" performance for the X-axis control in the passing vortex case. With an overall average AOAD across all three position axes of $0.47 \mathrm{~m}$ the hybrid controller on average maintains the quadrotor's location within a body length of the target hold position. This $0.47 \mathrm{~m}$ average AOAD represents a $33 \%$ reduction in average AOAD as compared with the AIB controller, a $34 \%$ reduction over PD/PID, a $36 \%$ reduction over IB, and a $37 \%$ reduction over the fuzzy controller. The maximum error is also maintained by the hybrid controller within the "good-to-acceptable" range (error $\leq 3 \mathrm{~m})$. This highest maximum error represents a decrease of $14 \%$ over AIB, $28 \%$ compared with the fuzzy, $31 \%$ less than the IB, and a $40 \%$ reduction in the largest maximum error over the PD/PID controller.

Outdoor experiments confirm the design characteristics demonstrated by the PD/PID and hybrid controller simulation results. The highest maximum error values observed over the course of the simulations serve as a bound for the PD/PID and hybrid experimental results. "Good-to-acceptable" (error $\leq 3 \mathrm{~m}$ ) AOAD and maximum error values are maintained by the hybrid controller under wind gust conditions. Furthermore, the hybrid control exhibits both effective hold performance and the ability to quickly counteract sharp wind gust disturbances (up to $12 \mathrm{~m} / \mathrm{s}$ ). Overall, the position of TARA is better regulated towards the desired location by using the proposed hybrid control scheme, in comparison with using the PD/PID control.

\subsection{Summary of Contributions}

- A major contribution of this study, as identified in the literature review, is filling in the gap in current research by simulating and experimentally verifying the flight performance of a quadrotor in a realistic urban wind environment.

- The simulations conducted in this research disclose pertinent information about 
how particular wind characteristics selectively impact quadrotor flight performance for varying control methodologies, allowing for the systematic identification and classification of relevant urban wind phenomena. For instance, contrary to simple intuition, lower mean velocity winds exhibiting turbulent gusts prove to pose more of a position hold challenge to a proportional integral derivative controller than sustained higher velocity winds.

- A comparative analysis of the flight performances of proportional integral derivative, integral backstepping, fuzzy and adaptive integral backstepping in the wake of a building is presented. To date, no study has been conducted placing these controls in the complex flowfield specific to urban areas. The analysis presented here identifies the strengths and weaknesses of each control technique in dealing with such disturbances, as underscored by the selected performance metrics.

- A quadrotor UAV prototype named TARA is developed for the experimental validation of flight simulations. TARA incorporates two critical features for urban deployment, a position control with a sub-GPS accuracy along with sense and avoid capabilities.

- A novel position control technique tailored for the urban wind environment is proposed for the flight control of a quadrotor which demonstrates improvement in both simulation and experimental flights. An average position hold within a single body length is achieved.

\subsection{Future Research}

- Having established a quadrotor simulator based on the airflow around a single building, the urban wind representation can be further enhanced with more 
complicated scenarios, including urban canyons.

- As shown in the literature review, the online estimation of wind disturbances on UAVs is at a beginning stage of research. The developed quadrotor simulator with urban wind provides an ideal platform for testing and evaluating such estimation techniques.

- Based on the characterization of urban airflows, as well as control performance within the simulated urban environment, an improved waypointing system can be created for existing flight controllers, flagging potential danger zones and highlighting regions of expected good performance. 


\section{List of References}

[1] I. Kroo and P. Kunz, "Development of the mesicopter: A miniature autonomous rotorcraft," in American Helicopter Society (AHS) Vertical Lift Aircraft Design Conference, San Francisco, CA, American Helicopter Society, International, 2000.

[2] D. Mellinger, N. Michael, and V. Kumar, "Trajectory generation and control for precise aggressive maneuvers with quadrotors," The International Journal of Robotics Research, vol. 31, pp. 664-674, April 2012.

[3] F. Fraundorfer, L. Heng, D. Honegger, G. Lee, L. Meier, P. Tanskanen, and M. Pollefeys, "Vision-based autonomous mapping and exploration using a quadrotor MAV," in Intelligent Robots and Systems (IROS), 2012 IEEE/RSJ International Conference on, pp. 4557-4564, Oct 2012.

[4] R. Ritz, M. Müller, M. Hehn, and R. D'Andrea, "Cooperative quadrocopter ball throwing and catching," in Intelligent Robots and Systems (IROS), 2012 IEEE/RSJ International Conference on, pp. 4972-4978, Oct 2012.

[5] M. Muller, S. Lupashin, and R. D'Andrea, "Quadrocopter ball juggling," in Intelligent Robots and Systems (IROS), 2011 IEEE/RSJ International Conference on, pp. 5113-5120, Sept 2011.

[6] Q. Lindsey, D. Mellinger, and V. Kumar, "Construction with quadrotor teams," Autonomous Robots, vol. 33, no. 3, pp. 323-336, 2012.

[7] E. Guizzo, "Aeryon Scout quadrotor spies on bad guys from above." [Online]. http://spectrum.ieee.org/automaton/robotics/military-robots/ aeryon-scout-quadrotor-spies-on-bad-guys-from-above, May 2011.

[8] G. Grenzdörffer, A. Engel, and B. Teichert, "The photogrammetric potential of low-cost UAVs in forestry and agriculture," The International Archives of the 
Photogrammetry, Remote Sensing and Spatial Information Sciences, vol. 31, no. B3, pp. 1207-1214, 2008.

[9] M. Neri, A. Campi, R. Suffritti, F. Grimaccia, P. Sinogas, O. Guye, C. Papin, T. Michalareas, L. Gazdag, and I. Rakkolainen, "SkyMedia - UAV-based capturing of $\mathrm{HD} / 3 \mathrm{D}$ content with WSN augmentation for immersive media experiences," in Multimedia and Expo (ICME), 2011 IEEE International Conference on, pp. 1-6, July 2011.

[10] B. Fung, "Its a bird! Its a plane! Its a drone that makes movies!." [Online]. http://www.washingtonpost.com/blogs/the-switch/wp/2013/08/ 15/its-a-bird-its-a-plane-its-a-drone-that-makes-movies/, August 2013.

[11] N. Michael, S. Shen, K. Mohta, Y. Mulgaonkar, V. Kumar, K. Nagatani, Y. Okada, S. Kiribayashi, K. Otake, K. Yoshida, K. Ohno, E. Takeuchi, and S. Tadokoro, "Collaborative mapping of an earthquake-damaged building via ground and aerial robots," Journal of Field Robotics, vol. 29, no. 5, pp. 832-841, 2012.

[12] M.-A. Russon, "Pie in the sky: How Russian firm DoDo Pizza delivers takeaways by drone." [Online]. http://www.ibtimes.co.uk/ drone-delivery-russias-airborne-pizzas-are-height-fine-dining-1454684, June 2013.

[13] E. Ackerman, "When drone delivery makes sense." [Online]. http://spectrum.ieee.org/automaton/robotics/aerial-robots/ when-drone-delivery-makes-sense, September 2014.

[14] Ascending Technologies GmbH, "AscTec Hummingbird." [Online]. http: //www.asctec.de/en/uav-uas-drone-products/asctec-hummingbird/ \#pane-0-0, 2015.

[15] DJI, "DJI Phantom2." [Online]. http://www.dji.com/product/phantom-2, 2015.

[16] AR.Drone, "Parrot." [Online]. http://ardrone2.parrot.com/, 2015.

[17] 3D Robotics Inc., "IRIS+." [Online]. http://3drobotics.com/iris/?_ga=1. 251182659.453616716.1423674260, 2015.

[18] Blade, "350 QX3: Blade." [Online]. http://www.bladehelis.com/350QX3/, 2015. 
[19] Walkera Technology Co., "Scout X4." [Online]. http://www.walkera.com/en/ showgoods . php?id=2292, 2015.

[20] 3D Robotics Inc., "Arducopter.." [Online]. http://copter.ardupilot.com/, 2015.

[21] Transport Canada Advisory Circular, "General safety practices Model aircraft and unmanned air vehicle systems, AC no. 600002." [Online]. http://www.tc.gc.ca/eng/civilaviation/opssvs/ managementservices-referencecentre-acs-600-2135.html, November 2014 .

[22] M. McNulty, "New video: Drone crash lands in Manhattan." [Online]. http://nypost.com/2013/10/03/ video-captures-drones-flight-above-manhattan/, October 2013.

[23] J. Millitzer, "No criminal charges for man who crashed drone into a St. Louis building." [Online]. http://fox2now.com/2014/06/04/ faa-no-criminal-charges-for-man-who-crashed-drone-into-a-st-louis-building/, June 2014.

[24] C. News, "Drone crash prompts Vancouver to review film industry use." [Online]. http://www.cbc.ca/news/canada/british-columbia/ drone-crash-prompts-vancouver-to-review-film-industry-use-1. 2671977, June 2014.

[25] J. G. Leishman, "The Breguet-Richet quad-rotor helicopter of 1907." [Online]. http://www . enae. umd. edu/AGRC/Aero/Breguet.pdf, 1907.

[26] J. Rumerman, "Helicopter development in the early twentieth century." [Online]. www. centennialofflight.gov, December 2002.

[27] S. L. Waslander, G. M. Hoffmann, J. S. Jang, and C. J. Tomlin, "Multi-agent quadrotor testbed control design: integral sliding mode vs. reinforcement learning," 2005 IEEE/RSJ International Conference on Intelligent Robots and Systems, pp. 468-473, 2005.

[28] J. How, B. Bethke, A. Frank, and J. V. D. Dale, "Real-time indoor autonomous vehicle test environment," IEEE Control Systems Magazine, March 2008.

[29] B. Bethke, M. Valenti, and J. How, "Cooperative vision based estimation and tracking using multiple UAVs," in Conference of Cooperative Control and Optimization, (Gainesville, FL), January 2007. 
[30] N. Michael, D. Mellinger, Q. Lindsey, and V. Kumar, "The GRASP multiple micro-UAV testbed," Robotics Automation Magazine, IEEE, vol. 17, pp. 56-65, September 2010.

[31] S. Bouabdallah, Design and control of quadrotors with application to autonomous flying. PhD thesis, Swiss Federal Institute of Technology, 2007.

[32] P. Pounds, R. Mahony, P. Hynes, and J. Roberts, "Design of a four-rotor aerial robot," in Australasian Conference on Robotics and. Automation, (Auckland, New Zealand), November 2002.

[33] P. Pounds, R. Mahony, and J. Gresham, "Towards dynamically-favourable quad-rotor aerial robots," in Australasian Conference on Robotics and Automation, (Canberra, Australia), 2004.

[34] P. Pounds, R. Mahony, and P. Corke, "Modelling and control of a quad-rotor robot," in Australasian Conference on Robotics and Automation, (Auckland, New Zealand), December 2006.

[35] P. Pounds, R. Mahony, and P. Corke, "Modelling and control of a large quadrotor robot," Control Engineering Practice, vol. 18, pp. 691-699, July 2010.

[36] A. Sanchez, L. R. G. Carrillo, E. Rondon, R. Lozano, and O. Garcia, "Hovering flight improvement of a quad-rotor mini UAV using brushless dc motors," Journal of Intelligent and Robotic Systems, vol. 61, pp. 85-101, September 2011.

[37] D. Lara, G. Romero, A. Sanchez, R. Lozano, and A. Guerrero, "Robustness margin for attitude control of a four rotor mini-rotorcraft: Case of study," Mechatronics, vol. 20, p. 143152, 2010.

[38] A. Tayebi and S. McGilvray, "Attitude stabilization of a VTOL quadrotor aircraft," IEEE Transaction on Control System Technology, vol. 14, pp. 562-571, May 2006.

[39] S. Park, D. Won, M. Kang, T. Kim, H. Lee, and S. Kwon, "RIC (robust internal-loop compensator) based flight control of a quad-rotor type UAV," in 2005 IEEE/RSJ International Conference on Intelligent Robots and Systems, pp. 3542-3547, IEEE, August 2005.

[40] J. Kim, M. Kang, and S. Park, "Accurate modeling and robust hovering control for a quadrotor VTOL aircraft," Journal of Intelligent and Robotic Systems, vol. 57, pp. 9-26, 2010. 
[41] S. D. Hanford, "A small semi-autonomous rotary-wing unmanned air vehicle," Master's thesis, Pennsylvania State University, December 2005.

[42] S. Hanford, L. Long, and J. Horn, "A small semi-autonomous rotary-wing unmanned air vehicle (UAV)," in Infotech@Aerospace Conference, (Washington, D.C), AIAA, September 2005. Paper No. 2005-7077.

[43] G. M. Hoffmann, H. Huang, S. Waslander, and C. Tomlin, "Quadrotor helicopter flight dynamics and control: Theory and experiment," in Proceedings of the AIAA Guidance, Navigation, and Control Conference, American Institute of Aeronautics and Astronautics, 2007.

[44] G. Hoffmann, S. Waslander, and C. Tomlin, "Quadrotor helicopter trajectory tracking control," in 2008 AIAA Guidance, Navigation and Control Conference and Exhibit, (Honolulu, Hawaii, USA), August 2008.

[45] M. Tarhan and E. Altug, "EKF based attitude estimation and stabilization of a quadrotor UAV using vanishing points in catadioptric images," Journal of Intelligent and Robotic Systems, vol. 61, no. 1-4, pp. 119-134, 2011.

[46] M. Orsag and S. Bogdan, "Hybrid control of quadrotor," in 17th Mediterranean Conference on Control and Automation, (Thessaloniki, Greece), pp. 1239-1244, IEEE, June 2009.

[47] A. Salih, M. Moghavvemi, H. Mohamed, and K. Gaeid, "Modelling and PID controller design for a quadrotor unmanned air vehicle," in 2010 IEEE International Conference on Automation Quality and Testing Robotics, (Cluj-Napoca), pp. 1-5, IEEE, July 2010.

[48] K. Weng and M. Shukri, "Design and control of a quad-rotor flying robot for aerial surveillance," in 4th Student Conference on Research and Development, (Shah Alam, Selangor, Malaysia,), pp. 173-177, IEEE, June 2006.

[49] S. Fowers, D. Lee, B. Tippetts, K. Lillywhite, A. Dennis, and J. Archibald, "Vision aided stabilization and the development of a quad-rotor micro UAV," in International Symposium on Computational Intelligence in Robotics and Automation, (Jacksonville, Florida), pp. 143-148, IEEE, June 2007.

[50] Z. Ceren and E. Altug, "Vision-based servo control of a quadrotor air vehicle," in Proceedings of the 8th IEEE international conference on Computational intelligence in robotics and automation, (Korea), pp. 84-89, IEEE, December 2009 . 
[51] D. Cabecinhas, C. Silvesstre, and R. Cunha, "Vision-based quadrotor stabilization using a pan and tilt camera," in 49th IEEE Conference on Decision and Control, (Atlanta, GA, USA), pp. 1644-1649, IEEE, December 2010.

[52] K. Wenzel, P. Rosset, and A. Zell, "Low-cost visual tracking of a landing place and hovering flight control with a microcontroller," Journal of Intelligent and Robotic Systems, vol. 57, p. 297311, 2010.

[53] K. Wenzel, A. Masselli, and A. Zell, "Automatic take off, tracking and landing of a miniature UAV on a moving carrier vehicle," Journal of Intelligent and Robotic Systems, vol. 61, p. 221238, 2011.

[54] Q. Zhou, Y. Zhang, C. Rabbath, and D. Theilliol, "Design of feedback linearization control and reconfigurable control allocation with application to a quadrotor UAV," in 2010 Conference on Control and Fault-Tolerant Systems, (Nice), pp. 371-376, IEEE, October 2010.

[55] H. Huang, G. Hoffmann, S. Waslander, and C. Tomlin, "Aerodynamics and control of autonomous quadrotor helicopters in aggressive maneuvering," in 2009 IEEE International Conference on Robotics and Automation, (Kobe, Japan), pp. 3277-3282, IEEE, 2009.

[56] S. Bouabdallah, P. Murrieri, and R. Siegwart, "Design and control of an indoor micro quadrotor," in Proceedings of the International Conference on Robotics and Automation, 2004.

[57] A. Dzul, P. Castillo, and R. Lozano, "Real-time stabilization and tracking of a four-rotor mini rotorcraft," IEEE Transaction on Control System Technology, vol. 12 , no. 4, pp. 510-516, 2004.

[58] E. de Vries and K. Subbarao, "Backstepping based nested multi-loop control laws for a quadrotor," in 11th International Conference on Control Automation Robotics and Vision, (Singapore), pp. 1911-1916, IEEE, December 2010.

[59] S. Bouabdallah and R. Siegwart, "Full control of a quadrotor," in Proceedings of the 2007 IEEE/RSJ International Conference on Intelligent Robots and Systems, (San Diego, CA, USA), pp. 153-158, IEEE, October 2007.

[60] F. Hoffmann, N. Goddemeier, and T. Bertram, "Attitude estimation and control of a quadrocopter," in 2010 IEEE/RSJ International Conference on Intelligent Robots and Systems, (Taipei, Taiwan), pp. 1072-1077, IEEE, October 2010. 
[61] J. Colorado, A. Barrientos, A. Martinez, B. Lafaverges, and J. Valente, "Miniquadrotor attitude control based on hybrid backstepping and Frenet-Serret theory," in 2010 IEEE International Conference on Robotics and Automation (IEEE, ed.), (Anchorage, Alaska, USA), pp. 1617-1622, IEEE, May 2010.

[62] A. Mian and W. Daobo, "Modeling and backstepping-based nonlinear control strategy for a 6 DOF quadrotor helicopter," Chinese Journal of Aeronautics, vol. 21, pp. 261-268, 2008.

[63] P. Adigbli, C. Grand, J. Mouret, and S. Doncieux, "Nonlinear attitude and position control of a micro quadrotor using sliding mode and backstepping techniques," in 3rd US-European Competition and Workshop on Micro Air Vehicle Systems (MAV07) and European Micro Air Vehicle Conference and Flight Competition (EMAV2007),, (Toulouse, France), pp. 1-9, September 2007.

[64] T. Hamel and R. Mahony, "Image based visual servo control for a class of aerial robotic systems," Automatica, vol. 43, p. 19751983, 2007.

[65] N. Guenard, T. Hamel, and V. Moreau, "Dynamic modeling and intuitive control strategy for an X4-flyer," in International Conference on Control and Automation, (Budapest, Hungary), pp. 141-146, IEEE, June 2005.

[66] N. Guenard, T. Hamel, and R. Mahony, "A practical visual servo control for an unmanned aerial vehicle," IEEE Transactions on Robotics, vol. 24, pp. 331-340, April 2008.

[67] Z. Zuo, "Trajectory tracking control design with command-filtered compensation for a quadrotor," IET Control Theory and Applications, vol. 4, no. 11, p. $23432355,2010$.

[68] J. Guerrero-Castellanos, N. Marchand, A. Hably, S. Lesecq, and J. Delamare, "Bounded attitude control of rigid bodies: Real-time experimentation to a quadrotor mini-helicopter," Control Engineering Practice, 2011.

[69] E. Altug, J. Ostrowski, and R. Mahony, "Control of a quadrotor helicopter using visual feedback," in Proceedings of the 2002 IEEE International Conference on Robotics and Automation, (Washington), pp. 72-77, IEEE, May 2002.

[70] G. Hoffmann, D. Rajnarayan, S. Waslander, D. Dostal, J. Jang, and C. Tomlin, "The Stanford testbed of autonomous rotorcraft for multi agent control (STARMAC)," in Proceedings of the 23rd Digital Avionics Systems Conference, (Salt Lake City, Utah), November 2004. 
[71] R. Zhang, X. Wang, and K. Cai, "Quadrotor aircraft control without velocity measurements," in 48th IEEE Conference on Decision and Control, (Shanghai, China), pp. 5213-5218, IEEE, December 2009.

[72] R. Xu and U. Ozguner, "Sliding mode control of a class of underactuated systems," Automatica, vol. 44, pp. 233-241, 2008.

[73] H. Bouadi, M. Bouchoucha, and M. Tadjine, "Sliding mode control based on backstepping approach for an UAV type-quadrotor," International Journal of Mathematical and Computer Sciences, vol. 4, no. 1, pp. 12-17, 2008.

[74] M. Efe, "Robust low altitude behavior control of a quadrotor rotorcraft through sliding modes," in 15th Mediterranean Conference on Control and Automation, (Athens, Greece), July 2007.

[75] A. Benallegue, A. Mokhtari, and L. Fridman, "High-order sliding-mode observer for a quadrotor UAV," International Journal of Robust and Nonlinear Control, vol. 18, no. 4-5, pp. 427-440, 2008.

[76] S. Bouabdallah, A. Noth, and R. Siegwart, "PID vs LQ control techniques applied to an indoor micro quadrotor," in 2004 IEEE/RSJ International Conference on Intelligent Robots and Systems, pp. 2451-2456, October 2004.

[77] I. Cowling, O. Yakimenko, J. Whidborne, and A. Cooke, "Direct method based control system for an autonomous quadrotor," Journal of Intelligent and Robotic Systems, vol. 60, no. 2, pp. 285-316, 2010.

[78] K. Alexis, G. Nikolakopoulos, and A. Tzes, "Constrained optimal attitude control of a quadrotor helicopter subject to wind-gusts: Experimental studies," in American Control Conference, (Baltimore, MD), pp. 4451-4455, IEEE, June 2010.

[79] K. Alexis, G. Nikolakopoulos, and A. Tzes, "Constrained-control of a quadrotor helicopter for trajectory tracking under wind-gust disturbances," in MELECON 2010 - 2010 15th IEEE Mediterranean Electrotechnical Conference, (Valletta, Malta), pp. 1411-1416, IEEE, April 2010.

[80] G. Raffo, M. Ortega, and F. Rubio, "An integral predictive/nonlinear $\mathrm{H}-\infty$ control structure for a quadrotor helicopter," Automatica, vol. 46, pp. 29-39, January 2010. 
[81] L. Kis and B. Lantos, "Quadrotor control based on partial sensor data," in 19th International Workshop on Robotics in Alpe-Adria-Danube Region, (Budapest, Hungary), pp. 43-48, IEEE, June 2010.

[82] A. Mokhtari, A. Benallegue, and B. Daachi, "Robust feedback linearization and GH- $\infty$ controller for a quadrotor unmanned aerial vehicle," in 2005 IEEE/RSJ International Conference on Intelligent Robots and Systems, pp. 1009-1014, IEEE, 2005.

[83] M. Chen and M. Huzmezan, "A combined MBPC/ 2 DOF H- $\infty$ controller for a quad rotor UAV," in AIAA Guidance, Navigation, and Control Conference and Exhibit, (Austin, Texas), August 2003.

[84] M. Huang, B. Xian, C. Diao, K. Yang, and Y. Feng, "Adaptive tracking control of underactuated quadrotor unmanned aerial vehicles via backstepping," in American Control Conference, (Baltimore, MD), pp. 2076-2081, IEEE, June 2010 .

[85] T. Madani and A. Benallegue, "Adaptive control via backstepping technique and neural networks of a quadrotor helicopter," in Proceedings of the 17th World Congress The International Federation of Automatic Control, (Seoul, Korea), pp. 6513-6518, July 2008.

[86] C. Nicol, C. Macnab, and A. Ramirez-Serrano, "Robust adaptive control of a quadrotor helicopter," Mechatronics, 2011.

[87] C. Coza and C. Macnab, "A new robust adaptive-fuzzy control method applied to quadrotor helicopter stabilization," in Annual meeting of the North American Fuzzy Information Processing Society, (Montreal), pp. 454-458, IEEE, June 2006.

[88] K. Zemalache and H. Maaref, "Controlling a drone: Comparison between a based model method and a fuzzy inference system," Applied Soft Computing, vol. 9, p. 553562, 2009.

[89] D. Lee, H. J. Kim, and S. Sastry, "Feedback linearization vs. adaptive sliding mode control for a quadrotor helicopter," International Journal of Control, Automation, and Systems, vol. 7, no. 3, pp. 419-428, 2009.

[90] S. A. Raza and W. Gueaieb, "Fuzzy logic based quadrotor flight controller," In Proceedings of the International Conference on Informatics in Control, Automation and Robotics (ICINCO), July 2-5 2009. 
[91] M. Santos, V. Lopez, and F. Morata, "Intelligent fuzzy controller of a quadrotor," in 2010 International Conference on Intelligent Systems and Knowledge Engineering, (Hangzhou), pp. 141-146, IEEE, November 2010.

[92] K. Yapici, "Self-ruled fuzzy logic based controller," in The 5th IFAC Intl. WS DECOM-TT, (Cesme, Turkey), IFAC, May 2007.

[93] Y. Al-Younes and M. A. Jarrah, "Attitude stabilization of quadrotor UAV using backstepping fuzzy logic and backstepping least-mean-square controllers," in 5th International Symposium on Mechatronics and Its Applications, (Amman, Jordan), pp. 1-11, IEEE, May 2008.

[94] H. Bou-Ammar, H. Voos, and W. Ertel, "Controller design for quadrotor UAVs using reinforcement learning," in 2010 IEEE International Conference on Control Applications, (Yokohama, Japan), pp. 2130-2135, IEEE, September 2010.

[95] O. Purwina and R. D'Andrea, "Performing and extending aggressive maneuvers using iterative learning control," Robotics and Autonomous Systems, vol. 59, pp. 1-11, 2011.

[96] T. Dierks and S. Jagannathan, "Output feedback control of a quadrotor UAV using neural networks," IEEE Transactions on Neural Networks, vol. 21, pp. 5065, January 2010.

[97] M. Efe, "Neural network assisted computationally simple $\mathrm{PI}^{\lambda} \mathrm{D}^{\mu}$ control of a quadrotor UAV," IEEE Transactions on Industrial Informatics, vol. 7, pp. 354361, April 2011.

[98] A. Das, F. Lewis, and K. Subbarao, "Backstepping approach for controlling a quadrotor using lagrange form dynamics," Journal of Intelligent and Robotic Systems, vol. 56, pp. 127-151, September 2009.

[99] P. Castillo, A. Dzul, and R. Lozano, "Real-time stabilization and tracking of a four-rotor mini rotorcraft," IEEE Transactions on Control Systems Technology, vol. 12, pp. 510-516, July 2004.

[100] M. Orr, S. Rasmussen, E. Karni, and W. Blake, "Framework for developing and evaluating MAV control algorithms in a realistic urban setting," American Control Conference, June 8-10 2005.

[101] D. Galway, J. Etele, and G. Fusina, "Modeling of urban gust environment with application to autonomous flight," 35th AIAA Atmospheric Flight Mechanics Conference and Exhibit, August 18-21 2008. 
[102] D. Galway, J. Etele, and G. Fusina, "Modeling of urban wind field effects on unmanned rotorcraft flight," AIAA Journal of Aircraft, vol. 1, no. 1, pp. 100$108,2010$.

[103] M. Crump, C. Bil, and P. Riseborough, "An autonomous control technique for launching ship based unmanned air vehicles (UAVs) in extreme conditions," in 23rd ICAS Congress, pp. 8-13, 2002.

[104] F. Wong, E. Gagnon, K. Mctaggart, S. Zan, J. Colwell, and O. Theiault, "A hardware-in-the-loop study of factors affecting recovery of UAVs on canadian forces frigates," Launch and Recovery of Manned and Unmanned Vehicles from Surface Platforms, American Society of Naval Engineers, May 2008.

[105] J. Horn and D. Bridges, "A model following controller optimized for gust rejection during shipboard operations," in Annual Forum Proceedings - American Helicopter Society, vol. 63, p. 1799, 2007.

[106] N. Sydney, B. Smyth, and D. A. Paley, "Dynamic control of autonomous quadrotor flight in an estimated wind field," in Decision and Control (CDC), 2013 IEEE 52nd Annual Conference on, pp. 3609-3616, IEEE, 2013.

[107] C. Hancer, K. T. Oner, E. Sirimoglu, E. Cetinsoy, and M. Unel, "Robust position control of a tilt-wing quadrotor," in Decision and Control (CDC), 2010 49th IEEE Conference on, pp. 4908-4913, IEEE, 2010.

[108] S. L. Waslander and C. Wang, "Wind disturbance estimation and rejection for quadrotor position control," in AIAA Infotech@ Aerospace Conference and AIAA Unmanned... Unlimited Conference, Seattle, WA, 2009.

[109] K. Alexis, G. Nikolakopoulos, Y. Koveos, and A. Tzes, "Switching model predictive control for a quadrotor helicopter under severe environmental flight conditions," in World Congress, vol. 18, pp. 11913-11918, 2011.

[110] K. Alexis, G. Nikolakopoulos, and A. Tzes, "Model predictive quadrotor control: attitude, altitude and position experimental studies," Control Theory \& Applications, IET, vol. 6, no. 12, pp. 1812-1827, 2012.

[111] F.Kendoul, K. Nonami, I. Fantoni, and R. Lozano, "An adaptive vision-based autopilot for mini flying machines guidance, navigation and control," $A u$ tonomous Robots, vol. 27, no. 3, pp. 165-188, 2009. 
[112] N. Guenard, T. Hamel, and R. Mahony, "A practical visual servo control for a unmanned aerial vehicle," in 2007 IEEE International Conference on Robotics and Automation, (Rome, Italy), pp. 1342-1348, IEEE, April 2007.

[113] O. Bourquardez, N. Guenard, T. Hamel, F. Chaumette, R. Mahony, and L. Eck, "Kinematic visual servo controls of an X4-flyer: Practical study," in 1st Mediterranean Conference on Intelligent Systems and Automation, (Annaba, Algeria), pp. 391-396, 2008.

[114] O. Bourquardez, R. Mahony, N. Guenard, F. Chaumette, T. Hamel, and L. Eck, "Image-based visual servo control of the translation kinematics of a quadrotor aerial vehicle," IEEE Transactions on Robotics, vol. 25, pp. 743-749, June 2009.

[115] P. Kastner-Klein, R. Berkowicz, and R. Britter, "The influence of street architecture on flow and dispersion in street canyons," Meteorology and Atmospheric Physics, vol. 87, 2004.

[116] J. Boris, "Dust in the wind: Challenges for urban aerodynamics," 35th AIAA Fluid Dynamics Conference and Exhibit, June 6-9 2005.

[117] S. Murakami, R. Ooka, A. Mochida, S. Yoshida, and S. Kim, "CFD analysis of wind climate from human scale to urban scale," Journal of Wind Engineering and Industrial Aerodynamics, vol. 81, 1999.

[118] C. S. J. He, "Evaluation of pedestrian winds in urban area by numerical approach," Journal of Wind Engineering and Industrial Aerodynamics, vol. 81, 1999.

[119] A. V. P. Neofytou, D. Vlachogiannis, J. Bartzis, and A. Scaperdas, "CFD simulations of the wind environment around an airport terminal building," Environmental Modelling Software, vol. 21, 2006.

[120] P. Oliveira and B. Younis, "On the prediction of turbulent flows around fullscale buildings," Journal of Wind Engineering and Industrial Aerodynamics, vol. 86, 2000.

[121] G. Patnaik, J. Boris, F. Grinstein, and J. Iselin, "Large scale urban simulations with FCT," Scientific Computation, 2005.

[122] G. Panaik, J. Boris, and F. Grinstein, "Large scale urban simulations with the MILES approach," 16th AIAA Computational Fluid Dynamics Conference, June 23-26 2003. 
[123] A. Baskaran and A. Kashef, "Investigation of air flow around buildings using computational fluid dynamics techniques," Engineering Structures, vol. 18, 1996.

[124] D. Lakehal and W. Rodi, "Calculation of the flow past a surface-mounted cube with two-layer turbulence models," Journal of Wind Engineering and Industrial Aerodynamics, vol. 67, 1997.

[125] R. Meroney, B. Leitl, S. Rafailidis, and M. Schatzmann, "Wind-tunnel and numerical modeling of flow and dispersion about several building shapes," Journal of Wind Engineering and Industrial Aerodynamics, vol. 81, 1999.

[126] E. Simiu and R. H. Scanlan, Wind Effects on Structures. New York, NY, USA: John Wiley and Sons, Inc., 3rd ed., 1996.

[127] K. Elshorbagy, E. Wahba, and R. Afify, "Visualization versus CFD simulation of laminar flow past bluff body," Proceedings of Tenth International Congress of Fluid Dynamics, 2010.

[128] S. Krajnovic and L. Davidson, "Flow around a three-dimensional bluff body," 9th International Symposium on Flow Visualization, 2000.

[129] J. S. Ochoa and N. Fueyo, "Large eddy simulation of the flow past a square cylinder," PHOENICS 10th International User Conference, 2004.

[130] M. Sutherland, "Urban wake field generation using les for application to quadrotor flight," Master's thesis, Department of Mechanical and Aerospace Engineering, Carleton University, May 2015.

[131] OpenCFD Ltd, "OpenFOAM: The open source CFD toolbox." [Online]. http: //www. openfoam.com/, June 2014.

[132] H. Versteeg and W. Malalasekera, An Introduction to Computational Fluid Dynamics: The Finite Volume Method. Harlow, England, New York: Prentice Hall, 2nd ed., January 2007.

[133] S. Winkvist, E. Rushforth, and K. Young, "Towards an autonomous indoor aerial inspection vehicle," Industrial Robot: An International Journal, vol. 40, no. 3, pp. 196-207, 2013.

[134] V. A. Photos and Surveys, "Professional UAV/drone aerial photography and innovations Victoria BC." [Online]. http://victoriaairphotos.com/roof/ _inspections.html. 
[135] A. Albers, S. Trautmann, T. Howard, T. A. Nguyen, M. Frietsch, and C. Sauter, "Semi-autonomous flying robot for physical interaction with environment," in Robotics Automation and Mechatronics (RAM), 2010 IEEE Conference on, pp. 441-446, June 2010.

[136] J. Capitan, D. Mantecon, P. Soriano, and A. Ollero, "Autonomous perception techniques for urban and industrial fire scenarios," in Safety, Security and Rescue Robotics, 200\%. IEEE International Workshop on, pp. 1-6, Sept 2007.

[137] C. Hoyos, "UAVs in cinematography," AIAA 2nd Unmanned Unlimited, Systems Technologies and Operations - Aerospace, Land, and Sea Conference, Workshop, and Exhibition, September 15-18 2003.

[138] B. Guerreiro, C. Silvestre, A. Cunha, and D. Guerreiro Tome Antunes, "Trajectory tracking $\mathrm{H} 2$ controller for autonomous helicopters: and application to industrial chimney inspection," Proceedings of the 17th IFAC Symposium on Automatic Control in Aerospace, June 25-29 2007.

[139] H. Khalil, Nonlinear Systems. Prentice Hall, 3rd ed., 2002.

[140] Electrifly, "Rimfire 400 brushless DC motors." [Online]. http://www . electrifly.com/motors/gpmg4505.html, 2014.

[141] DJI Inc., "DJI Series 30 A OPTO ESC." [Online]. http://wiki.dji.com/en/ index.php/ESC, 2014.

[142] APC Propellers, "APC 10x4.7 SPF counter rotating propeller." [Online]. http: //www . apcprop. com/ProductDetails . asp?ProductCode=LP10047SFP, 2014.

[143] STMicroelectronics, "LIS3L02AL triple axis accelerometer." [Online]. http: //www.st.com/web/en/catalog/sense_power/FM89/SC444, 2014.

[144] InvenSense, "IDG-600 based triple axis gyroscope." [Online]. http:// invensense.com/mems/gyro/dualaxis.html, 2014.

[145] Honeywell, "HMC5883L digital compass module." [Online]. http:// aerospace.honeywell.com/en/products/sensors/non-inertial-sensors/ magnetic-field-sensing-and-sensor-solutions/ featured-product-hmc58831, 2014.

[146] Maxbotix, "MaxBotix MB1340 sonar sensor." [Online]. http://www . maxbotix.com/Ultrasonic_Sensors/MB1340.htm, 2014. 
[147] Micro Modular Technologies, "MN5010HS micro-mini GPS module." [Online]. http://www.micro-modular.com/products/gps/MN5010HS, 2014.

[148] Society of Robots, "Axon ATMega640 based controller board." [Online]. http: //www.societyofrobots.com/axon/, 2014.

[149] Arduino, "Arduino Pro Mini 328 board." [Online]. http://arduino.cc/en/ Main/ArduinoBoardProMini, 2014.

[150] Digi International Inc., "Xbee Pro wireless transciever module." [Online]. http: //www.digi.com/products/model?mid=2580, 2014.

[151] J. Roberts, T. Stirling, J. Zufferey, and D. Floreano, "Quadrotor using minimal sensing for autonomous indoor flight," in European Micro Air Vehicle Conference and Flight Competition, (Toulouse, France), pp. 1-8, September 2007.

[152] K. Y. Chee and Z. W. Zhong, "Control, navigation and collision avoidance for an unmanned aerial vehicle," Sensors and Actuators A: Physical, vol. 190, no. 190, pp. 66-76, 2013.

[153] MaxBotix Inc., "Using multiple MaxSonar sensors simultaneously." [Online]. http://www.maxbotix.com/articles/031.htm, 2012.

[154] MaxBotix Inc., "Ultrasonic sensor operation on a quadcopter." [Online]. http: //www.maxbotix.com/articles/067.htm, 2012. 


\section{Appendix A}

\section{Design and Development of TARA}

A quadrotor UAV prototype called TARA, as shown in Figure 66, is designed and developed in the Mechanical and Aerospace Engineering department at Carleton University. TARA measures $0.43 \mathrm{~m}$ from the tip of one propeller to another, and weighs $2.2 \mathrm{~kg}$, with an extra $500 \mathrm{~g}$ of payload capacity. TARA is able to hover and maintain its altitude and lateral position autonomously, as well as perform flight maneuvers based on commands issued by a human operator using a radio controller. This section presents details of the system design, hardware and software used for implementing an autopilot onboard TARA.

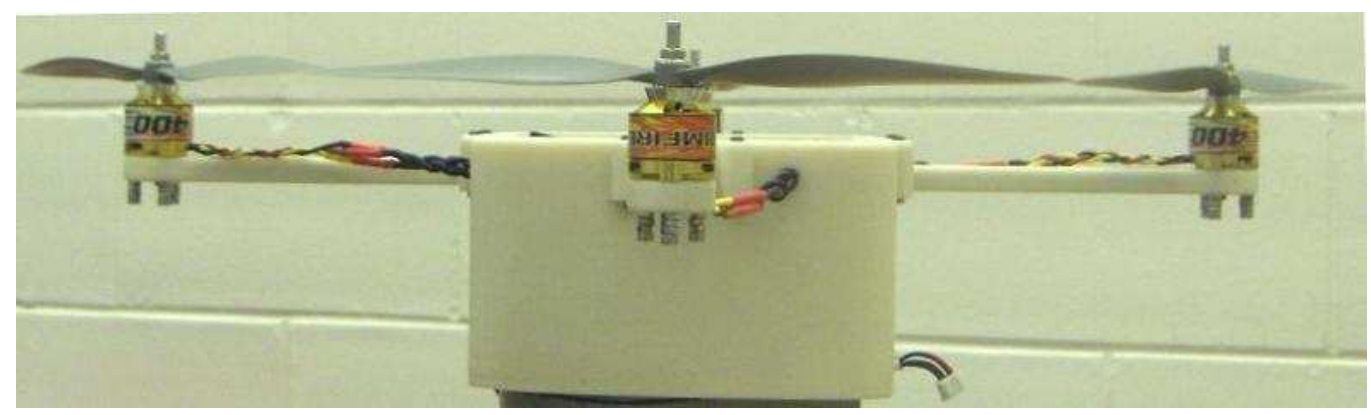

Figure 66: TARA quadrotor UAV prototype.

Figure 67 illustrates TARA's onboard autopilot and its connectivity with various sensors, communication modules, and the actuators. All the components of this system design are explained with detail in the following sub-sections. 


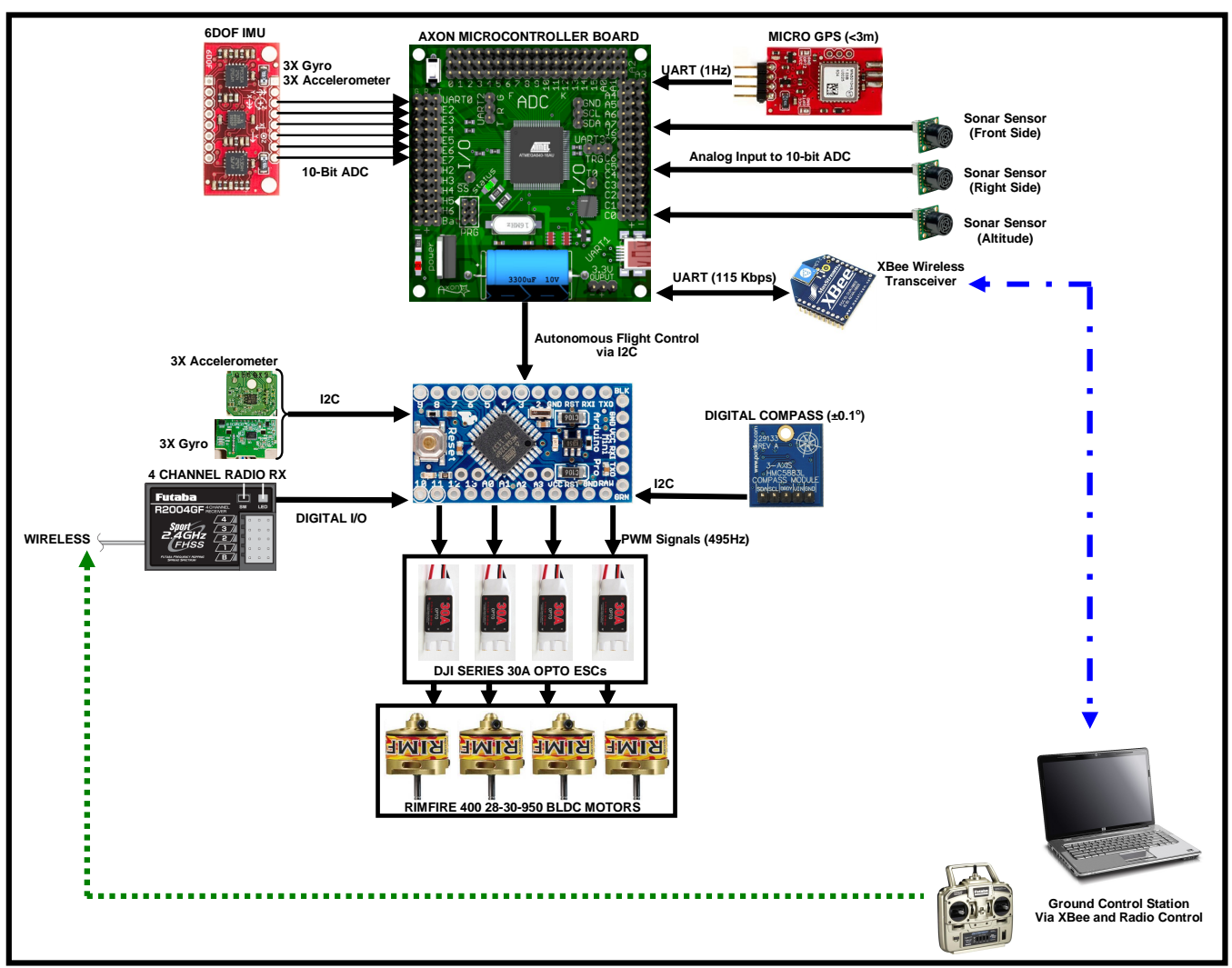

Figure 67: TARA's system design. 


\section{A.1 Airframe Design}

The airframe for the quadrotor, as shown in Figure 68, is designed in a CAD software called Pro Engineer. In conformity with the canonical quadrotor configuration, four arms containing motor mounts at their ends are connected in a plus arrangement. Developed using 3D printing technology, the arms are made of Acrylonitrile Butadiene Styrene (ABS) plastic. To further strengthen the ABS frame, carbon fiber reinforced polymer $(\mathrm{CFRP})$ rods are inserted inside the four arms. The CFRP rods are connected at the airframe center via a plus shaped connector. The resulting airframe is rigid and symmetrical as per the design requirements for a quadrotor. The airframe is sandwiched between a top lid and a bottom chassis to provide shielding to the onboard electronic peripherals. The bottom chassis contains mounting holes for secure installation of the electronic circuits, as well as sockets for safe placement of the batteries.

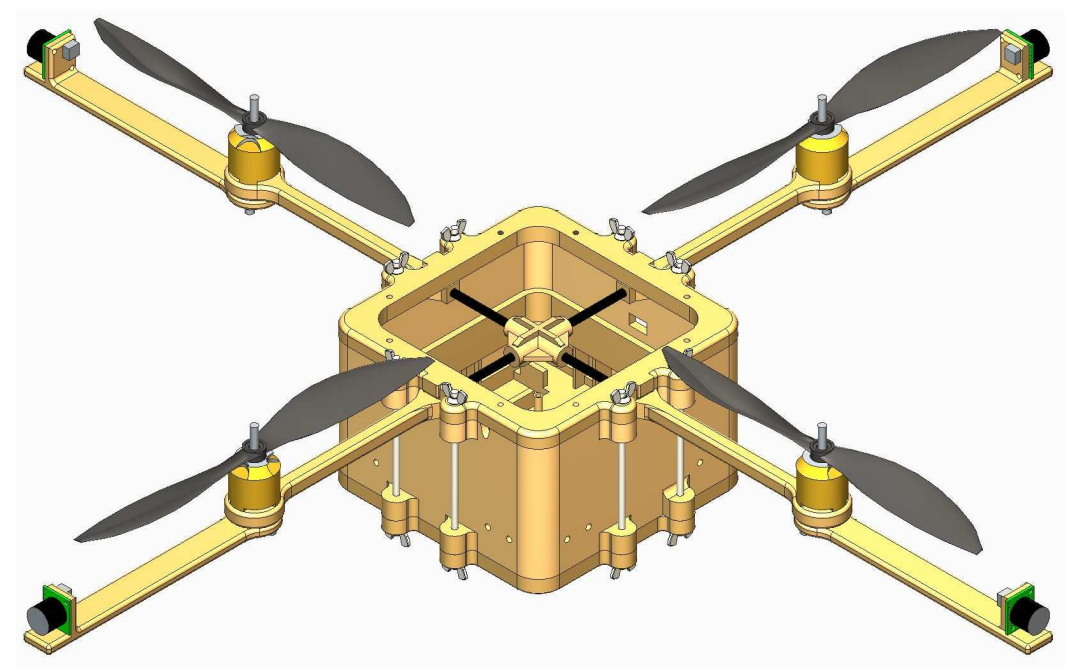

Figure 68: The CAD model of TARA. 


\section{A.2 Propulsion System}

The BLDC motor selected for the quadrotor UAV is the Great Planes RimFire 400, 28-30-950, as shown in Figure 69. The seven digit numbering system provides the basic specifications about the motor. The first two digits represent the diameter of the motor in millimeters, and the next set of digits show the length of motor. The last three digits are the $\mathrm{Kv}(\mathrm{RPM} /$ Volt $)$ rating of the motor. Motors with a lower $\mathrm{Kv}$ rating have a higher number of winding turns and spin slower and can spin a larger propeller consuming less current. Similarly, motors with a higher Kv rating have a low number of winding turns and spin faster with a small propeller but consume higher current. These characteristics emphasize the need for prior analysis on the type of application when selecting appropriate motors. In our case, the quadrotor has four motors and it is mandatory to select motors with lower $\mathrm{Kv}$ rating to conserve maximum power.
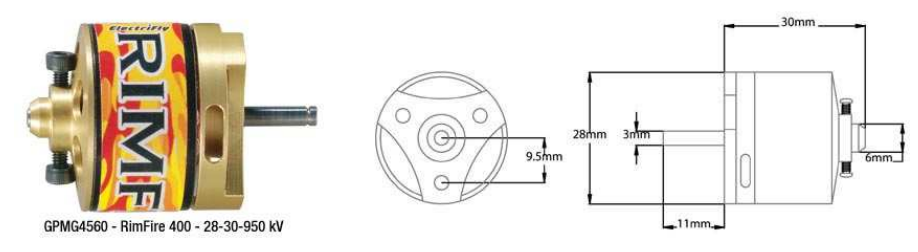

Figure 69: The Rimfire 400, 28-30-950 BLDC motor [140].

The Rimfire 28-30-950 motor weighs $54 \mathrm{~g}$, requires an input voltage of $7.4 \mathrm{~V}$ to 11.1 V, and has a $0.7 \mathrm{~A}$ no-load current. The maximum limit on the current is 14 A to $20 \mathrm{~A}$, where $20 \mathrm{~A}$ is the surge current. The maximum power consumption is 155 Watts, where 220 Watt bursts are possible. The motor can handle a slow flyer propeller size ranging between 8x6 (length $\mathrm{x}$ pitch) to $10 \mathrm{x} 4$ inches.

BLDC motors are a kind of synchronous motor and require a timed sequence of commutation for their operation. This commutation is provided by a microcontroller switching the MOSFETs based on the feedback from the Hall sensors built in the 
motors. The microcontroller and the power commutation circuit are combined to form an Electronic Speed Controller (ESC). The input to this ESC is usually a pulsewidth modulation (PWM) signal sent from the high-level controller to set the desired speed of the motor. An ESC helps to free the main controller from unnecessary performance burden. The ESC requires a standard input PWM signal, as depicted in Figure 70, of period $20 \mathrm{~ms}$ with a duty cycle varying from $1 \mathrm{~ms}$ to $2 \mathrm{~ms}$, used mostly in RF transmitters for servo motor control. The PWM signal is refreshed with a typical $50 \mathrm{~Hz}$ rate which is most common within the hobby class of equipment. Recently, due to the increasing popularity of quadrotors in hobby circles, high speed ESCs are becoming a prefered choice for rotorcraft applications. As the refresh rate of the ESC is increased from a typical $50 \mathrm{~Hz}$ to $400 \mathrm{~Hz}$, and in some cases $1 \mathrm{KHz}$, this allows the autopilot to apply control corrections at matching rates, thus greatly improving the attitude stabilization performance. Other ESC features such as safe start prevent the motor from rotating when the battery is first connected, a mandatory safety precaution for rotorcrafts. An arming routine of specific signals is required every time the battery is connected, in order to arm the motor for functioning.

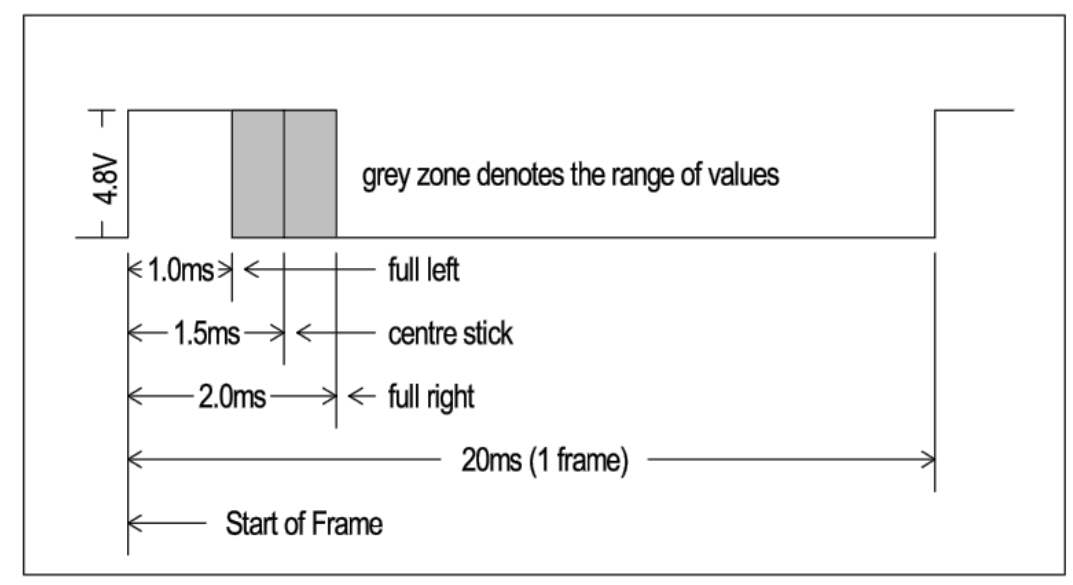

Figure 70: The PWM timing diagram.

The high speed ESC selected for matching the BLDC motor specifications is DJI 
Series 30 A OPTO, shown in Figure 71. It weighs $30 \mathrm{~g}$ and can deliver current from 30 A to a maximum surge limit of $35 \mathrm{~A}$. The input power requirements are 3-4 cells LiPo battery with voltage up to $20 \mathrm{~V}$. The ESC operates at a signal refresh frequency of $450 \mathrm{~Hz}$ and is programmed for safety start of the motors.

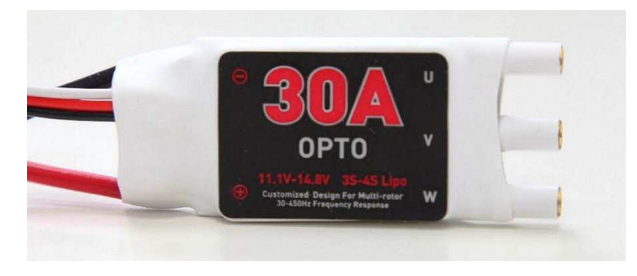

Figure 71: The DJI Series 30 A OPTO ESC [141].

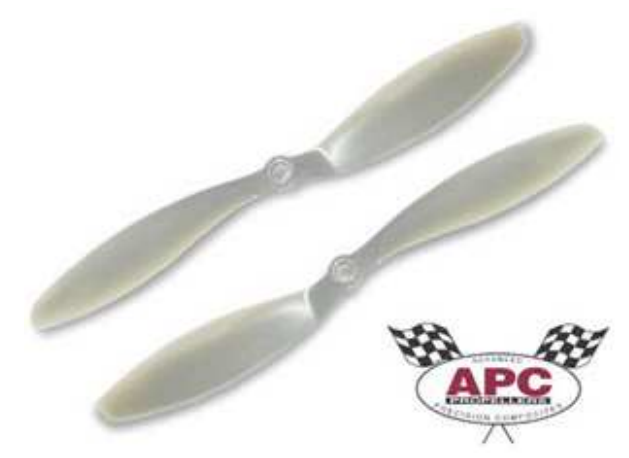

Figure 72: The APC counter rotating propeller pair [142].

The selection of the most efficient propeller requires determining the propeller with the highest thrust to current ratio. The choice of the propeller will reflect on the size, payload capacity and the overall efficiency of the quadrotor propulsion system. Advanced Precision Composites (APC) 10x4.7SFP counter rotating pairs, displayed in Figure 72, are used for TARA's propulsion. Each propeller weighs $11 \mathrm{~g}$ and maintains its rigidity for an RPM limit of 6500. Beyond this limit the propeller keep producing higher thrust, however, with a commensurate loss of performance due to bending. 


\section{A.3 Power Supply}

Lithium Ion Polymer (Li-Po) batteries are very popular for robot applications due to their higher energy density as well as faster charging times. These features offer a significant advantage over their $\mathrm{NiCd}$ and $\mathrm{NiMH}$ counterparts. For a high endurance UAV, longer flight times are associated with the amount of power available onboard. This requirement is resolved by adding batteries in parallel; therefore, two $11.1 \mathrm{~V}$, $2200 \mathrm{mAh}$, Li-Po batteries are use in parallel to power the quadrotor. The resulting power supply offers a high energy density and guarantees an endurance of 15 minutes.

\section{A.4 Orientation Sensors}

For making an autonomous flight possible it is important to have information about the attitude of the quadrotor and its position coordinates in space. Furthermore, to prevent the quadrotor from colliding into obstacles, an obstacle detection technique is also required. The attitude of the quadrotor is determined by implementing an inertial measurement unit based on a triple axis gyroscope, triple axis accelerometer, and dual axis magnetometer.

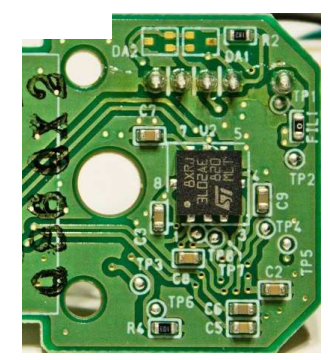

Figure 73: The LIS3L02AL triple axis accelerometer [143].

Inertial sensors such as LIS3L02AL tri-axis accelerometers, Figure 73, with $\pm 2 \mathrm{~g}$ full-scale measurement range and $0.5 \mathrm{mg}$ resolution over $100 \mathrm{~Hz}$ bandwidth, are used for measuring gravity aided pitch and roll angles. The sensor operates on $3.3 \mathrm{~V}$ and 
consumes $0.85 \mathrm{~mA}$ of current during typical operation. The acceleration measurements are made by a 10-bit ADC and output is available via an $\mathrm{I} 2 \mathrm{C}$ communication bus.

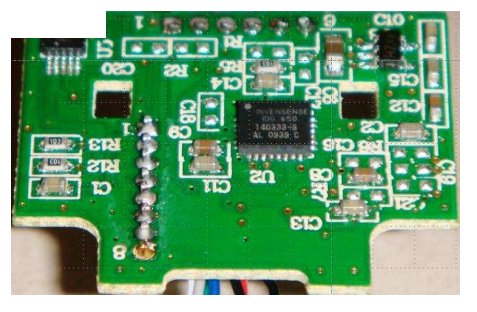

Figure 74: The IDG-600 based triple axis gyroscope [144].

Two dual-axis gyroscopes IDG-600 made by InvenSense, Figure 74, capable of measuring $\pm 500 \mathrm{deg} / \mathrm{sec}$ range with a sensitivity of $3.33 \mathrm{mV} / \mathrm{deg} / \mathrm{sec}$, are used for determining rotational rates for pitch, roll, and yaw axes. The gyroscopes operate on a power supply of $3.3 \mathrm{~V}$ and draw a current of $10 \mathrm{~mA}$ on average. The measured angular rates are 14-bit ADC values that are transmitted via I2C bus.

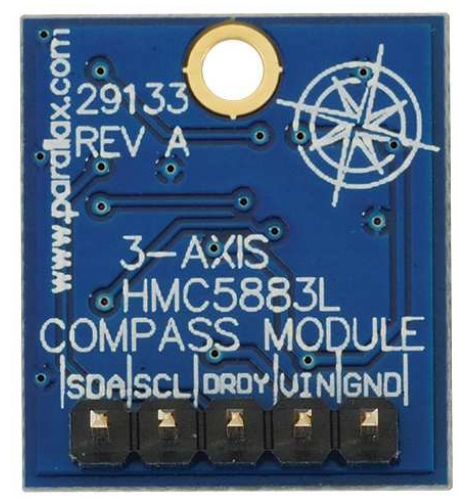

Figure 75: The HMC5883L digital compass module [145].

Based on a triple axis magnetometer, the Honeywell HMC5883L digital compass, Figure 75, with 1 to 2 deg accuracy is used for complementing the yaw angle estimates. The compass operates on a 3.3 V supply and consumes $100 \mu \mathrm{A}$ of current. The heading information is available via $\mathrm{I} 2 \mathrm{C}$ bus at a fast output rate of $160 \mathrm{~Hz}$. 
The sensors are mounted such that the circuits are located exactly at the center of the chassis, placing the IMU as close as possible to the COG of the quadrotor.

\section{A.5 Positioning Sensors}

Reference based positioning: Ultrasonic ranging sensors are employed for range measurements indoors along with obstacle detection and avoidance. The position of TARA is measured using three MaxBotix MB1340 sonar sensors, Figure 76, oriented outwards at each of the four arms, and one pointing downwards for altitude measurement. The MB1340 is a single transducer based range finder weighing only 5.9 g, which can measure distances between $20 \mathrm{~cm}$ to $7 \mathrm{~m}$ with $1 \mathrm{~cm}$ precision. The sonar sensor is fully automatic in its operation, such that once triggered by the main microcontroller it takes care of the whole process of producing several sonic pulses, receiving the echoes and calculating the averaged range measurement. This calculated distance value is transmitted to the main microcontroller, thus minimizing the low level sensor processing burden. The sensor operates at a frequency of $42 \mathrm{kHz}$ with a range reading rate of $10 \mathrm{~Hz}$ and requires a voltage of $5 \mathrm{~V}$ and current supply of $3.4 \mathrm{~mA}$. Three operating modes can be selected to configure the sensor for providing range measurement as an analog output of $(\mathrm{Vcc} / 1024) / \mathrm{cm}$, a PWM signal where the pulse width represents the measured range, or simply the measured range value over a 9600 Baud serial communication standard. In serial communication mode the data is transmitted as pulses of $0-5 \mathrm{~V}$ and can be directly connected to the UART without the need for any RS-232 interface. Up to sixteen ultrasonic rangers can be connected to the UART of the microcontroller in serial bus configuration.

GPS: Position measurements outdoors are obtained from the Global Positioning System (GPS), in the form of latitude, longitude and altitude coordinates. A SiRF 


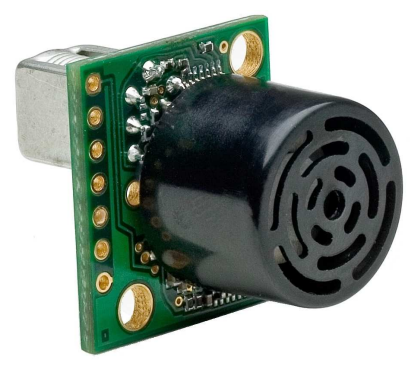

Figure 76: The MaxBotix MB1340 Sonar Sensor [146].

III chipset based Global Positioning System (GPS) module is used to provide the Latitude, Longitude, and Altitude (LLA) coordinates at a rate of $1 \mathrm{~Hz}$ and with an accuracy of $3 \mathrm{~m}$. The GPS receiver used in the quadrotor is the MN5010HS MicroMini module as shown in Figure 77. The GPS operates at a voltage of $5 \mathrm{~V}$ and requires a typical current supply of $36 \mathrm{~mA}$. The sensitivity of the device is $-159 \mathrm{dBm}$ and the position coordinates are updated at a rate of $1 \mathrm{~Hz}$. The position accuracy is less than $10 \mathrm{~m}$ when operating in autonomous mode, and the velocity measurement accuracy is $0.1 \mathrm{~m} / \mathrm{s}$. The GPS data is available through a built-in UART at a 4800 bps data rate.

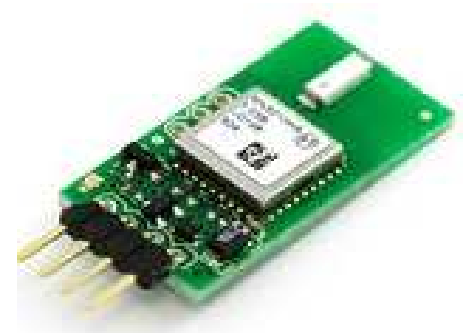

Figure 77: The MN5010HS Micro-Mini GPS module [147].

The GPS data is available in form of National Marine Electronics Association (NMEA) messages. The MN5010HS GPS supports the following NMEA-0183(V3.1) standard messages: 
- GPGGA (Global Positioning System Fixed Data)

- GPGSA (GNSS DOP and Active Satellites)

- GPGSV (GNSS Satellites in view)

- GPRMC (Recommended Minimum Specific GNSS Data)

For the position coordinates GPGGA and GPRMC messages, described in Table 22 and 23, were acquired and parsed for the required data.

Table 22: GPGGA fixed data message template.

\begin{tabular}{|c|c|c|}
\hline Name & Example & Description \\
\hline \hline Message ID & \$GPGGA & GGA protocol header \\
\hline UTC Position & 161229.487 & hhmmss.sss \\
\hline Latitude & 3727.2475 & ddmm.mmmm \\
\hline N/S Indicator & $\mathrm{N}$ & N=north or S=south \\
\hline Longitude & 12158.3416 & dddmm.mmmm \\
\hline E/W Indicator & W & E=east or W=west \\
\hline Position fix indicator & 1 & $0=$ invalid,1=valid,2=DGPS,3=PPS \\
\hline Satellites used & 07 & Range 0 to 12 \\
\hline HDOP & 1.0 & Horizontal Dilution of Precision \\
\hline MSL Altitude (meters) & 9.0 & Altitude above mean-sea-level \\
\hline Units & $\mathrm{M}$ & Units of antenna altitude \\
\hline Geoid Separation (meters) & & Geoidal height \\
\hline Units & $\mathrm{M}$ & Units of geoidal height \\
\hline Age of Diff. Corr. (seconds) & & Age of Differential GPS data \\
\hline Dif. Ref. Station ID & & 0000 to 1023 \\
\hline Checksum & & Check for transmission errors \\
\hline$<~$ & & End message termination \\
\hline
\end{tabular}


Table 23: GPRMC minimum specific GNSS data message template.

\begin{tabular}{|c|c|c|}
\hline Name & Example & Description \\
\hline \hline Message ID & \$GPRC & RMC protocol header \\
\hline UTC Position & 161229.487 & hhmmss.sss \\
\hline Latitude & 3727.2475 & ddmm.mmmm \\
\hline N/S Indicator & $\mathrm{N}$ & N=north or $\mathrm{S}=$ south \\
\hline Longitude & 12158.3416 & dddmm.mmmm \\
\hline E/W Indicator & $\mathrm{W}$ & E=east or W=west \\
\hline Ground Speed (knots) & 0.13 & Speed over ground in knots \\
\hline Ground Course (degrees) & 309.62 & Track made good in degrees True \\
\hline Date & 120598 & ddmmyy, UTC date of fix \\
\hline Mag. Variation (degrees) & & Easterly var. subtracts from true course \\
\hline E/W Indicator & W & E=east or W=west \\
\hline Mode indicator & A & A=Auto.,D=Diff.,E=Est.,N=invalid \\
\hline Status & A & Data status \\
\hline Checksum & $* 10$ & Check for transmission errors \\
\hline$<C R><F>$ & & End of message termination \\
\hline
\end{tabular}




\section{A.6 Onboard Processors}

Two processing boards are used to implement the autopilot for TARA. The highlevel controller is implemented on an ATMega640 microcontroller based platform called Axon developed by Society of Robots. The Axon, as shown in Figure 78, was designed specifically for robotic applications and is one of the most compact and light-weight microcontroller boards. Apart from these physical aspects, Axon offers several features such as 8-bit processing at $16 \mathrm{MHz}, 64 \mathrm{~kb}$ flash and $8 \mathrm{~kb}$ RAM, 16 channels for ADC with 10-bit resolution, 9 PWM channels, 6 timers, 4 UARTs, a USB port, SPI and I2C buses, 55 digital I/O pins, and a power bus with regulated 5 $\mathrm{V}$ and $3.3 \mathrm{~V}$ outputs included on the circuit board. These features clearly set Axon apart from most of the microcontroller boards available in the market.

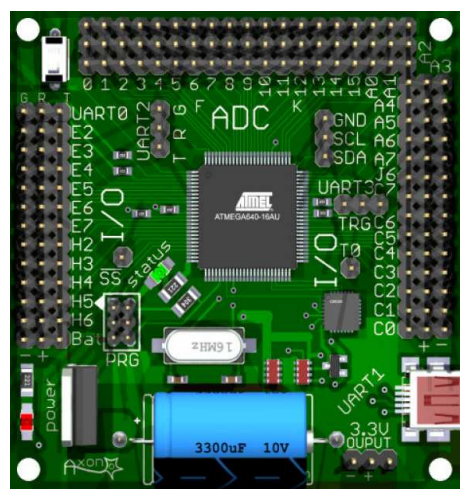

Figure 78: The Axon ATMega640 based controller board [148].

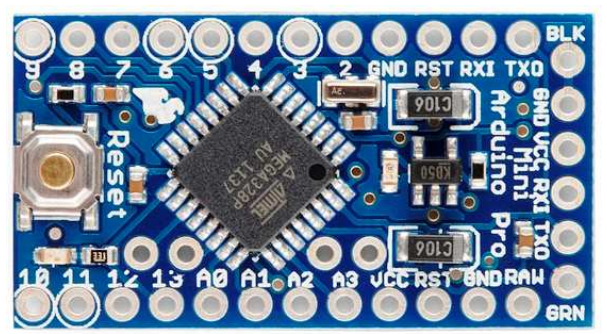

Figure 79: The Arduino Pro Mini 328 board [149]. 
For low-level processing an Arduino Pro Mini 328 board is selected. The Pro Mini 328 board, as shown in Figure 79, is tiny in size and weighs only 2 g. The 8-bit microcontroller runs at $16 \mathrm{MHz}$ and has $8 \mathrm{ADC}$ inputs with a 10-bit resolution. Various features such as PWM channels, UARTs, and I2C are available along with 14 digital I/O pins.

\section{A.7 Communication Hardware}

Wireless communication between TARA and a ground based computer is made possible by using Maxstream's XBee Pro $2.4 \mathrm{GHz}$ radio frequency modules. The XBee module, as shown in Figure 80, has a transmission range of $30 \mathrm{~m}$ for indoor use, which increases up to $90 \mathrm{~m}$ line of sight range for outdoor operation. The XBee module operates on a supply of $3.3 \mathrm{~V}$ and consumes $50 \mathrm{~mA}$ during transmission. The wireless communication speed is 115200 bps, which is enough to send the orientation and position data from TARA to the ground station, as well as to send flight commands to TARA.

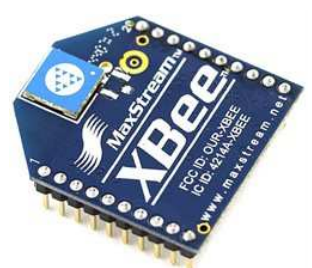

Figure 80: The Xbee Pro wireless transciever module [150].

A 4-channel Futaba radio control system is also connected to TARA for providing manual flight commands. The receiver weighs only $14 \mathrm{~g}$ and is connected to the Arduino controller to send throttle, roll, pitch and yaw commands. 


\section{A.8 Software Implementation}

The autopilot code for both the Axon and Arduino controller boards is written in the $\mathrm{C}++$ programming language. The Arduino board is assigned to perform most of the low-level tasks and runs its programmed function at a rate of $495 \mathrm{~Hz}$. Once powered up, the Arduino board performs basic initializations of the gyroscope, accelerometer, and compass sensors. When the initializations are complete, the Arduino is ready to arm the motors and waits for an arming routine, which is manually provided via the $\mathrm{RF}$ radio controller by a user. This user initiated arming is deliberately implemented as a safety protocol. In this armed state the Arduino starts an attitude control loop which acquires attitude data from the inertial sensors and evaluates appropriate motor speeds for each of the four rotors based on this attitude information. By default, the attitude controller is programmed to keep TARA in a level position, unless a desired attitude is issued as a set command. Any flight commands may either be issued manually using a $\mathrm{RF}$ radio control, or when in autonomous mode, are issued from the Axon and received via $\mathrm{I} 2 \mathrm{C}$ bus. Also, as a safety override, during autonomous mode the user sent RF radio commands for throttle, pitch, roll, and yaw are mixed with the Axon outputs. In autonomous mode, user commands are only issued to apply throttle offsets for takeoff and, if necessary, to correct the lateral position of TARA in order to prevent any collision during experimental flights.

The Axon board is assigned all the high-level tasks to perform as a navigation controller. It initializes the UARTs, timers, PWM, ADC, and I2C functions on startup. The Axon is programmed to acquire data from the GPS and parse it for specific required information; this is done at a $1 \mathrm{~Hz}$ rate. Sonar based range measurements are also made from the sensors mounted on the tip of the four arms of TARA. Furthermore, the Axon maintains a bi-directional wireless XBee connection with a ground 
station computer, which is use for telemetry purposes. All the flight data is transmitted to the computer and logged. Commands are also sent from the computer, which are used for in-flight controller gain tuning. 


\section{Appendix B}

\section{Sonar Based Position Estimation}

Unlike indoor systems which can rely on vision based positioning, for outdoor use in and around the turbulent wind gust conditions generated by urban structures a localization method capable of $\pm 100 \mathrm{~cm}$ error margin is desired. Hence, the autopilot system must be capable of incorporating and using sonar based position data in its control algorithms in order to effectively avoid collisions with urban structures while operating in an outdoor turbulent wind environment. Performing range measurement and position estimation using sonar sensors involves dealing with issues related to employing multiple sonar sensors and various forms of noise (e.g. electrical, vibrations,

and sound) generated by quadrotor itself. A specific sonar triggering scheme and a custom noise cancellation algorithm are developed.

An effective outdoor position control of a UAV depends upon the accuracy of the position estimates, the rate of this position information and the controller's ability to deal with disturbances such as sensor noise as well as environmental effects such as wind gusts. There are several examples found in the literature where a quadrotor's position is controlled with good accuracy. Most of these position control methods depend upon vision based position information which is available in a limited indoor area. Range measuring sensors based on sonar or infrared have been used for position control on few quadrotor platforms. Bouabdallah [31] used a sonar ranging module 
for altitude control in an indoor environment. Roberts et al. [151] used four infrared ranging modules for $\mathrm{X}$ and $\mathrm{Y}$ positioning as well as a sonar ranger for the altitude control of a quadrotor in indoor flight. Chee et al. [152] also employed four infrared range modules for obstacle avoidance and a sonar module for altitude control indoors, with a GPS used for outdoor position control.

\section{B.1 Range Measurement and Position Estimation}

In a typical setup for the position control of a robot in an outdoor environment, GPS is used for position and velocity estimation. In the event of GPS signal loss (number of channels), accelerometers are occassionally used for providing the estimated position and velocity updates. This accelerometer based position information is for temporary use only and becomes unreliable over a longer period of time. The accuracy of available GPS devices is in the range of \pm 3 meters, which is rather large for robots requiring centimeter scale position information. Also, using only GPS is not sufficient for scenarios where the robot may run into an obstacle. Therefore, in order to control the position of TARA flying outdoors in urban areas within close proximity of buildings, such GPS based methodology is not enough. The presence of obstacles such as buildings combined with wind gust conditions can push TARA into objects, a wall for example, resulting in a fatal crash. Sonar based range measurement modules

are therefore used to measure the range from building walls, as well as provide a means to determine the relative position of TARA with respect to its surroundings.

To obtain the position coordinates of TARA, three sonar sensors are used to measure the ranges between TARA and its surrounding environment. Two sonar sensor modules are mounted at the end of both the right and back arms of the quadrotor, as shown in Figure 81, to measure the corresponding right and back ranges. One sonar sensor module is mounted pointing downward at the bottom of the chassis 
to measure the altitude of the UAV. Each sonar sensor can measure a range of $20 \mathrm{~cm}$ to $750 \mathrm{~cm}$ within a centimeter precision at a rate of $10 \mathrm{~Hz}$.

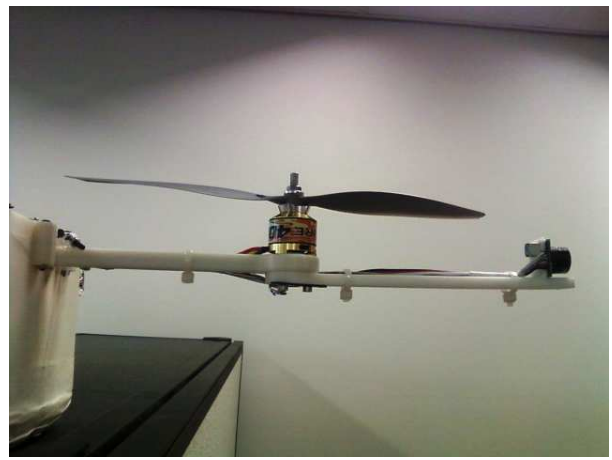

Figure 81: The sonar sensor module mounted at the end of an arm.

Employing multiple sonars within a system introduces concerns with respect to successfully and efficiently allowing for their concurrent use. Simply ranging multiple sensors continuously in a free run mode is not recommended. Such non-synchronous operation with each sonar independently transmitting and receiving at their own speed results in problems with cross-sensor acoustic interference. This interference renders the range readings obtained unreliable.

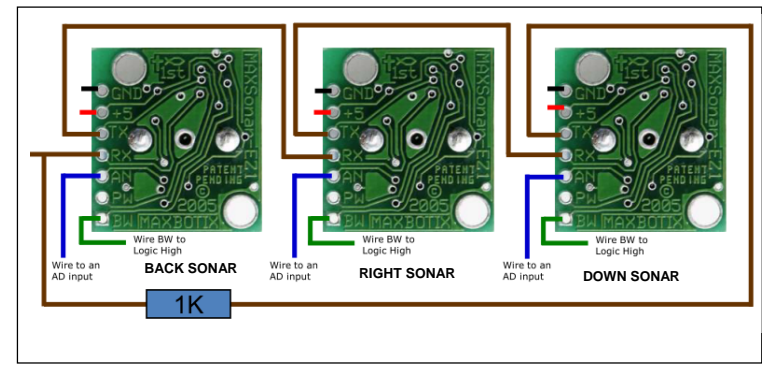

Figure 82: Daisy chaining sonar sensors [153].

Daisy chaining the sonars allows for each respective sensor to be read sequentially. As shown in Figure 82, the transmit output of one sensor connected is to the receive pin of the next sensor in sequence. As a result, each device ranges after the previous device has completed its measurement, thereby eliminating cross-sensor interference. 
However, individually reading each sonar ranger in sequence incurs a time cost which drops the measurement rate down to an undesirable $1 \mathrm{~Hz}$.

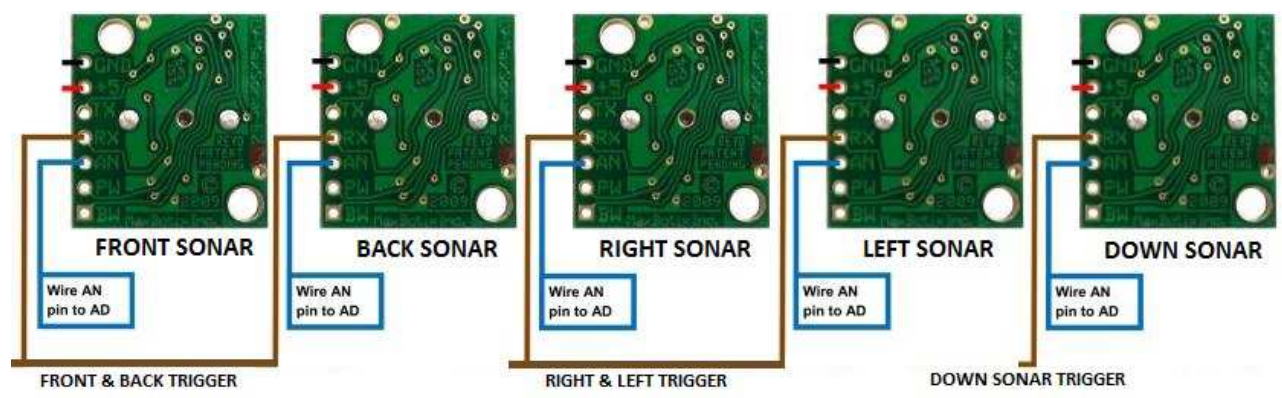

Figure 83: Simultaneous triggering sonar sensors [153].

For the all of the experiments conducted in this research, the receive lines of the Right, Back and Height sensors are connected together such that all three are triggered simultaneously. As depicted in Figure 83, typically five sonars should be used for full 360 degree coverage, however, for the purposes of this study the front and left sonars are not employed. This arrangement is not an ideal case as it allows some cross-sensor interference to occur, however this tradeoff is made in order to achieve a $10 \mathrm{~Hz}$ of position measurement update rate which results in a significant improvement in the control performance.

The quadrotor environment itself also presents inherent issues when operating sonar rangers. Several sources of noise contribute to challenges in obtaining reliable measurements [154]. To begin, the quadrotor's four propellers are an evident source of air turbulence. Mounting the sensors as far away from the propellers as possible is recommended. In addition, the propellers' acoustic noise may be detected by the sonars. Conducted and radiated electric noise, as well as power and grounding also need to be taken into consideration. Finally, the mechanical vibrations of the quadrotor frame contribute to a noisy environment for sonar rangers.

Figure (raw sonar data) illustrates some characteristic raw range data obtained from the sonar measurements. The data in Figure 84(a) shows spurious spikes in value 


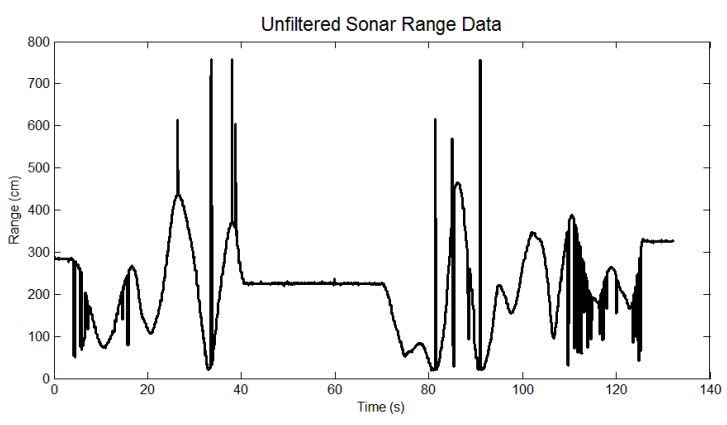

(a)

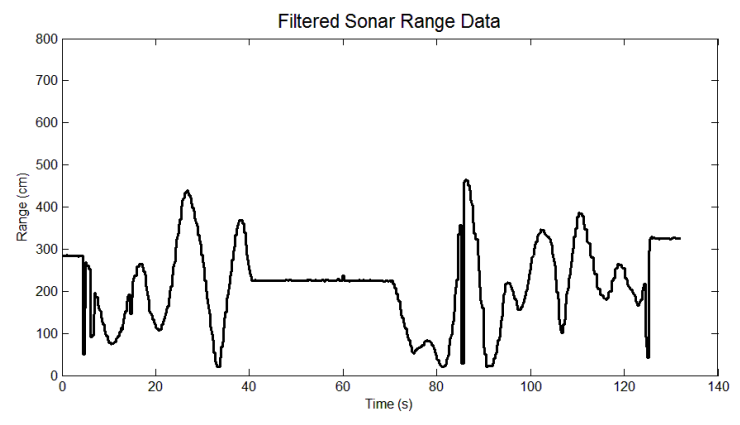

(b)

Figure 84: Thrust plotting of experimental data. (a) Unfiltered and (b) filtered sonar range data.

which are inconsistent with a realistic range value expected within such an abbreviated time period. Proceeding with such unreliable data would provide a substantial impact on the speed calculations and differential gain performance derived from sensor readings. In a control situation, quadrotor performance would be compromised. Thus, several real-time techniques are investigated to filter the sonar data. The performance of both moving average and median filters are not satisfactory in preserving relevant signal characteristics. Hence, a customized sonar filter was developed. If the current range value obtained by a sonar sensor is found to be $\pm 80 \mathrm{~cm}$ away from its previous range value within a time interval of 0.1 seconds, it is assumed that this is not a valid reading due to the fact that TARA is programmed to fly with speeds less than $8 \mathrm{~m} / \mathrm{s}$. This invalid reading needs to be replaced with meaningful data. A linear estimation is performed based on the last 2 previous range values. The direction of the slope is based on TARA's velocity (from accelerometers). The filtered sonar range as shown in Figure 84(b) is then limited between 20 and $600 \mathrm{~cm}$. 


\section{B.2 Heading Independent Lateral Position Con- trol Scheme}

For both indoor and outdoor experimental setups, the control performance is dependent on the stability of the yaw angle. It is desired that the heading controller be able to maintain the heading during the test flight. Disturbances to the heading control originate from many sources, including the $\mathrm{X}, \mathrm{Y}$ and $\mathrm{Z}$ position controller. These disturbances cause the heading angle to deviate and fluctuate about the desired

value. A position control scheme is developed in order to incorporate the effects of any heading deflections on the range estimates and move along the vector pointing towards the desired position. Instead of implementing two controllers for the $\mathrm{X}$ and Y-axes independently, a single PD position controller can be used which takes the magnitude of the vector from TARA's current position to the desired position as the error value.

TARA's absolute position is determined with respect to the ordinal coordinate system (North, East, South, and West). As depicted in Figure 85, the range measurements $R_{R}$ and $R_{B}$, referring to the right range and the back range respectively, are obtained with reference to TARA's body position. Thus the error becomes:

$$
\begin{gathered}
|\vec{E}|=\sqrt{\left(E_{X}\right)^{2}+\left(E_{Y}\right)^{2}} \\
\gamma_{E}=\tan ^{-1}\left(\frac{E_{Y}}{E_{X}}\right)
\end{gathered}
$$

From Figure 85 it can be seen that $E_{X}$ and $E_{Y}$ are defined as:

$$
E_{X}=-R_{B} \cos \left(\gamma_{\psi}\right)-X_{r e f}
$$




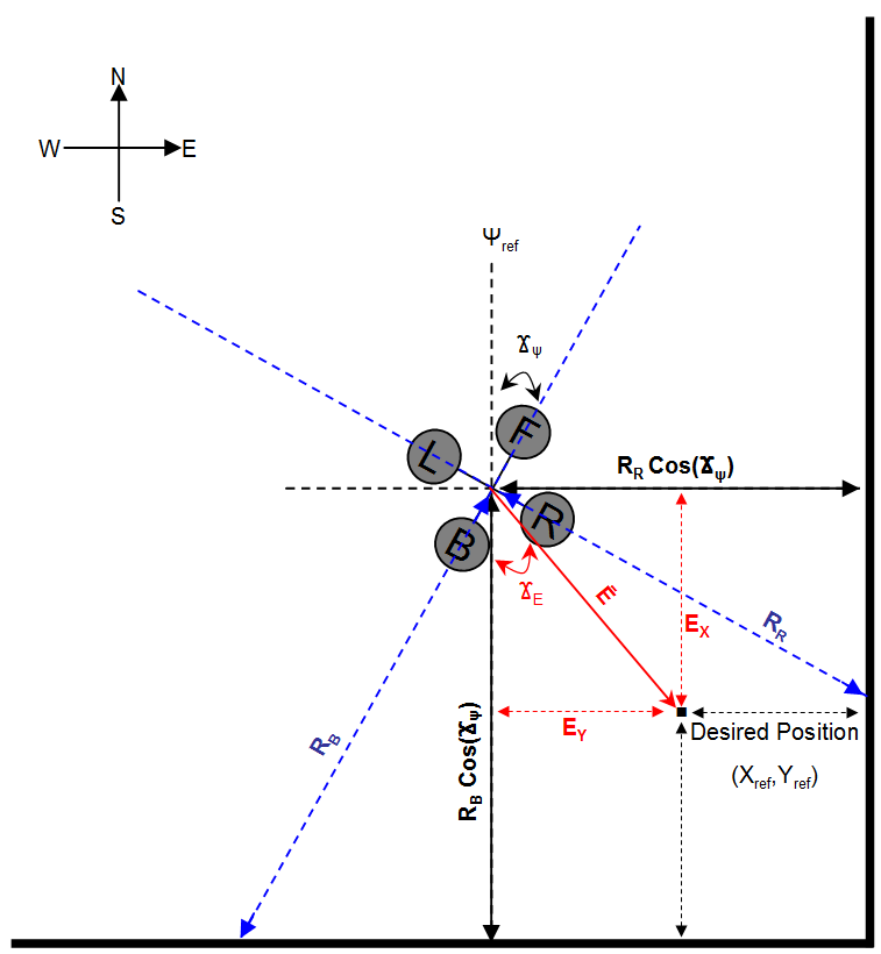

Figure 85: Updated position control scheme.

$$
E_{Y}=R_{R} \cos \left(\gamma_{\psi}\right)-Y_{\text {ref }}
$$

where the heading error with respect to the desired value is:

$$
\gamma_{\psi}=\left(\psi-\psi_{\text {ref }}\right)
$$

Using these results allows the magnitude and angle of the error vector to be expressed as:

$$
\begin{gathered}
|\vec{E}|=\sqrt{\left(-R_{B} \cos \left(\gamma_{\psi}\right)-X_{r e f}\right)^{2}+\left(R_{R} \cos \left(\gamma_{\psi}\right)-Y_{r e f}\right)^{2}} \\
\gamma_{E}=\tan ^{-1}\left(\frac{-R_{B} \cos \left(\gamma_{\psi}\right)-Y_{r e f}}{R_{R} \cos \left(\gamma_{\psi}\right)-X_{r e f}}\right)
\end{gathered}
$$


while the combined XY position control law is then written:

$$
U_{X Y}=K_{P_{X Y}}|\vec{E}|+K_{D_{X Y}} \dot{\vec{E}}
$$

with $K_{P_{X Y}}=0.2$ and $K_{D_{X Y}}=-4.0$. The control law for the altitude remains uncoupled from the XY position control and is unchanged.

Based on the updated position control, the following pitch and roll axis control commands are generated:

$$
\begin{gathered}
U_{\text {pitch }}=U_{X Y} \cos \left(\gamma_{E}-\gamma_{\psi}\right) \\
U_{\text {roll }}=U_{X Y} \sin \left(\gamma_{E}+\gamma_{\psi}\right)
\end{gathered}
$$




\section{Appendix $\mathrm{C}$}

\section{Position Control Simulations}

Supplementary results of position control are provided in this Appendix. The simulations conducted in no wind cases are detailed in first section. The second section contains position control plots in wind cases where a full 12 minute flight time for the waypoint scheme is shown. The third section includes the experimental outdoor flight plots including the manual radio control command data during the autonomous position hold.

\section{C.1 Position Control Simulations without Wind}

Each of the four presented controllers is tested in the absence of wind for the six defined single building simulation test cases. The simulation results of one minute position hold at point-B are presented here to show that the performance of each control technique meets the desired good position hold criteria. Also, the results serve as a reference to observe the impact of wind disturbances on the control performance. 
Table 24: No wind control performance summary of position hold at point-B.

\begin{tabular}{|l|l|c|c|c|c|}
\cline { 3 - 6 } \multicolumn{2}{c|}{} & PID & IB & Fuzzy & AIB \\
\hline \multirow{3}{*}{ X-axis } & Max. Error $(\mathrm{m})$ & 0.15 & 0.74 & 0.41 & 0.81 \\
\cline { 2 - 6 } & AOAD $(\mathrm{m})$ & 0.06 & 0.28 & 0.15 & 0.25 \\
\hline \multirow{2}{*}{ Y-axis } & Max. Error $(\mathrm{m})$ & 0.10 & 0.38 & 0.18 & 0.57 \\
\cline { 2 - 6 } & AOAD $(\mathrm{m})$ & 0.03 & 0.16 & 0.05 & 0.18 \\
\hline \multirow{2}{*}{ Z-axis } & Max. Error $(\mathrm{m})$ & 0.02 & 0.02 & 0.12 & 0.02 \\
\cline { 2 - 6 } & AOAD $(\mathrm{m})$ & 0.01 & 0.01 & 0.10 & 0.01 \\
\hline
\end{tabular}

\section{C.1.1 Sample Position Hold at Point-B}

\section{C.2 In wind Position Control Simulation Plots}

Provided here are the plots showing the full 12 minute simulation flight test used for evaluating the position control performances of the control techniques. The presented results also serves in demonstrating the trajectory tracking performance of the PID, AIB, and the proposed hybrid control techniques.

\section{C.3 Experimental Outdoor Flight Plots}

Experimental outdoor flight using the PID control for the position hold of TARA. Illustrated plots include position error, radio control command, and the wall position as reference.

Experimental outdoor flight data plots using the hybrid control for the position hold of TARA. 


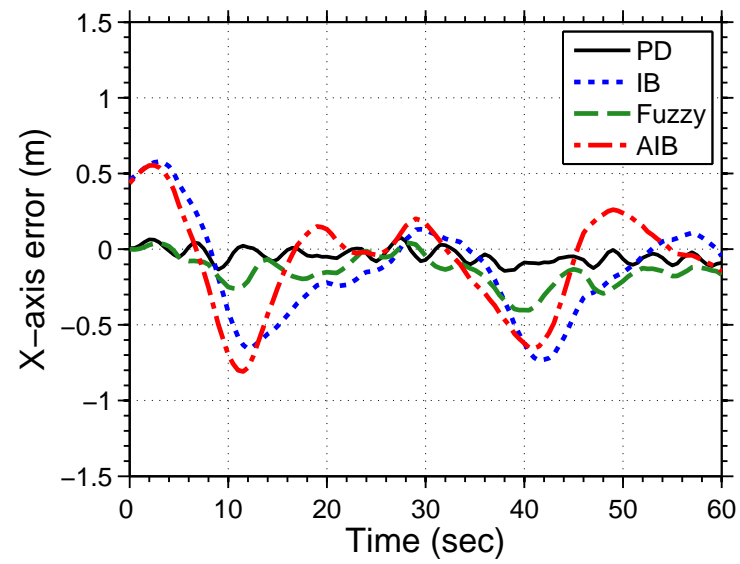

(a)

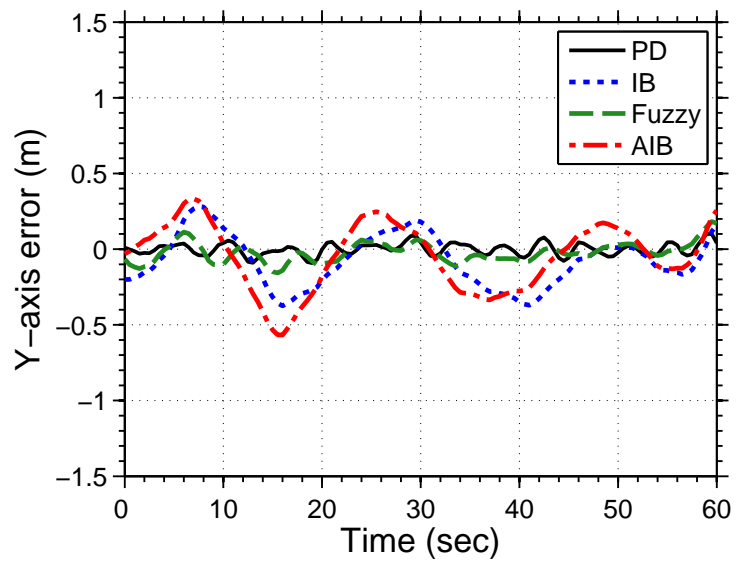

(b)

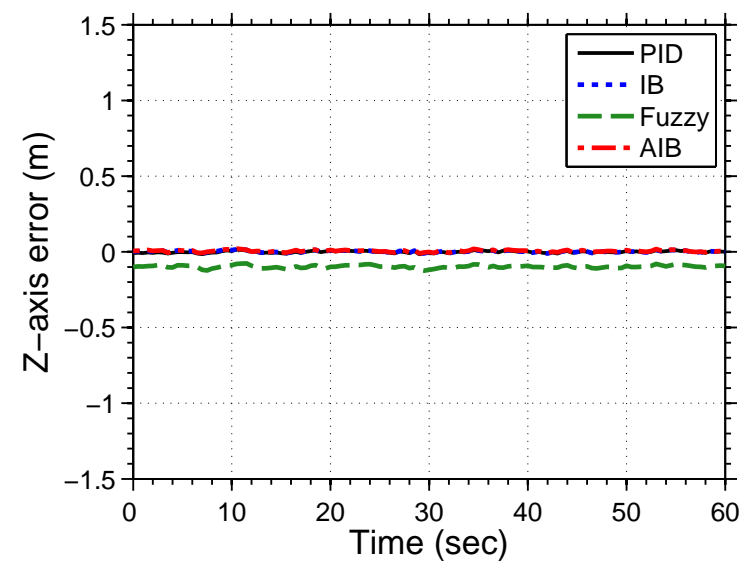

(c)

Figure 86: No wind position hold for passing vortex (Point-B). (a) X-axis; (b) Y-axis; and (c) Z-axis.

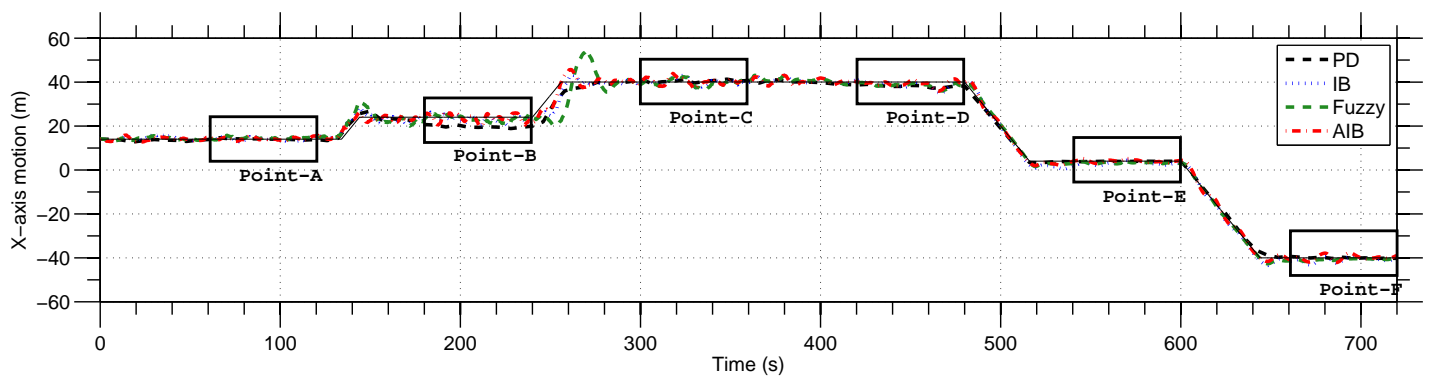

Figure 87: Full flight test plot for $\mathrm{X}$-axis. 


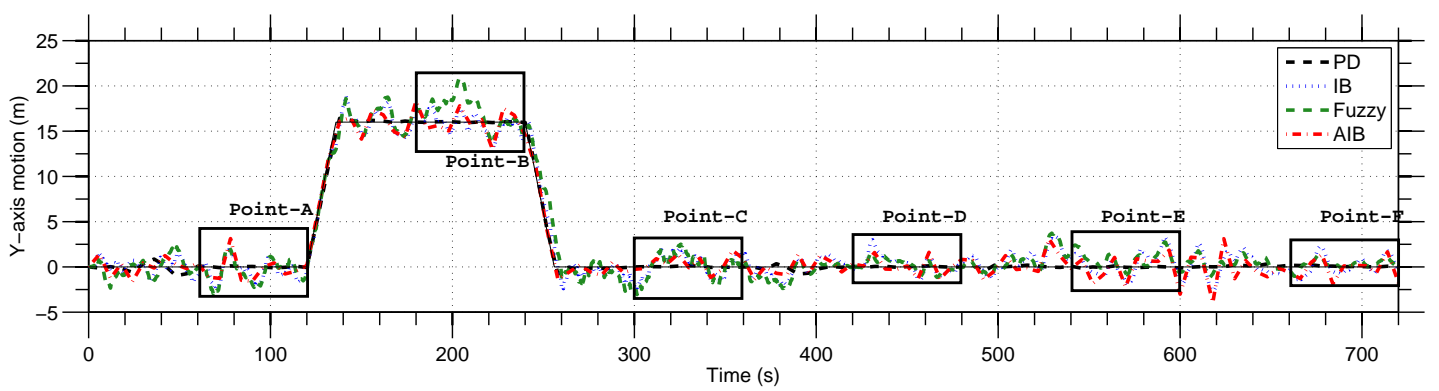

Figure 88: Full flight test plot for Y-axis.

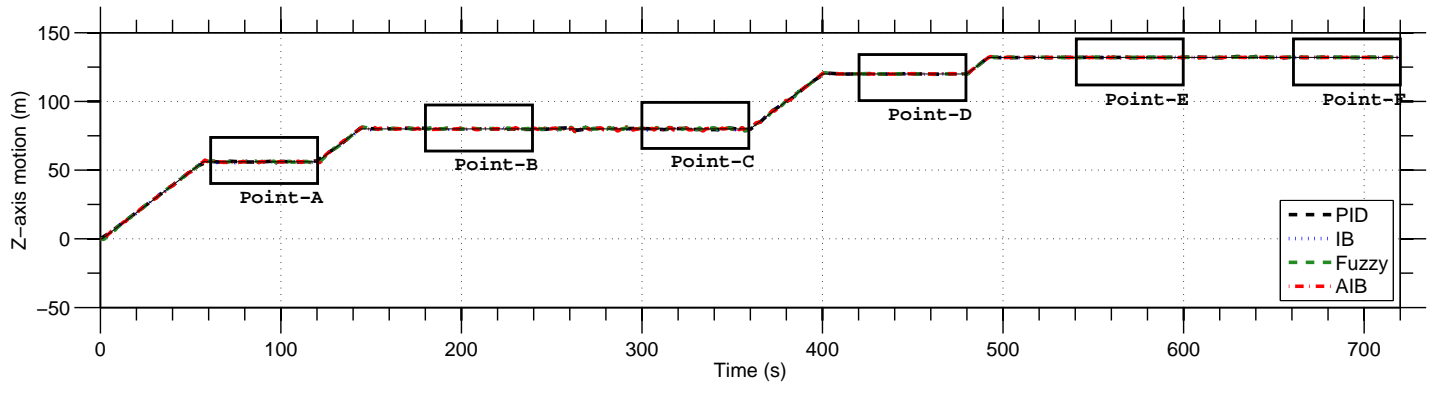

Figure 89: Full flight test plot for Z-axis. 


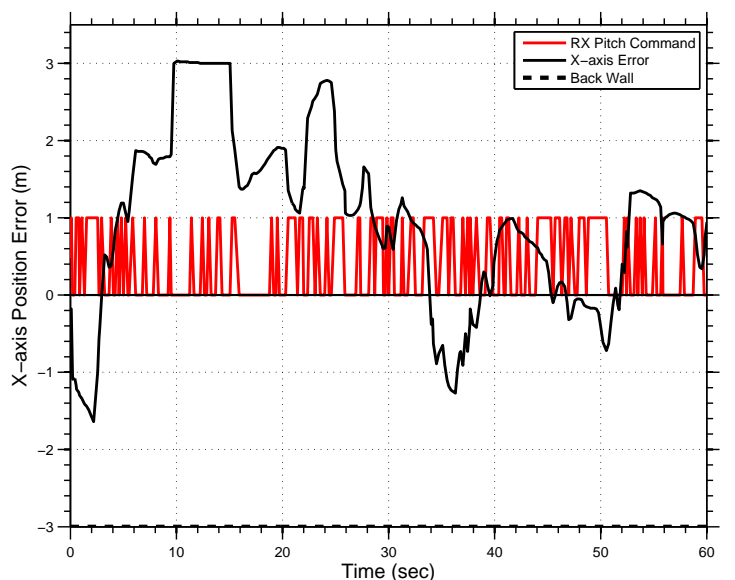

(a)

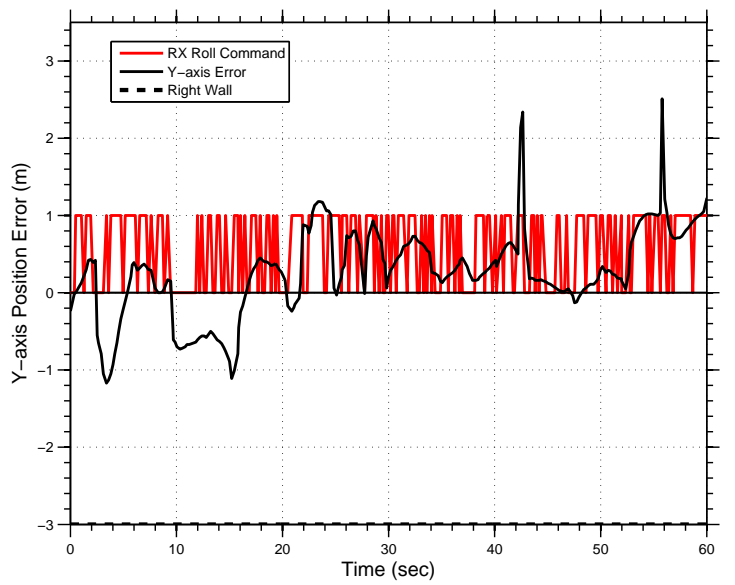

(c)

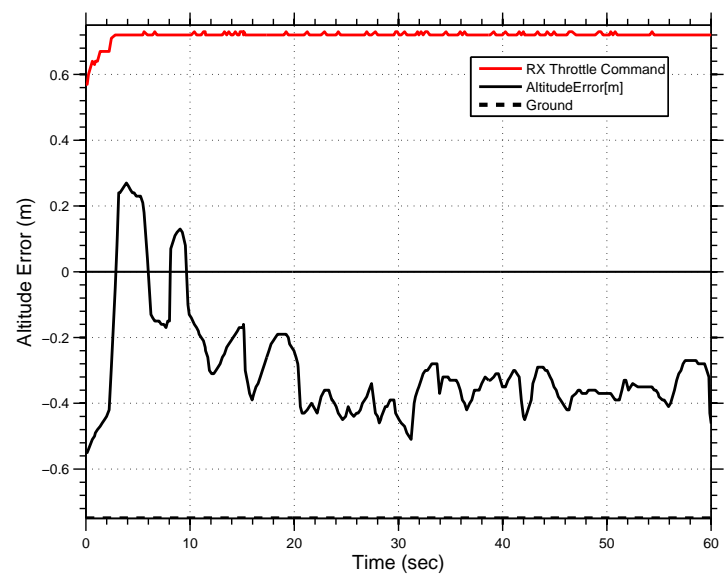

(e)

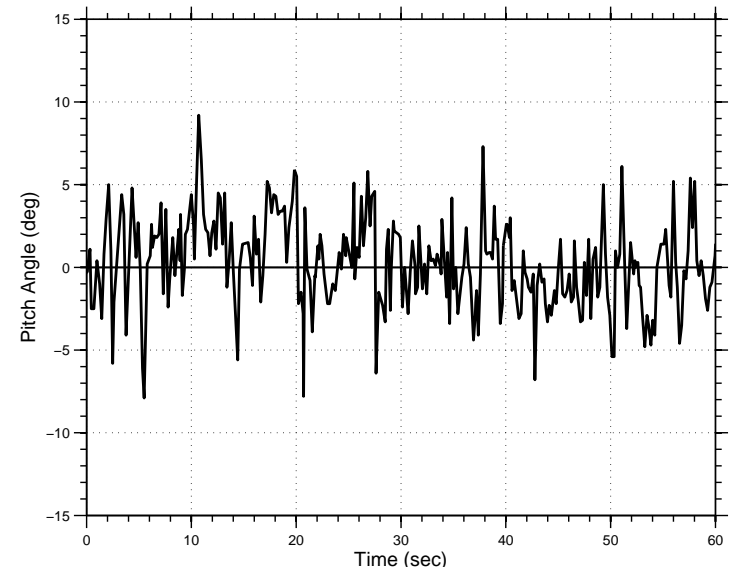

(b)

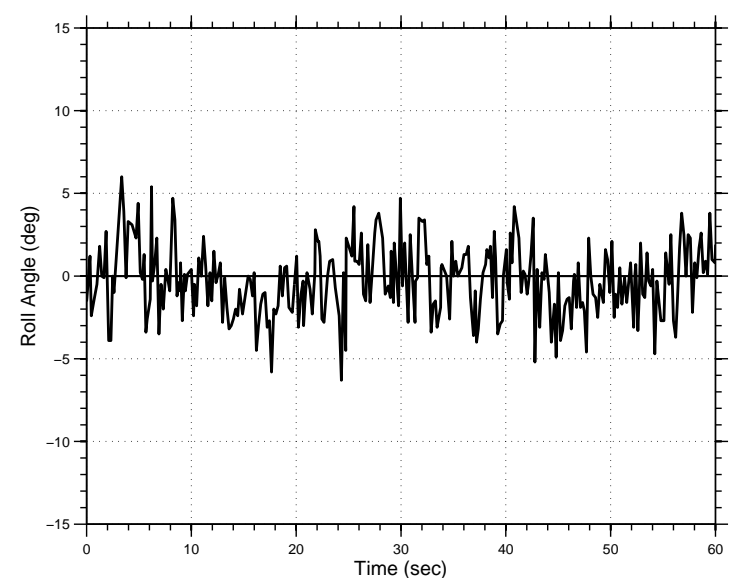

(d)

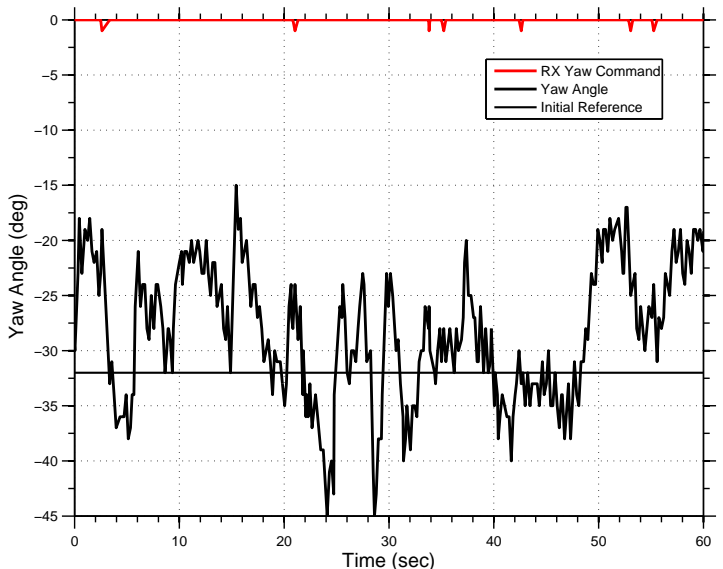

(f)

Figure 90: PID outdoor position control plots. (a) X-axis; (b) Pitch angle; (c) Y-axis; (d) Roll angle; (e) Z-axis; and (f) Yaw angle. 


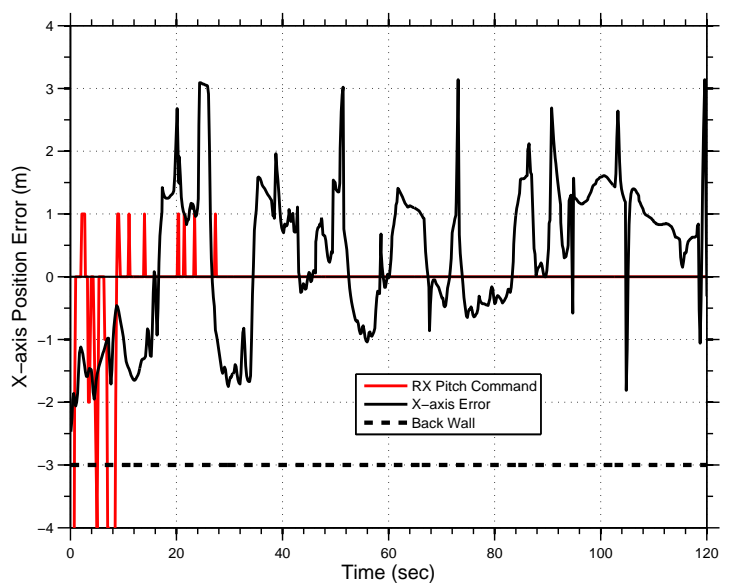

(a)

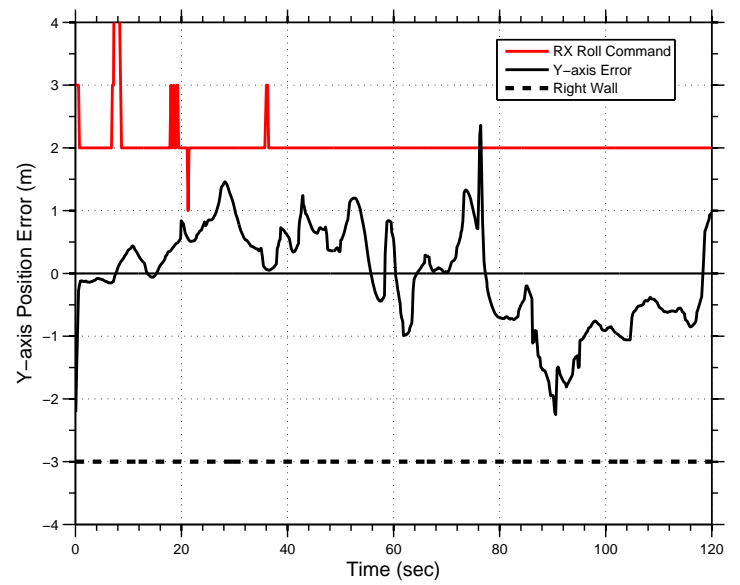

(c)

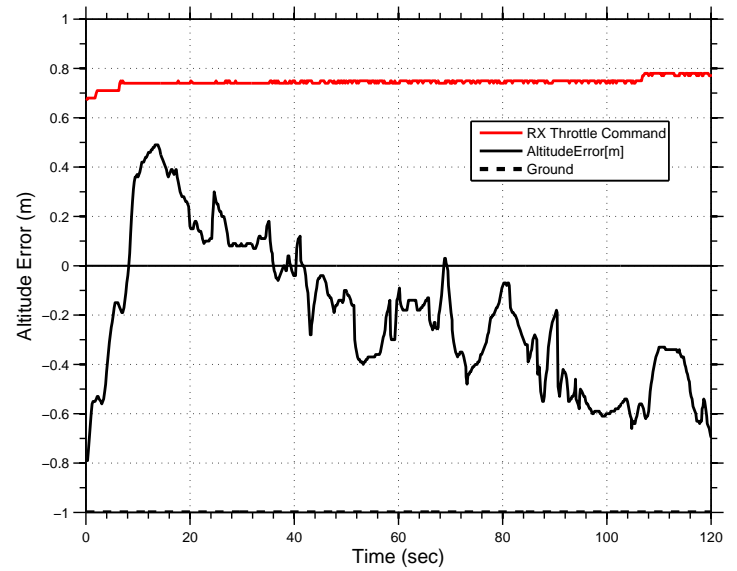

(e)

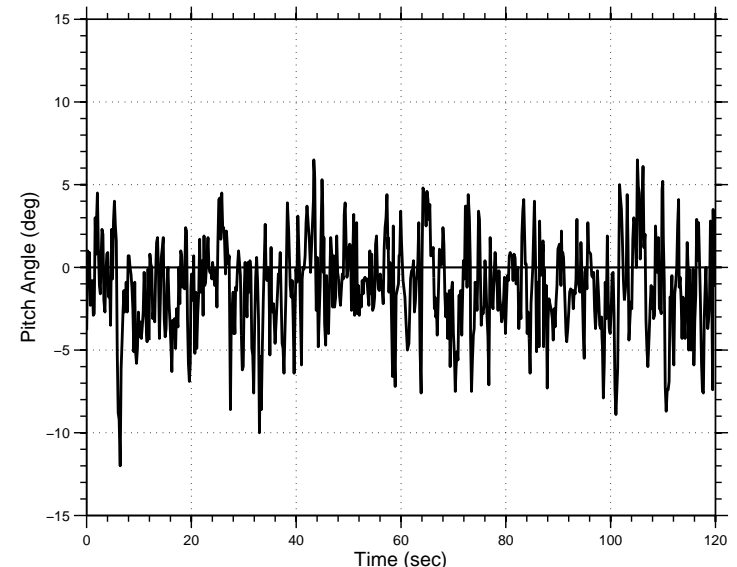

(b)

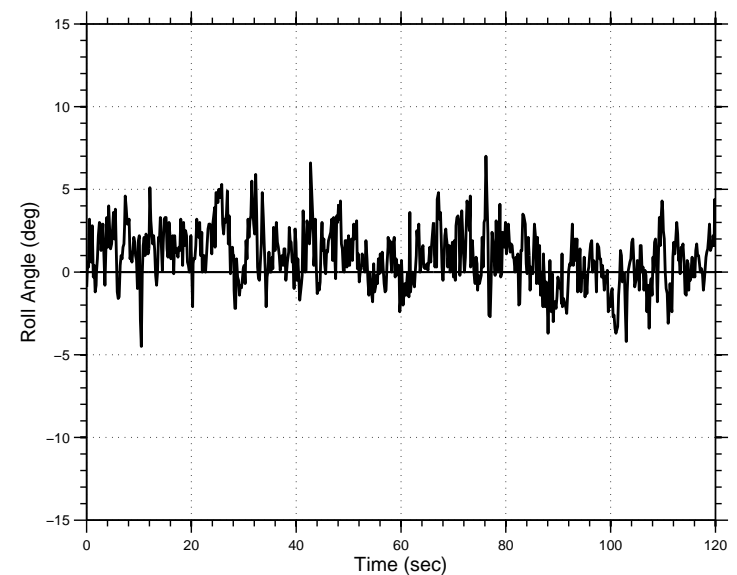

(d)

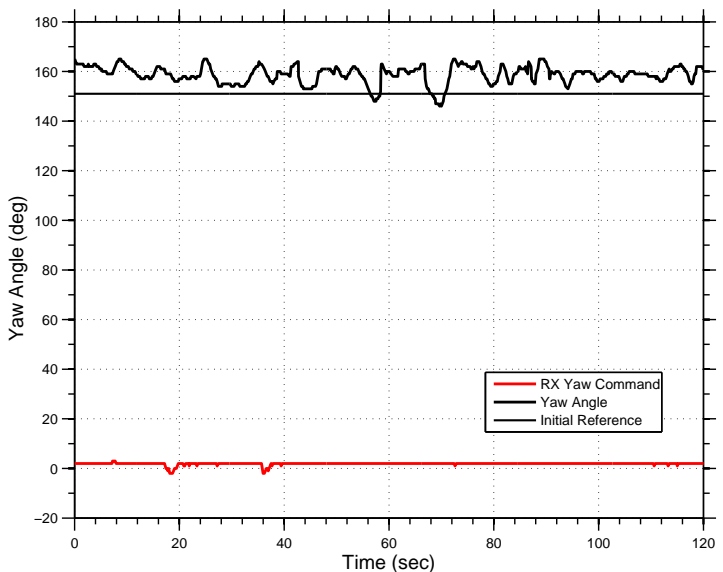

(f)

Figure 91: Hybrid outdoor position control plots. (a) X-axis; (b) Pitch angle; (c) Y-axis; (d) Roll angle; (e) Z-axis; and (f) Yaw angle. 